\title{
The Influence of Substitution on Thiol-Induced Oxanorbornadiene
}

\section{Fragmentation}

\section{Table of Contents}

Lucrezia De Pascalis ${ }^{\dagger}$, Mei-Kwan Yau ${ }^{\dagger}$, Dennis Svatunek, Zhuoting Tan, Srinivas Tekkam, K. N. Houk, and M.G. Finn*

\section{Supporting Information}

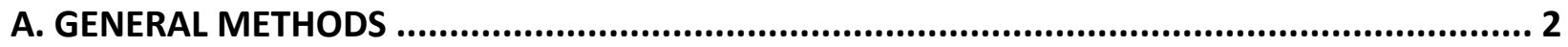

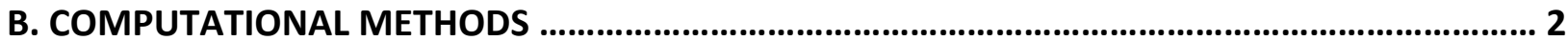

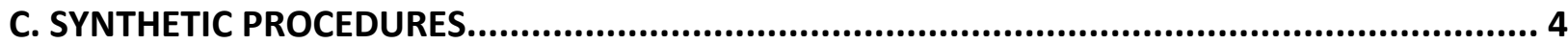

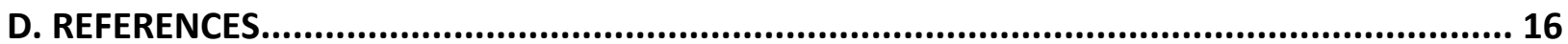

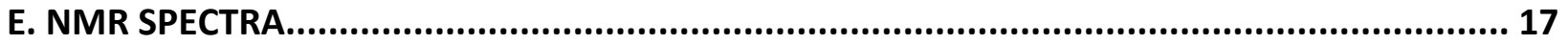




\section{A. GENERAL METHODS}

Unless otherwise noted, all reactions were carried out in oven-dried glassware under an atmosphere of dry argon. Dichloromethane, toluene, and tetrahydrofuran were dried by passage through activated alumina (MBraun solvent purification system). All commercially available reagents were purchased from Acros Organics, Alfa Aesar, Ark Pharm, Sigma Aldrich, ChemImpex, Combi-Blocks, Fisher, Fluka, Frontier Scientific, J\&W Pharmlab, Oakwood Chemical, OxChem, Strem Chemicals, or $\mathrm{TCl}$ Chemicals and were used as received. All deuterated solvents were purchased from Cambridge Isotope Laboratories.

NMR spectra were obtained on Brüker AMX-400, DRX-500 or AMX-700 instruments. Chemical shifts are reported relative to the central line of residual solvent chloroform $\quad$ (7.26

ppm, ${ }^{1} \mathrm{H} ; \quad 77.00 \mathrm{ppm},{ }^{13} \mathrm{C}$ ) and methanol $\left(3.31 \quad \mathrm{ppm},{ }^{1} \mathrm{H} ; \quad 49.00 \mathrm{ppm},{ }^{13} \mathrm{C}\right)$. ${ }^{13} \mathrm{C}$ NMR were obtained with ${ }^{1} \mathrm{H}$ decoupling. High-resolution mass spectrometry was performed on an Agilent 6230 ESI-TOF LC/MS instrument (G6230B) operating at $4 \mathrm{GHz}$ with internal reference. LC was performed on an Agilent 1260 HPLC with a mobile phase gradient from 2$100 \%$ acetonitrile/ water containing $0.1 \%$ formic acid on a Zorbax Extend-C18 Rapid Resolution HT $(2.1 \times 50 \mathrm{~mm}, 1.8 \mu \mathrm{m})$.

Analytical thin layer chromatography (TLC) was performed using MilliporeSigma aluminum plates coated with $0.25 \mathrm{~mm}$ silica gel containing PF 254 indicator, and the compounds were visualized with UV light and potassium permanganate stain. Flash chromatography was carried out with Silicycle SilicaFlash. F60 (particle size 40-63 $\mu \mathrm{m}$ ) silica gel or it was performed on the Biotage ${ }^{\circledR}$ Automated Liquid Chromatography System Isolera One ${ }^{\circledR}$ using Biotage $®$ SNAP KP. Sil $10-25 g$ or ZIP $5 g$ Si silica gel cartridges.

\section{B. COMPUTATIONAL PROCEDURES}

Theoretical calculations were performed using the software package Gaussian 16, Revision A.03. ${ }^{1}$ Geometry optimizations and frequency analyses were performed using the M06-2X density functional and the $6-311+G(d, p)$ basis set in chloroform (SMD) in combination with Grimme D3 empirical dispersion correction ${ }^{3}$ without Becke-Johnson dampening as proposed by Grimme and coworkers. ${ }^{4}$ Quasi-harmonic correction was performed by using the "Goodvibes" Python script by Robert Paton. ${ }^{5}$ Single imaginary frequencies corresponding to the desired reaction coordinates were obtained only in the case of transition state calculations. No imaginary frequencies were obtained for all other structures. Table S1 shows the calculated energies. Coordinates are provided as *.xyz files. 
Table S1. M06-2X-D3/6-311+G(d,p)(chloroform) calculated energies.

\begin{tabular}{|c|c|c|c|c|}
\hline Structure & $\Delta \mathrm{E}$ (hartree) & ZPE (hartree) & $\Delta \mathrm{H}_{298}$ (hartree) & $\Delta \mathrm{G}_{298}$ (hartree) \\
\hline $2 a$ & -1602.963095 & 0.392363 & -1602.543485 & -1602.623120 \\
\hline $2 b$ & -1622.820640 & 0.379620 & -1622.414074 & -1622.493201 \\
\hline $2 c$ & -1853.844844 & 0.461888 & -1853.351638 & -1853.439182 \\
\hline 2d & -1622.822577 & 0.380113 & -1622.416029 & -1622.493737 \\
\hline $2 e$ & -1622.819747 & 0.379805 & -1622.412824 & -1622.492296 \\
\hline $2 f$ & -1814.532883 & 0.433625 & -1814.069496 & -1814.154069 \\
\hline $2 g$ & -1929.048906 & 0.466213 & -1928.550360 & -1928.639830 \\
\hline $2 h$ & -2019.025407 & 0.435563 & -2018.557060 & -2018.647624 \\
\hline $2 \mathbf{i}$ & -2151.586162 & 0.437702 & -2151.114622 & -2151.207206 \\
\hline $2 \mathrm{j}$ & -1876.665032 & 0.382074 & -1876.253504 & -1876.337779 \\
\hline $2 k$ & -1668.655117 & 0.325608 & -1668.304231 & -1668.379856 \\
\hline 2 & -1530.105294 & 0.304595 & -1529.777459 & -1529.848937 \\
\hline $2 m$ & -2296.898678 & 0.424630 & -2296.439060 & -2296.533461 \\
\hline $2 n$ & -2222.980884 & 0.443254 & -2222.502689 & -2222.597204 \\
\hline 20 & -2544.000408 & 0.460204 & -2543.501942 & -2543.602599 \\
\hline $2 p$ & -1547.551412 & 0.375453 & -1547.150797 & -1547.226103 \\
\hline $2 q$ & -1622.779169 & 0.380168 & -1622.373020 & -1622.449582 \\
\hline 8 & -1432.826713 & 0.326289 & -1432.477795 & -1432.548116 \\
\hline 9 & -1432.825040 & 470 & -1432.476212 & -1432.545906 \\
\hline 10 & -1432.830606 & 0.326991 & -1432.481239 & -1432.551048 \\
\hline 11 & -1432.832123 & 0.326811 & -1432.482776 & -1432.552815 \\
\hline 12 & -1538.854375 & 0.250734 & -1538.582366 & -1538.650356 \\
\hline 13 & -1538.854524 & 0.250810 & -1538.582401 & -1538.650275 \\
\hline 14 & -1538.852944 & 0.249724 & -1538.581639 & -1538.6 \\
\hline 15 & 1538.846246 & 0.249976 & -1538.574972 & -1538.642681 \\
\hline 16 & -1201.800981 & 0.245789 & -1201.537479 & -1201.598108 \\
\hline TS2a & -1602.913440 & 0.388204 & -1602.497204 & -1602.578359 \\
\hline TS2b & -1622.774043 & 0.375706 & -1622.370697 & -1622.451426 \\
\hline TS2c & -1853.793691 & 0.457853 & -1853.304004 & -1853.392656 \\
\hline TS2d & -1622.777333 & 908 & -1622.37 & -1622.4 \\
\hline TS2e & -1622.774531 & 0.375793 & -1622.371108 & -1622.451338 \\
\hline TS2f & -1814.493067 & 0.429501 & -1814.032986 & -1814.118950 \\
\hline TS2g & -1929.010091 & 0.462885 & -1928.514287 & -1928.604681 \\
\hline TS2h & -2018.984392 & 0.432268 & -2018.518977 & -2018.610195 \\
\hline TS2i & -2151.545640 & & -2151.077033 & -2151.169835 \\
\hline TS2j & -1876.620683 & 735 & -1876.211541 & -1876.297687 \\
\hline TS2k & -1668.611537 & 0.323378 & -1668.262640 & -1668.339018 \\
\hline TS2I & -1530.055689 & 0.301242 & -1529.730712 & -1529.803383 \\
\hline TS2m & -2296.842518 & 0.421887 & -2296.385442 & -2296.480073 \\
\hline TS2n & -2222.944787 & 0.439953 & -2222.469401 & -2222.565009 \\
\hline TS20 & -2543.963486 & 0.456884 & -2543.467765 & -2543.569700 \\
\hline TS2p & -1547.510662 & 0.372073 & -1547.112569 & -1547.189819 \\
\hline TS2q & -1622.737632 & 0.376875 & -1622.334064 & -1622.412208 \\
\hline TS8 & -1432.786666 & 0.322158 & -1432.441129 & -1432.512805 \\
\hline TS9 & -1432.784174 & 0.322949 & -1432.438270 & -1432.509373 \\
\hline TS10 & -1432.785633 & 0.323236 & -1432.439447 & -1432.510541 \\
\hline TS11 & 1432.786964 & 0.323395 & -1432.44059 & -1432.511474 \\
\hline TS12 & -1538.806516 & 0.246695 & -1538.537901 & -1538.607410 \\
\hline TS13 & -1538.807307 & 0.246580 & -1538.538744 & -1538.608139 \\
\hline TS14 & -1538.802382 & 0.246087 & -1538.534093 & -1538.603808 \\
\hline TS15 & -1538.797052 & 0.246404 & -1538.528742 & -1538.598050 \\
\hline TS16 & -1201.756382 & 0.241444 & -1201.496401 & -1201.558938 \\
\hline
\end{tabular}




\section{SYNTHETIC PROCEDURES}

Acetate esters - general procedure: The furan (1 equiv) was dissolved in $\mathrm{CH}_{2} \mathrm{Cl}_{2}$. Acetic anhydride (3 equiv) and trimethylamine (5 equiv) were added. The mixture was

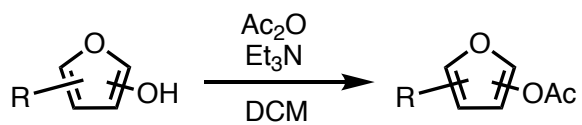
stirred at room temperature overnight. Saturated $\mathrm{NH}_{4} \mathrm{Cl}$ was added and the organic layer was extracted with $\mathrm{CH}_{2} \mathrm{Cl}_{2}$ three times. The combined organic layer was washed with brine, dried over sodium sulfate and the solvent was removed under vacuum. The residue obtained was purified by silica gel chromatography ( $20 \%$ ethyl acetate in hexanes).

\section{(5-methylfuran-3-yl)methyl acetate, S1}

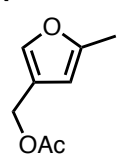

Colorless oil; yield 71\% (112 mg); ${ }^{1} \mathrm{H}$ NMR (500 MHz, $\left.\mathrm{CDCl}_{3}\right): \delta 7.31(\mathrm{~s}, 1 \mathrm{H})$, $6.02(\mathrm{~s}, 1 \mathrm{H}), 4.91(\mathrm{~s}, 2 \mathrm{H}), 2.27(\mathrm{~d}, \mathrm{~J}=0.9 \mathrm{~Hz}, 3 \mathrm{H}), 2.06(\mathrm{~s}, 3 \mathrm{H}) .{ }^{13} \mathrm{C}$ NMR $(125$ $\left.\mathrm{MHz}_{2} \mathrm{CDCl}_{3}\right): \delta 170.9,153.2,139.7,121.1,106.5,58.0,21.0,13.4$. HRMS (ESI-TOF) $\mathrm{m} / \mathrm{z}:[\mathrm{M}+\mathrm{Na}]^{+}$Calcd for $\mathrm{C}_{8} \mathrm{H}_{10} \mathrm{O}_{3} \mathrm{Na}^{+}$calc 177.0528; Found 177.0534.

\section{(5-phenylfuran-3-yl)methyl acetate, S2}

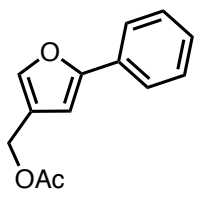

White solid; yield 92\% (231 mg); ${ }^{1} \mathrm{H}$ NMR(500 MHz, $\left.\mathrm{CDCl}_{3}\right): \delta 7.66-7.64(\mathrm{~m}$, $2 \mathrm{H}), 7.50(\mathrm{~s}, 1 \mathrm{H}), 7.40-7.37(\mathrm{~m}, 2 \mathrm{H}), 7.29-7.27(\mathrm{~m}, 1 \mathrm{H}), 6.69(\mathrm{~s}, 1 \mathrm{H}), 5.01(\mathrm{~s}$, $2 \mathrm{H}), 2.10(\mathrm{~s}, 3 \mathrm{H}) .{ }^{13} \mathrm{C}$ NMR $\left(176 \mathrm{MHz}, \mathrm{CDCl}_{3}\right): \delta 170.9,154.8,140.9,130.5$, 128.7, 127.6, 123.8, 122.4, 105.8, 57.8, 21.0. HRMS (ESI-TOF) m/z: $\left[\mathrm{M}+\mathrm{Na}^{+}\right.$ Calcd for $\mathrm{C}_{13} \mathrm{H}_{12} \mathrm{O}_{3} \mathrm{Na}^{+}$239.0684; Found 239.0685.

\section{(5-(4-methoxyphenyl)furan-3-yl)methyl acetate, S3}

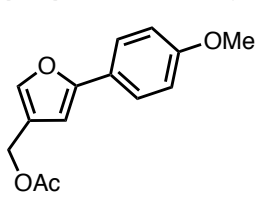

Pink solid; yield 88\% (170 mg); ${ }^{1} \mathrm{H}$ NMR $\left(500 \mathrm{MHz}, \mathrm{CDCl}_{3}\right): \delta 7.59-7.56(\mathrm{dt}, \mathrm{J}=$ 2.8, $9.6 \mathrm{~Hz}, 2 \mathrm{H}), 7.45(\mathrm{~d}, \mathrm{~J}=0.7 \mathrm{~Hz}, 1 \mathrm{H}), 6.92-6.89(\mathrm{dt}, \mathrm{J}=2.8,9.7 \mathrm{~Hz}, 2 \mathrm{H})$, 6.55- $6.54(\mathrm{~d}, \mathrm{~J}=0.5 \mathrm{~Hz}, 1 \mathrm{H}), 4.99(\mathrm{~s}, 2 \mathrm{H}), 3.81(\mathrm{~s}, 3 \mathrm{H}), 2.09(\mathrm{~s}, 3 \mathrm{H}) .{ }^{13} \mathrm{C}$ NMR $\left(125 \mathrm{MHz}, \mathrm{CDCl}_{3}\right): \delta 170.8,159.1,154.7,140.2,125.2,123.4,122.2,114.0$, 104.1, 57.8, 55.1, 20.9. HRMS (ESI-TOF) $\mathrm{m} / \mathrm{z}:\left[\mathrm{M}+\mathrm{Na}^{+}\right.$Calcd for $\mathrm{C}_{14} \mathrm{H}_{14} \mathrm{O}_{4} \mathrm{Na}^{+}$269.0790; Found 269.0788.

\section{(5-(4-nitrophenyl)furan-3-yl)methyl acetate, S4}

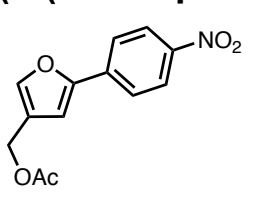

Yellow solid; yield 73\% (25 mg); ${ }^{1} \mathrm{H}$ NMR (500 MHz, $\left.\mathrm{CDCl}_{3}\right): \delta 8.26-8.23(\mathrm{dt}$, $\mathrm{J}=2.3,9.3 \mathrm{~Hz}, 2 \mathrm{H}), 7.79-7.76(\mathrm{dt}, \mathrm{J}=2.3,9.3 \mathrm{~Hz}, 2 \mathrm{H}), 7.60(\mathrm{~d}, \mathrm{~J}=0.7 \mathrm{~Hz}, 1 \mathrm{H})$, $6.91(\mathrm{~s}, 1 \mathrm{H}), 5.01(\mathrm{~s}, 2 \mathrm{H}), 2.10(\mathrm{~s}, 3 \mathrm{H}) .{ }^{13} \mathrm{C} \mathrm{NMR}\left(125 \mathrm{MHz}, \mathrm{CDCl}_{3}\right): \delta 170.8$, 152.4, 146.6, 142.9, 136.0, 124.3, 124.0, 123.2, 109.7, 57.4, 20.9. HRMS (ESITOF) $\mathrm{m} / \mathrm{z}$ : $[\mathrm{M}+\mathrm{Na}]^{+}$Calcd for $\mathrm{C}_{14} \mathrm{H}_{14} \mathrm{O}_{4} \mathrm{Na}^{+} 284.0535$; Found 284.0542 .

\section{(5-(4-(trifluoromethyl)phenyl)furan-3-yl)methyl acetate, S5}

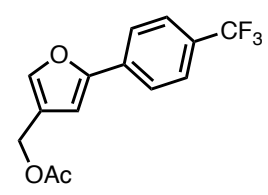

White solid; yield $87 \%(18 \mathrm{mg}) ;{ }^{1} \mathrm{H}$ NMR $\left(500 \mathrm{MHz}, \mathrm{CDCl}_{3}\right): \delta 7.75-7.74$ (d, J= $8.1 \mathrm{~Hz}, 2 \mathrm{H}$ ), 7.64- 7.62 (d, J= $8.2 \mathrm{~Hz}, 2 \mathrm{H}), 7.55$ (s, 1H), 6.80 (s, 1H), 5.01 (s, $2 \mathrm{H}), 2.10(\mathrm{~s}, 3 \mathrm{H}) .{ }^{13} \mathrm{C}$ NMR $\left(125 \mathrm{MHz}, \mathrm{CDCl}_{3}\right): \delta 170.9,153.2,141.9,133.6$, 125.7- 125.7, 123.8, 122.8, 107.8, 57.6, 21.0. HRMS (ESI-TOF) m/z: [M + H] ${ }^{+}$ Calcd for $\mathrm{C}_{14} \mathrm{H}_{11} \mathrm{~F}_{3} \mathrm{O}_{3} \mathrm{H}^{+}$calc 285.0739; found 285.0734.

\section{(4-methylfuran-3-yl)methyl acetate, S6}

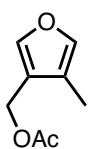

Colorless oil; yield $87 \%(24 \mathrm{mg}) ;{ }^{1} \mathrm{H}$ NMR $\left(500 \mathrm{MHz}, \mathrm{CDCl}_{3}\right): \delta 7.41(\mathrm{~s}, 1 \mathrm{H})$, 7.19 (s, 1H), 4.95 (s, 2H), 2.07 (s, 3H) , 2.02 (d, J= 0.8 Hz, 3H). ${ }^{13} \mathrm{C}$ NMR (125 $\mathrm{MHz}_{\mathrm{CDCl}}$ ): $\delta 170.9,142.2,140.1,120.5,119.7,56.7,20.9,7.8$. HRMS (ESITOF) m/z: $[\mathrm{M}+\mathrm{H}]^{+}$Calcd for $\mathrm{C}_{8} \mathrm{H}_{11} \mathrm{O}_{3}{ }^{+}$calc 177.0528; Found 177.0533. 


\section{(3-benzylfuran-2-yl)methyl acetate, S7}

OAC

Colorless oil; yield 78\% (19 mg); ${ }^{1} \mathrm{H}$ NMR (500 MHz, $\left.\mathrm{CD}_{3} \mathrm{OD}\right): 7.41-7.40$ (d, J=1.8 $\mathrm{Hz}, 1 \mathrm{H}), 7.27-7.23(\mathrm{~m}, 2 \mathrm{H}), 7.19-7.16(\mathrm{~m}, 3 \mathrm{H}), 6.21(\mathrm{~d}, \mathrm{~J}=1.8 \mathrm{~Hz}, 1 \mathrm{H}), 5.07(\mathrm{~s}, 2 \mathrm{H})$, $3.82(\mathrm{~s}, 2 \mathrm{H}), 2.01(\mathrm{~s}, 3 \mathrm{H}) .{ }^{13} \mathrm{C}$ NMR $\left(125 \mathrm{MHz}, \mathrm{CD}_{3} \mathrm{OD}\right): \delta$ 172.4, 146.8, 143.9, 141.7, 129.5, 129.4, 127.2, 125.4, 113.3, 57.2, 31.6, 20.7. HRMS (ESI-TOF) m/z: [M $+\mathrm{H}]^{+}$Calcd for $\mathrm{C}_{14} \mathrm{H}_{14} \mathrm{O}_{3} \mathrm{H}^{+} 231.1021$; Found 231.0833

\section{(3-methylfuran-2-yl)methyl acetate, S8}

II The NMR data agreed with the reported spectrum of this compound. ${ }^{6}$

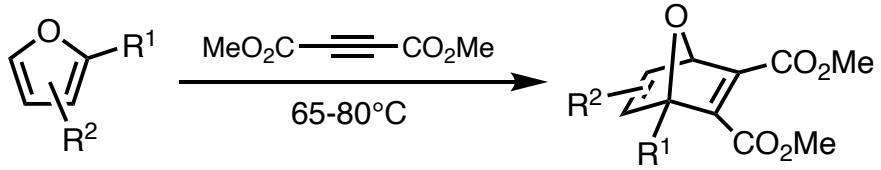

Diels-Alder reactions - general procedure: The furan (1 equiv) and dimethylacetylenedicarboxylate (1.2 equiv) with a few drops of acetonitrile were stirred in a sealed vial for 16 hours at $65-80^{\circ} \mathrm{C}$ (oil bath). The reaction mixture was purified by silica gel chromatography (gradient, $20-60 \%$ ethyl acetate in hexanes).

\section{Dimethyl-1-(acetoxymethyl)-6-methyl-7-oxabicyclo[2.2.1]hepta-2,5-diene-2,3- dicarboxylate, $1 \mathrm{~b}$}

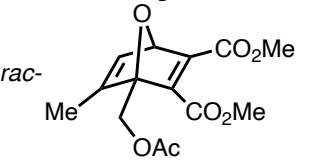

Yellow oil; yield 60\% (5 mg); ${ }^{1} \mathrm{H}$ NMR $\left(500 \mathrm{MHz} \mathrm{CDCl}_{3}\right): \delta$ 6.69- 6.69 (t, J= $1.9 \mathrm{~Hz}, 1 \mathrm{H}), 5.60(\mathrm{~d}, \mathrm{~J}=1.6 \mathrm{~Hz}, 1 \mathrm{H}), 4.90-4.87(\mathrm{~d}, \mathrm{~J}=12.8 \mathrm{~Hz}, 1 \mathrm{H}), 4.63-$ $4.60(\mathrm{~d}, \mathrm{~J}=12.8 \mathrm{~Hz}, 1 \mathrm{H}), 3.83(\mathrm{~s}, 3 \mathrm{H}), 3.80(\mathrm{~s}, 3 \mathrm{H}), 2.08(\mathrm{~s}, 3 \mathrm{H}), 1.97$ (d, J= $1.7 \mathrm{~Hz}, 3 \mathrm{H}) .{ }^{13} \mathrm{C}$ NMR $\left(125 \mathrm{MHz}, \mathrm{CDCl}_{3}\right): \delta 170.5,164.27,162.7,153.4$, 153.2, 152.7, 136.7, 96.2, 83.3, 60.3, 52.3, 20.5, 13.1. HRMS (ESI-TOF) m/z: $[\mathrm{M}+\mathrm{Na}]^{+}$Calcd for $\mathrm{C}_{14} \mathrm{H}_{16} \mathrm{O}_{7} \mathrm{Na}^{+}$calc 319.0794; Found 319.0786.

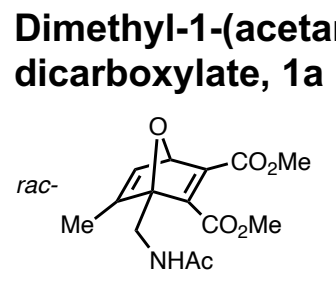

Yellow oil; yield 60\% (50 mg); ${ }^{1} \mathrm{H}$ NMR $\left(500 \mathrm{MHz}, \mathrm{CDCl}_{3}\right): \delta 6.67-6.66(\mathrm{t}, \mathrm{J}=$ $1.9 \mathrm{~Hz}, 1 \mathrm{H}), 5.73(\mathrm{br} \mathrm{s}, 1 \mathrm{H}), 5.57(\mathrm{~d}, \mathrm{~J}=1.6 \mathrm{~Hz}, 1 \mathrm{H}), 4.28-4.24(\mathrm{dd}, \mathrm{J}=6.7$, $14.7 \mathrm{~Hz}, 1 \mathrm{H}), 3.83(\mathrm{~s}, 3 \mathrm{H}), 3.79(\mathrm{~s}, 3 \mathrm{H}), 3.76-3.75(\mathrm{~d}, \mathrm{~J}=4.3 \mathrm{~Hz}, 1 \mathrm{H}), 1.97$ $(\mathrm{s}, 3 \mathrm{H}), 1.94(\mathrm{~d}, \mathrm{~J}=1.8 \mathrm{~Hz}, 3 \mathrm{H}) .{ }^{13} \mathrm{C}$ NMR $\left(125 \mathrm{MHz}, \mathrm{CDCl}_{3}\right): \delta 169.8$, 164.0, 162.5, 153.6, 153.5, 152.9, 136.5, 97.4, 82.8, 52.4, 52.2, 36.7, 22.9, 12.6. HRMS (ESI-TOF) m/z: $[\mathrm{M}+\mathrm{Na}]^{+}$Calcd for $\mathrm{C}_{14} \mathrm{H}_{17} \mathrm{NO}_{6} \mathrm{Na}^{+} 318.0954$; Found 318.0957 .

\section{Dimethyl-1-(acetoxymethyl)-6-benzyl-7-oxabicyclo[2.2.1]hepta-2,5-diene-2,3-} dicarboxylate, 1c

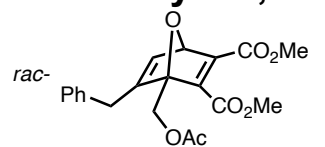

Yellow oil; yield 77\% (40 mg); ${ }^{1} \mathrm{H}$ NMR (500 MHz, $\left.\mathrm{CDCl}_{3}\right): \delta 7.30-7.27$ (m, $2 \mathrm{H}), 7.24-7.21(\mathrm{~m}, 1 \mathrm{H}), 7.13-7.12(\mathrm{~d}, \mathrm{~J}=7.2 \mathrm{~Hz}, 2 \mathrm{H}), 6.57-6.56(\mathrm{~d}, \mathrm{~J}=1.6$ $\mathrm{Hz}, 1 \mathrm{H}), 5.59(\mathrm{~s}, 1 \mathrm{H}), 4.85-4.82(\mathrm{~d}, \mathrm{~J}=12.7 \mathrm{~Hz}, 1 \mathrm{H}), 4.57-4.54(\mathrm{~d}, \mathrm{~J}=12.8$ $\mathrm{Hz}, 1 \mathrm{H}), 3.80(\mathrm{~s}, 3 \mathrm{H}), 3.73(\mathrm{~s}, 3 \mathrm{H}), 3.67-3.57$ (q, J=17.2 Hz, 2H), 2.06 (s, 3H). ${ }^{13} \mathrm{C} \mathrm{NMR}\left(125 \mathrm{MHz}, \mathrm{CDCl}_{3}\right): \delta 170.4,163.7,162.8,157.2,153.5,152.3,137.8,136.5$, 129.0, 128.6, 126.8, 96.1, 83.4, 60.4, 52.4, 52.3, 34.2, 20.5. HRMS (ESI-TOF) m/z: [M + Na] $]^{+}$ Calcd for $\mathrm{C}_{20} \mathrm{H}_{20} \mathrm{O}_{7} \mathrm{Na}^{+} 395.1107$; Found 395.1101. 


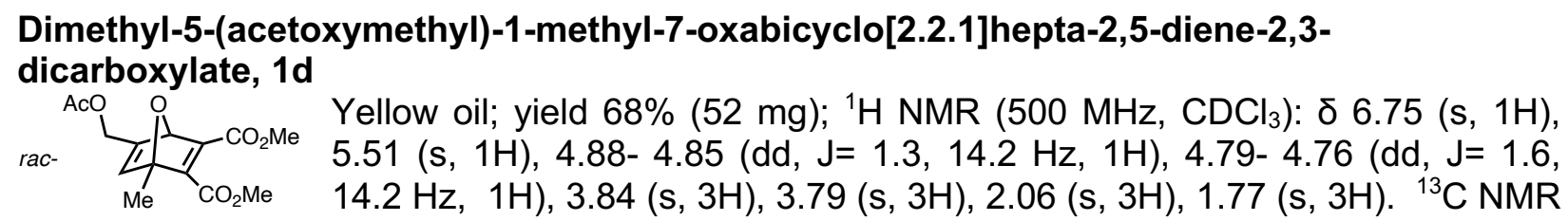
$\left(125 \mathrm{MHz}_{1} \mathrm{CDCl}_{3}\right): \delta 170.4,164.7,162.7,156.2,154.9,150.8,141.2,94.4,84.1,60.0,52.3$, 52.3, 20.7, 15.2. HRMS (ESI-TOF) $\mathrm{m} / \mathrm{z}$ : $[\mathrm{M}+\mathrm{Na}]^{+}$Calcd for $\mathrm{C}_{14} \mathrm{H}_{16} \mathrm{O}_{7} \mathrm{Na}^{+}$calc 319.0794; Found 319.0789 .

\section{Dimethyl-5-(acetoxymethyl)-1-phenyl-7-oxabicyclo[2.2.1] hepta-2,5-diene-2,3- dicarboxylate, $1 \mathrm{f}$}

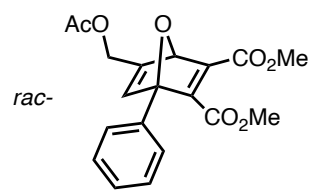

Yellow oil; yield 93\% (107 mg); ${ }^{1} \mathrm{H}$ NMR (500 MHz, $\left.\mathrm{CDCl}_{3}\right)$ : $\delta 7.50-7.48(\mathrm{~m}$, $2 \mathrm{H}), 7.42-7.38(\mathrm{~m}, 3 \mathrm{H}), 7.22-7.21(\mathrm{t}, \mathrm{J}=1.5 \mathrm{~Hz}, 1 \mathrm{H}), 5.74(\mathrm{~s}, 1 \mathrm{H}), 4.96-4.93$ (dd, J=1.5, 14.3 Hz, 1H), 4.88- 4.85 (dd, J=1.6, 14.3 Hz, 1H), 3.81 (s, 3H), $3.64(\mathrm{~s}, 3 \mathrm{H}), 2.09(\mathrm{~s}, 3 \mathrm{H}) .{ }^{13} \mathrm{C}$ NMR $\left(176 \mathrm{MHz} \mathrm{CDCl}_{3}\right): \delta$ 170.5, 164.8, 162.3, 158.4, 155.4, 148.6, 139.2, 133.5, 129.1, 128.6, 126.8, 98.5, 84.4, 60.1, 52.4, 52.3, 20.8. HRMS (ESI-TOF) m/z: $[\mathrm{M}+\mathrm{Na}]^{+}$Calcd for $\mathrm{C}_{19} \mathrm{H}_{18} \mathrm{O}_{7} \mathrm{Na}^{+} 381.0950$; Found 381.0957 .

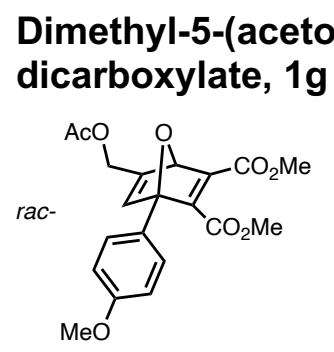

\section{oxymethyl)-1-(4-methoxyphenyl)-7-oxabicyclo[2.2.1] hepta-2,5-diene-2,3-}

Yellow oil; yield 97\% (58 mg); ${ }^{1} \mathrm{H}$ NMR (500 MHz, $\left.\mathrm{CDCl}_{3}\right): \delta 7.43-7.40$ (dt, $\mathrm{J}=2.8,9.6 \mathrm{~Hz}, 2 \mathrm{H}), 7.20(\mathrm{t}, \mathrm{J}=2.8,9.7 \mathrm{~Hz}, 1 \mathrm{H}), 6.93-6.90(\mathrm{~m}, 2 \mathrm{H}), 5.71(\mathrm{~s}$, $1 \mathrm{H}), 4.95-4.92$ (dd, J=1.6, $14.3 \mathrm{~Hz}, 1 \mathrm{H}), 4.87-4.84$ (dd, J= 1.6, $14.3 \mathrm{~Hz}$, $1 \mathrm{H}), 3.82(\mathrm{~s}, 3 \mathrm{H}), 3.80(\mathrm{~s}, 3 \mathrm{H}), 3.66(\mathrm{~s}, 3 \mathrm{H}), 2.09(\mathrm{~s}, 3 \mathrm{H}) \cdot{ }^{13} \mathrm{C}$ NMR $(125$ $\left.\mathrm{MHz}, \mathrm{CDCl}_{3}\right): 170.5,165.0,162.4,160.1,158.3,155.4,148.7,139.1,128.4$, 125.5, 114.01, 98.4, 84.4, 60.1, 55.2, 52.4, 52.3, 20.8. HRMS (ESI-TOF) $\mathrm{m} / \mathrm{z}:[\mathrm{M}+\mathrm{Na}]^{+}$Calcd for $\mathrm{C}_{19} \mathrm{H}_{18} \mathrm{O}_{7} \mathrm{Na}^{+}$411.1056; Found 411.1055.

Dimethyl (1S,4R)-5-(acetoxymethyl)-1-(4-nitrophenyl)-7-oxabicyclo[2.2.1]hepta-2,5-diene2,3-dicarboxylate, $1 \mathrm{~h}$

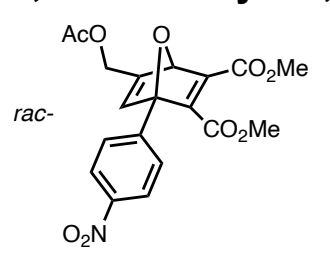

Yellow oil; Yield 76\% (9 mg); ${ }^{1} \mathrm{H}$ NMR (500 MHz, $\left.\mathrm{CDCl}_{3}\right): \delta$ 8.29- $8.27(\mathrm{~m}$, 2H), 7.71- $7.69(\mathrm{~m}, 2 \mathrm{H}), 7.18-7.17(\mathrm{q}, \mathrm{J}=1.4 \mathrm{~Hz}, 1 \mathrm{H}), 5.78(\mathrm{~d}, \mathrm{~J}=0.8 \mathrm{~Hz}$, $1 \mathrm{H}), 4.97-4.93(\mathrm{dd}, \mathrm{J}=1.4,14.5 \mathrm{~Hz}, 1 \mathrm{H}), 4.89-4.86(\mathrm{dd}, \mathrm{J}=1.4,14.5 \mathrm{~Hz}$, $1 \mathrm{H}), 3.83(\mathrm{~d}, \mathrm{~J}=1.2 \mathrm{~Hz}, 3 \mathrm{H}), 3.69(\mathrm{~d}, \mathrm{~J}=1.2 \mathrm{~Hz}, 3 \mathrm{H}), 2.10(\mathrm{~s}, 3 \mathrm{H}) .{ }^{13} \mathrm{C} N M R$ $\left(176 \mathrm{MHz} \mathrm{CDCl}_{3}\right): \delta 170.4,164.1,162.1,156.8,155.8,149.3,148.2,140.5$, 138.4, 127.7, 123.7, 97.2, 84.6, 59.9, 52.6, 52.5, 20.7. HRMS (ESI-TOF) $\mathrm{m} / \mathrm{z}:[\mathrm{M}+\mathrm{Na}]^{+}$Calcd for $\mathrm{C}_{19} \mathrm{H}_{17} \mathrm{NO}_{9} \mathrm{Na}^{+} 426.0801$; Found 426.0795.

\section{Dimethyl-5-(acetoxymethyl)-1-(4-(trifluoromethyl)phenyl)-7-oxabicyclo[2.2.1]hepta-2,5- diene-2,3-dicarboxylate, $1 \mathrm{i}$}

\footnotetext{
rac-

White solid; yield 60\% (16 mg); ${ }^{1} \mathrm{H}$ NMR (500 MHz, $\left.\mathrm{CDCl}_{3}\right): \delta 7.68-7.67$ (d, $\mathrm{J}=8.2 \mathrm{~Hz}, 2 \mathrm{H}), 7.64-7.62(\mathrm{~d}, \mathrm{~J}=8.2 \mathrm{~Hz}, 2 \mathrm{H}), 7.18(\mathrm{t}, \mathrm{J}=1.7 \mathrm{~Hz}, 1 \mathrm{H}), 5.76$ (s, 1H), 4.96- 4.93 (dd, J=1.6, 14.4 Hz, 1H), 4.89-4.86 (dd, J= 1.7, $14.4 \mathrm{~Hz}$, $1 \mathrm{H}), 3.82(\mathrm{~s}, 3 \mathrm{H}), 3.66(\mathrm{~s}, 3 \mathrm{H}), 2.09(\mathrm{~s}, 3 \mathrm{H}) .{ }^{13} \mathrm{C}$ NMR $\left(125 \mathrm{MHz}, \mathrm{CDCl}_{3}\right)$ : $170.4,164.4,162.2,157.4,155.6,149.0,138.7,137.5,131.0,127.1,125.6-$ 125.5, 122.8, 97.6, 84.5, 60.0, 52.5, 52.4, 20.8. HRMS (ESI-TOF) m/z: [M + $\mathrm{Na}]^{+}$Calcd for $\mathrm{C}_{20} \mathrm{H}_{17} \mathrm{~F}_{3} \mathrm{O}_{7} \mathrm{Na}^{+}$449.0824; Found 449.0816.
} 


\section{Dimethyl-5-(acetoxymethyl)-6-methyl-7-oxabicyclo[2.2.1]hepta-2,5-diene-2,3-}

dicarboxylate, 1e

Colorless oil; yield $58 \%(10 \mathrm{mg}) ;{ }^{1} \mathrm{H} N \mathrm{NMR}\left(500 \mathrm{MHz}, \mathrm{CDCl}_{3}\right): \delta 5.60-5.59(\mathrm{~d}$,
$\mathrm{Je}$ (s, 3H). ${ }^{13} \mathrm{C} \mathrm{NMR}\left(176 \mathrm{MHz}, \mathrm{CDCl}_{3}\right): \delta 170.8,163.3,163.1,153.4,151.67,151.5,142.0,88.9$, 86.9, 57.8, 52.4, 20.8, 12.1. HRMS (ESI-TOF) m/z: $[\mathrm{M}+\mathrm{Na}]^{+}$Calcd for $\mathrm{C}_{14} \mathrm{H}_{16} \mathrm{O}_{7} \mathrm{Na}^{+} 319.0794$; Found 319.0786.

\section{Dimethyl-1-(difluoromethyl)-4-(hydroxymethyl)-7-oxabicyclo[2.2.1]hepta-2,5-diene-2,3- dicarboxylate, $1 \mathrm{k}$}

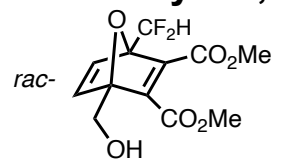

Reaction at $80^{\circ} \mathrm{C}$, yellow oil; yield $60 \%(56 \mathrm{mg}) ;{ }^{1} \mathrm{H}$ NMR $\left(700 \mathrm{MHz}, \mathrm{CDCl}_{3}\right): \delta$ 7.19 (br s, 2H), 6.56, 6.49, 6.41 (3 s, 1H), 4.31 (s, 1H), 3.84 (s, 3H), 3.82 (s, $3 \mathrm{H}), 2.67$ (br s, 1H). ${ }^{13} \mathrm{C}$ NMR $\left(176 \mathrm{MHz} \mathrm{CDCl}_{3}\right): \delta 163.5,163.1,152.5$, $151.7,144.8,141.2,112.8,111.4,110.0,97.9,94.1,94.0,93.8,59.6,52.8$, 52.7. HRMS (ESI-TOF) m/z: [M + Na] ${ }^{+}$Calcd for $\mathrm{C}_{12} \mathrm{H}_{12} \mathrm{~F}_{2} \mathrm{O}_{6} \mathrm{Na}^{+} 313.0494$; Found 313.0496.

\section{Dimethyl-1-fluo
dicarboxylate,}

\section{1}

Reaction at $80^{\circ} \mathrm{C}$ for $24 \mathrm{~h}$, yellow oil; yield $52 \%(13 \mathrm{mg}) ;{ }^{1} \mathrm{H}$ NMR $(500 \mathrm{MHz}$, $\left.\mathrm{CDCl}_{3}\right): \delta 7.16(\mathrm{dd}, \mathrm{J}=2.5,5.5 \mathrm{~Hz}, 1 \mathrm{H}), 7.11(\mathrm{~d}, \mathrm{~J}=5.5 \mathrm{~Hz}, 1 \mathrm{H}), 4.29(\mathrm{~d}, \mathrm{~J}=$ $7.0 \mathrm{~Hz}, 2 \mathrm{H}), 3.88(\mathrm{~s}, 1 \mathrm{H}), 3.83(\mathrm{~s}, 1 \mathrm{H}), 2.66(\mathrm{t}, \mathrm{J}=7.2 \mathrm{~Hz}, 1 \mathrm{H}) .{ }^{13} \mathrm{C}$ NMR $(176$ $\left.\mathrm{MHz}_{\mathrm{CDCl}}\right): \delta 163.1,162.0,151.5,151.3,145.7-145.6,141.0,140.9,122.0$, 120.5, 91.0, 59.7, 52.9, 52.7. HRMS (ESI-TOF) $\mathrm{m} / \mathrm{z}:[\mathrm{M}+\mathrm{Na}]^{+}$Calcd for $\mathrm{C}_{11} \mathrm{H}_{11} \mathrm{~F}_{1} \mathrm{O}_{6} \mathrm{Na}^{+}$ 281.0432; Found 281.0428.

\section{Dimethyl-1-(((tert-butoxycarbonyl)amino)methyl)-4-(trifluoromethyl)-7- oxabicyclo[2.2.1] hepta-2,5-diene-2,3-dicarboxylate, S9}

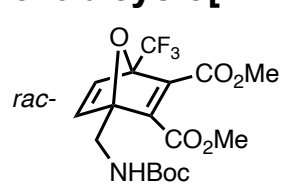

Reaction at $80^{\circ} \mathrm{C}$ for 6 days, yellow oil; yield $40 \%$ (15 mg); ${ }^{1} \mathrm{H}$ NMR $(400 \mathrm{MHz}$, $\left.\mathrm{CDCl}_{3}\right): \delta 7.14-7.19(\mathrm{~m}, 2 \mathrm{H}), 4.99(\mathrm{br} \mathrm{s}, 1 \mathrm{H}), 4.10(\mathrm{~m}, 1 \mathrm{H}$, overlap with EtOAc), 3.97 (dd, J = 6.2, $15.2 \mathrm{~Hz}, 1 \mathrm{H}$ ), 3.83 (s, 3H), 3.78 (s, 3H), 1.42 (s, 9H). ${ }^{13} \mathrm{C}$ NMR $\left(100 \mathrm{MHz}, \mathrm{CDCl}_{3}\right): \delta 162.7,162.2,155.8,152.6,151.3,145.9$, 140.8, 123.3, 120.5, 97.3, 93.2, 92.9, 91.4, 80.0, 52.9, 52.8, 39.1, 28.4. HRMS (ESI-TOF) m/z: [M + Na] $]^{+}$Calcd for $\mathrm{C}_{17} \mathrm{H}_{20} \mathrm{~F}_{3} \mathrm{~N}_{1} \mathrm{O}_{7} \mathrm{Na}^{+}$430.1090; Found 430.1087.

Dimethyl-1-(hydroxymethyl)-4-(6-(trifluoromethyl)pyridin-3-yl)-7-oxabicyclo[2.2.1]hepta2,5-diene-2,3-dicarboxylate, $\mathbf{S 1 0}$

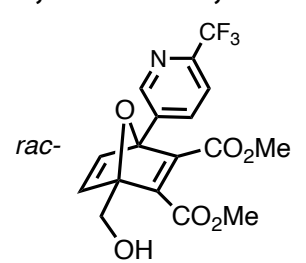

Reaction at $80^{\circ} \mathrm{C}$ for 2 days, yellow oil; yield $91 \%$ (39 mg); ${ }^{1} \mathrm{H}$ NMR (400 $\left.\mathrm{MHz}, \mathrm{CDCl}_{3}\right): \delta 8.90(\mathrm{~d}, \mathrm{~J}=2.0 \mathrm{~Hz}, 1 \mathrm{H}), 8.06(\mathrm{dd}, \mathrm{J}=1.7,8.1 \mathrm{~Hz}, 1 \mathrm{H}), 7.75$ $(\mathrm{d}, \mathrm{J}=8.2 \mathrm{~Hz}, 1 \mathrm{H}), 7.45(\mathrm{~d}, \mathrm{~J}=5.2 \mathrm{~Hz}, 1 \mathrm{H}), 7.29(\mathrm{~d}, \mathrm{~J}=5.2 \mathrm{~Hz}, 1 \mathrm{H}), 4.41(\mathrm{~d}$, $\mathrm{J}=6.3 \mathrm{~Hz}, 2 \mathrm{H}), 3.84(\mathrm{~s}, 3 \mathrm{H}), 3.68(\mathrm{~s}, 3 \mathrm{H}), 2.98(\mathrm{t}, \mathrm{J}=6.7 \mathrm{~Hz}, 1 \mathrm{H}) .{ }^{13} \mathrm{C} \mathrm{NMR}$ $\left(100 \mathrm{MHz}, \mathrm{CDCl}_{3}\right): \delta 163.7,163.6,157.5,149.9,148.7,148.3,144.9,144.5$, 136.1, 132.9, 122.7, 120.1, 120.1, 97.3, 94.1, 59.9, 52.8, 52.5. HRMS (ESITOF) $\mathrm{m} / \mathrm{z}:[\mathrm{M}+\mathrm{Na}]^{+}$Calcd for $\mathrm{C}_{17} \mathrm{H}_{14} \mathrm{~F}_{3} \mathrm{~N}_{1} \mathrm{O}_{6} \mathrm{H}^{+} 386.0851$; Found 386.0855.

\section{Dimethyl-1-(3,5-bis(trifluoromethyl)phenyl)-4-(hydroxymethyl)-7-oxabicyclo[2.2.1]hepta-} 2,5-diene-2,3-dicarboxylate, S11

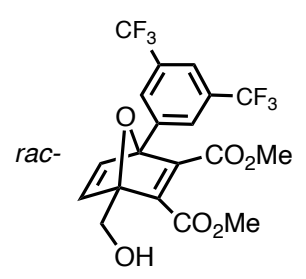

Reaction at $80^{\circ} \mathrm{C}$ for 6 days, yellow oil; yield $56 \%$ (31 mg); ${ }^{1} \mathrm{H}$ NMR (400 $\mathrm{MHz} \mathrm{CDCl}_{3}$ ): $\delta 7.99$ (br s, 2H), 7.91 (br s, 1H), 7.41 (d, J = 5.2 Hz), 7.29 (d, J $=5.2 \mathrm{~Hz}, 1 \mathrm{H}), 4.41(\mathrm{~d}, \mathrm{~J}=6.2 \mathrm{~Hz}), 3.83(\mathrm{~s}, 3 \mathrm{H}), 3.66(\mathrm{~s}, 3 \mathrm{H}), 2.90(\mathrm{t}, \mathrm{J}=6.7$ $\mathrm{Hz}, 1 \mathrm{H}) .{ }^{13} \mathrm{C}$ NMR $\left(100 \mathrm{MHz}, \mathrm{CDCl}_{3}\right): \delta 164.0,163.7,158.4,149.5,145.3$, 
144.8, 136.6, 132.3, 131.9, 127.2, 124.5, 122.9, 121.8, 97.3, 95.4, 60.1, 53.0, 52.5. HRMS (ESITOF) $\mathrm{m} / \mathrm{z}:[\mathrm{M}+\mathrm{H}]^{+}$Calcd for $\mathrm{C}_{19} \mathrm{H}_{14} \mathrm{~F}_{6} \mathrm{O}_{6} \mathrm{H}^{+}$calc 453.0773; Found 453.0771.

\section{Dimethyl-1-(3,5-bis(trifluoromethyl)phenyl)-4-(hydroxymethyl)-7-oxabicyclo[2.2.1]hepta-} 2,5-diene-2,3-dicarboxylate, 1p

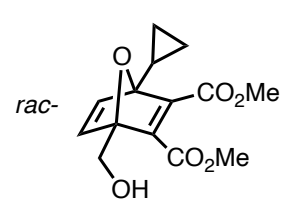

Reaction at $75^{\circ} \mathrm{C}$, yellow oil; yield $94 \%(85 \mathrm{mg}) ;{ }^{1} \mathrm{H}$ NMR $\left(400 \mathrm{MHz}, \mathrm{CDCl}_{3}\right): \delta$ $7.04(\mathrm{dd}, \mathrm{J}=0.76,5.3 \mathrm{~Hz}, 1 \mathrm{H}), 6.77(\mathrm{~d}, \mathrm{~J}=5.3 \mathrm{~Hz}, 1 \mathrm{H}), 4.21(\mathrm{~d}, \mathrm{~J}=4.9 \mathrm{~Hz})$, $3.81(\mathrm{~s}, 3 \mathrm{H}), 3.73(\mathrm{~s}, 3 \mathrm{H}), 2.98(\mathrm{t}, \mathrm{J}=6.6 \mathrm{~Hz}, 1 \mathrm{H}), 1.42-1.52(\mathrm{~m}, 1 \mathrm{H}), 0.56-$ $0.71(\mathrm{~m}, 3 \mathrm{H}), 0.47-0.53(\mathrm{~m}, 1 \mathrm{H}) .{ }^{13} \mathrm{C}$ NMR $\left(100 \mathrm{MHz}, \mathrm{CDCl}_{3}\right): \delta 165.2,163.9$, 159.8, 148.9, 144.8, 143.6, 97.1, 95.8, 60.1, 52.5, 52.3, 9.3, 2.4, 2.4. HRMS (ESI-TOF) m/z: [M + Na] ${ }^{+}$Calcd for $\mathrm{C}_{14} \mathrm{H}_{16} \mathrm{O}_{6} \mathrm{Na}^{+} 303.0845$; Found 303.0842 .

\section{Dimethyl-1-(2-hydroxyoxetan-2-yl)-4-methyl-7-oxabicyclo[2.2.1]hepta-2,5-diene-2,3- dicarboxylate, 1q}

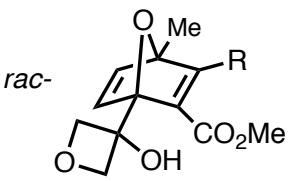

Reaction at $70^{\circ} \mathrm{C}$, yellow oil; yield $87 \%(297 \mathrm{mg}) ;{ }^{1} \mathrm{H}$ NMR $\left(500 \mathrm{MHz}, \mathrm{CDCl}_{3}\right)$ : $\delta 7.18(\mathrm{~d}, \mathrm{~J}=5.2 \mathrm{~Hz}, 1 \mathrm{H}), 6.97(\mathrm{~d}, \mathrm{~J}=5.2 \mathrm{~Hz}, 1 \mathrm{H}), 4.73(\mathrm{~d}, \mathrm{~J}=7.3 \mathrm{~Hz}, 1 \mathrm{H})$, $4.62(\mathrm{~d}, \mathrm{~J}=7.2 \mathrm{~Hz}, 2 \mathrm{H}), 4.56(\mathrm{~d}, \mathrm{~J}=7.0 \mathrm{~Hz}, 1 \mathrm{H}), 3.72(\mathrm{~s}, 3 \mathrm{H}), 3.65(\mathrm{~s}, 3 \mathrm{H})$, $1.69(\mathrm{~s}, 3 \mathrm{H}) .{ }^{13} \mathrm{C}$ NMR $\left(125 \mathrm{MHz} \mathrm{CDCl}_{3}\right): \delta 164.4,164.2,158.6,149.9$, 148.1, 143.0, 97.3, 92.8, 80.7, 80.1, 72.0, 52.6, 52.2, 15.0. HRMS (ESI-TOF) $\mathrm{m} / \mathrm{z}:[\mathrm{M}+\mathrm{Na}]^{+}$Calcd for $\mathrm{C}_{14} \mathrm{H}_{16} \mathrm{O}_{7} \mathrm{Na}^{+}$calc 319.0788; Found 319.0792.

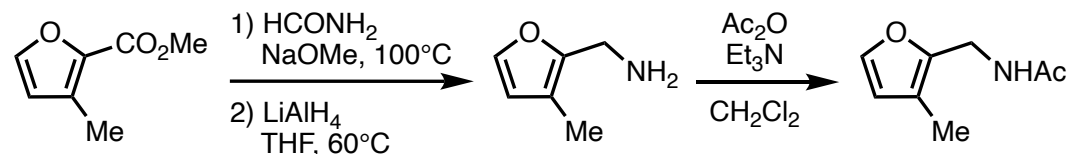

N-((3-methylfuran-2-yl)methyl)acetamide, S12: This compound was prepared according to a literature procedure. ${ }^{7}$ The NMR data agreed with the reported spectrum of this compound.

(3-methylfuran-2-yl)methanol, S13: This compound was prepared according to a literature procedure. ${ }^{8}$ The NMR data agreed with the reported spectrum of this compound.

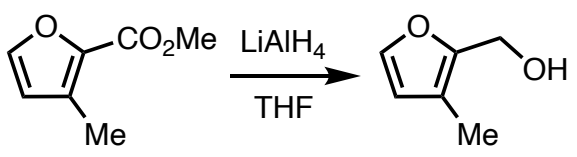

3-Hydroxymethylfurans - general procedure: Following the published procedure, ${ }^{9}$ the enone $(1$ equiv) was dissolved in dichloromethane. A solution of $\mathrm{BF}_{3} \cdot \mathrm{OEt}_{2}(1 \% \mathrm{~mol})$ in dichloromethane was added. The solution was stirred at room<smiles>[R7]C(C(=O)[R7](Br)OCC)=C1COC1</smiles>
temperature for $2 \mathrm{~min}$. The reaction mixture was purified by silica gel chromatography $(50 \%$ diethyl ether in hexanes).

(5-methylfuran-3-yl)methanol (S14), (5-phenylfuran-3-yl)methanol (S15), (4-methylfuran-3yl)methanol (S16)<smiles>Cc1cc(CO)co1</smiles><smiles>OCc1coc(-c2ccccc2)c1</smiles><smiles>Cc1cocc1CO</smiles>

The NMR data agreed with the reported spectra of these compounds. ${ }^{9}$ 


\section{(5-(4-methoxyphenyl)furan-3-yl)methanol, S17}

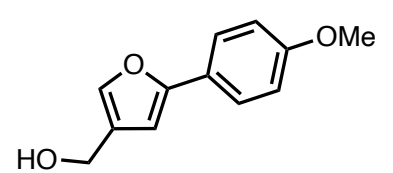

205.0865; Found 205.0863.

\section{(5-(4-nitrophenyl)furan-3-yl)methanol, S18}

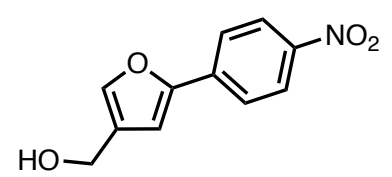

Yellow solid; yield 90\% (27 mg); ${ }^{1} \mathrm{H}$ NMR (500 MHz, $\left.\mathrm{CDCl}_{3}\right): \delta 8.25-$ 8.24 (d, J=7.5 Hz, 2H), 7.78- 7.76 (d, J= 7.4 Hz, 2H), 7.55 (s, 1H), 6.92 (s, 1H), $4.63(\mathrm{~s}, 2 \mathrm{H}), 1.63(\mathrm{br} \mathrm{s}, 3 \mathrm{H}) .{ }^{13} \mathrm{C} \mathrm{NMR}\left(125 \mathrm{MHz}, \mathrm{CDCl}_{3}\right): \delta$ $152.4,146.5,141.2,136.2,127.9,124.3,124.0$, 108.9, 56.6. HRMS (ESI-TOF) $\mathrm{m} / \mathrm{z}:[\mathrm{M}+\mathrm{Na}]^{+}$Calcd for $\mathrm{C}_{11} \mathrm{H}_{9} \mathrm{NO}_{4} \mathrm{Na}^{+} 242.0429$; Found

242.0424 .

(5-(4-(trifluoromethyl)phenyl)furan-3-yl)methanol, S19<smiles>OCc1coc(-c2ccc(C(F)(F)F)cc2)c1</smiles>

found 243.0631 .

White solid; yield 96\% (105 mg); ${ }^{1} \mathrm{H}$ NMR (500 MHz, $\left.\mathrm{CDCl}_{3}\right)$ : 7.74-7.72 $(\mathrm{d}, \mathrm{J}=8.2 \mathrm{~Hz}, 2 \mathrm{H}), 7.63-7.61(\mathrm{~d}, \mathrm{~J}=8.4 \mathrm{~Hz}, 2 \mathrm{H}), 7.49(\mathrm{~s}, 1 \mathrm{H}), 6.81$ (s, $1 \mathrm{H}), 4.61$ (s, 2H), 1.74 (br s, $1 \mathrm{H}) .{ }^{13} \mathrm{C}$ NMR (125 MHz, $\left.\mathrm{CDCl}_{3}\right): \delta 153.2$, 140.2, 133.7, 129.3, 129.0, 127.5, 125.7-125.7, 123.8, 107.0, 56.7. HRMS (ESI-TOF) m/z: [M + H] $]^{+}$Calcd for $\mathrm{C}_{12} \mathrm{H}_{9} \mathrm{~F}_{3} \mathrm{O}_{2} \mathrm{H}^{+}$calc 243.0633;

Wittig reaction - general procedure: Following the published procedure ${ }^{9}$ the phosphorane $(1.4$ equiv) was dissolved in dry dichloromethane. 3oxetanone was added (1.0 equiv). The mixture was stirred at room temperature for 16 hours. The reaction mixture was purified by silica gel chromatography $(50 \%$ diethyl ether in hexanes).

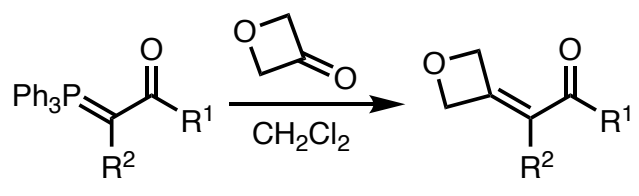

\section{1-(oxetan-3-ylidene)propan-2-one (S20), 1-(oxetan-3-ylidene)propan-2-one (S21)}

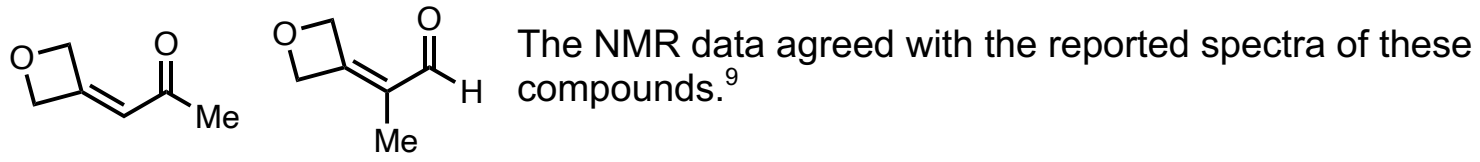

\section{1-(4-methoxyphenyl)-2-(oxetan-3-ylidene)ethan-1-one, S22}

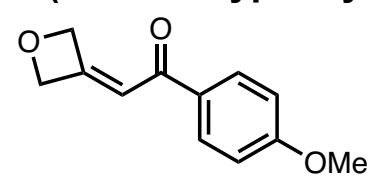

Pink solid; yield 98\% (185 mg); ${ }^{1} \mathrm{H}$ NMR (500 MHz, $\left.\mathrm{CDCl}_{3}\right): \delta$ 7.91- 7.88 (dt, J=2.7, 9.7 Hz, 2H), 6.95- $6.92(\mathrm{~m}, \mathrm{~J}=2.7,9.7 \mathrm{~Hz}, 2 \mathrm{H}), 6.78-6.77$ (p, $\mathrm{J}=2.3 \mathrm{~Hz}, 1 \mathrm{H}), 5.67-5.65(\mathrm{~m}, 2 \mathrm{H}), 5.40-5.38(\mathrm{~m}, 2 \mathrm{H}), 3.85(\mathrm{~s}, 3 \mathrm{H}) .{ }^{13} \mathrm{C}$ NMR (125 MHz, $\left.\mathrm{CDCl}_{3}\right): \delta 159.2,154.9,138.6,127.1,125.3,123.8$, 114.1, 103.5, 56.9, 55.3. HRMS (ESI-TOF) $\mathrm{m} / \mathrm{z}$ : $[\mathrm{M}+\mathrm{Na}]^{+}$Calcd for $\mathrm{C}_{12} \mathrm{H}_{12} \mathrm{O}_{3} \mathrm{Na}^{+} 227.0684$; Found 227.0681.

\section{1-(4-nitrophenyl)-2-(oxetan-3-ylidene)ethan-1-one, S23}

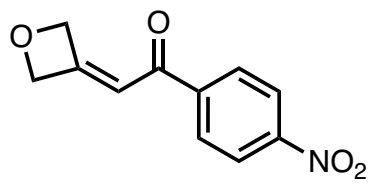

Yellow solid; yield 45\% (102 mg); ${ }^{1} \mathrm{H}$ NMR (500 MHz, $\left.\mathrm{CDCl}_{3}\right): \delta 8.34-$ $8.32(\mathrm{dt}, \mathrm{J}=2.2,9.1 \mathrm{~Hz}, 2 \mathrm{H}), 8.08-8.06(\mathrm{dt}, \mathrm{J}=2.2,9.1 \mathrm{~Hz}, 2 \mathrm{H}), 6.85-$ $6.83(\mathrm{p}, \mathrm{J}=2.3 \mathrm{~Hz}, 1 \mathrm{H}), 5.69-5.67(\mathrm{~m}, 2 \mathrm{H}), 5.46-5.44(\mathrm{~m}, 2 \mathrm{H}) .{ }^{13} \mathrm{C}$ NMR (125 MHz, $\left.\mathrm{CDCl}_{3}\right): \delta 187.1,164.5,150.2,142.1,129.1,123.9$, 112.9, 82.5, 79.2. HRMS (ESI-TOF) $\mathrm{m} / \mathrm{z}:[\mathrm{M}+\mathrm{Na}]^{+}$Calcd for 
2-(oxetan-3-ylidene)-1-(4-(trifluoromethyl)phenyl)ethan-1-one, S24<smiles>O=C(C=C1COC1)c1ccc(C(F)(F)F)cc1</smiles>
White solid; yield $43 \%(109 \mathrm{mg}) ;{ }^{1} \mathrm{H}$ NMR $\left(500 \mathrm{MHz}, \mathrm{CDCl}_{3}\right)$ : $\delta 8.02-$ $8.01(\mathrm{~d}, \mathrm{~J}=8.1 \mathrm{~Hz}, 2 \mathrm{H}), 7.75-7.73(\mathrm{~d}, \mathrm{~J}=8.3 \mathrm{~Hz}, 2 \mathrm{H}), 6.83-6.82(\mathrm{p}, \mathrm{J}=$ $2.3 \mathrm{~Hz}, 1 \mathrm{H}), 5.69-5.67(\mathrm{~m}, 2 \mathrm{H}), 5.45-5.43(\mathrm{~m}, 2 \mathrm{H}) .{ }^{13} \mathrm{C} \mathrm{NMR}(125 \mathrm{MHz}$, $\mathrm{CD}_{3} \mathrm{OD}$ ): $\delta 184.5,164.4,141.9,135.3,130.0,126.9-124.1,114.5,83.5$, 243.0633; Found 243.0631.

80.6. HRMS (ESI-TOF) $\mathrm{m} / \mathrm{z}$ : $[\mathrm{M}+\mathrm{H}]^{+}$Calcd for $\mathrm{C}_{12} \mathrm{H}_{9} \mathrm{~F}_{3} \mathrm{O}_{2} \mathrm{H}^{+}$calc

2-(oxetan-3-ylidene)-1-phenylethan-1-one, S25: This compound was prepared according to a literature procedure. ${ }^{9}$ The NMR data agreed with the reported spectrum of this compound.

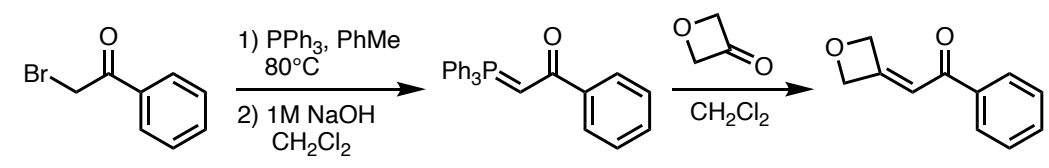

1-(4-Methoxyphenyl)-2-(triphenylphosphoranylidene)ethenone, 1-(4(Trifluoromethyl)phenyl)-2-(triphenylphosphoranylidene)ethenone, S26:

These compounds were prepared according to a literature procedure..$^{10}$ The NMR data agreed with their reported spectra.

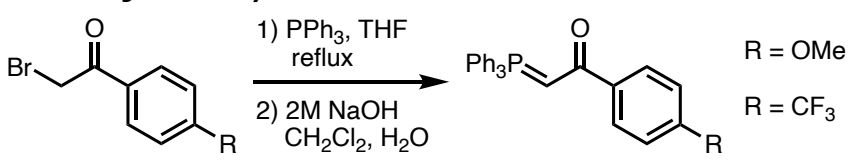

1-(4-(Nitro)phenyl)-2-(triphenylphosphoranylidene)ethenone, S27: This compound was prepared according to a literature procedure. ${ }^{11}$ The NMR data agreed with the reported spectrum of this compound.

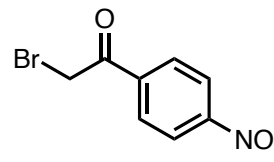

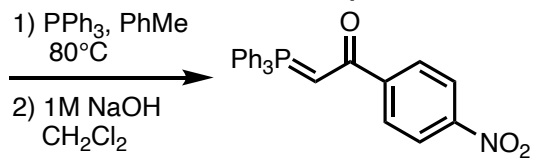

3-benzylfuran-2-carbaldehyde, S28: 3bromo-2-furfuryl aldehyde (1 equiv), benzylboronic acid pinacol ester (1.2 equiv), potassium carbonate (3 equiv) and bis(triphenylphosphine)palladium(II)

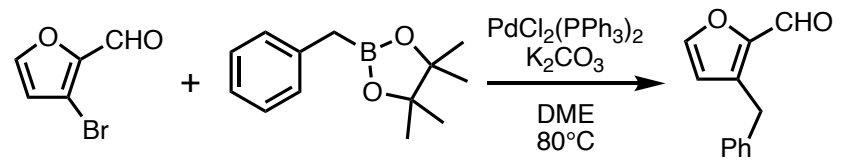
dichloride $(10 \% \mathrm{~mol})$ were added to a vial. The vial was evacued and backfilled with nitrogen three times. The solvent, dimethoxyethane, was added as well as water and the solution was heated at $80^{\circ} \mathrm{C}$ (oil bath). Saturated $\mathrm{NH}_{4} \mathrm{Cl}$ was added until the solution reached a $\mathrm{pH}$ of 7 and the organic layer was extracted with ethyl acetate three times. The combined organic layer was washed with brine, dried over sodium sulfate and the solvent was removed under vacuum. The residue obtained was purified by silica gel chromatography (10\% ethyl acetate in hexanes) to give a yellow oil $(58 \mathrm{mg}, 30 \%$ yield).

${ }^{1} \mathrm{H}$ NMR $\left(500 \mathrm{MHz}, \mathrm{CDCl}_{3}\right): \delta 9.81(\mathrm{~s}, 1 \mathrm{H}), 7.55-7.55(\mathrm{~d}, \mathrm{~J}=1.5 \mathrm{~Hz}, 1 \mathrm{H}), 7.33-7.30(\mathrm{~m}, 2 \mathrm{H})$, 7.25- $7.22(\mathrm{~m}, 3 \mathrm{H}), 6.37-6.37(\mathrm{~d}, \mathrm{~J}=1.6 \mathrm{~Hz}, 1 \mathrm{H}), 4.17(\mathrm{~s}, 2 \mathrm{H}) .{ }^{13} \mathrm{C} \mathrm{NMR}\left(125 \mathrm{MHz}, \mathrm{CDCl}_{3}\right): \delta$ 178.4, 148.3, 147.3, 138.6, 128.7, 128.6, 126.7, 114.6, 30.9. HRMS (ESI-TOF) m/z: [M + H] ${ }^{+}$ Calcd for $\mathrm{C}_{12} \mathrm{H}_{11} \mathrm{O}_{2} \mathrm{H}^{+}$187.0759; Found 187.0754.

(3-benzylfuran-2-yl)methanol, $\quad$ S29: 3-benzylfuran-2carbaldehyde (1 equiv) was dissolved in methanol and sodium borohydride (1.5 equiv) was added. The solution was stirred at room temperature for two hours. Saturated $\mathrm{NH} 4 \mathrm{Cl}$ was added

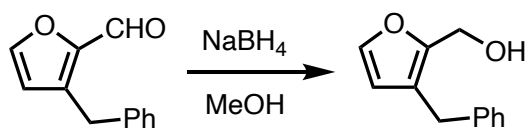
and the organic layer was extracted with dichloromethane three times. The combined organic 
layer was washed with brine, dried over sodium sulfate and the solvent was removed under vacuum. The residue obtained was purified by silica gel chromatography (gradient, 10-30\% ethyl acetate in hexanes) to give a colorless oil (32 $\mathrm{mg}, 70 \%$ yield.)

${ }^{1} \mathrm{H}$ NMR $\left(500 \mathrm{MHz}, \mathrm{CDCl}_{3}\right): 7.33-7.33(\mathrm{~d}, \mathrm{~J}=1.8 \mathrm{~Hz}, 1 \mathrm{H}), 7.31-7.28(\mathrm{~m}, 2 \mathrm{H}), 7.22-7.19(\mathrm{~m}$, $3 \mathrm{H}), 6.20-6.20(\mathrm{~d}, \mathrm{~J}=1.8 \mathrm{~Hz}, 1 \mathrm{H}), 4.60(\mathrm{~s}, 2 \mathrm{H}), 3.81(\mathrm{~s}, 2 \mathrm{H}) \cdot{ }^{13} \mathrm{C}$ NMR $\left(125 \mathrm{MHz}, \mathrm{CDCl}_{3}\right): \delta$ 149.6, 141.9, 140.3, 128.5, 128.3, 126.2, 121.4, 112.3, 55.4, 30.9. HRMS (ESI-TOF) m/z: [M + $\mathrm{Na}]^{+}$Calcd for $\mathrm{C}_{12} \mathrm{H}_{12} \mathrm{O}_{2} \mathrm{Na}^{+} 211.0735$; Found 211.0736.

Activation of 2-hydroxymethyl group - general procedure: A solution of di(N-succinimidyl) carbonate (DSC, 1.5 equiv) and triethylamine (2 equiv) in acetonitrile were stirred for 5 minutes at $0^{\circ} \mathrm{C}$. Then a solution of OND alcohol (1 equiv) in acetonitrile was added dropwise at $0^{\circ} \mathrm{C}$ to the reaction mixture. The ice bath was removed and the reaction mixture was stirred until the consumption of OND starting material (as monitored by TLC, $3 \mathrm{~h})$. The solvent was removed under vacuum and the residue obtained was purified by silica gel chromatography (gradient, $30-70 \%$ ethyl acetate in
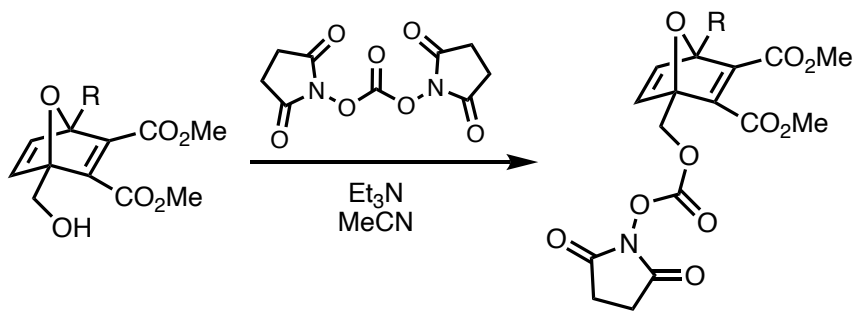
hexanes)

Dimethyl (1R,4R)-1-(difluoromethyl)-4-((((2,5-dioxopyrrolidin-1yl)oxy)carbonyl)oxy)methyl)-7-oxabicyclo[2.2.1] hepta-2,5-diene-2,3dicarboxylate, $\mathbf{S 3 0}$

White solid; yield 43\% (47 mg); ${ }^{1} \mathrm{H}$ NMR $\left(500 \mathrm{MHz}, \mathrm{CDCl}_{3}\right): \delta 7.24-7.23(\mathrm{~d}$, $\mathrm{J}=5.3 \mathrm{~Hz}, 1 \mathrm{H}), 7.16-7.15(\mathrm{~d}, \mathrm{~J}=5.2 \mathrm{~Hz}, 1 \mathrm{H}), 6.55,6.44,6.34(3 \mathrm{~s}, 1 \mathrm{H})$, $5.11-5.04(\mathrm{q}, \mathrm{J}=10.6 \mathrm{~Hz}, 2 \mathrm{H}), 3.82(\mathrm{~s}, 3 \mathrm{H}), 3.81(\mathrm{~s}, 3 \mathrm{H}), 2.80(\mathrm{~s}, 4 \mathrm{H}) .{ }^{13} \mathrm{C}$ NMR $\left(125 \mathrm{MHz} \mathrm{CDCl}_{3}\right): \delta 168.4,162.9,162.1,152.7,151.7,1511,149.8$, 143.6, 141.9, 113.1, 111.2, 109.2, 94.2, 66.4, 52.8, 58.8, 25.3. HRMS (ESITOF) $\mathrm{m} / \mathrm{z}:[\mathrm{M}+\mathrm{H}]^{+}$Calcd for $\mathrm{C}_{17} \mathrm{H}_{15} \mathrm{~F}_{2} \mathrm{~N}_{1} \mathrm{O}_{10} \mathrm{H}^{+}$432.0742; Found 432.0745 .

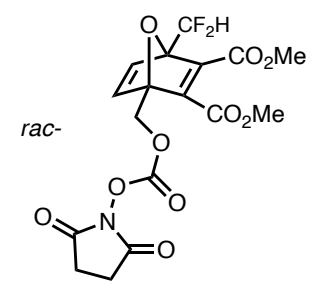

Dimethyl (1R,4S)-1-((((2,5-dioxopyrrolidin-1yl)oxy)carbonyl)oxy)methyl)-4-(6-(trifluoromethyl)pyridin-3-yl)-7oxabicyclo[2.2.1] hepta-2,5-diene-2,3-dicarboxylate, S31

Yellow oil; yield 59\% (32 mg); ${ }^{1} \mathrm{H}$ NMR $\left(400 \mathrm{MHz}, \mathrm{CDCl}_{3}\right): \delta 8.89(\mathrm{~d}, \mathrm{~J}=2.0$ $\mathrm{Hz}, 1 \mathrm{H}), 8.07$ (d, J = 1.7, 8.2 Hz), $7.75(\mathrm{~d}, \mathrm{~J}=8.2 \mathrm{~Hz}, 1 \mathrm{H}), 7.49$ (d, J = 5.2 $\mathrm{Hz}, 1 \mathrm{H}), 7.22(\mathrm{~d}, \mathrm{~J}=5.2 \mathrm{~Hz}, 1 \mathrm{H}), 5.19(\mathrm{~s}, 2 \mathrm{H}), 3.84(\mathrm{~s}, 3 \mathrm{H}), 3.68(\mathrm{~s}, 3 \mathrm{H})$, $2.83(\mathrm{~s}, 4 \mathrm{H}) .{ }^{13} \mathrm{C}$ NMR $\left(176 \mathrm{MHz}, \mathrm{CDCl}_{3}\right): \delta 168.2,163.6,162.3,157.6$, 151.4, 148.3- 148.2, 145.4, 143.4, 136.2, 132.4, 125.9, 123.5, 120.3, 109.7, 94.4, 93.7, 66.8, 52.9, 52.7, 25.4. HRMS (ESI-TOF) m/z: $[\mathrm{M}+\mathrm{H}]^{+}$Calcd for $\mathrm{C}_{22} \mathrm{H}_{17} \mathrm{~F}_{3} \mathrm{~N}_{2} \mathrm{O}_{10} \mathrm{H}^{+}$527.0914; Found 527.0917.

Dimethyl-1-(3,5-bis(trifluoromethyl)phenyl)-4-((((2,5-dioxopyrrolidin-1yl)oxy)carbonyl)oxy)methyl)-7-oxabicyclo[2.2.1] hepta-2,5-diene-2,3dicarboxylate, S32

Yellow oil; Yield 49\% (19 mg); ${ }^{1} \mathrm{H}$ NMR (400 MHz, CDCl 3 ): $\delta 8.02$ (br s, 2H), $7.95(\mathrm{br} \mathrm{s}, 1 \mathrm{H}), 7.50(\mathrm{~d}, \mathrm{~J}=5.2 \mathrm{~Hz}, 1 \mathrm{H}), 7.27(\mathrm{t}, \mathrm{J}=5.2 \mathrm{~Hz}, 1 \mathrm{H}), 5.23(\mathrm{~s}$, $2 \mathrm{H}), 3.87(\mathrm{~s}, 3 \mathrm{H}), 3.70(\mathrm{~s}, 3 \mathrm{H}), 2.86(\mathrm{~s}, 4 \mathrm{H}) .{ }^{13} \mathrm{C} \mathrm{NMR}\left(176 \mathrm{MHz} \mathrm{CDCl}_{3}\right): \delta$ 168.2, 163.6, 162.2, 158.1, 151.4, 147.9, 145.5, 144.6, 135.9, 132.1- 132.9, 127.1, 123.79, 123.0, 122.2, 95.5, 93.7, 66.9, 52.9, 52.5, 25.4. HRMS (ESITOF) $\mathrm{m} / \mathrm{z}$ : $[\mathrm{M}+\mathrm{H}]^{+}$Calcd for $\mathrm{C}_{24} \mathrm{H}_{17} \mathrm{~F}_{6} \mathrm{~N}_{1} \mathrm{O}_{10} \mathrm{H}^{+}$594.0835; Found 594.0838.

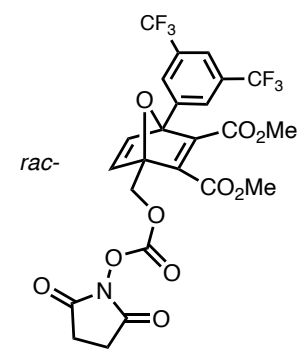



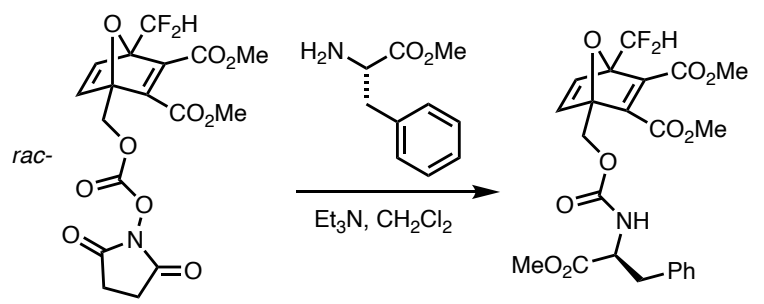

\section{Dimethyl-1-(difluoromethyl)-4-(((((S)-1- methoxy-1-oxo-3-phenylpropan-2- yl)carbamoyl)oxy)methyl)-7-} oxabicyclo[2.2.1]hepta-2,5-diene-2,3-

dicarboxylate, $1 \mathrm{j}$ : A solution of phenylalanine methyl ester hydrochloride (1 equiv) and triethylamine ( 2.5 equiv) in dichloromethane was cooled to $-20^{\circ} \mathrm{C}$. Then a solution of OND N-hydroxysuccinimidyl carbonate (1.1 to 1.2 equiv) in dichloromethane was added dropwise and the reaction mixture was allowed to reach to room temperature in about $1.5 \mathrm{~h}$. Solvent was removed under vaccum and the residue obtained was patitioned between ethyl acetate and water. The organic layer was separated, washed with brine, dried over sodium sulfate and concentrated to obtain the crude product which was purified by silica gel chromatography ( $40 \%$ ethyl acetate in hexanes) and yield product as a pale yellow solid (37 mg, 69\%, mixture of diastereomers).

${ }^{1} \mathrm{H} \mathrm{NMR}\left(700 \mathrm{MHz}, \mathrm{CDCl}_{3}\right): \delta$ 7.27-7.31 (m, 2H), 7.23-7.26 (m, 1H), $7.20(\mathrm{dd}, \mathrm{J}=1.2,5.4 \mathrm{~Hz})$, 7.07-7.14 (m, 3H), 6.60, 6.59, 6.53, 6.52, 6.45, $6.44(6 \mathrm{~s}, 1 \mathrm{H}), 5.29,5.27(2 \mathrm{~d}, \mathrm{~J}=8.3 \mathrm{~Hz}, 1 \mathrm{H})$, 4.84, $4.83(2 \mathrm{~s}, 2 \mathrm{H}), 4.60-4.66(\mathrm{~m}, 1 \mathrm{H}), 3.84,3.83(2 \mathrm{~s}, 3 \mathrm{H}), 3.78,3.75(2 \mathrm{~s}, 3 \mathrm{H}), 3.72,3.71(2 \mathrm{~s}$, $3 \mathrm{H}), 3.14,3.12(2 \mathrm{~d}, \mathrm{~J}=5.7 \mathrm{~Hz}, 1 \mathrm{H}), 3.17-3.09(\mathrm{~m}, 1 \mathrm{H}) .{ }^{13} \mathrm{C} \mathrm{NMR}\left(176 \mathrm{MHz}, \mathrm{CDCl}_{3}\right): \delta 171.7$, 171.7, 163.0, 162.8, 162.7, 155.0, 154.9, 152.3, 152.1, 151.7, 151.4, 144.6, 144.5, 141.8, 141.7, 135.7, 135.6, 129.4, 129.4, 128.8, 127.3, 127.3, 112.8, 111.5, 110.1, 95.8, 95.7, 94.3, 94.2, 94.0, 61.3, 61.2, 55.1, 55.1, 52.9, 52.8, 52.7, 52.5, 52.5, 38.3, 38.2. HRMS (ESI-TOF) m/z: [M + $\mathrm{Na}]^{+}$Calcd for $\mathrm{C}_{23} \mathrm{H}_{23} \mathrm{~F}_{2} \mathrm{~N}_{1} \mathrm{O}_{9} \mathrm{Na}^{+}$518.1233; Found 518.1239.

Oseltamivir conjugates - general procedure: A solution of oseltamivir phosphate (1 equiv) and triethylamine (2.5 equiv) in dichloromethane was cooled to $-20^{\circ} \mathrm{C}$. Then a solution of OND Nhydroxysuccinimidyl carbonate (1.1 to 1.2 equiv) in dichloromethane was added dropwise and

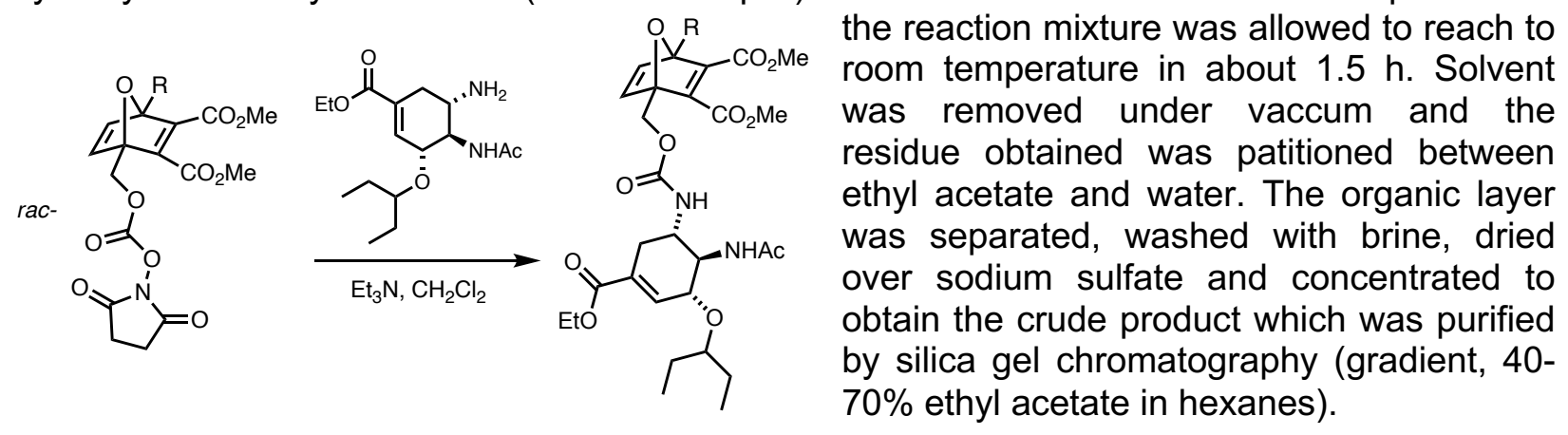

Dimethyl-1-((((1S,5R,6R)-6-acetamido-3-(ethoxycarbonyl)-5-(pentan-3-yloxy)cyclohex-3en-1-yl)carbamoyl)oxy)methyl)-4-(6-(trifluoromethyl)pyridin-3-yl)-7oxabicyclo[2.2.1]hepta-2,5-diene-2,3-dicarboxylate, 1n

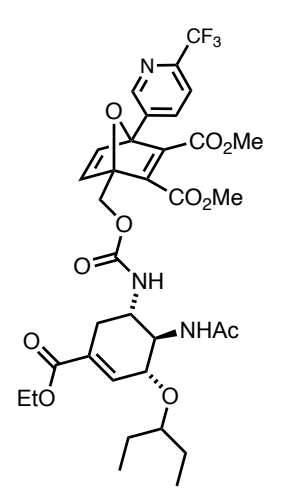

Pale yellow solid; yield $65 \%$ (14 mg, mixture of diastereomers); ${ }^{1} \mathrm{H}$ NMR $(400$ $\left.\mathrm{MHz}, \mathrm{CDCl}_{3}\right): \delta 8.89(\mathrm{br} \mathrm{s}, 1 \mathrm{H}), 8.00-8.10(\mathrm{~m}, 1 \mathrm{H}), 7.74(\mathrm{~d}, \mathrm{~J}=8.2 \mathrm{~Hz}, 1 \mathrm{H})$, $7.44(\mathrm{dd}, \mathrm{J}=1.6,4.8 \mathrm{~Hz}, 1 \mathrm{H}), 7.20(\mathrm{dd}, \mathrm{J}=1.0,5.0 \mathrm{~Hz}, 1 \mathrm{H}), 6.80(\mathrm{br} \mathrm{s}, 1 \mathrm{H})$, 5.54-5.71 (m, 2H), 4.92, 4.88 (ABq, J = 12.6Hz, 2H), 4.15-4.26 (m, 2H), 4.05$4.14(\mathrm{~m}, 1 \mathrm{H}), 3.94-4.04(\mathrm{~m}, 1 \mathrm{H}), 3.86-3.94(\mathrm{~m}, 1 \mathrm{H}), 3.83,3.82(2 \mathrm{~s}, 3 \mathrm{H}), 3.67$ (s, 3H), 3.34-3.42 (m, 1H), $2.75(2 \mathrm{dt}, \mathrm{J}=5.1 \mathrm{~Hz}, 1 \mathrm{H}), 2.34-2.41(\mathrm{~m}, 1 \mathrm{H}), 1.95$ (s, 3H), 1.44-1.58 (m, 4H), $1.29(\mathrm{td}, \mathrm{J}=1.0,6.9 \mathrm{~Hz}, 3 \mathrm{H}), 0.83-0.92(\mathrm{~m}, 6 \mathrm{H})$. ${ }^{13} \mathrm{C} \mathrm{NMR}\left(125 \mathrm{MHz}, \mathrm{CDCl}_{3}\right): \delta 171.0,170.9,165.9,163.6,163.5,162.8,156.0$, 156.0, 155.8, 155.8, 150.4, 150.3, 148.5, 144.6, 144.6, 144.5, 144.4, 136.8, $136.7,136.35,136.29,132.88,129.39,122.41,120.16,95.00,94.98,94.16$, 
82.07, 82.0, 75.1, 74.9, 61.2, 61.1, 61.0, 53.3, 53.1, 52.7, 52.6, 52.5, 49.7, 49.6, 30.4, 30.3, 26.1, 26.1, 25.7, 23.3, 23.3, 14.1, 9.4, 9.3, 9.3. HRMS (ESI-TOF) $\mathrm{m} / \mathrm{z}$ : $[\mathrm{M}+\mathrm{H}]^{+}$Calcd for $\mathrm{C}_{34} \mathrm{H}_{41} \mathrm{~F}_{3} \mathrm{~N}_{3} \mathrm{O}_{11} \mathrm{H}^{+}$724.2688; Found 724.2684.

Dimethyl-1-(((((1S,5R,6R)-6-acetamido-3-(ethoxycarbonyl)-5-(pentan-3-yloxy)cyclohex-3en-1-yl)carbamoyl)oxy)methyl)-4-(3,5-bis(trifluoromethyl)phenyl)-7-

oxabicyclo[2.2.1] hepta-2,5-diene-2,3-dicarboxylate, 10

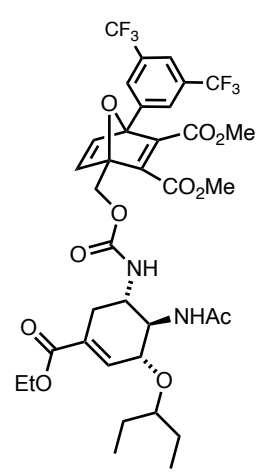

White solid; yield $60 \%$ (17 mg, mixture of diastereomers); ${ }^{1} \mathrm{H}$ NMR $(400 \mathrm{MHz}$ $\left.\mathrm{CDCl}_{3}\right): \delta 7.99(\mathrm{br} \mathrm{s}, 2 \mathrm{H}), 7.91(\mathrm{br} \mathrm{s}, 1 \mathrm{H}), 7.42(\mathrm{~d}, \mathrm{~J}=5.2 \mathrm{~Hz}, 1 \mathrm{H}), 7.21(\mathrm{dd}, \mathrm{J}=$ 1.2, $5.2 \mathrm{~Hz}), 6.81$ (br s, $1 \mathrm{H}), 5.48-5.73(\mathrm{~m}, 2 \mathrm{H}), 4.93,4.89(\mathrm{ABq}, \mathrm{J}=13.0 \mathrm{~Hz}$, $2 \mathrm{H}), 4.21(\mathrm{q}, \mathrm{J}=7.1 \mathrm{~Hz}, 2 \mathrm{H}), 4.06-4.15(\mathrm{~m}, 1 \mathrm{H}), 3.97-4.05(\mathrm{~m}, 1 \mathrm{H}), 3.86-3.96$ $(\mathrm{m}, 1 \mathrm{H}), 3.83,3.81(2 \mathrm{~s}, 3 \mathrm{H}), 3.65(\mathrm{~s}, 3 \mathrm{H}), 3.34-3.40(\mathrm{~m}, 1 \mathrm{H}), 2.78,2.73(2 \mathrm{t}, \mathrm{J}=$ $6.2 \mathrm{~Hz}, 1 \mathrm{H}), 2.33-2.44(\mathrm{~m}, 1 \mathrm{H}), 1.97(2 \mathrm{~s}, 3 \mathrm{H}), 1.45-1.56(\mathrm{~m}, 4 \mathrm{H}), 1.23-1.32(\mathrm{~m}$, $3 \mathrm{H}), 0.84-0.92(\mathrm{~m}, 6 \mathrm{H}) .{ }^{13} \mathrm{C}$ NMR $\left(125 \mathrm{MHz} \mathrm{CDCl}_{3}\right): \delta 170.9,170.9,165.9$, 163.63, 162.7, 156.6, 155.7, 144.7, 144.5, 144.6, 136.9, 136.7, 136.4, 132.0, $131.8,129.4,127.3,124.1,122.8,122.0,95.2,94.9,82.1,82.0,75.1,74.9$, 61.3, 61.2, 61.0, 53.3, 53.1, 52.7, 52.6, 52.4, 49.6, 49.5, 30.5, 30.3, 29.7, 26.1, 25.7, 23.3, 14.2, 9.4, 9.3, 9.2. HRMS (ESI-TOF) $\mathrm{m} / \mathrm{z}:[\mathrm{M}+\mathrm{H}]^{+}$Calcd for $\mathrm{C}_{36} \mathrm{H}_{41} \mathrm{~F}_{6} \mathrm{~N}_{2} \mathrm{O}_{11} \mathrm{H}^{+}$791.2609; Found 791.2606.

Benzyl 5-nitrofuran-2-carboxylate ${ }^{12}$ (S33) and benzyl 5-fluorofuran-2-carboxylate ${ }^{13}$ (S34): Prepared according to literature procedures; the NMR data agreed with the

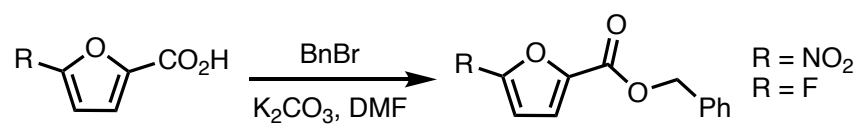
reported spectra.

tert-butyl ((5-(trifluoromethyl)furan-2yl)methyl)carbamate (S35): The furan (0.63 mmol) was dissolved in DCM (3 mL).

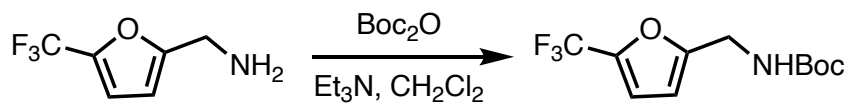

Triethyl amine ( 1 equiv) was added, followed by di-tert-butyl dicarbonate ( $\mathrm{Boc}_{2} \mathrm{O}, 1.1$ equiv). The reaction was stirred at room temperature overnight. Water and ethyl acetate were added. The organic layer was washed with water and $0.5 \mathrm{M} \mathrm{HCl}$, dried over sodium sulfate, filtered and concentrated under vacuum. White solid. $82 \%$ yield $(155 \mathrm{mg})$.

${ }^{1} \mathrm{H}$ NMR $\left(500 \mathrm{MHz}, \mathrm{CDCl}_{3}\right): \delta 6.71(\mathrm{~m}, 1 \mathrm{H}), 6.29$ (s, 1H), 4.94 (br s, 1H), 4.34- 4.33 (s, 2H), 1.45 (s, 9H). ${ }^{13} \mathrm{C}$ NMR (125 MHz, $\left.\mathrm{CDCl}_{3}\right): \delta$ 155.4, 155.1, 141.7- 140.6, 120.0, 117.9, 112.4, 107.7, 80.1, 37.5, 28.3. HRMS (ESI-TOF) $\mathrm{m} / \mathrm{z}$ : $[\mathrm{M}+\mathrm{Na}]^{+}$Calcd for $\mathrm{C}_{11} \mathrm{H}_{14} \mathrm{~F}_{3} \mathrm{NO}_{3} \mathrm{Na}^{+}$288.0823; Found 288.0825 .

Dimethyl-1-((4-nitrobenzamido)methyl)-4-(trifluoromethyl)-7-oxabicyclo[2.2.1]hepta-2,5diene-2,3-dicarboxylate, $1 \mathrm{~m}$ : The OND $(0.12 \mathrm{mmol})$ was dissolved in DCM $(0.7 \mathrm{~mL})$. p-toluenesulfonic acid ( $\mathrm{TsOH}, 1.5$ equiv) was added and the reaction was stirred at room temperature overnight. Triethyl amine (5 equiv) and 4-nitrobenzoyl

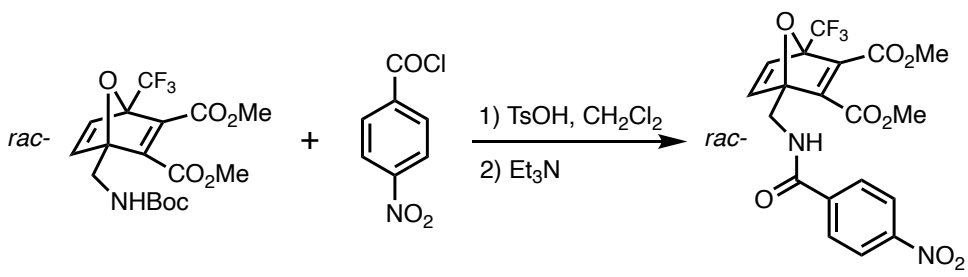
chloride (1.1 equiv) were added. The reaction was stirred at room temperature for 16 hours. The organic layer was washed with sat. $\mathrm{NaHCO}_{3}$ (x 2), dried over sodium sulfate, filtered and concentrated under vacuum. The residue obtained was purified by silica gel chromatography (50\% ethyl acetate in hexanes) to yield product as a yellow oil (21 mg, $38 \%$ yield). 
${ }^{1} \mathrm{H}$ NMR $\left(700 \mathrm{MHz}, \mathrm{CDCl}_{3}\right): \delta 8.31(\mathrm{~d}, \mathrm{~J}=8.8 \mathrm{~Hz}, 2 \mathrm{H}), 7.94(\mathrm{~d}, \mathrm{~J}=8.8 \mathrm{~Hz}, 2 \mathrm{H}), 7.21-7.24(\mathrm{~m}$, $2 \mathrm{H}), 6.90(\mathrm{t}, \mathrm{J}=5.7 \mathrm{~Hz}, 1 \mathrm{H}), 4.48(\mathrm{dd}, \mathrm{J}=6.0,15.0 \mathrm{~Hz}, 1 \mathrm{H}), 4.36(\mathrm{dd}, \mathrm{J}=6.0,15.0 \mathrm{~Hz}, 1 \mathrm{H})$, $3.86(\mathrm{~s}, 3 \mathrm{H}), 3.83(\mathrm{~s}, 3 \mathrm{H}) .{ }^{13} \mathrm{C}$ NMR $\left(176 \mathrm{MHz}, \mathrm{CDCl}_{3}\right): \delta 165.5,162.43,162.3,153.2,150.3$, 149.8, 145.7, 140.9, 139.3, 128.3, 123.9, 96.4, 53.0, 53.0, 38.3. HRMS (ESI-TOF) m/z: [M + H] ${ }^{+}$ Calcd for $\mathrm{C}_{19} \mathrm{H}_{16} \mathrm{~F}_{3} \mathrm{~N}_{2} \mathrm{O}_{8} \mathrm{H}^{+}$457.0854; Found 457.0851.

3-(5-methylfuran-2-yl)oxetan-3-ol, S36: This compound was prepared according to a literature producure. ${ }^{14}$ The NMR data agreed with the reported spectrum of this compound.

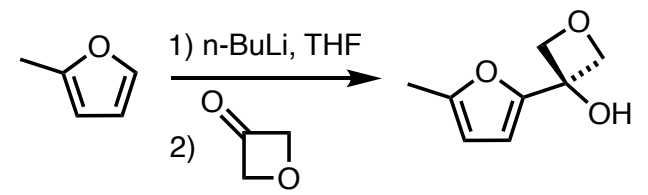

5-Substituted furan carboxaldehydes - general procedure: 5-bromofuran-2-carbaldehyde (1

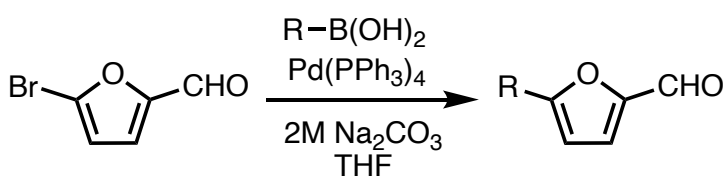
equiv), boronic acid (1.05 equiv), 2M sodium carbonate (5 equiv) and Tetrakis(triphenylphosphine)palladium(0) (0.035 equiv) were added to a vial. The solvent, THF, was added and the solution was heated at $80^{\circ} \mathrm{C}$ (oil bath) overnight. The reaction was then cooled to room temperature and concentrated under vacuum. Water and ethyl acetate were added. The organic layer was extracted twice with ethyl acetate, washed with brine, dried over sodium sulfate, filtered and concentrated under vacuum. The residue obtained was purified by silica gel chromatography ( $15 \%$ ethyl acetate in hexanes).

\section{5-(3,5-bis(trifluoromethyl)phenyl)furan-2-carbaldehyde, S37}

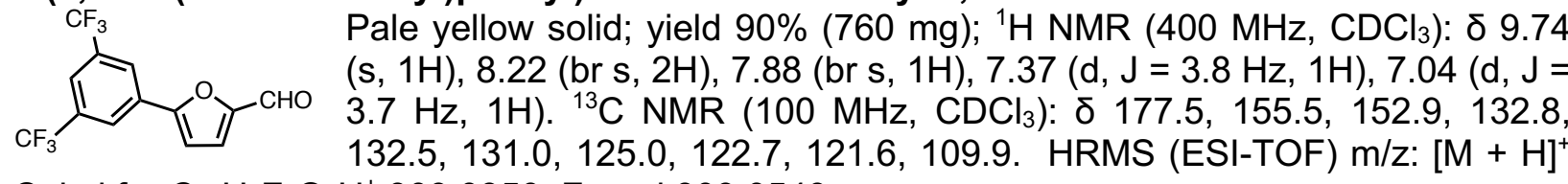
Calcd for $\mathrm{C}_{13} \mathrm{H}_{6} \mathrm{~F}_{6} \mathrm{O}_{2} \mathrm{H}^{+}$309.0350; Found 309.0549.

\section{5-(6-(trifluoromethyl)pyridin-3-yl)furan-2-carbaldehyde, $\mathbf{S 3 8}$}

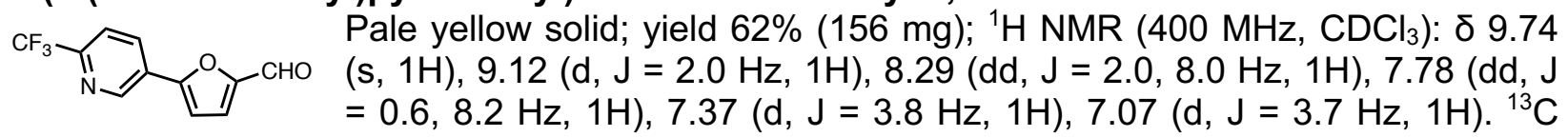
NMR $\left(100 \mathrm{MHz}, \mathrm{CDCl}_{3}\right): \delta 177.5,154.4,153.2,146.5,133.3,127.7,122.5,120.7,120.7,119.9$, 110.3. HRMS (ESI-TOF) $\mathrm{m} / \mathrm{z}$ : $[\mathrm{M}+\mathrm{H}]^{+}$Calcd for $\mathrm{C}_{11} \mathrm{H}_{6} \mathrm{~F}_{3} \mathrm{~N}_{1} \mathrm{O}_{2} \mathrm{H}^{+}$242.0429; Found 242.0435.

Furan carboxyaldehyde reduction - general procedure: Lithium borohydride (1 equiv) was dissolved in THF and cooled to $0^{\circ} \mathrm{C}$. The furan $(1$

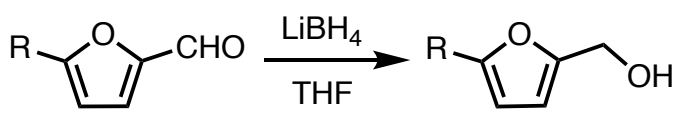
equiv) was added dropwise at $0^{\circ} \mathrm{C}$. The reaction was stirred and allowed to reach room temperature over 18 hours. Water was added to quench the reaction. The organic layer was extracted with DCM, washed with brine, dried over sodium sulfate, filtered and concentrated under vacuum.

\section{(5-(3,5-bis(trifluoromethyl)phenyl)furan-2-yl)methanol, S39}

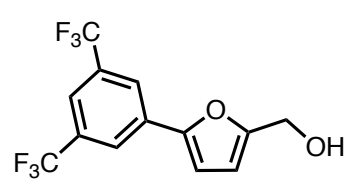

Pale yellow solid; yield 74\% (92 mg); ${ }^{1} \mathrm{H}$ NMR (400 MHz, $\mathrm{CDCl}_{3}$ ): $\delta 8.06$ (br s, 2H), 7.74 (br s, 1H), $6.81(\mathrm{~d}, \mathrm{~J}=3.3 \mathrm{~Hz}, 1 \mathrm{H}), 6.46(\mathrm{~d}, \mathrm{~J}=3.3 \mathrm{~Hz}$, $1 \mathrm{H}), 4.72(\mathrm{~d}, \mathrm{~J}=6.1 \mathrm{~Hz}, 2 \mathrm{H}), 1.80(\mathrm{t}, \mathrm{J}=6.1 \mathrm{~Hz}, 1 \mathrm{H}) .{ }^{13} \mathrm{C} \mathrm{NMR}(125 \mathrm{MHz}$, $\left.\mathrm{CDCl}_{3}\right): \delta 155.4,151.0,132.5,132.4,132.1,124.3,123.5,122.2,120.5$, 110.3, 108.6, 57.6. HRMS (ESI-TOF) $\mathrm{m} / \mathrm{z}:[\mathrm{M}+\mathrm{H}]^{+}$Calcd for $\mathrm{C}_{13} \mathrm{H}_{8} \mathrm{~F}_{6} \mathrm{O}_{2} \mathrm{H}^{+}$311.0507; Found 311.0514. 


\section{(5-(6-(trifluoromethyl)pyridin-3-yl)furan-2-yl)methanol, S40}

Pale yellow solid; yield 44\% $(40 \mathrm{mg}) ;{ }^{1} \mathrm{H} \mathrm{NMR}\left(500 \mathrm{MHz}, \mathrm{CDCl}_{3}\right): \delta 8.98$
$(\mathrm{~d}, \mathrm{~J}=1.9 \mathrm{~Hz}, 1 \mathrm{H}), 8.09-8.07(\mathrm{dd}, \mathrm{J}=1.6,8.2 \mathrm{~Hz}, 1 \mathrm{H}), 7.69-7.68(\mathrm{~d}, \mathrm{~J}$ $1 \mathrm{H}), 4.71$ (s, 2H), 1.88 (br s, 1H). ${ }^{13} \mathrm{C}$ NMR (125 MHz, $\left.\mathrm{CDCl}_{3}\right): \delta 155.8,149.5,146.0,145.2$, 131.4, 129.1, 120.6, 120.5 -120.5, 110.3, 109.2, 57.5. HRMS (ESI-TOF) m/z: [M + H] $]^{+}$Calcd for $\mathrm{C}_{11} \mathrm{H}_{8} \mathrm{~F}_{3} \mathrm{~N}_{1} \mathrm{O}_{2} \mathrm{H}^{+}$244.0585; Found 244.0579.

\section{(5-cyclopropylfuran-2-yl)methanol, S41}

Yellow oil; yield 100\% (44 mg); ${ }^{1} \mathrm{H} \mathrm{NMR}\left(500 \mathrm{MHz}, \mathrm{CDCl}_{3}\right)$ : $\delta$ 6.17- $6.16(\mathrm{~d}, \mathrm{~J}=$ $3.1 \mathrm{~Hz}, 1 \mathrm{H}), 5.89-5.88(\mathrm{~d}, \mathrm{~J}=3.1 \mathrm{~Hz}, 1 \mathrm{H}), 4.53(\mathrm{~s}, 2 \mathrm{H}), 1.90-1.85(\mathrm{~m}, 1 \mathrm{H})$, $1.25(\mathrm{br} \mathrm{s}, 1 \mathrm{H}), 0.89-0.85(\mathrm{~m}, 2 \mathrm{H}), 0.78-0.76(\mathrm{~m}, 2 \mathrm{H}) .{ }^{13} \mathrm{C}$ NMR $(125 \mathrm{MHz}$, $\left.\mathrm{CDCl}_{3}\right): \delta$ 157.8, 151.8, 108.7, 104.1, 57.6, 8.8, 6.6. HRMS (ESI-TOF) m/z: $[\mathrm{M}+\mathrm{H}]^{+}$Calcd for $\mathrm{C}_{8} \mathrm{H}_{10} \mathrm{O}_{2} \mathrm{H}^{+}$139.0759; Found 139.0756 .

\section{(5-fluorofuran-2-yl)methanol, S42}<smiles>OCc1ccc(F)o1</smiles>

Reaction used 9 equiv of lithium borohydride, yellow oil; yield $65 \%$ (16 mg); The product is volatile and so the crude mixture was used for the next step without purification. The NMR signals of desired product were suppressed by excess benzyl alcohol. HRMS (ESI-TOF) m/z: $[2 \mathrm{M}+\mathrm{H}]^{+}$Calcd for $\mathrm{C}_{10} \mathrm{H}_{10} \mathrm{~F}_{2} \mathrm{O}_{4} \mathrm{H}^{+}$233.0625; Found 233.0627.

5-cyclopropylfuran-2-carbaldehyde, S43: 5bromofuran-2-carbaldehyde (1) equiv), cyclopropylboronic acid (2 equiv), cesium carbonate (3 equiv), di(1-adamantyl)-n-butylphosphine (3\% mol) and Palladium(II) acetate $(2 \% \mathrm{~mol})$ were added to a

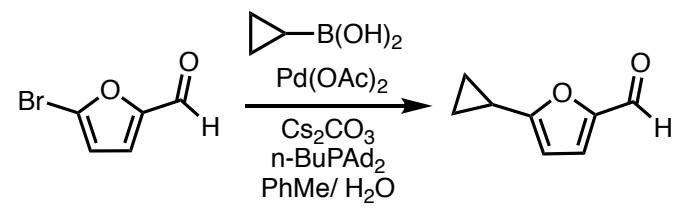
vial. Toluene and water $(10: 1)$ were added and the solution was heated at $100^{\circ} \mathrm{C}$ (oil bath) overnight. The reaction was then cooled to room temperature. Water and $\mathrm{CH}_{2} \mathrm{Cl}_{2}$ were added. The organic layer was extracted three times with DCM, dried over sodium sulfate, filtered and concentrated under vacuum. The residue obtained was purified by silica gel chromatography (10\% ethyl acetate in hexanes). Yellow oil. $74 \%$ yield $(40 \mathrm{mg})$.

${ }^{1} \mathrm{H}$ NMR $\left(500 \mathrm{MHz}, \mathrm{CDCl}_{3}\right): \delta 9.41(\mathrm{~s}, 1 \mathrm{H}), 7.13(\mathrm{~d}, \mathrm{~J}=3.6 \mathrm{~Hz}, 1 \mathrm{H}), 6.16(\mathrm{~d}, \mathrm{~J}=3.6 \mathrm{~Hz}, 1 \mathrm{H})$, 1.94-2.01 (m, 1H), 0.93-1.05 (m, 4H). ${ }^{13} \mathrm{C}$ NMR (125 MHz, $\left.\mathrm{CDCl}_{3}\right): \delta 176.2,165.1,151.1,106.8$, 9.5, 8.4. HRMS (ESI-TOF) m/z: [M + H] $]^{+}$Calcd for $\mathrm{C}_{8} \mathrm{H}_{8} \mathrm{O}_{2} \mathrm{H}^{+} 137.0603$; Found 137.0601 .

(5-(difluoromethyl)furan-2-yl)methyl acetate,

S44: This compound was prepared according to a literature producure. ${ }^{15}$ The NMR data agreed with the reported spectrum of this compound.

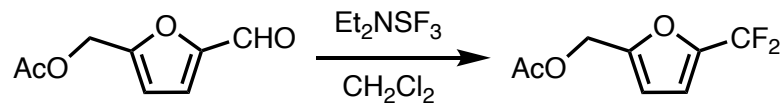

(5-(difluoromethyl)furan-2-yl)methanol, S45: This compound was prepared according to a literature producure. ${ }^{15}$ The NMR data agreed with the reported spectrum of this compound.

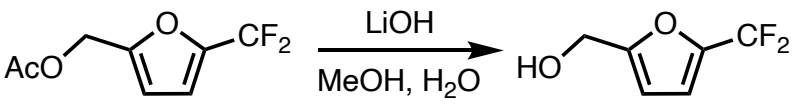




\section{REFERENCES}

1. Frisch, M.; Trucks, G.; Schlegel, H.; Scuseria, G.; Robb, M.; Cheeseman, J.; Scalmani, G.; Barone, V.; Mennucci, B.; Petersson, G., Gaussian 16 Revision A. 03, 2016. Gaussian Inc. Wallingford CT 2009.

2. Zhao, Y.; Truhlar, D. G., The M06 suite of density functionals for main group thermochemistry, thermochemical kinetics, noncovalent interactions, excited states, and transition elements: two new functionals and systematic testing of four M06-class functionals and 12 other functionals. Theor. Chem. Accounts 2007, 120, 215-241.

3. Grimme, S.; Antony, J.; Ehrlich, S.; Krieg, H., A consistent and accurate ab initio parametrization of density functional dispersion correction (DFT-D) for the 94 elements H-Pu. J. Chem. Phys. 2010, 132, 154104.

4. Goerigk, L.; Hansen, A.; Bauer, C.; Ehrlich, S.; Najibi, A.; Grimme, S., A look at the density functional theory zoo with the advanced GMTKN55 database for general main group thermochemistry, kinetics and noncovalent interactions. Phys. Chem. Chem. Phys. 2017, 19, 32184-32215.

5. Funes-Ardoiz, I.; Paton, R. GoodVibes: GoodVibes v1.0.0, 2006.

6. Basarab, G. S.; Hill, P.; Eyermann, C. J.; Gowravaram, M.; Kack, H.; Osimoni, E., Design of inhibitors of Helicobacter pylori glutamate racemase as selective antibacterial agents: incorporation of imidazoles onto a core pyrazolopyrimidinedione scaffold to improve bioavailabilty. Bioorg. Med. Chem. Lett. 2012, 22, 5600-5607.

7. Ouairy, C.; Michel, P.; Delpech, B.; Crich, D.; Marazano, C., Synthesis of N-acyl-5-aminopenta-2,4dienals via base-induced ring-opening of $\mathrm{N}$-acylated furfurylamines: scope and limitations. J. Org. Chem. 2010, 75, 4311-4314.

8. Coombs, T. C.; Lee, M. D. t.; Wong, H.; Armstrong, M.; Cheng, B.; Chen, W.; Moretto, A. F.; Liebeskind, L. S., Practical, scalable, high-throughput approaches to eta3-pyranyl and eta3-pyridinyl organometallic enantiomeric scaffolds using the Achmatowicz reaction. J. Org. Chem. 2008, 73, 882888.

9. White, A. R.; Kozlowski, R. A.; Tsai, S.-C.; Vanderwal, C. D., A Direct Synthesis of Highly Substituted m-Rich Aromatic Heterocycles from Oxetanes. Angew. Chem. Int. Ed. 2017, 56, 10525-10529.

10. Belmessieri, D.; Cordes, D. B.; Slawin, A. M.; Smith, A. D., Telescoped synthesis of stereodefined pyrrolidines. Org. Lett. 2013, 15, 3472-3475.

11. Massari, S.; Desantis, J.; Nannetti, G.; Sabatini, S.; Tortorella, S.; Goracci, L.; Cecchetti, V.; Loregian, A.; Tabarrini, O., Efficient and regioselective one-step synthesis of 7-aryl-5-methyl- and 5-aryl-7methyl-2-amino-[1,2,4]triazolo[1,5-a]pyrimidine derivatives. Org. Biomol. Chem. 2017, 15, 7944-7955.

12. Yanagita, H.; Fudo, S.; Urano, E.; Ichikawa, R.; Ogata, M.; Yokota, M.; Murakami, T.; Wu, H.; Chiba, J.; Komano, J.; Hoshino, T., Structural modulation study of inhibitory compounds for ribonuclease $\mathrm{H}$ activity of human immunodeficiency virus type 1 reverse transcriptase. Chem. Pharm. Bull. 2012, 60, 764-771.

13. Song, R.; Lin, W.; Jiang, Q., A convenient synthesis of 5-fluorofuran-2-carboxylic acid. Tetrahedron Lett. 2011, 52, 4965-4966.

14. Croft, R. A.; Mousseau, J. J.; Choi, C.; Bull, J. A., Lithium-Catalyzed Thiol Alkylation with Tertiary and Secondary Alcohols: Synthesis of 3-Sulfanyl-Oxetanes as Bioisosteres. Chemistry 2018, 24, 818-821.

15. Duffey, M. O. E., Dylan B.; Hu, Zhigen; Ito, Mitsuhiro; Langston, Steven P.; Mcintyre, Charles; Mizutani, Hirotake; Xu, He Preparation of heteroaryl sulfamate compounds useful as inhibitors of Sumo Activating Enzyme (SAE). January 8, 2015. 
D. NMR SPECTRA

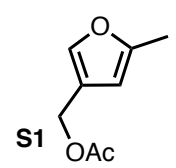

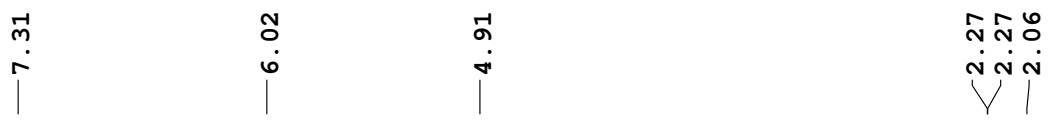

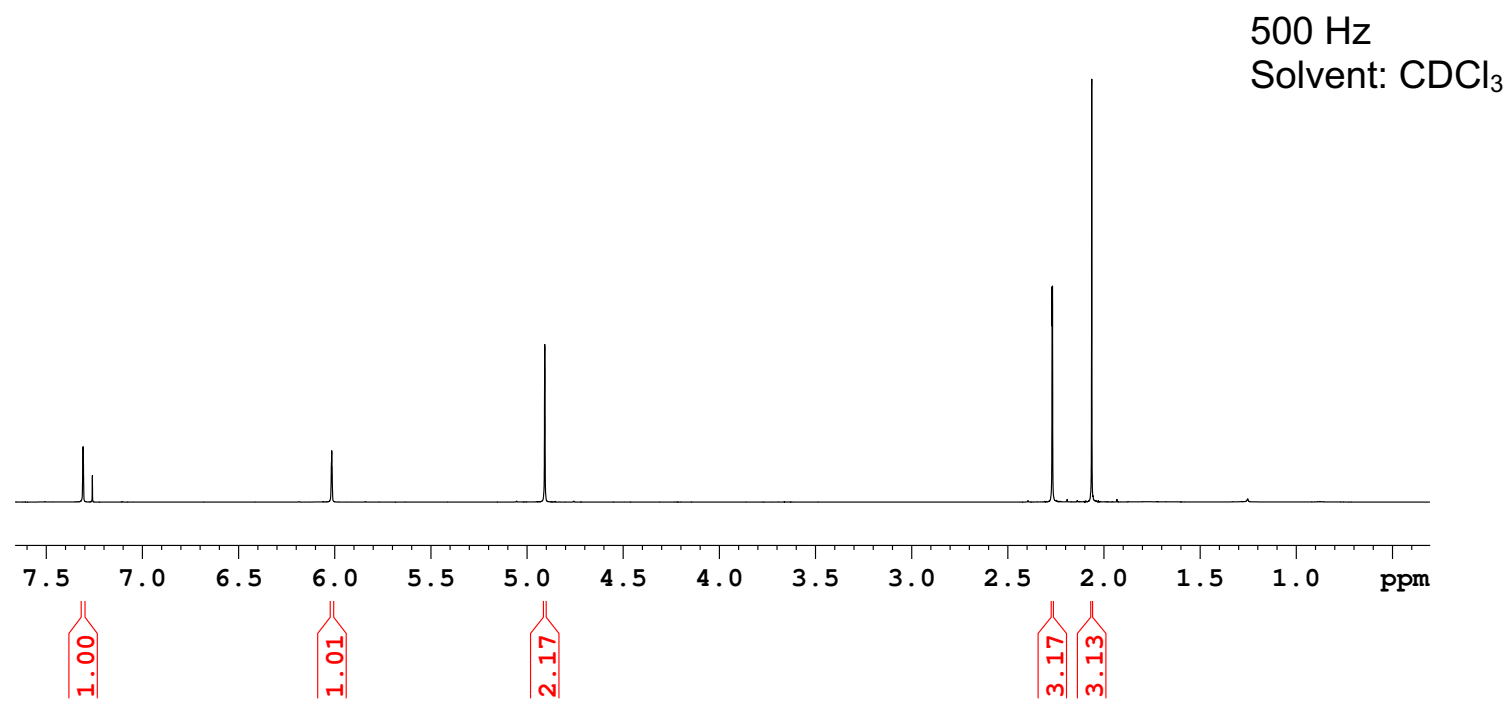




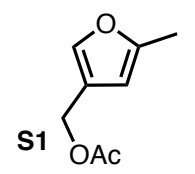

furan-2me-4-oac

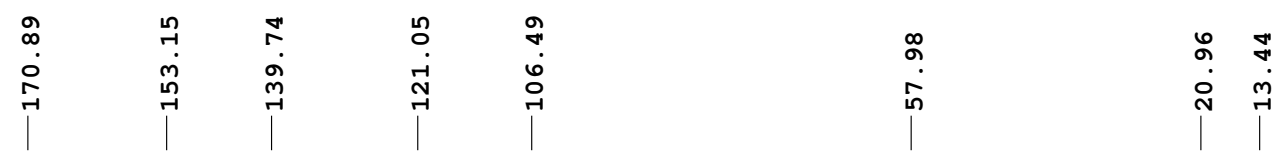

$125 \mathrm{~Hz}$

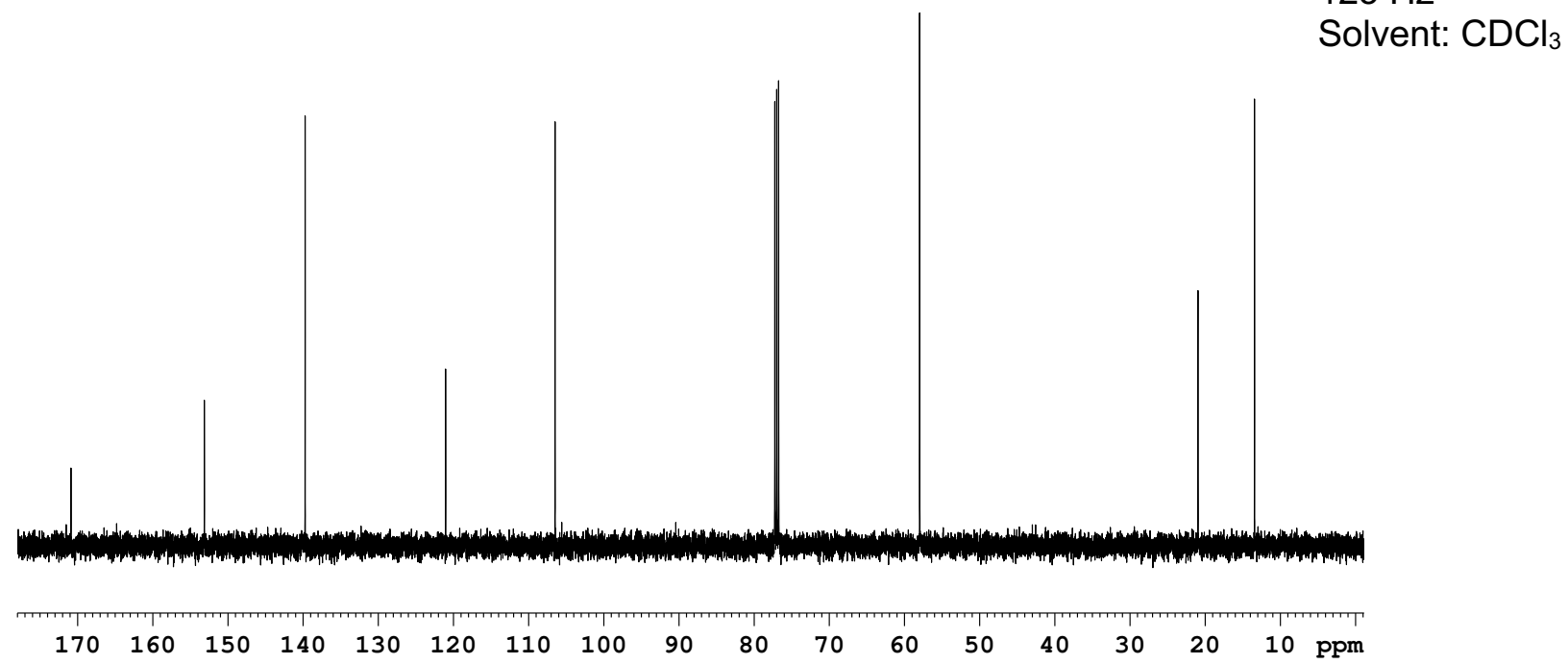


<smiles>CC(=O)OCc1coc(-c2ccccc2)c1</smiles>

fur $-4 \circ a c-2 p h$

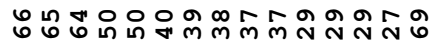
ristitititititiog

s.

욱

(1)

เก

N

$500 \mathrm{~Hz}$

Solvent: $\mathrm{CDCl}_{3}$

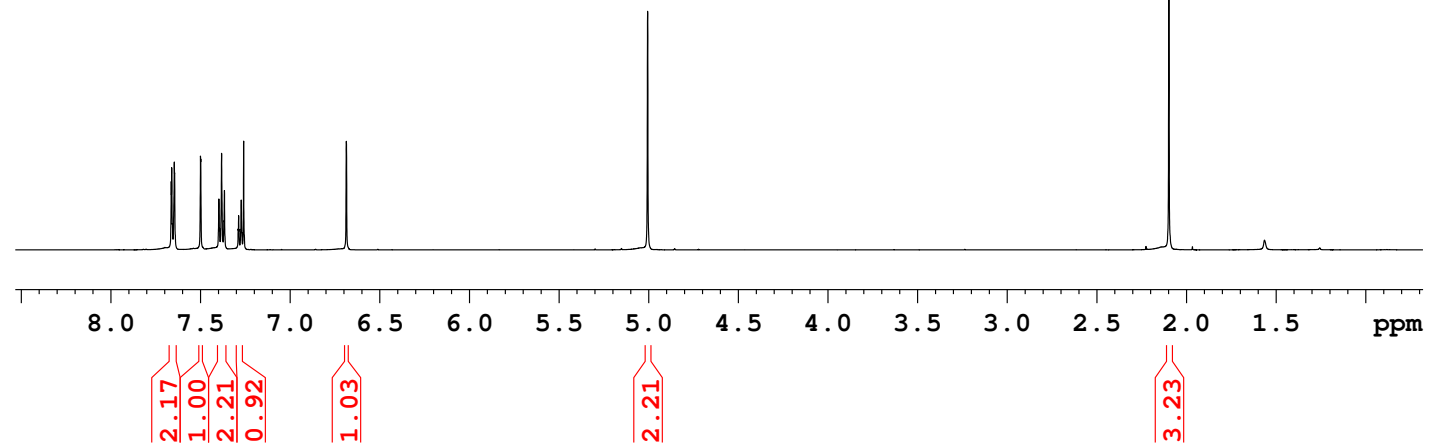


<smiles>CC(=O)OCc1coc(-c2ccccc2)c1</smiles>

4-oac-2-ph furan
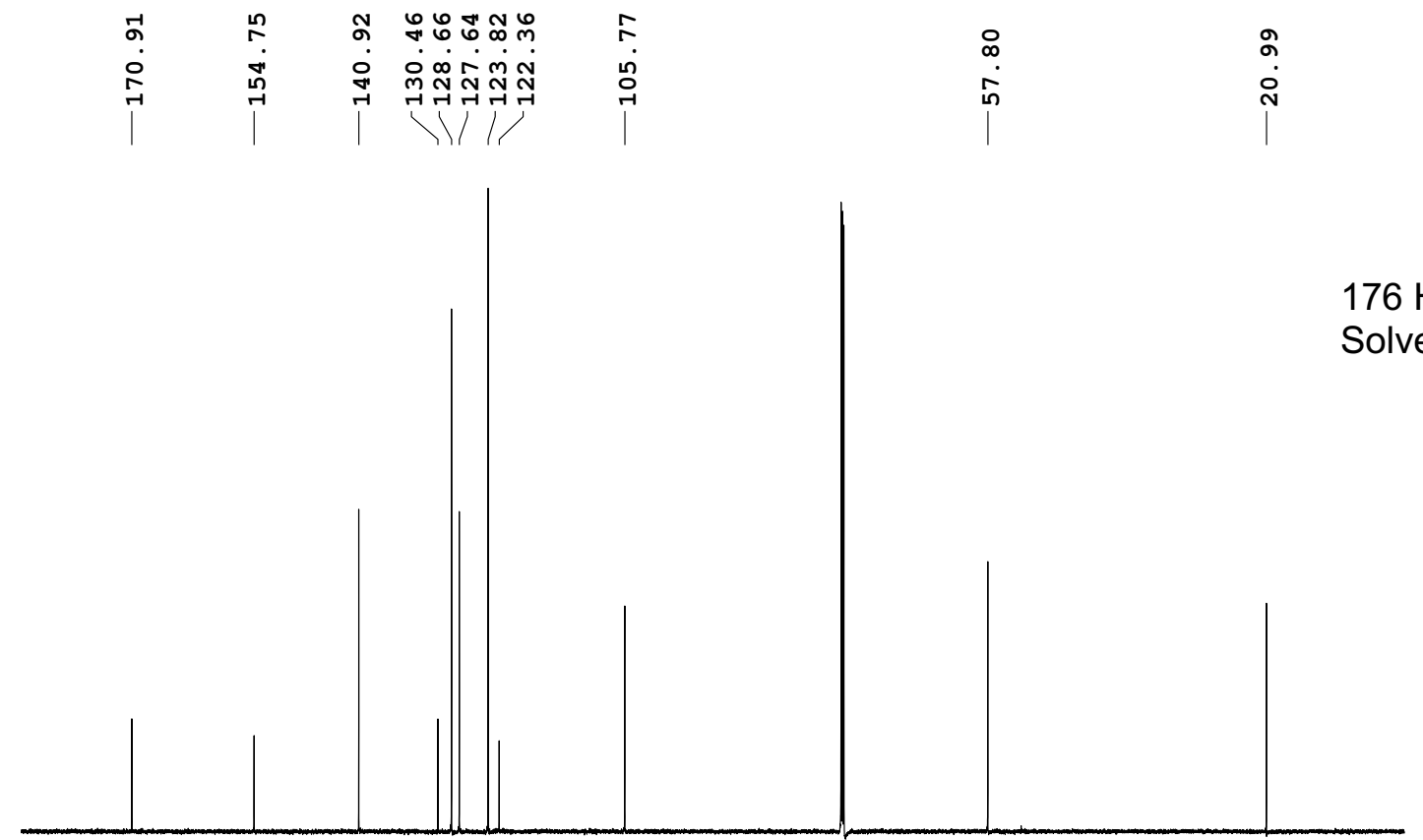

$176 \mathrm{~Hz}$

Solvent: $\mathrm{CDCl}_{3}$

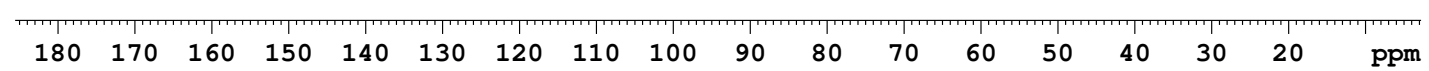


<smiles>COc1ccc(-c2cc(COC(C)=O)co2)cc1</smiles>

furan-2ph-ome-4oac
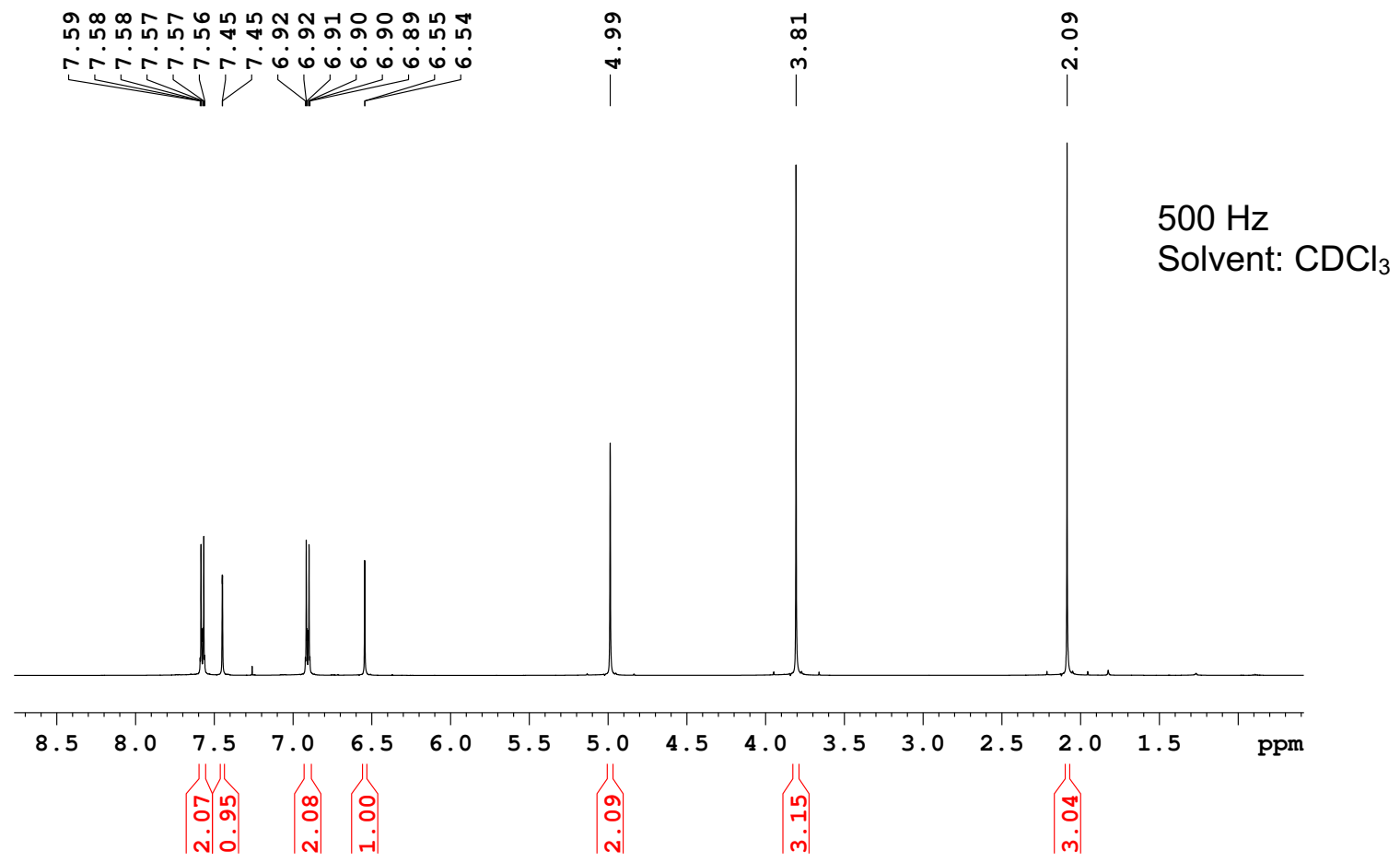
<smiles>COc1ccc(-c2cc(COC(C)=O)co2)cc1</smiles>

furan-2ph-ome-4oac
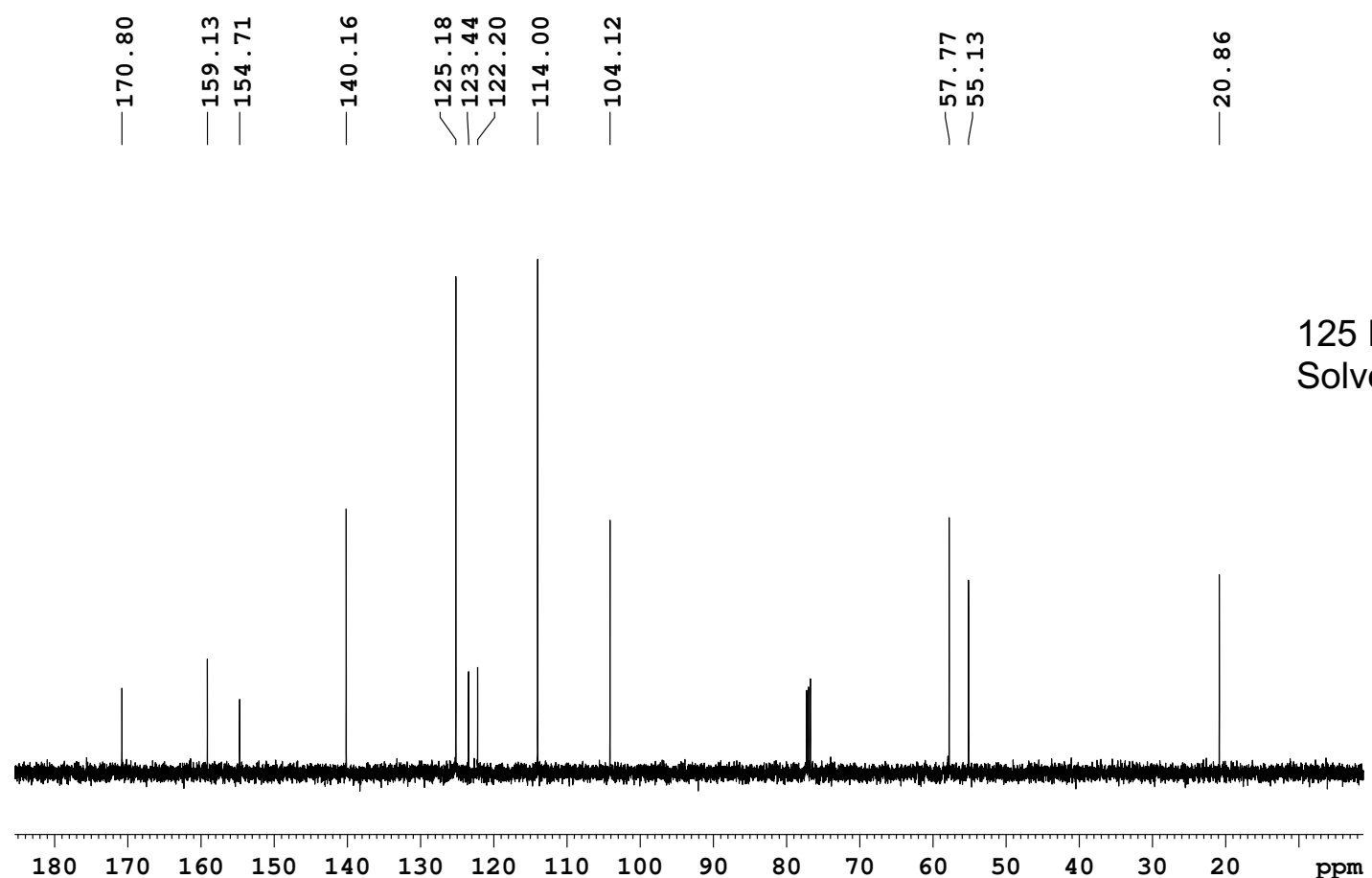
<smiles>O=C([O-])OC([SiH3])c1coc(-c2ccc([N+](=O)[O-])cc2)c1</smiles>

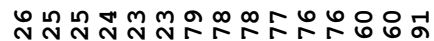

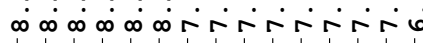

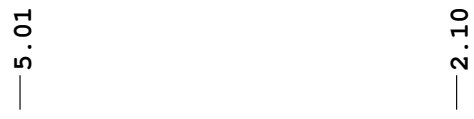

$500 \mathrm{~Hz}$

Solvent: $\mathrm{CDCl}_{3}$

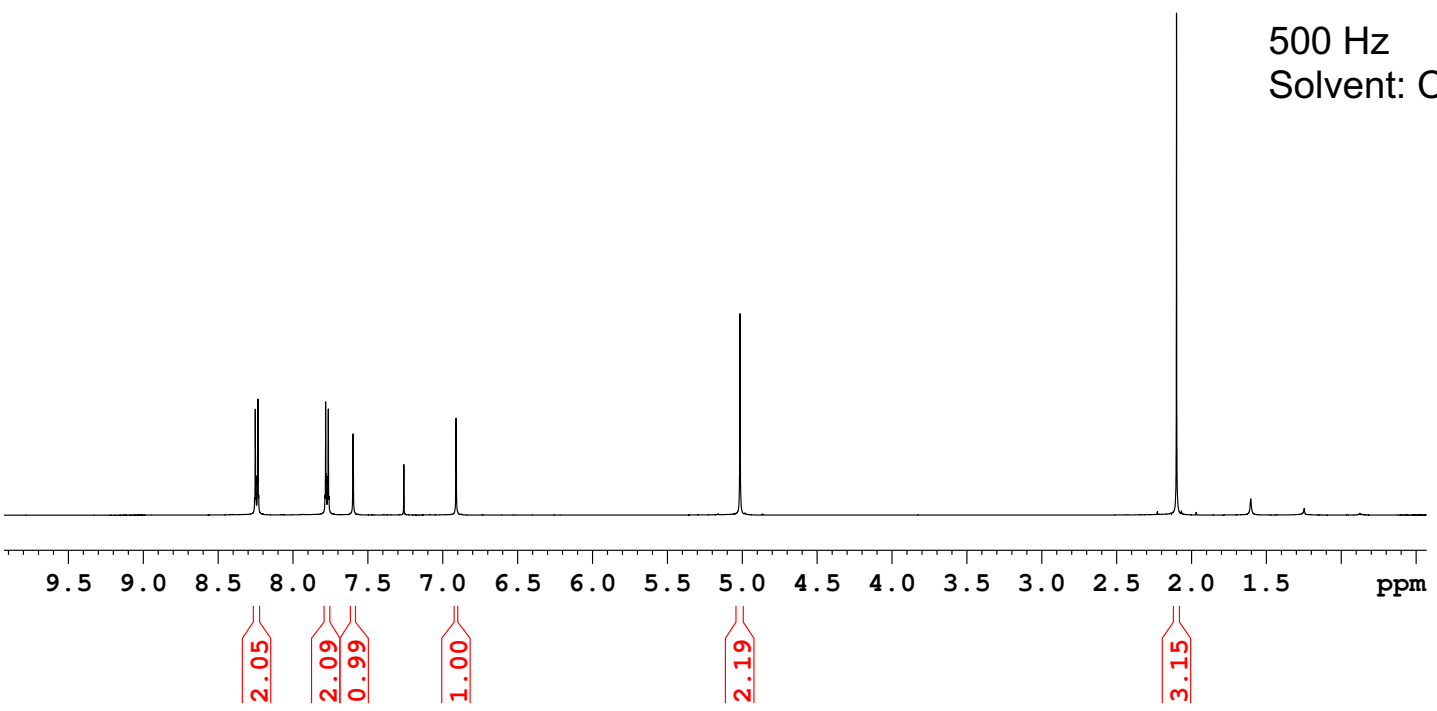


<smiles>O=[N+]([O-])c1ccc(-c2cc(C([Si])O[Na])co2)cc1</smiles>

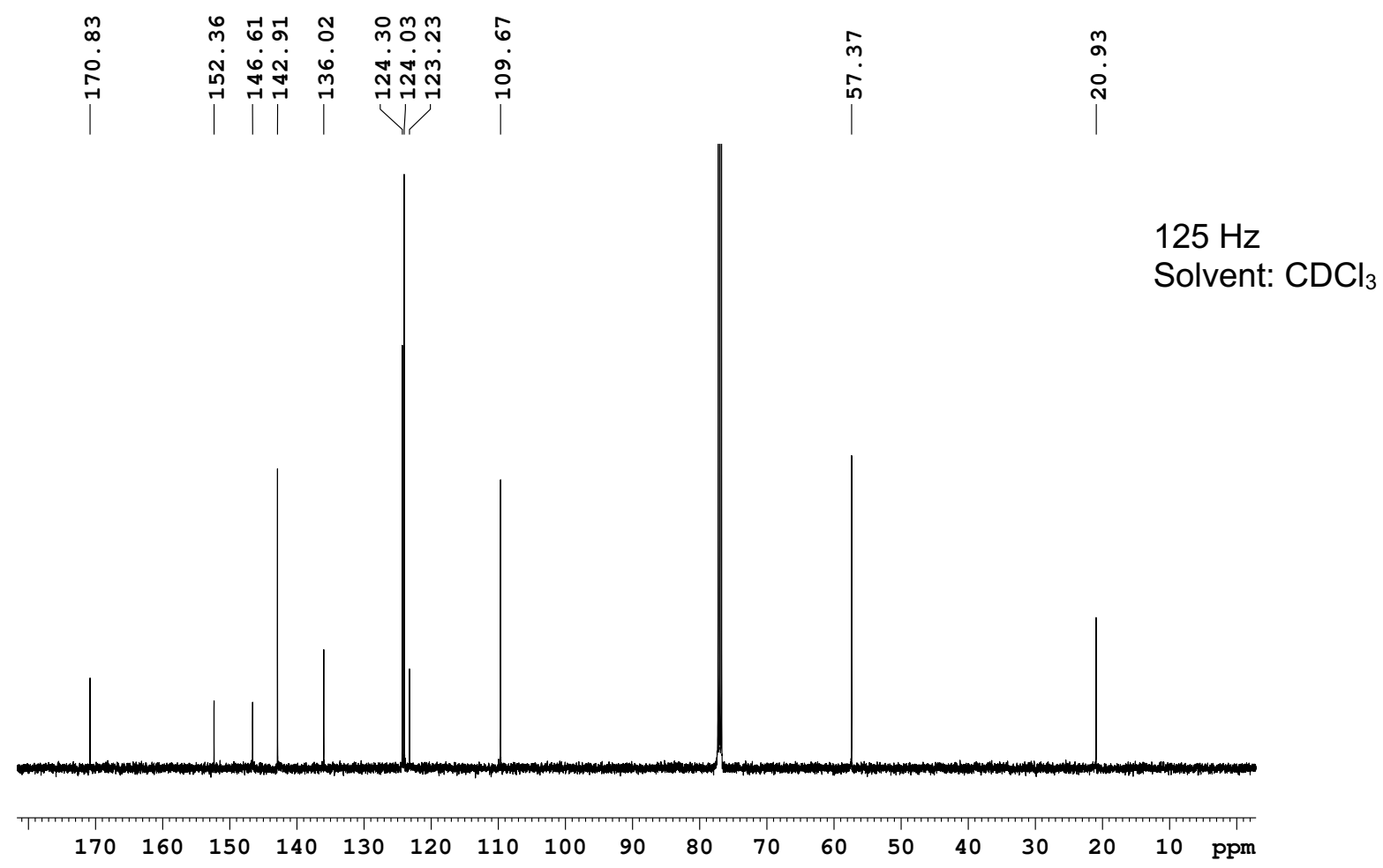




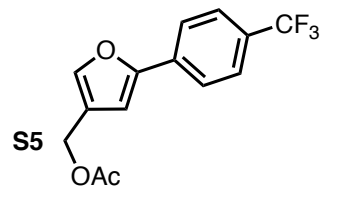

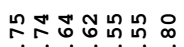

垈桴。

$\stackrel{\overrightarrow{0}}{i n}$
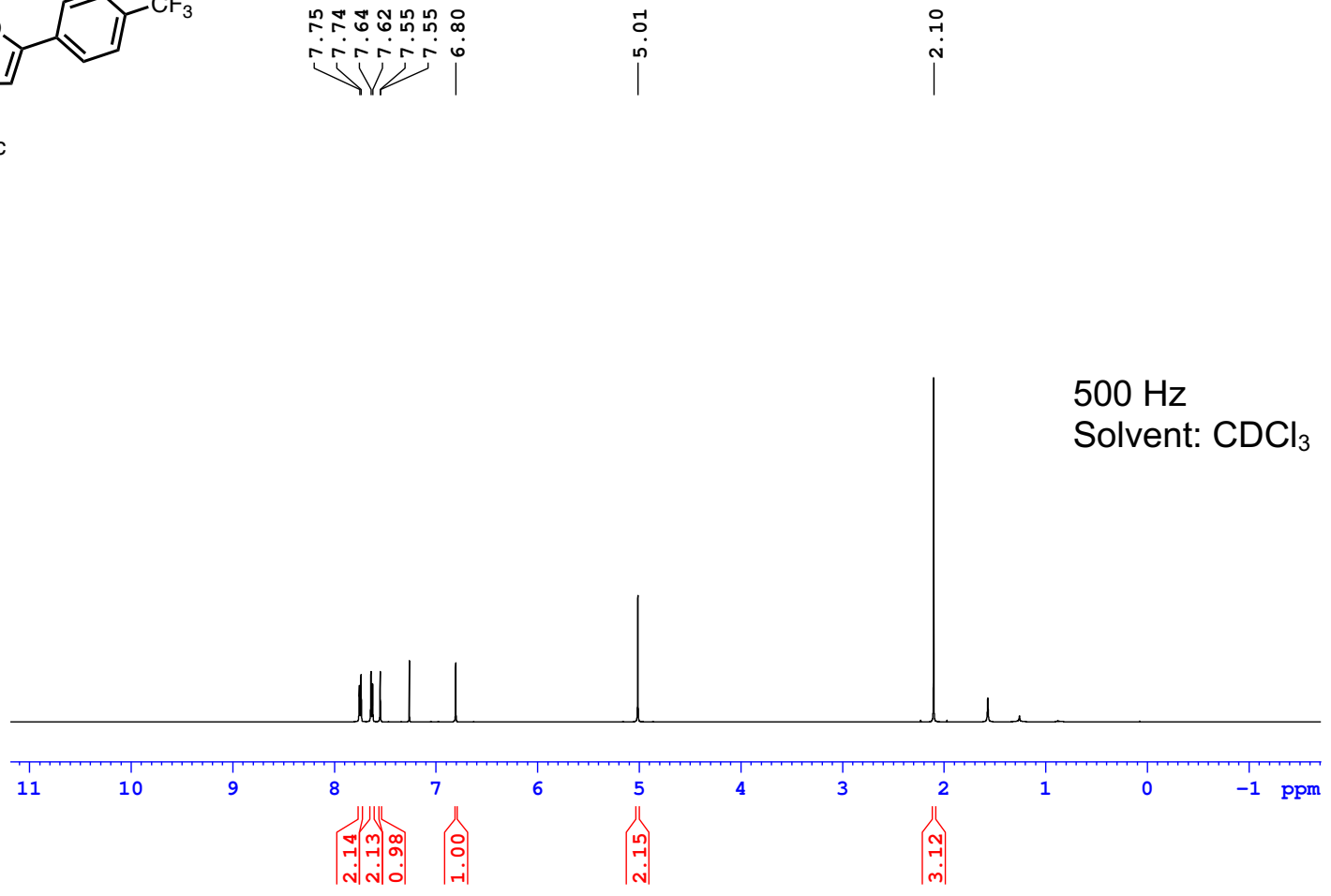

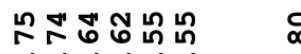

iritis o

to

ก่

우 ‥

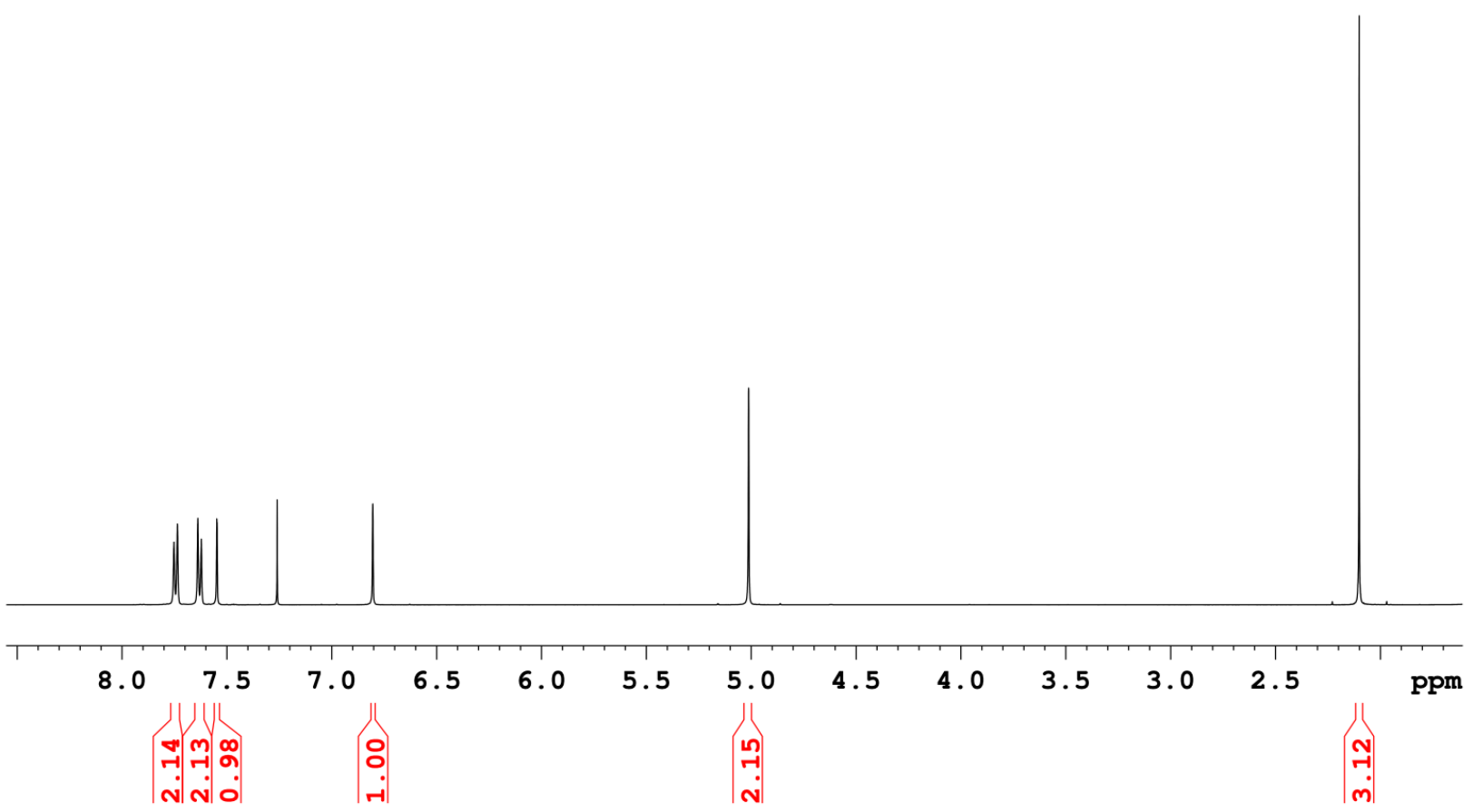


<smiles>CC(=O)OCc1coc(-c2ccc(C(F)(F)F)cc2)c1</smiles>

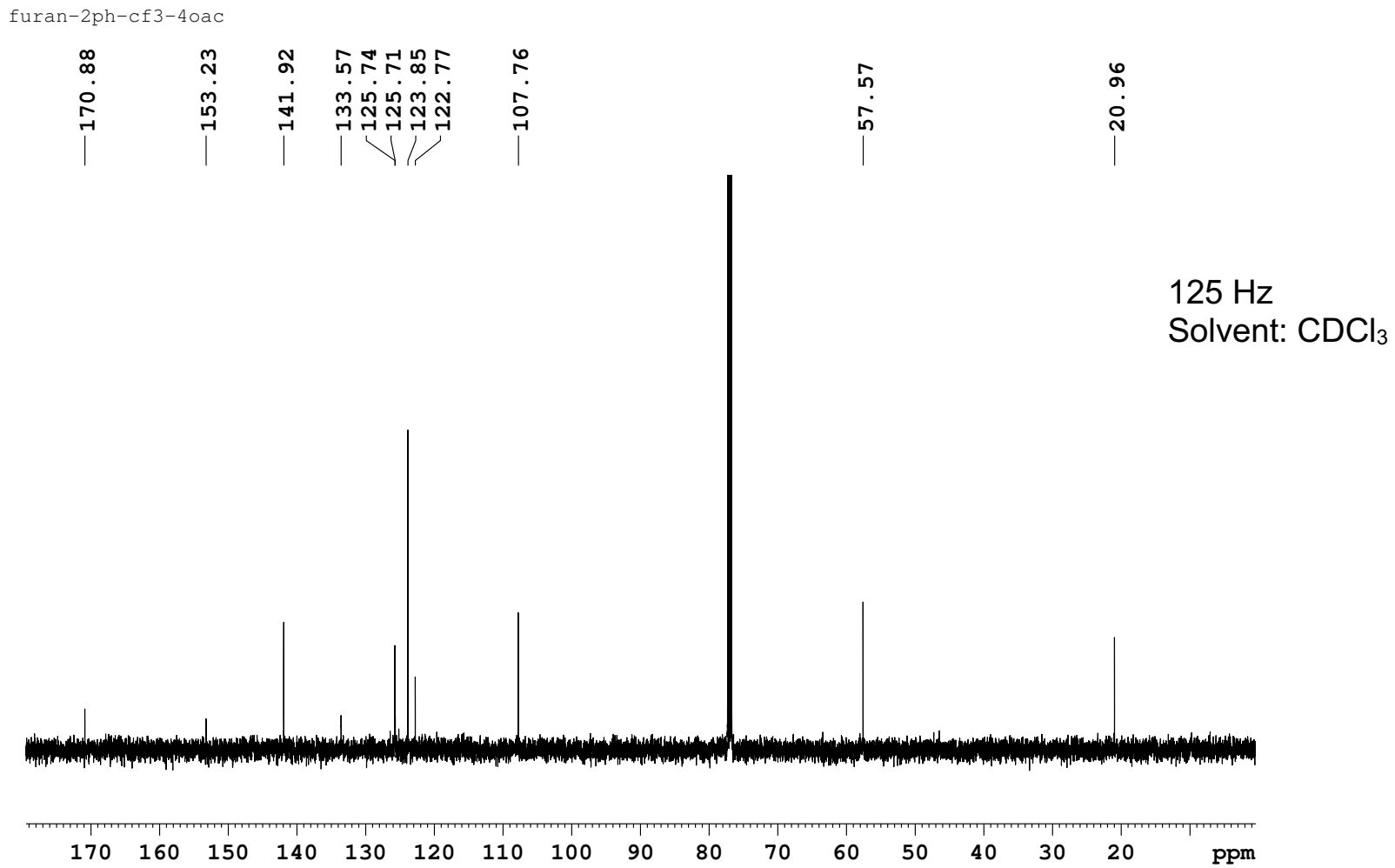


$\sum_{O A C}^{O}$

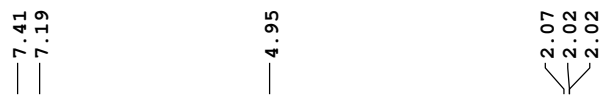

$500 \mathrm{~Hz}$

Solvent: $\mathrm{CDCl}_{3}$

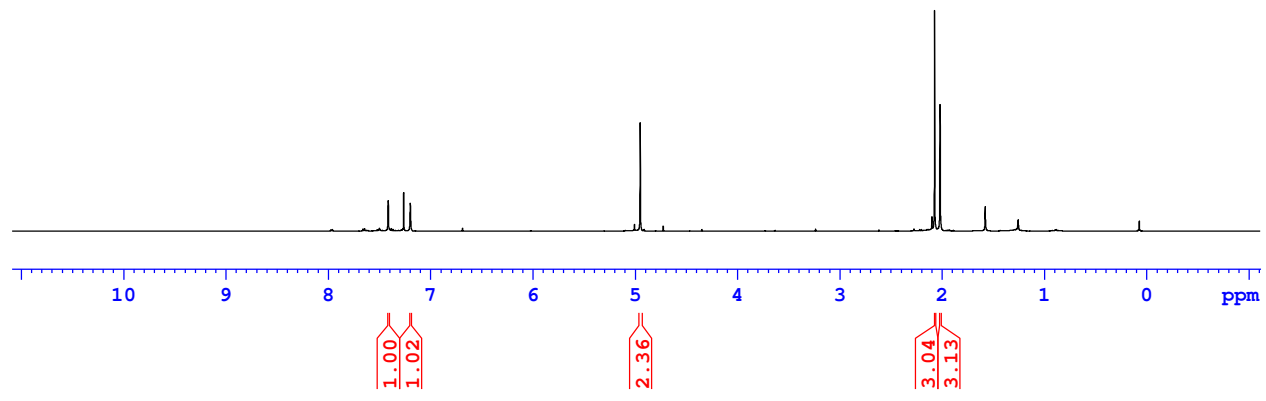

fur-4oac-3me

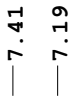

ณ

กับำ

N

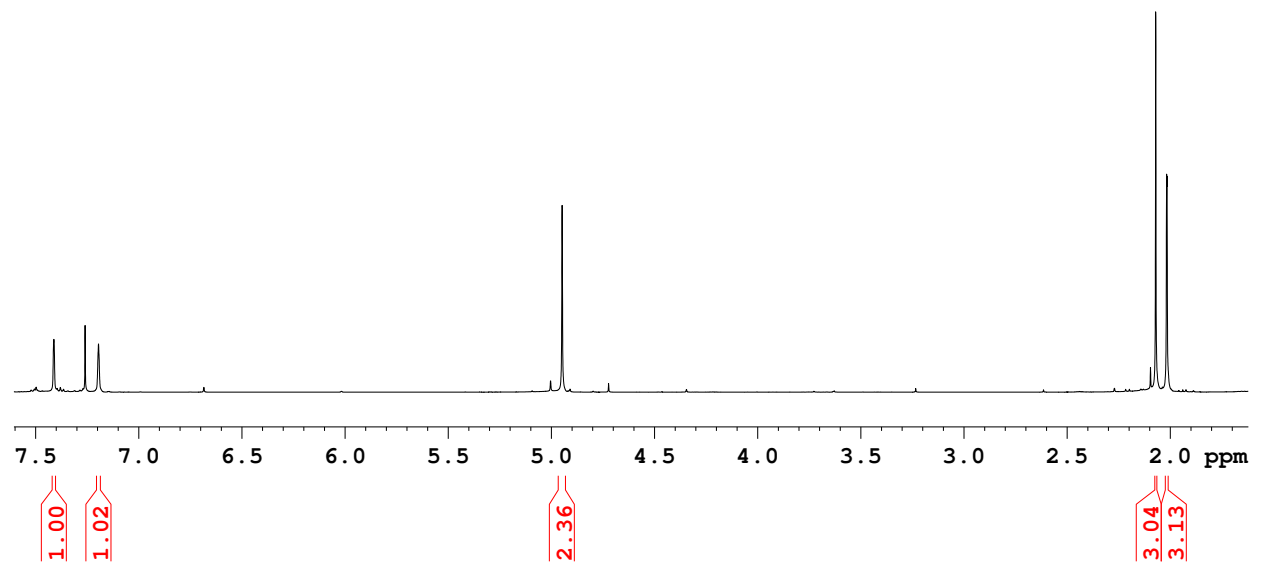


$\int_{\text {OAc }}^{\infty}$

4oac-3me furan

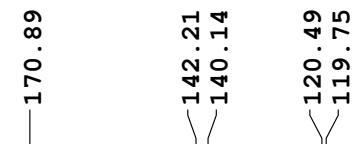

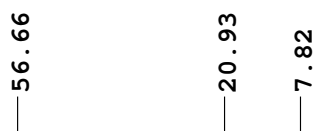

$125 \mathrm{~Hz}$

Solvent: $\mathrm{CDCl}_{3}$

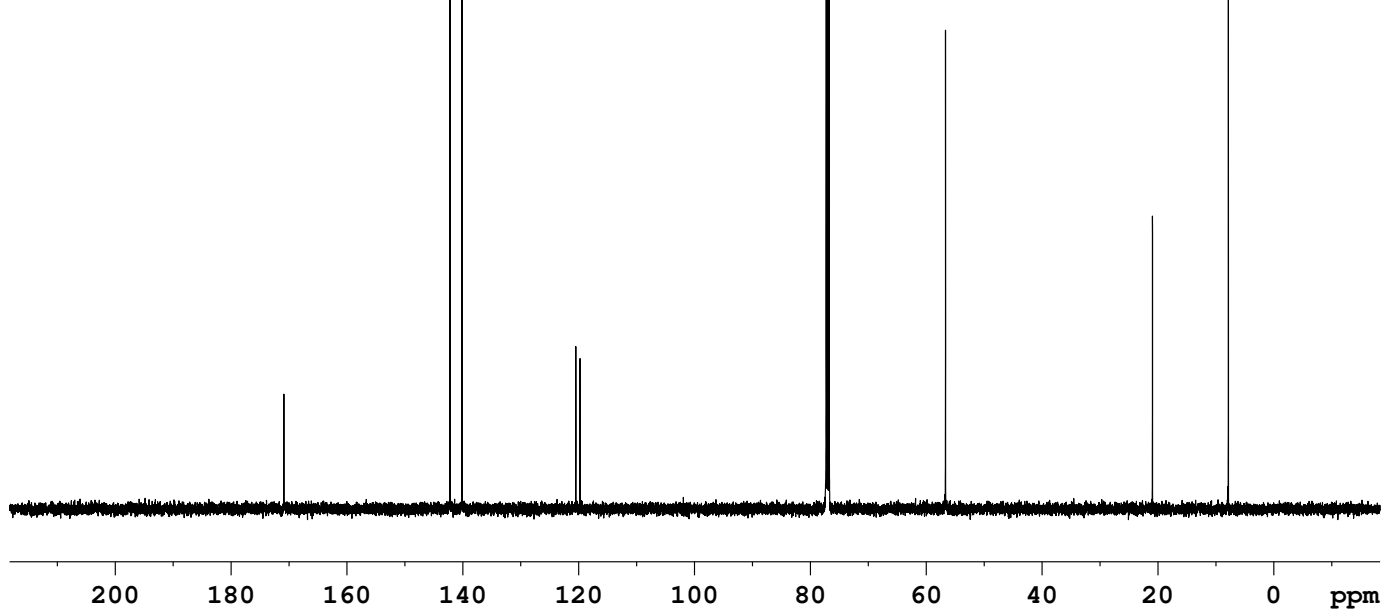


<smiles>CC(=O)OCc1occc1Cc1ccccc1</smiles>

fur-2-oac-3bz

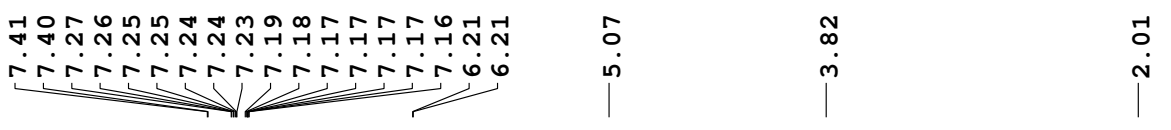

$500 \mathrm{~Hz}$

Solvent: $\mathrm{CD}_{3} \mathrm{OD}$

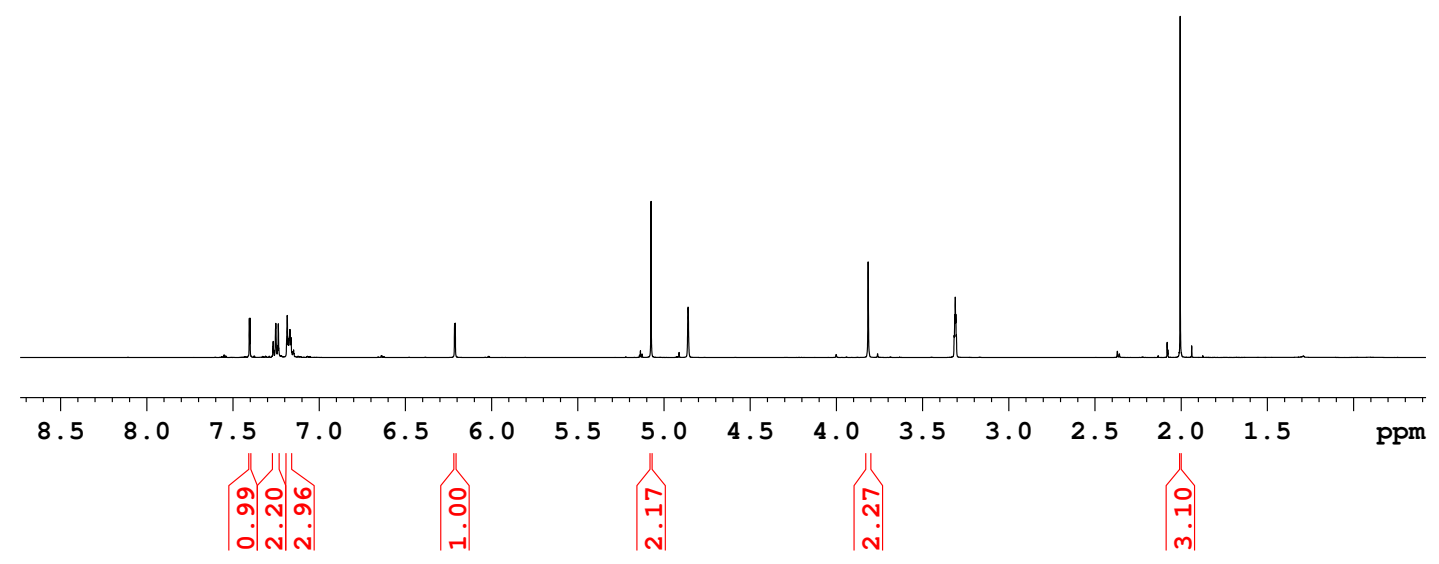


<smiles>CC(=O)OCc1occc1Cc1ccccc1</smiles>

fur $-2-0 a c-3 b z$

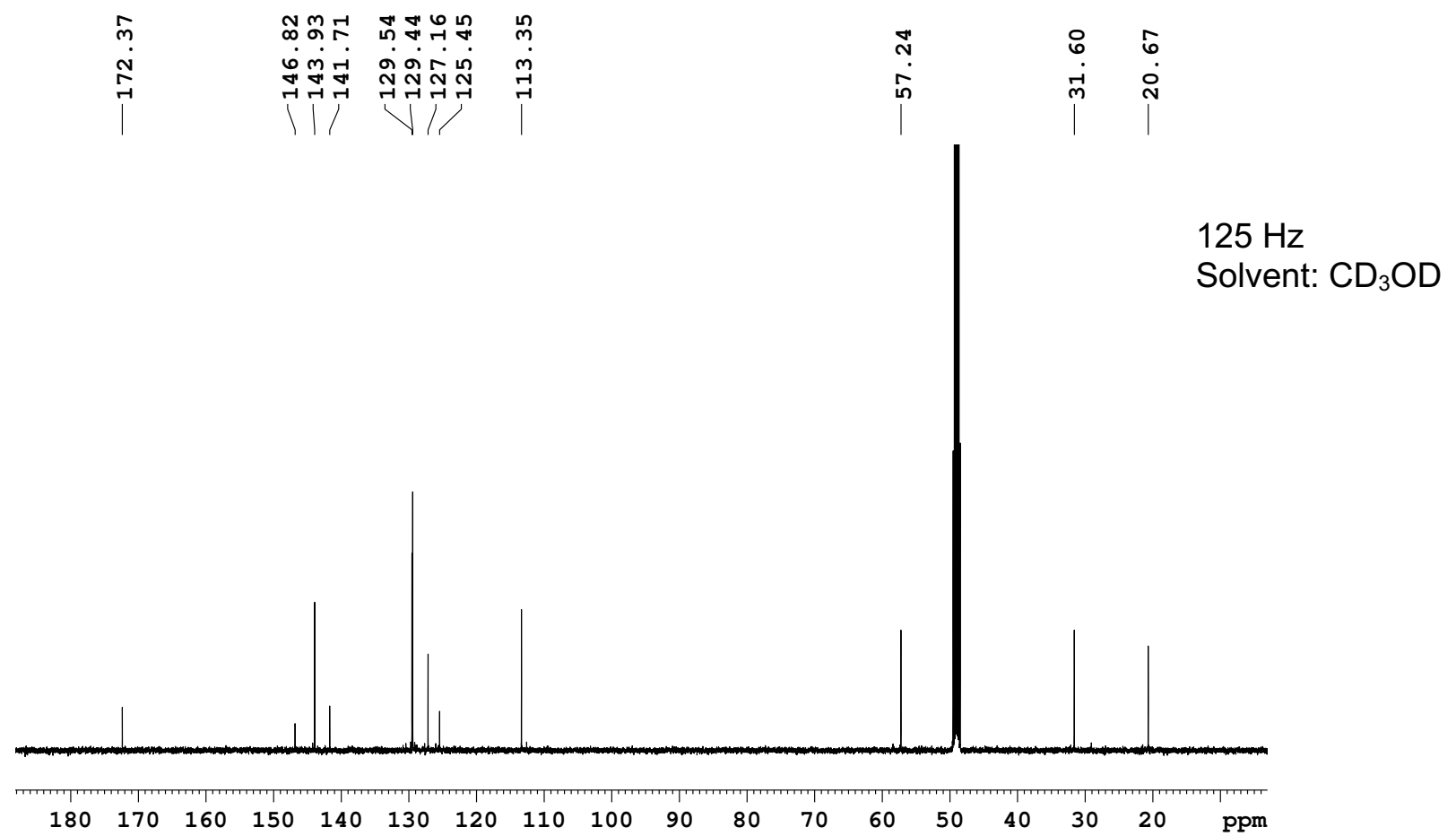



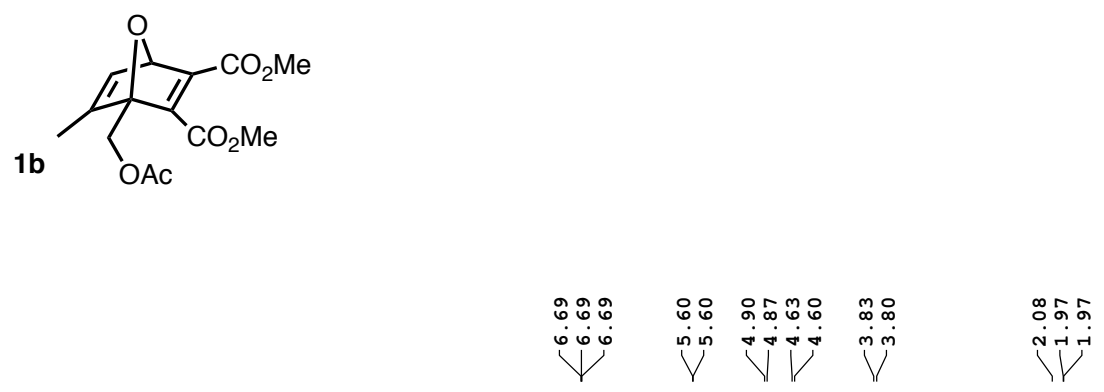

$500 \mathrm{~Hz}$

Solvent: $\mathrm{CDCl}_{3}$

ond-2-oac-3me

\begin{tabular}{|c|c|c|c|c|}
\hline ํํㅇํํ & $: 0$ & 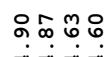 & 冓 & ๓๐็์ \\
\hline$\dot{V}$ & $v^{\text {ம் }}$ & Vij & $\eta^{m}$ & Nij \\
\hline
\end{tabular}

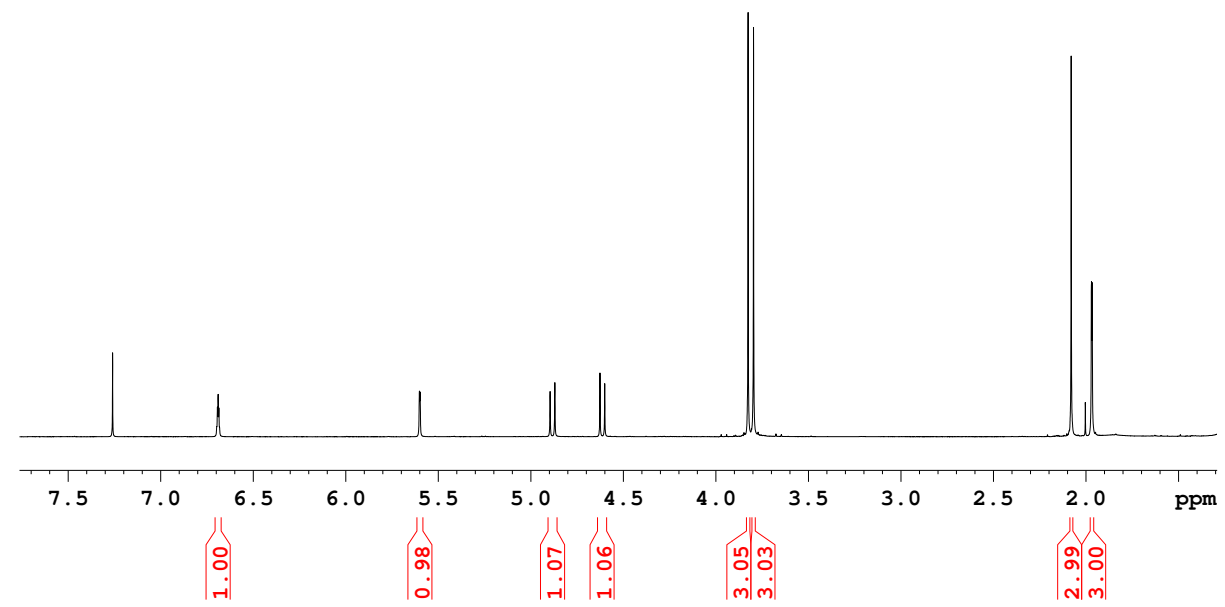




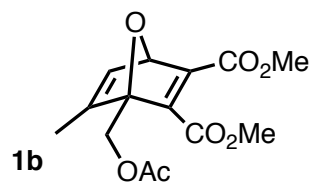

ond-2-oac-3me

ร ํำ ㄱำ

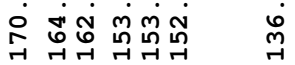

|

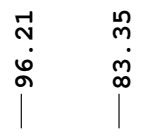

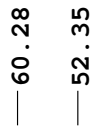

เี่ $\stackrel{\infty}{\circ}$

ก

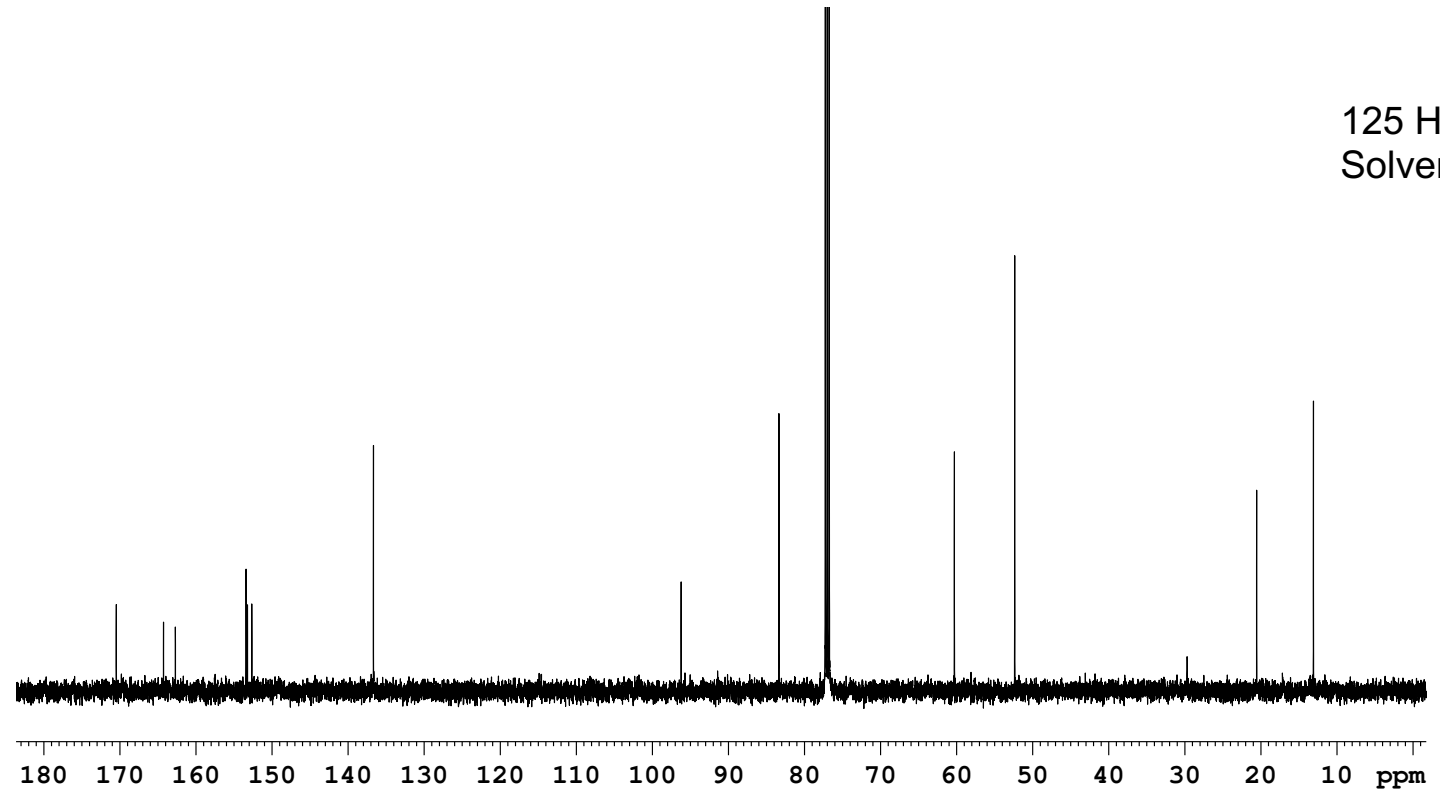



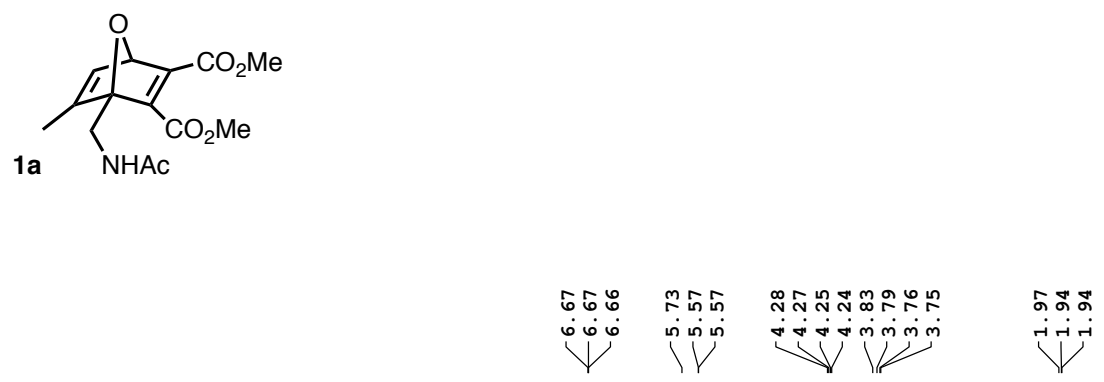

$500 \mathrm{~Hz}$

Solvent: $\mathrm{CDCl}_{3}$
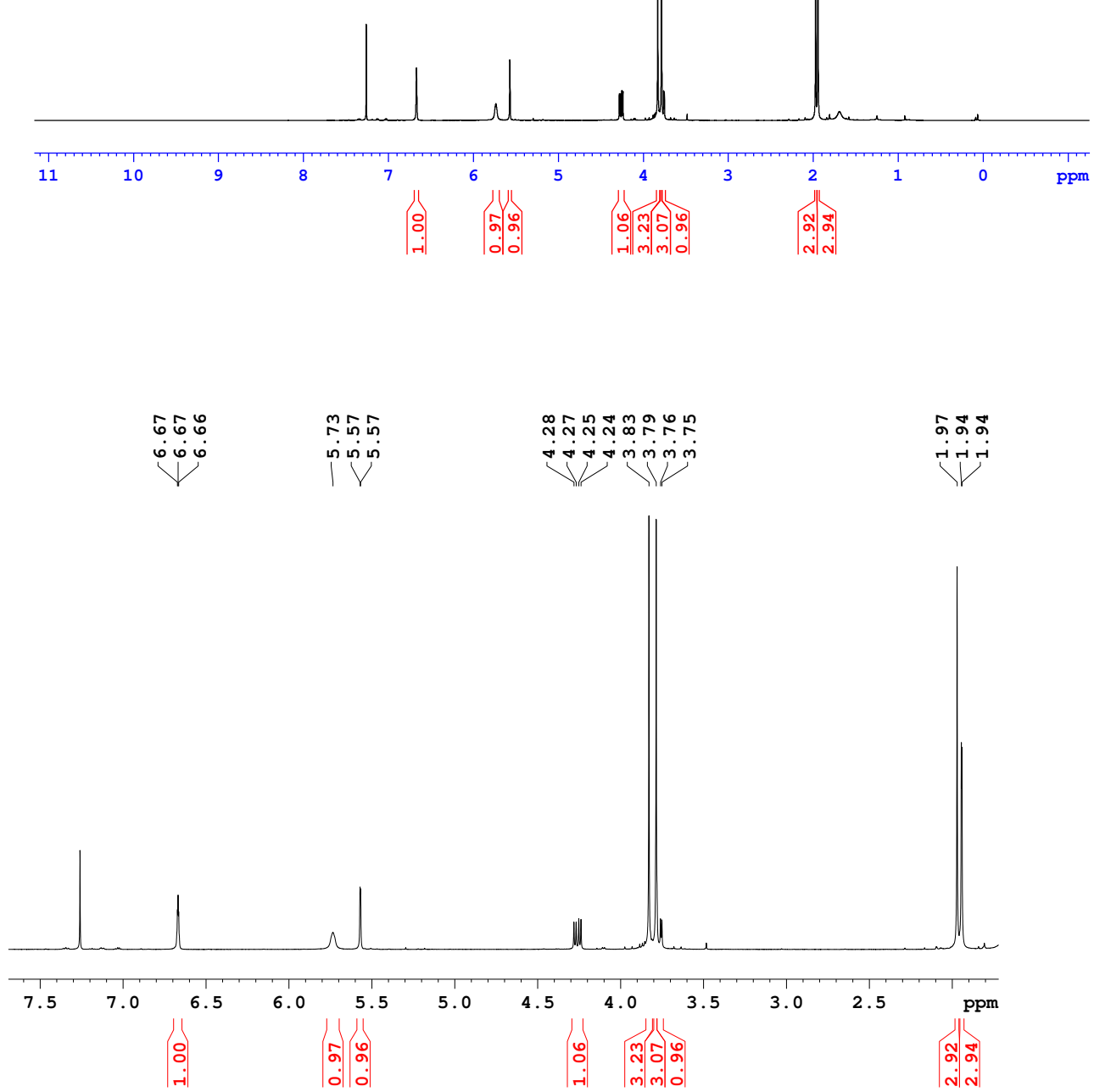

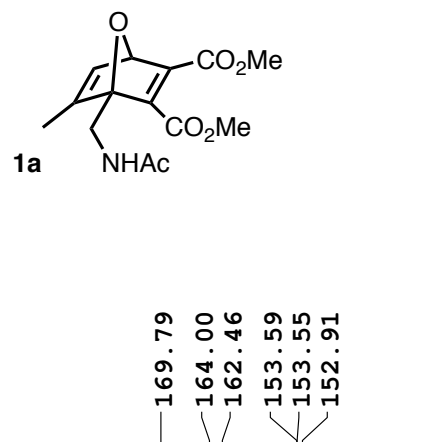

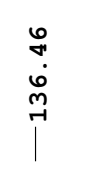

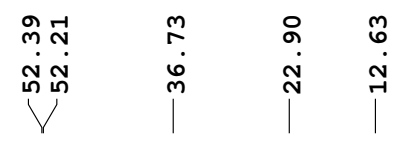

$125 \mathrm{~Hz}$

Solvent: $\mathrm{CDCl}_{3}$
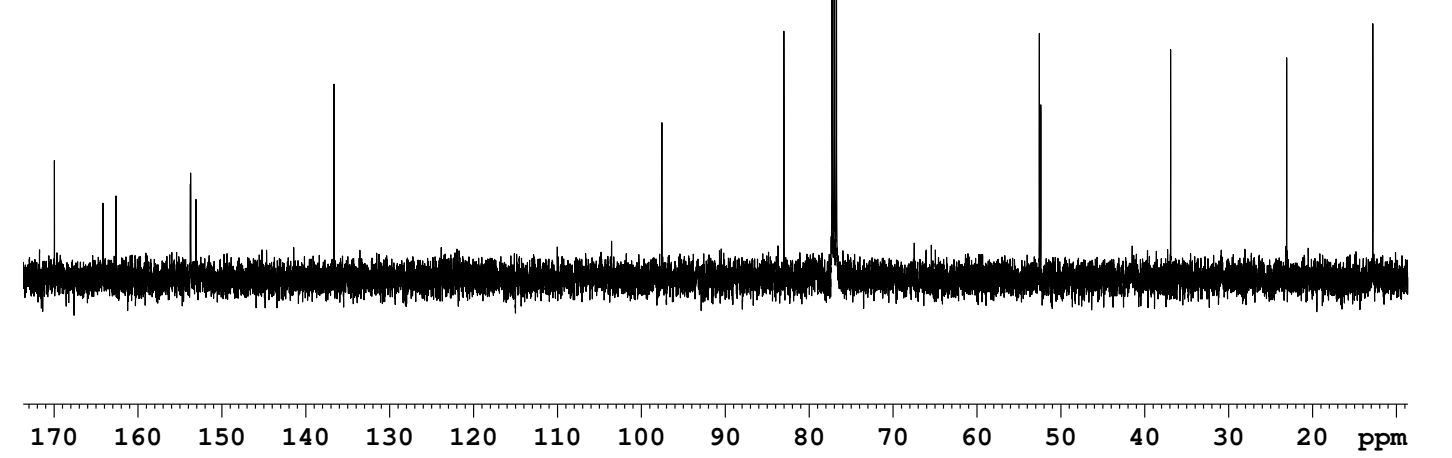

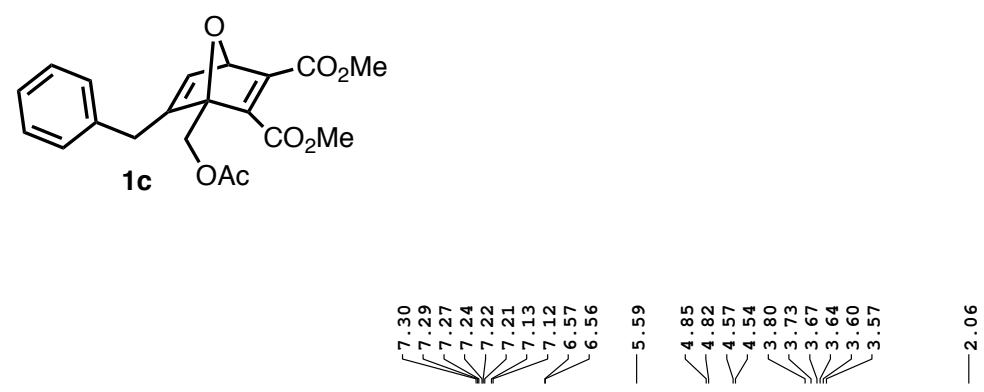

$500 \mathrm{~Hz}$

Solvent: $\mathrm{CDCl}_{3}$

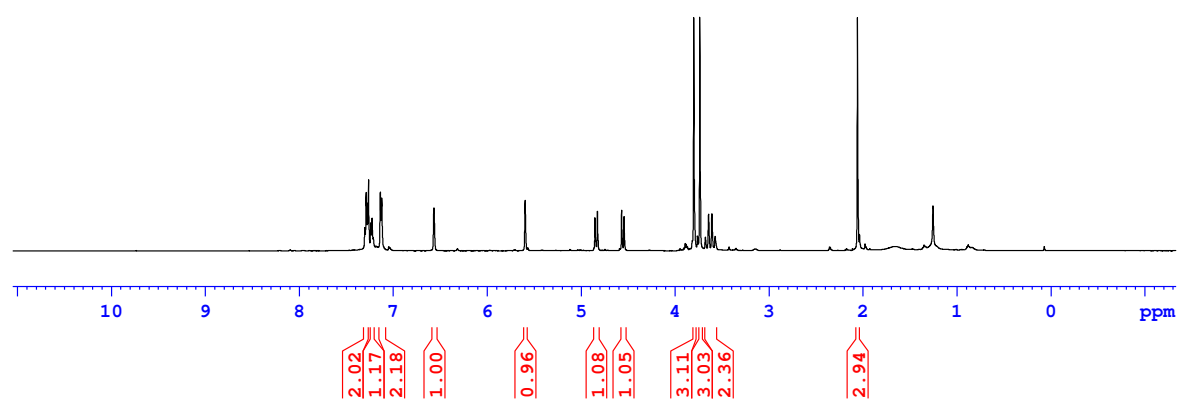

ond $-2-o a c-3-b z$

\begin{tabular}{|c|c|c|c|}
\hline & ำ & ம & 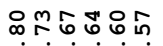 \\
\hline 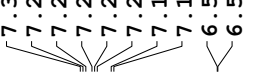 & n! & نன் & $\dot{m} \dot{m} \dot{m} \dot{m}$ \\
\hline
\end{tabular}

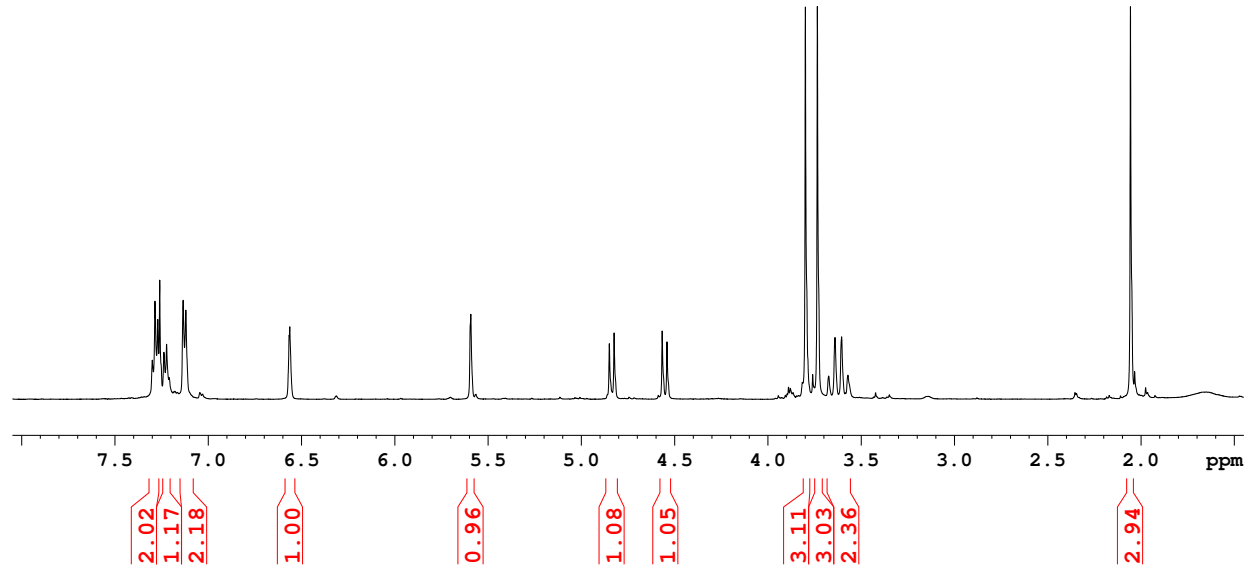




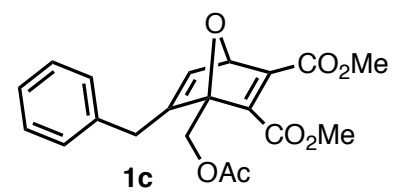

ond $-2-o a c-3-b z$

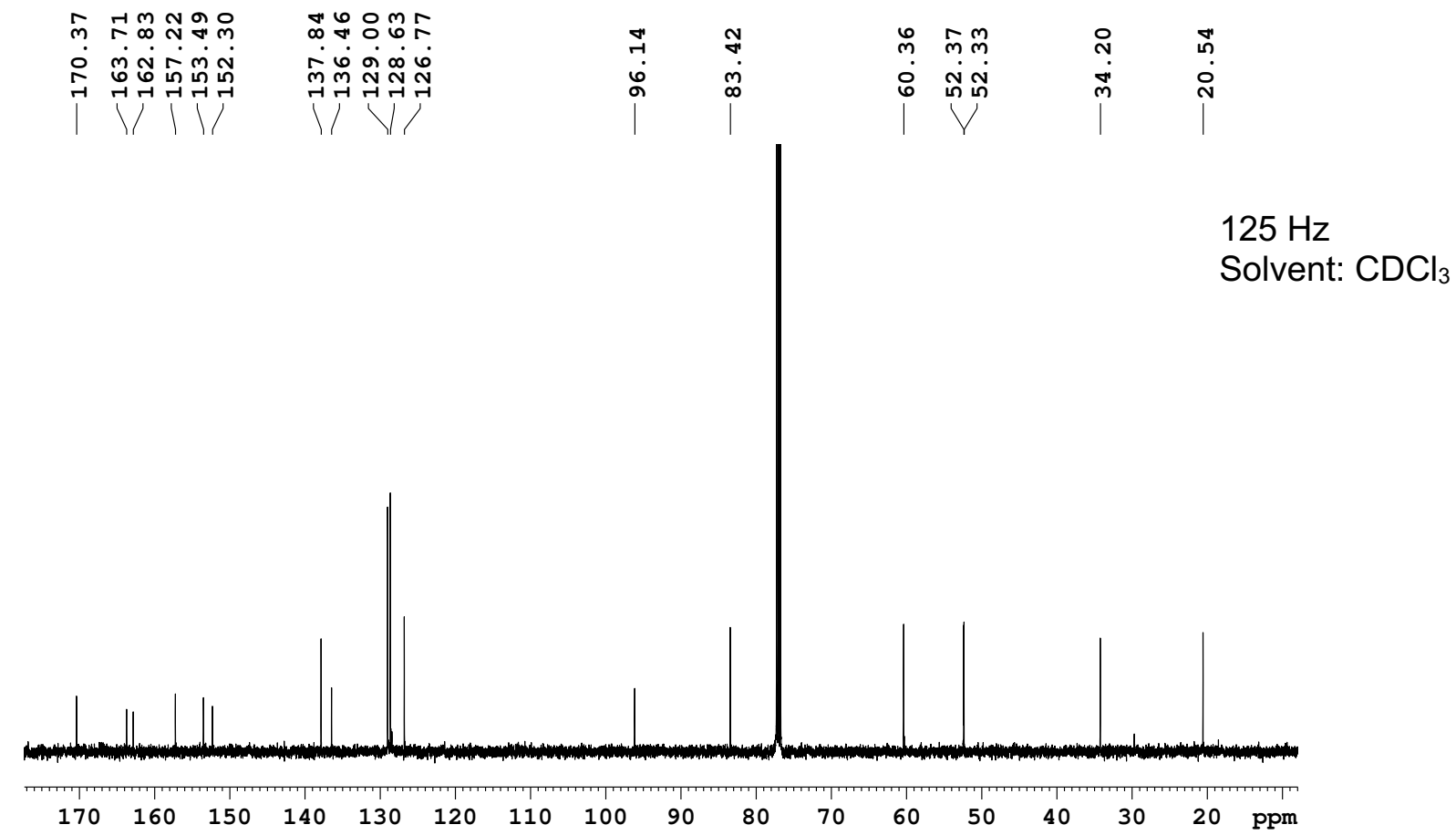




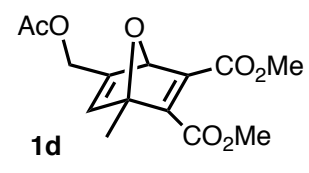

4 oac-2me ond

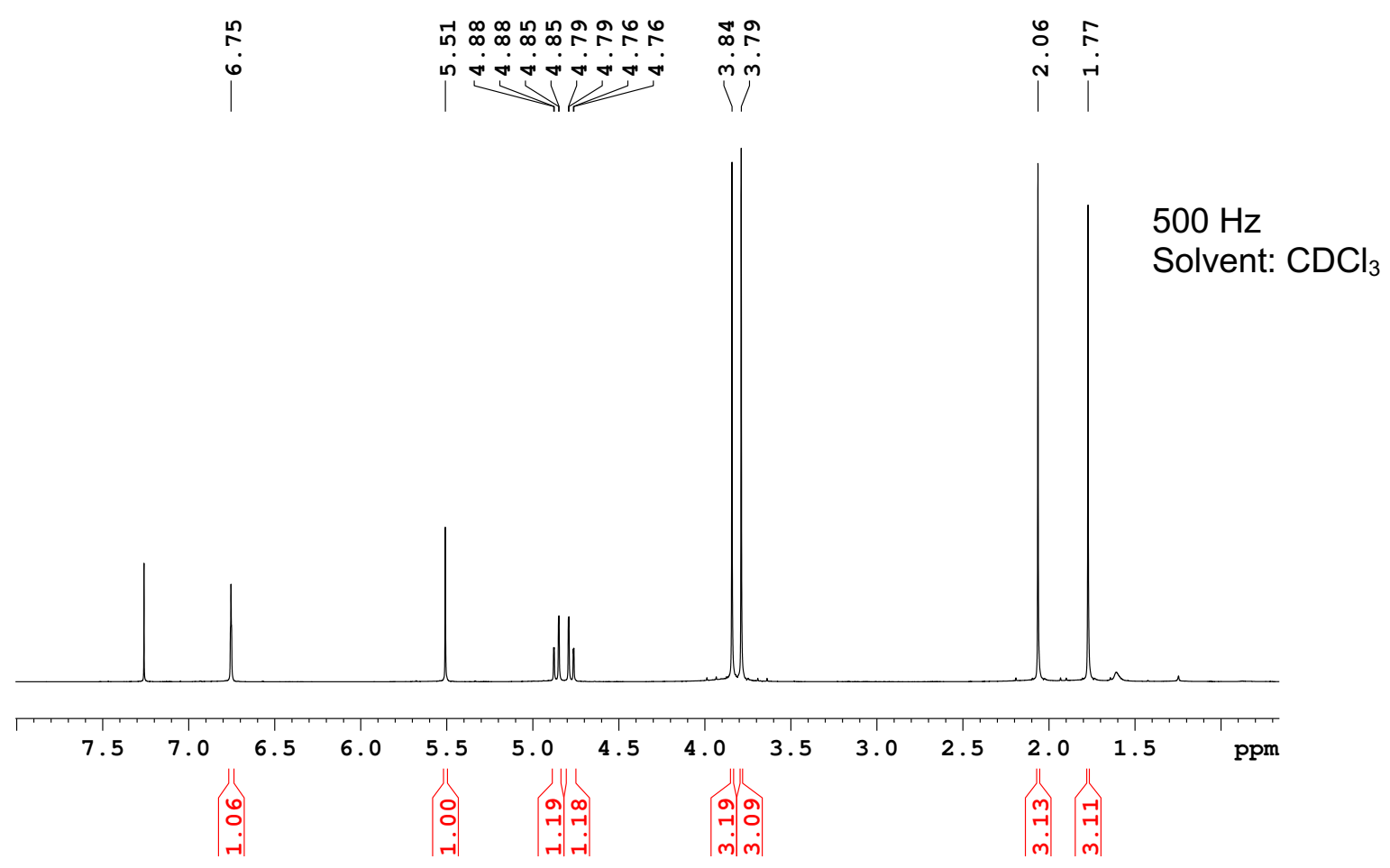




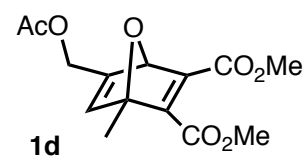

ond-2-me-4oac

ณูํํํำก ผ

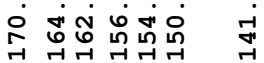

|

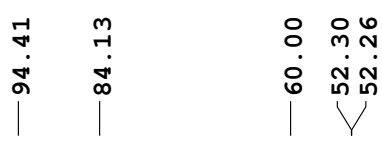

ㄱ. ำ

กั นา

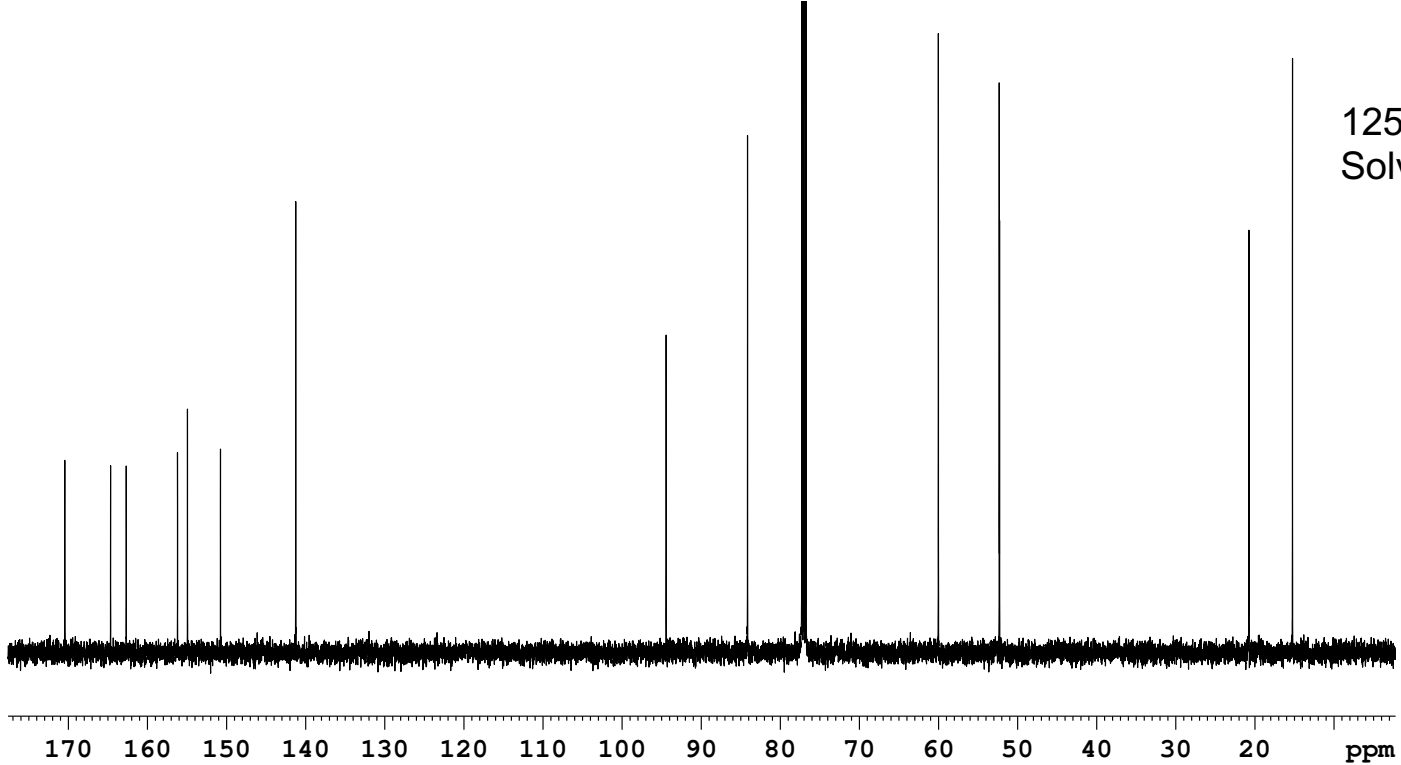




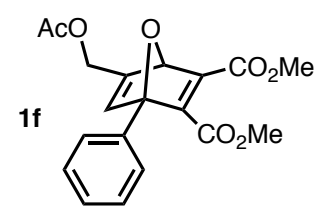

ond $-4 \circ \mathrm{ac}-2 \mathrm{ph}$

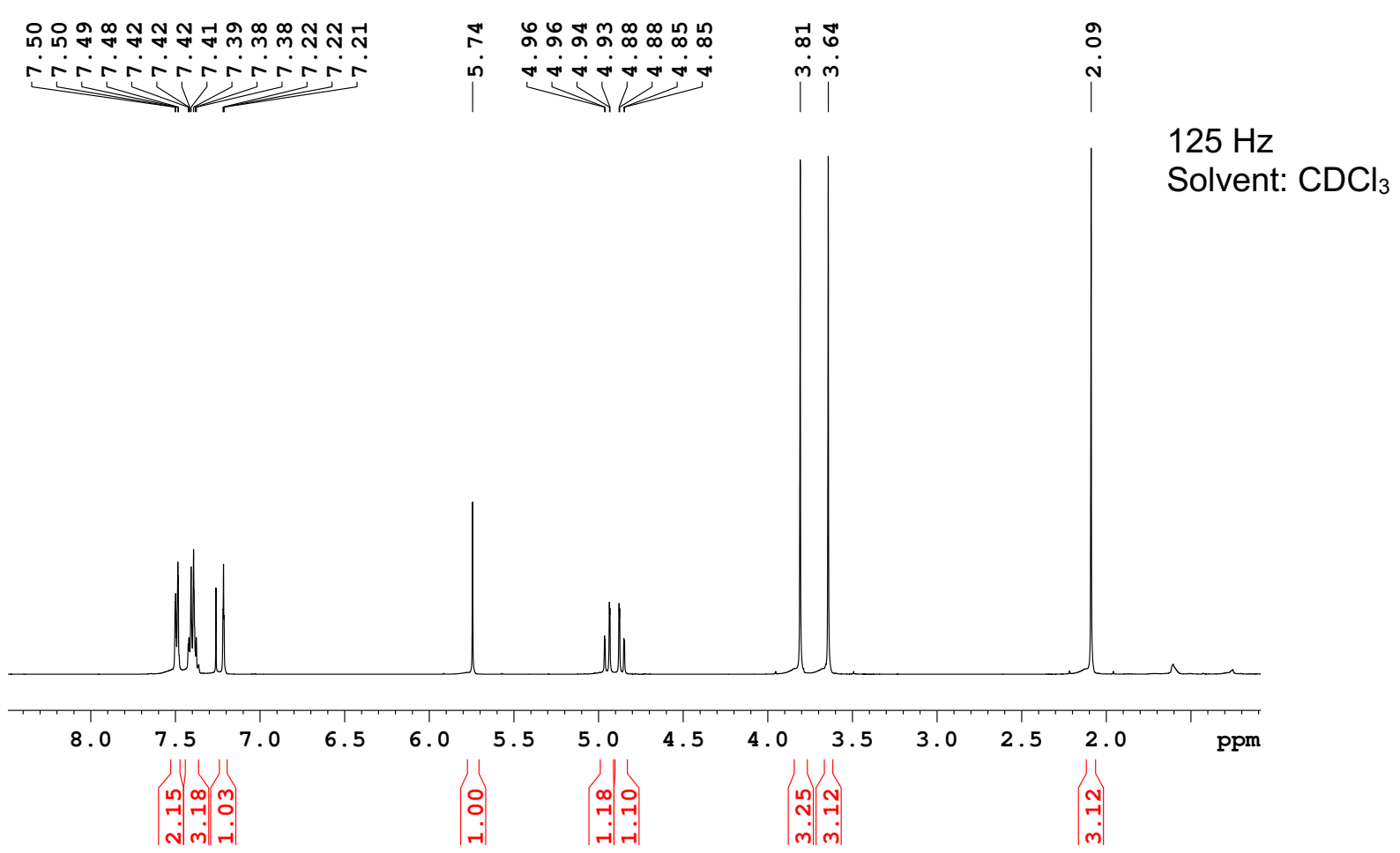




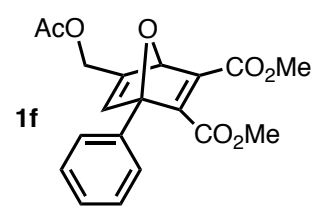

4-oac-2-ph ond

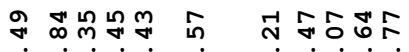

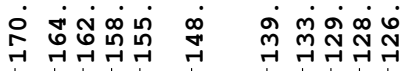

1। ।

مْ

ๆ พูก

จ กิ่ง

$\stackrel{-1}{\infty}$

กั

|

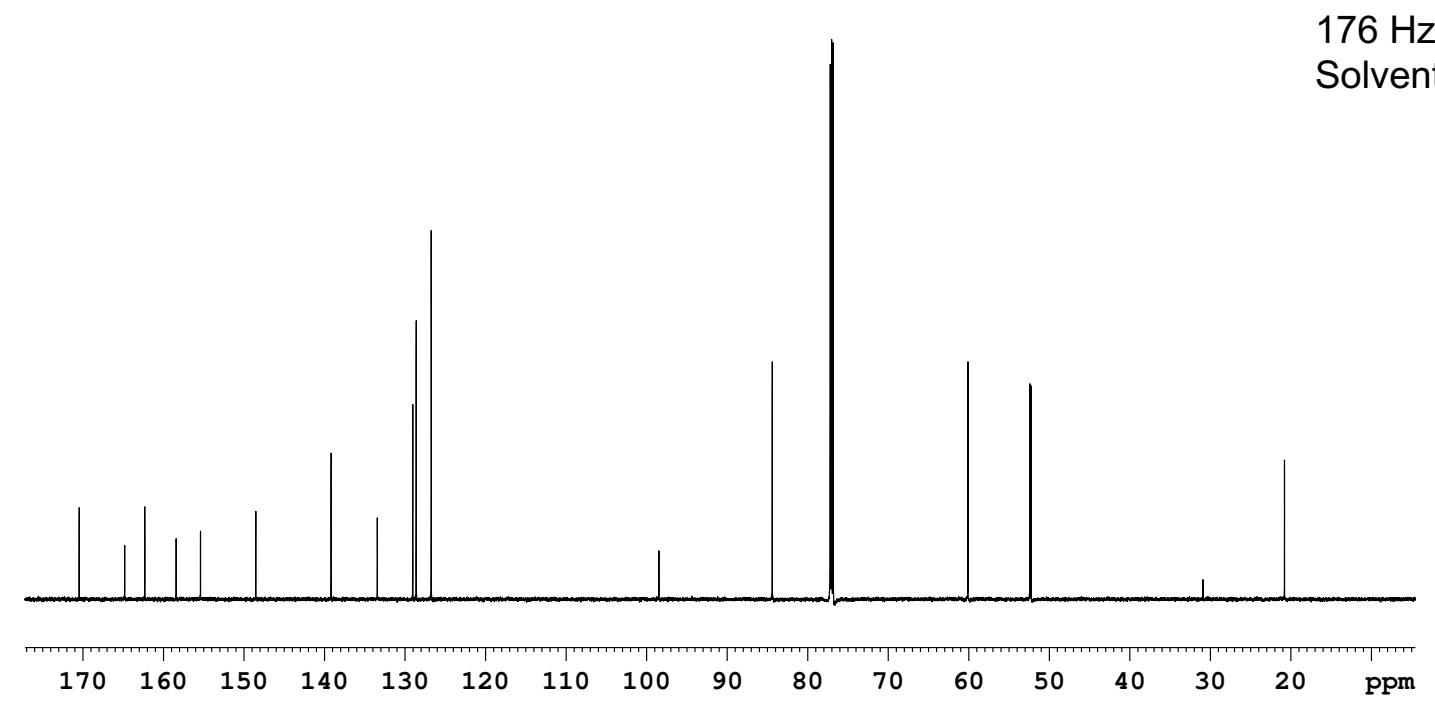




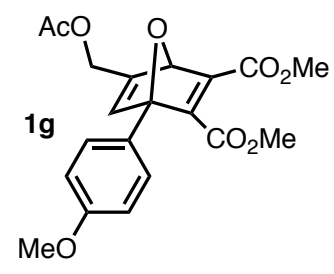

ond-2ph-ome-4oac

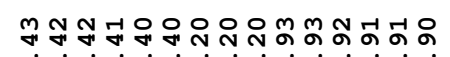
arinaringo $\longrightarrow$

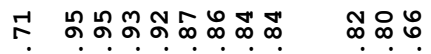

ம $\dot{\nabla} \nabla \dot{\nabla} \nabla \dot{\nabla} \dot{\nabla m}$

VI
오.

N

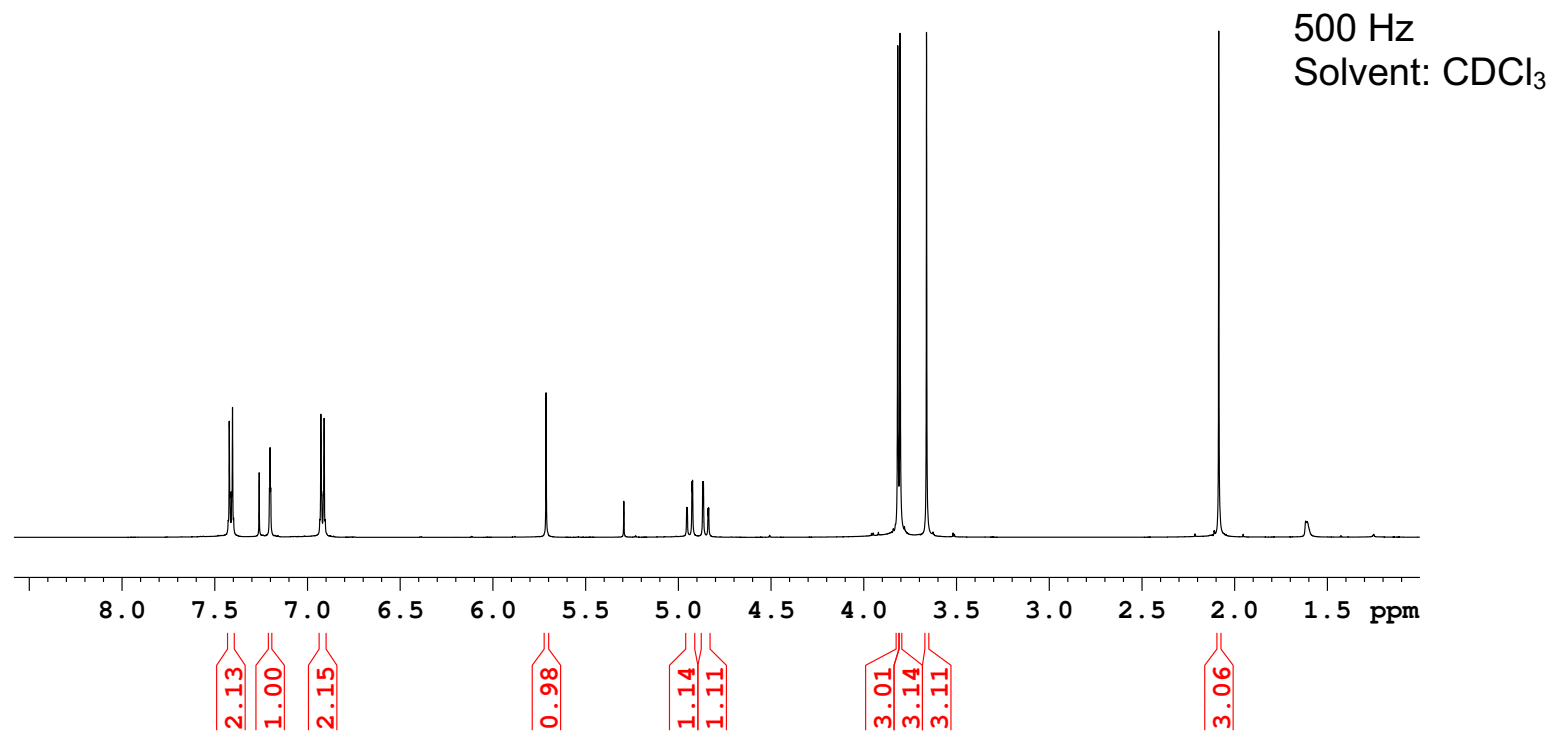



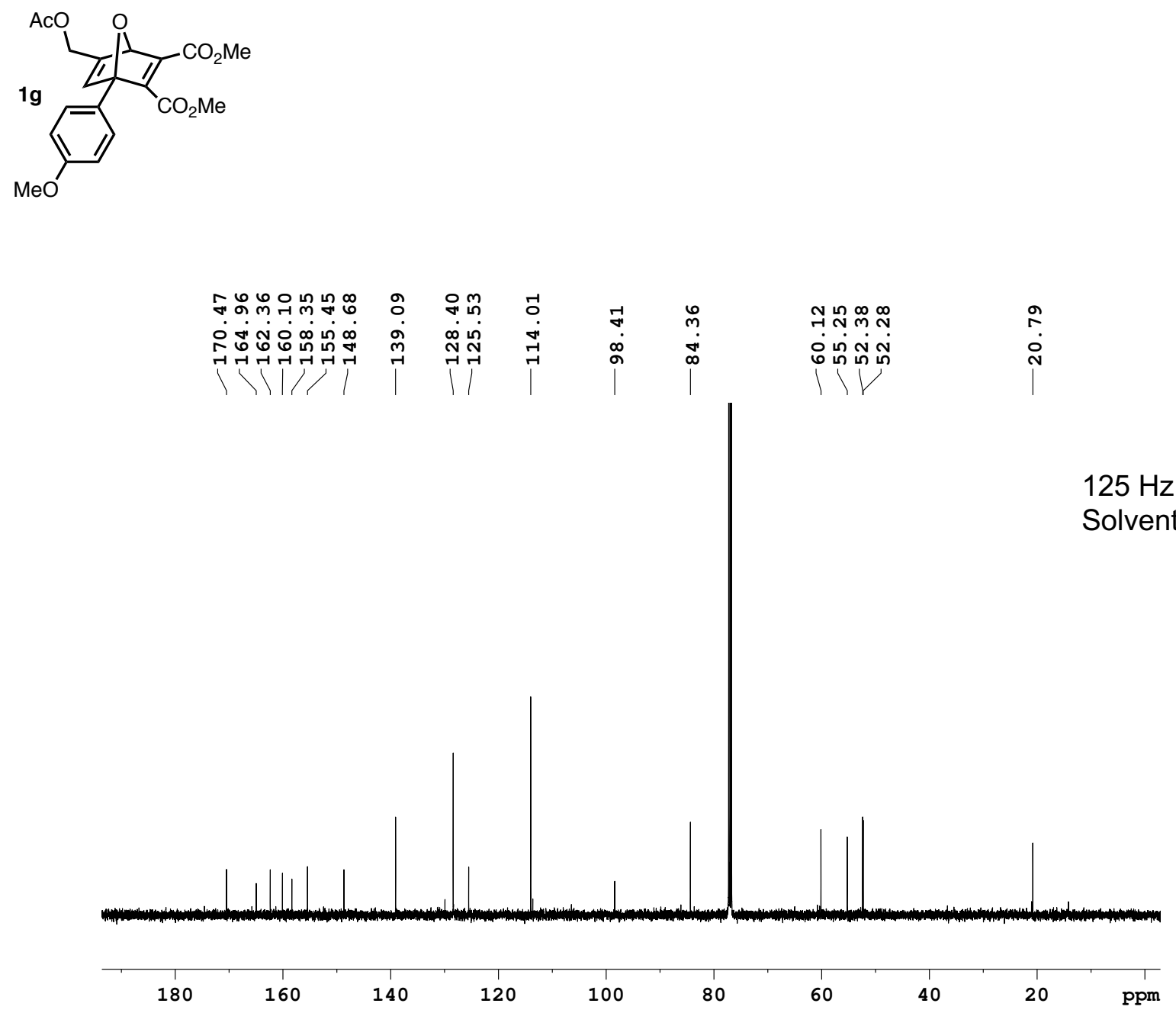

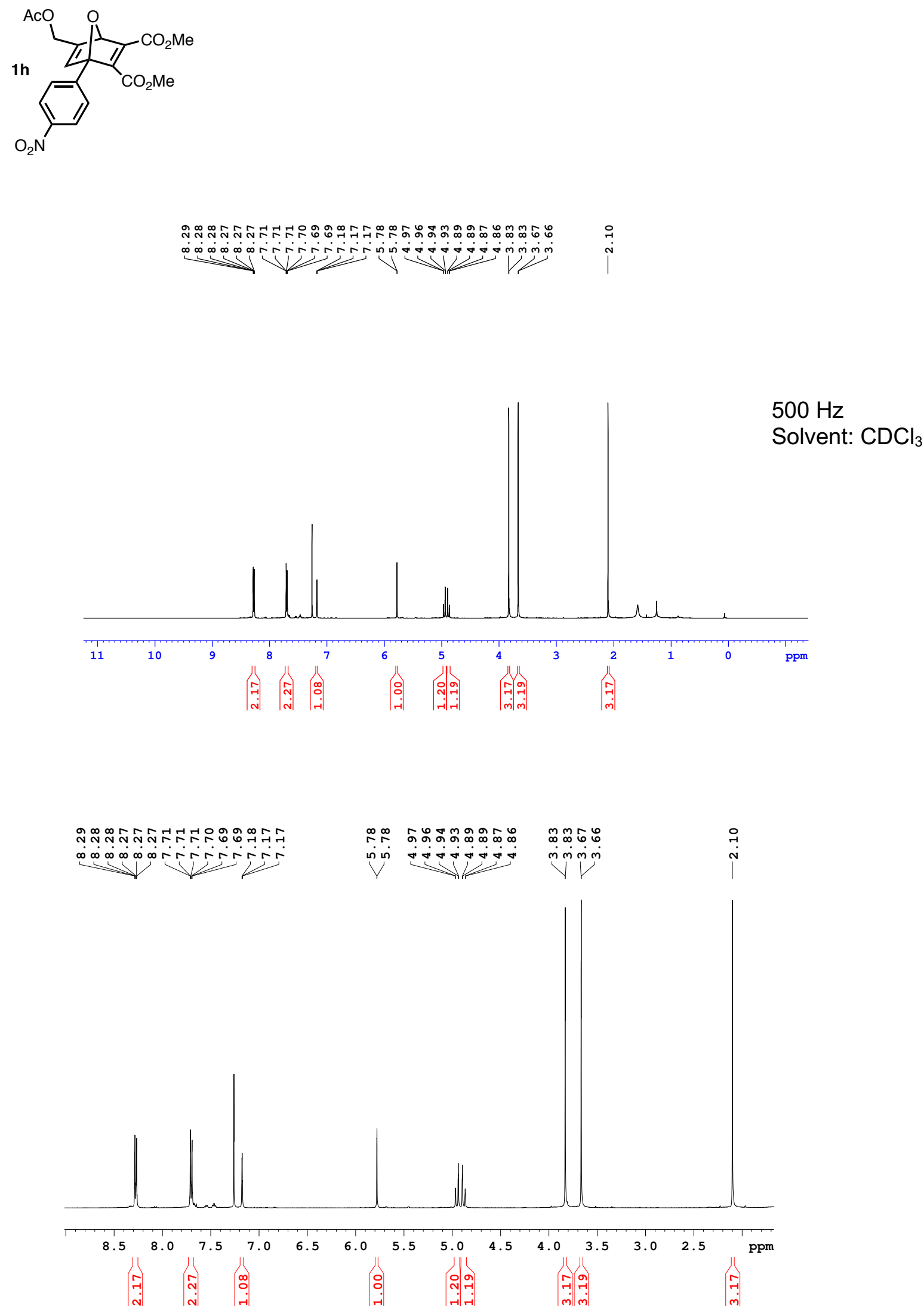


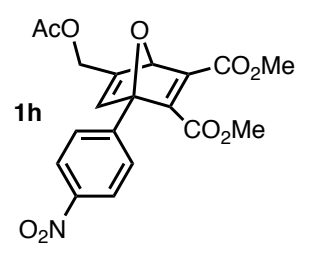

4 oac-2phno2 ons
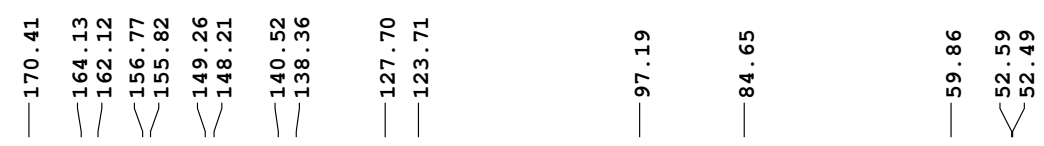

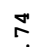

ก

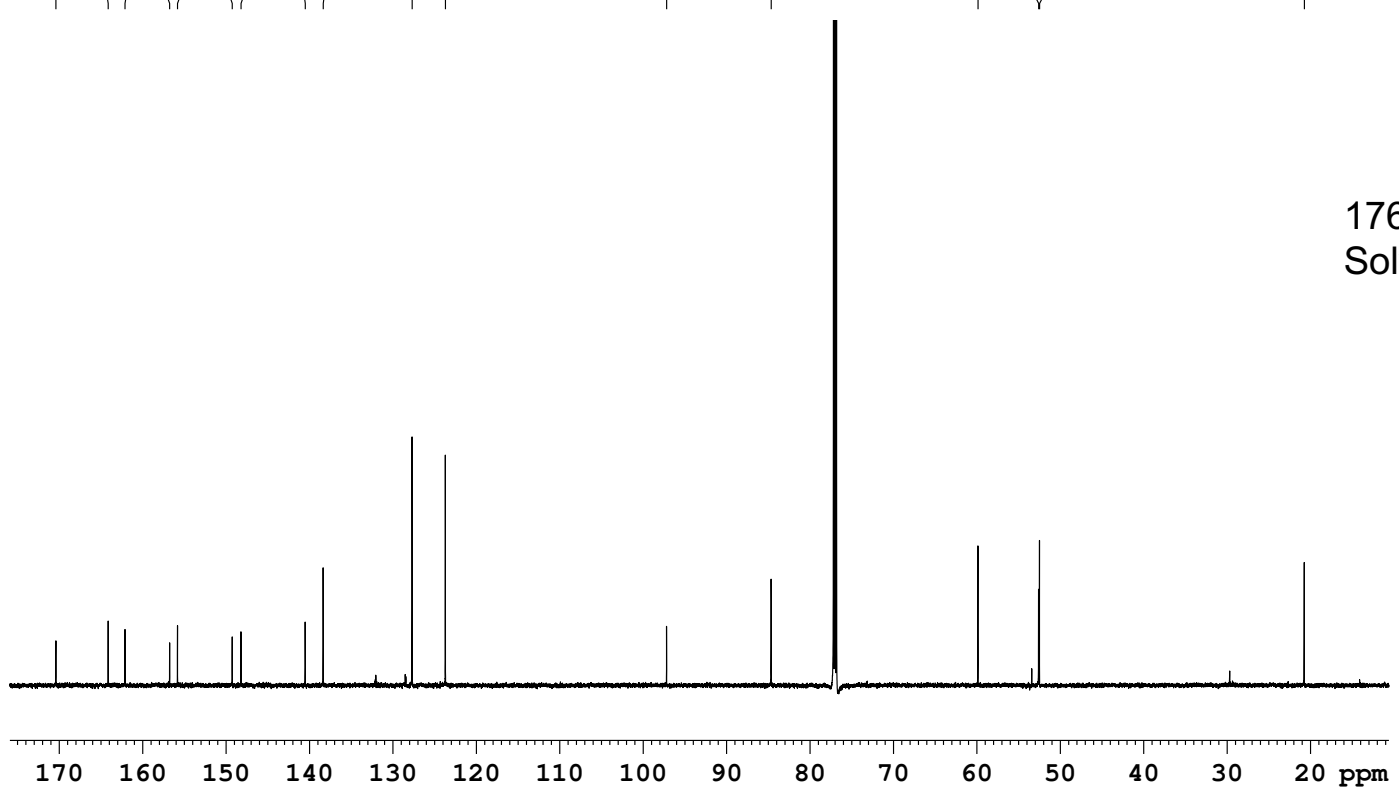




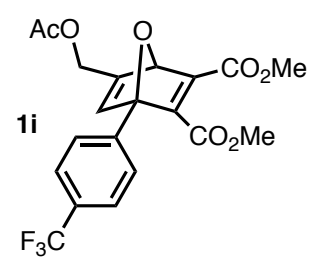

ond-2-ph-cf3-4-oh

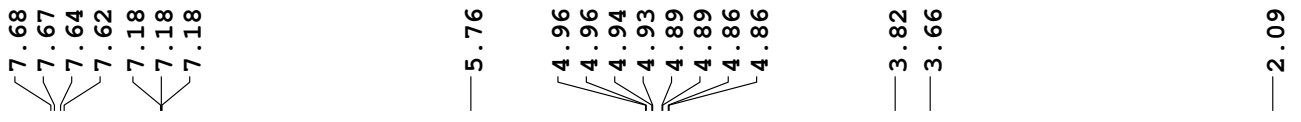

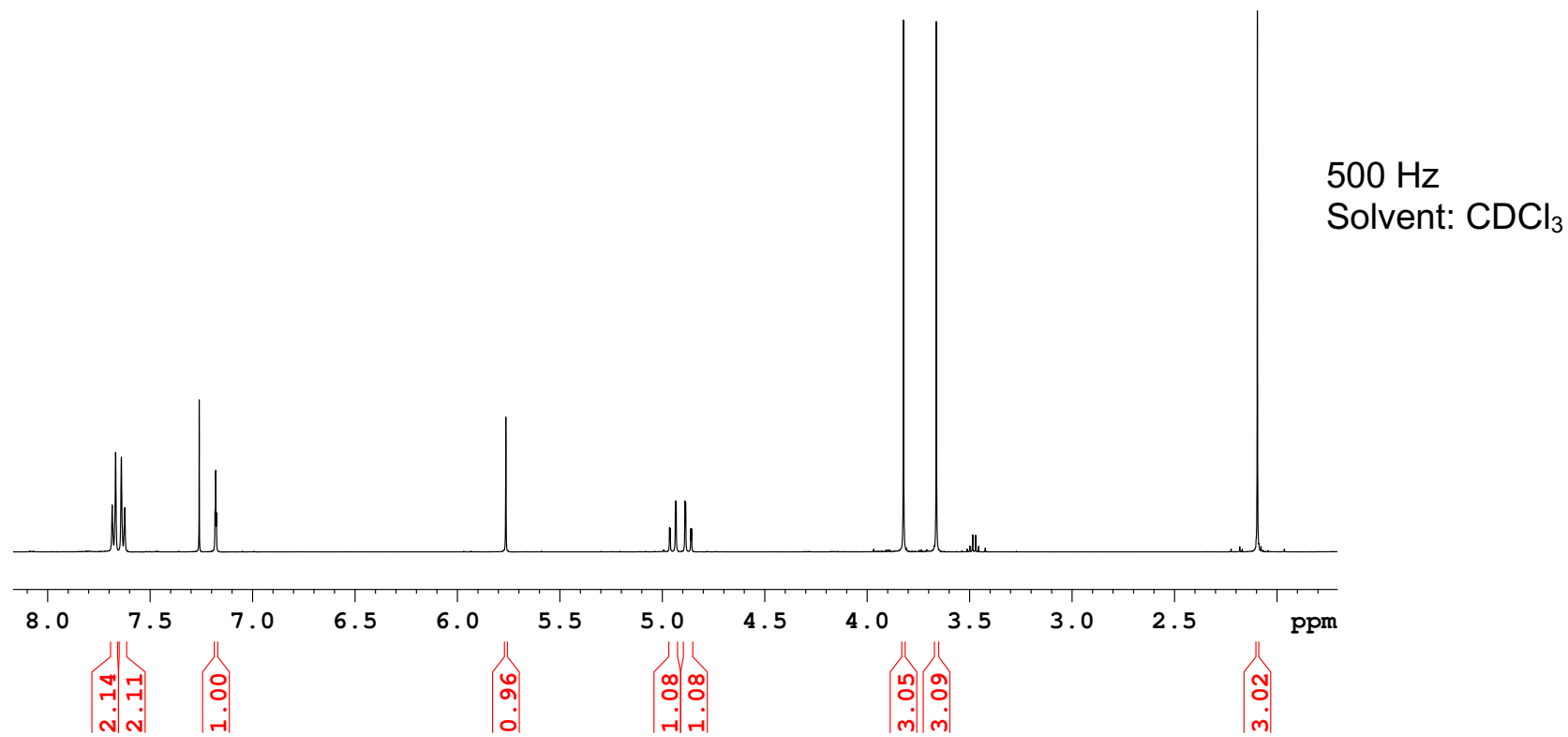




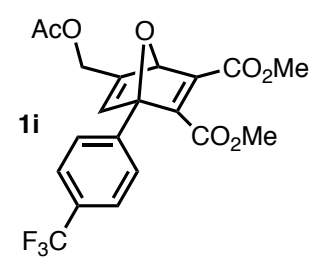

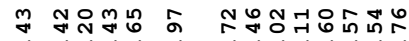

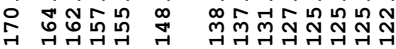

11111111

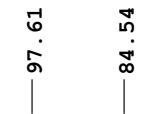

ถำรี

ถิ่ กิ่

|

$125 \mathrm{~Hz}$

Solvent: $\mathrm{CDCl}_{3}$

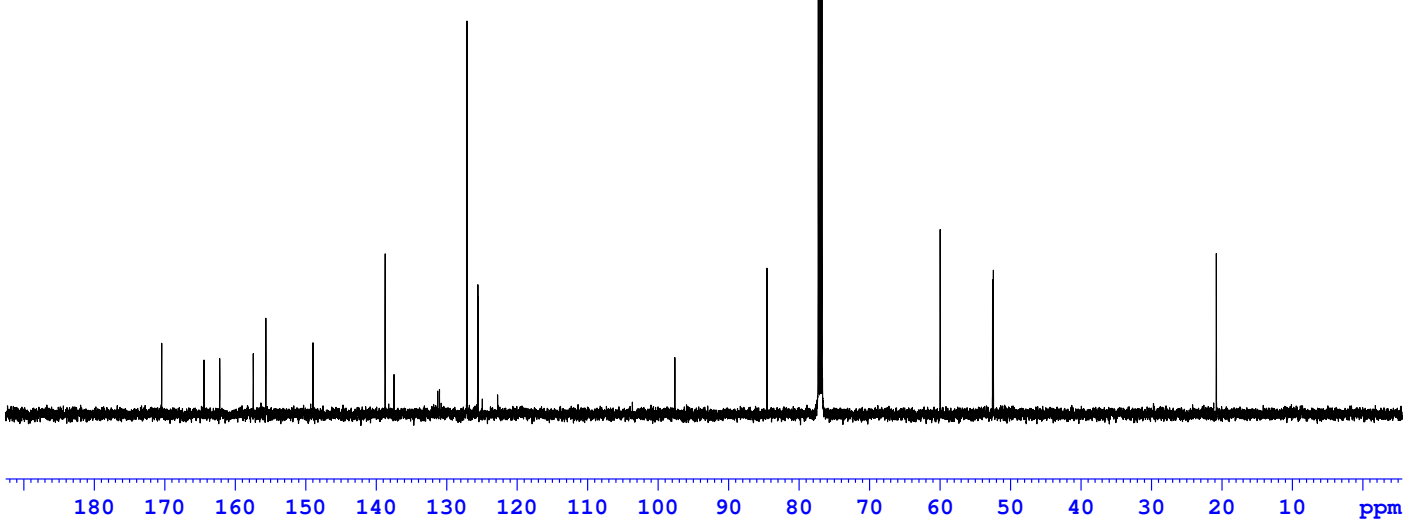




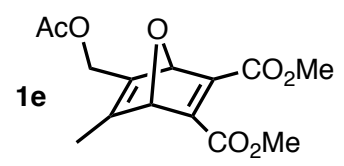

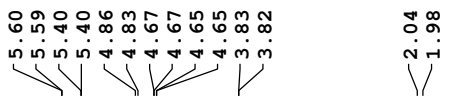

$500 \mathrm{~Hz}$

Solvent: $\mathrm{CDCl}_{3}$
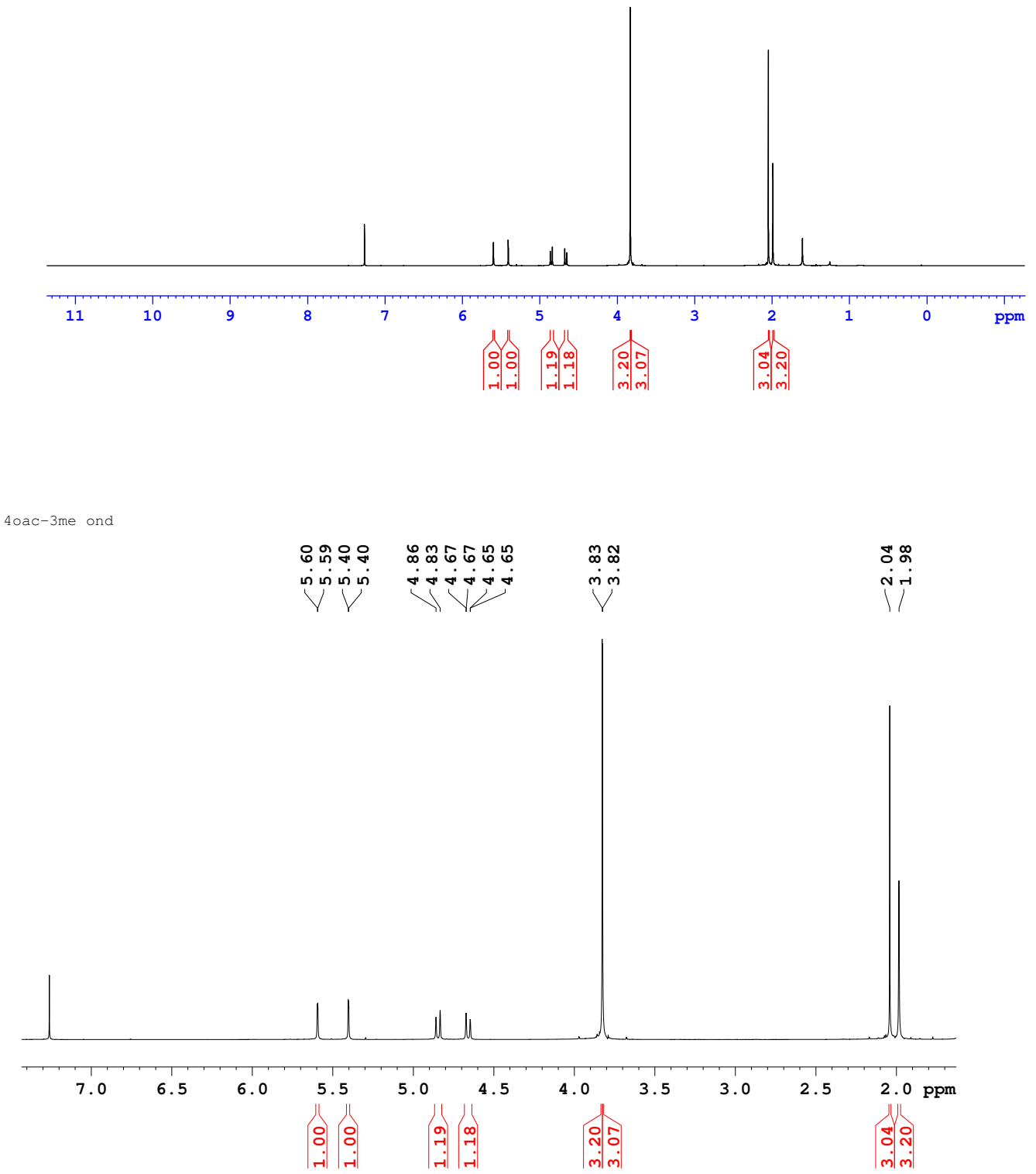


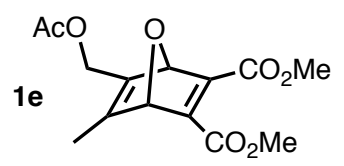

4-oac-3-me ond

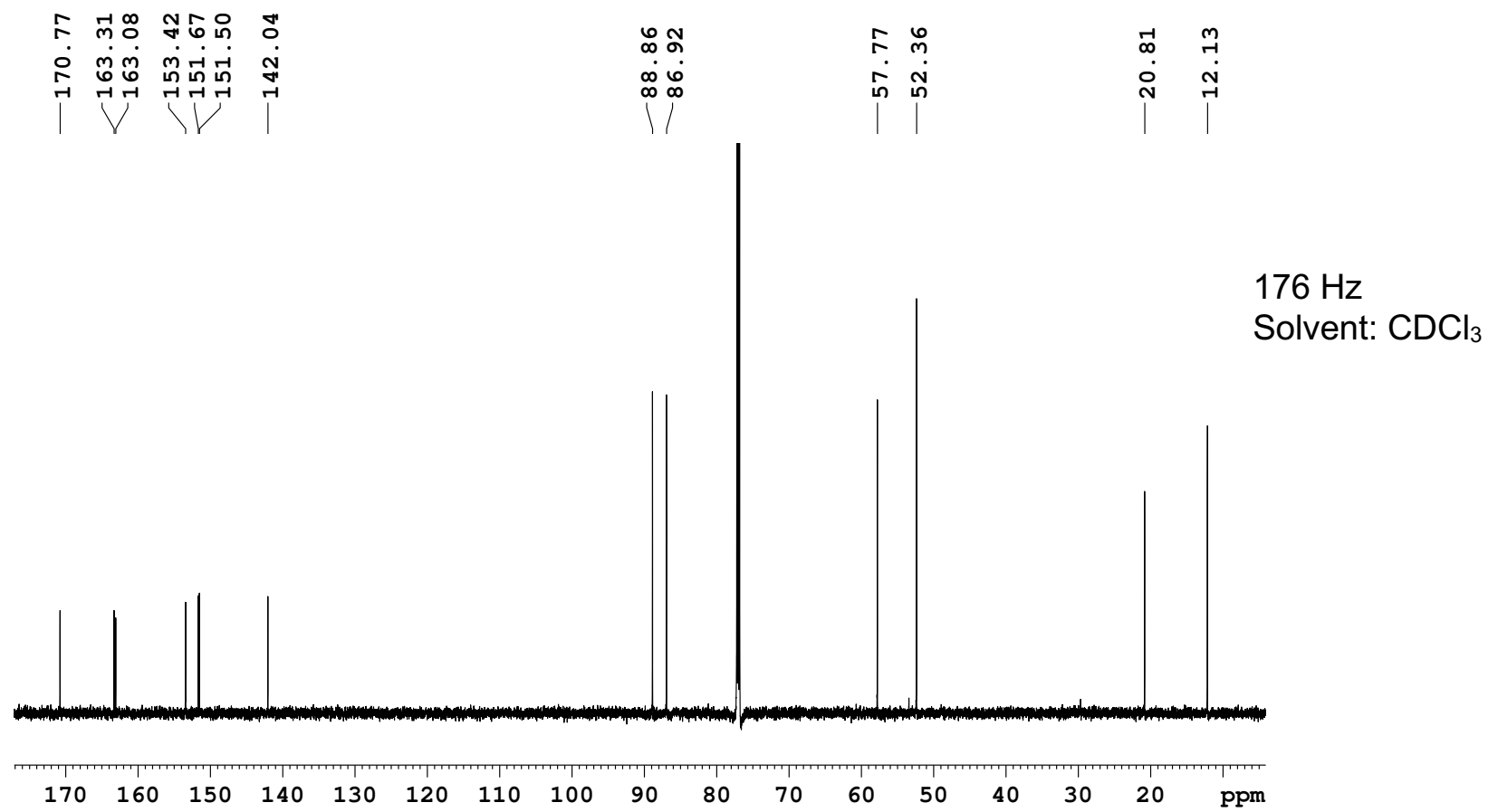



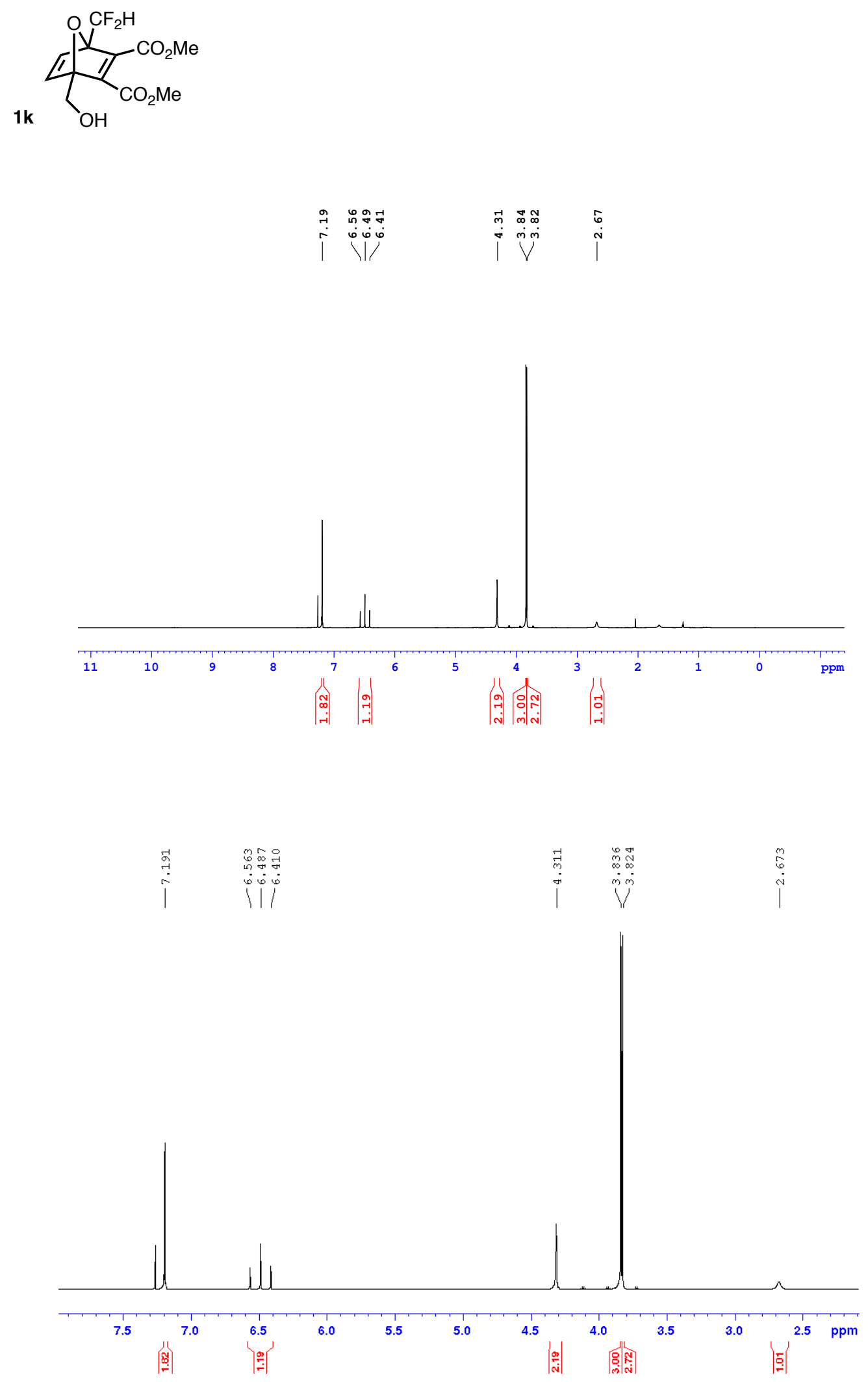

$700 \mathrm{~Hz}$

Solvent: $\mathrm{CDCl}_{3}$ 
<smiles>COC(=O)C1=C(CO)C2(CO)C=CC1(C(F)F)O2</smiles>

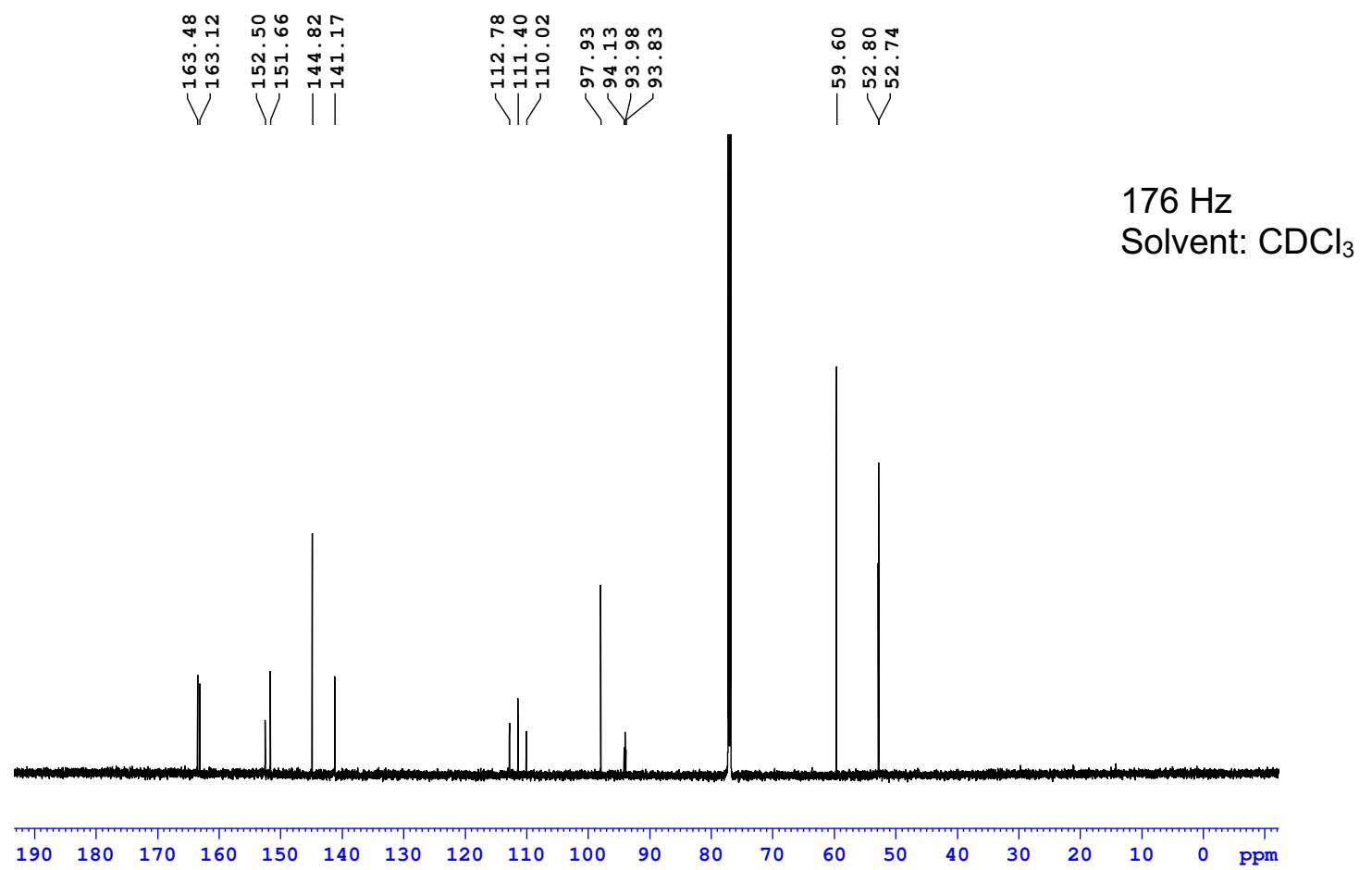



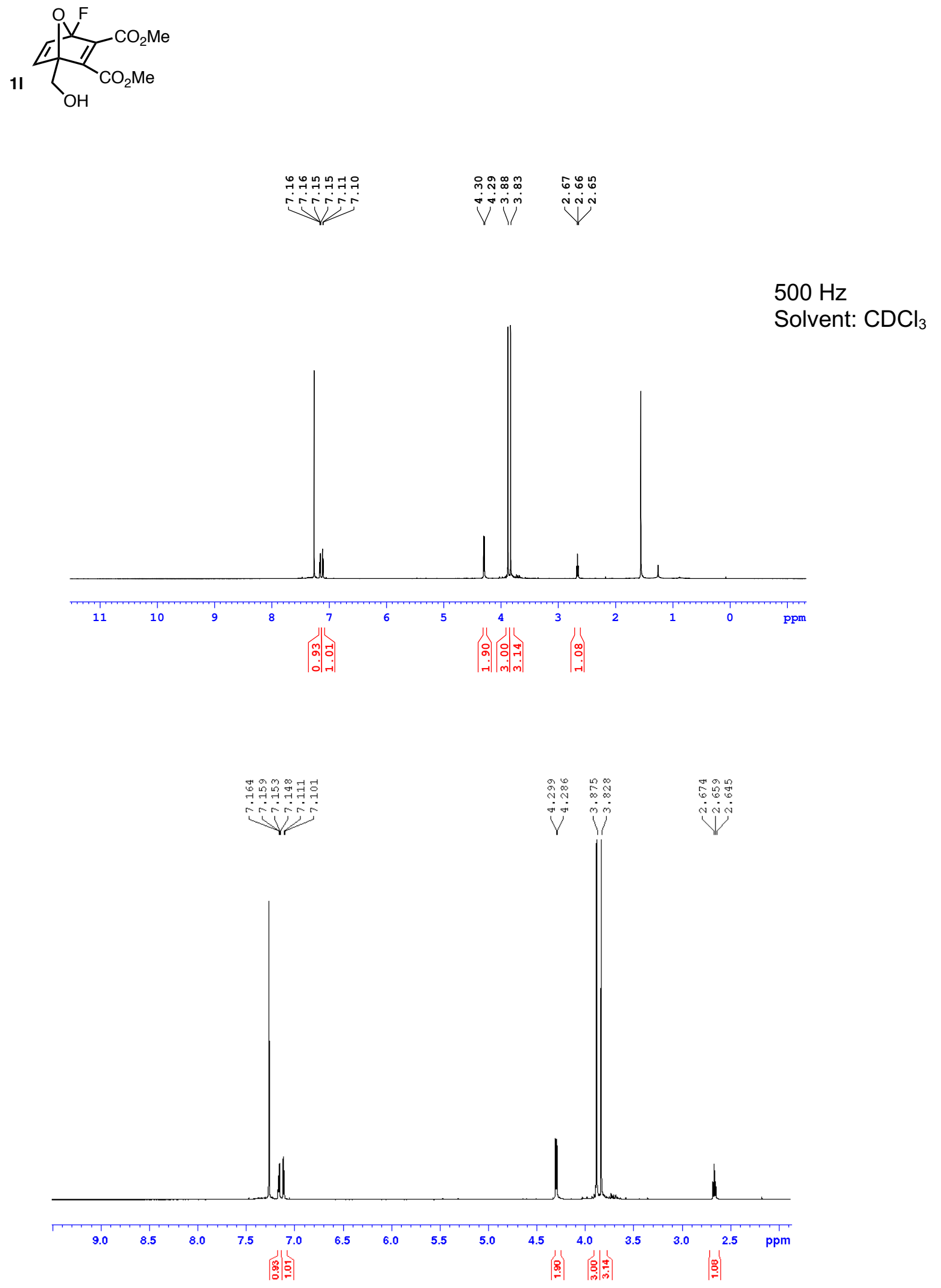

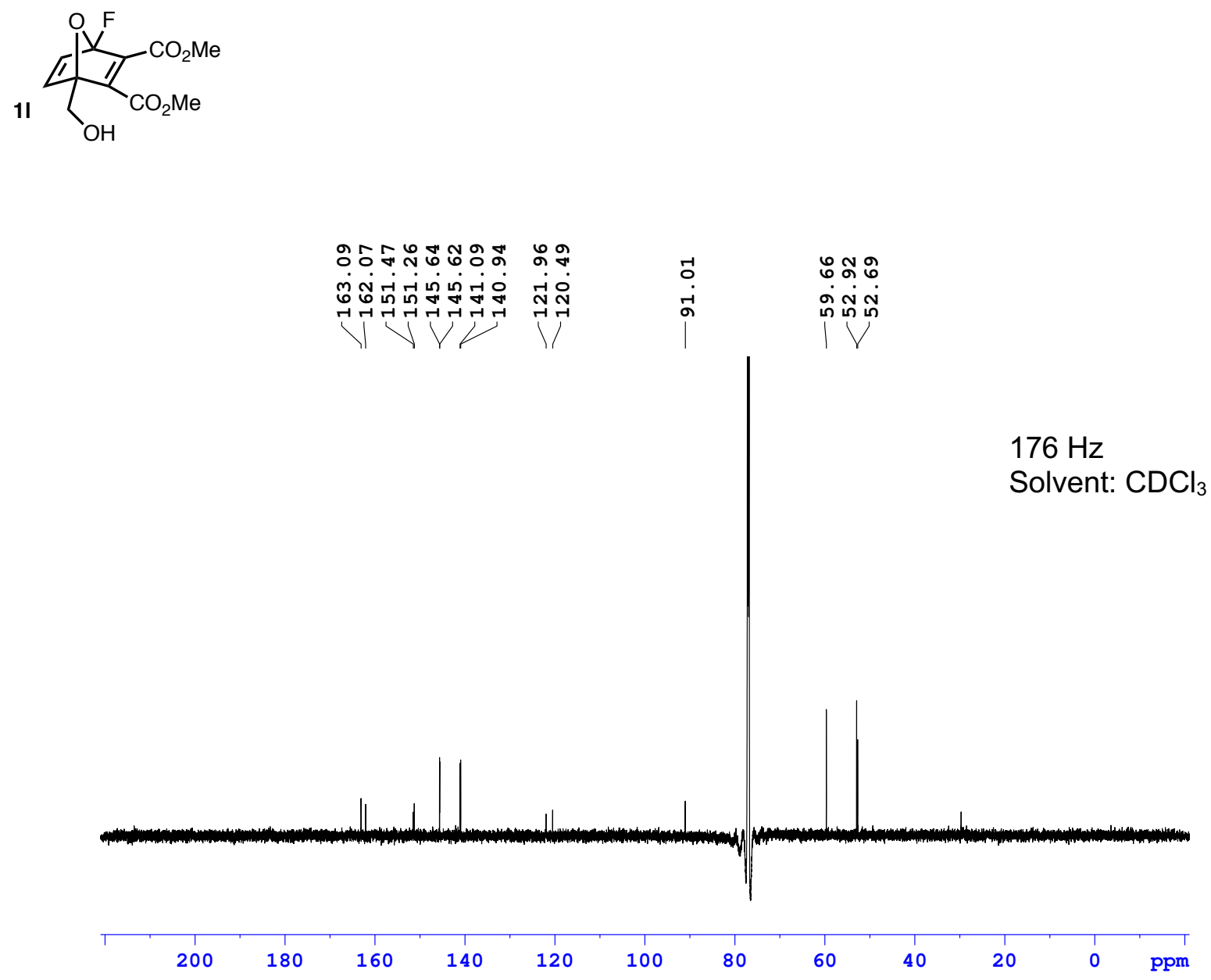

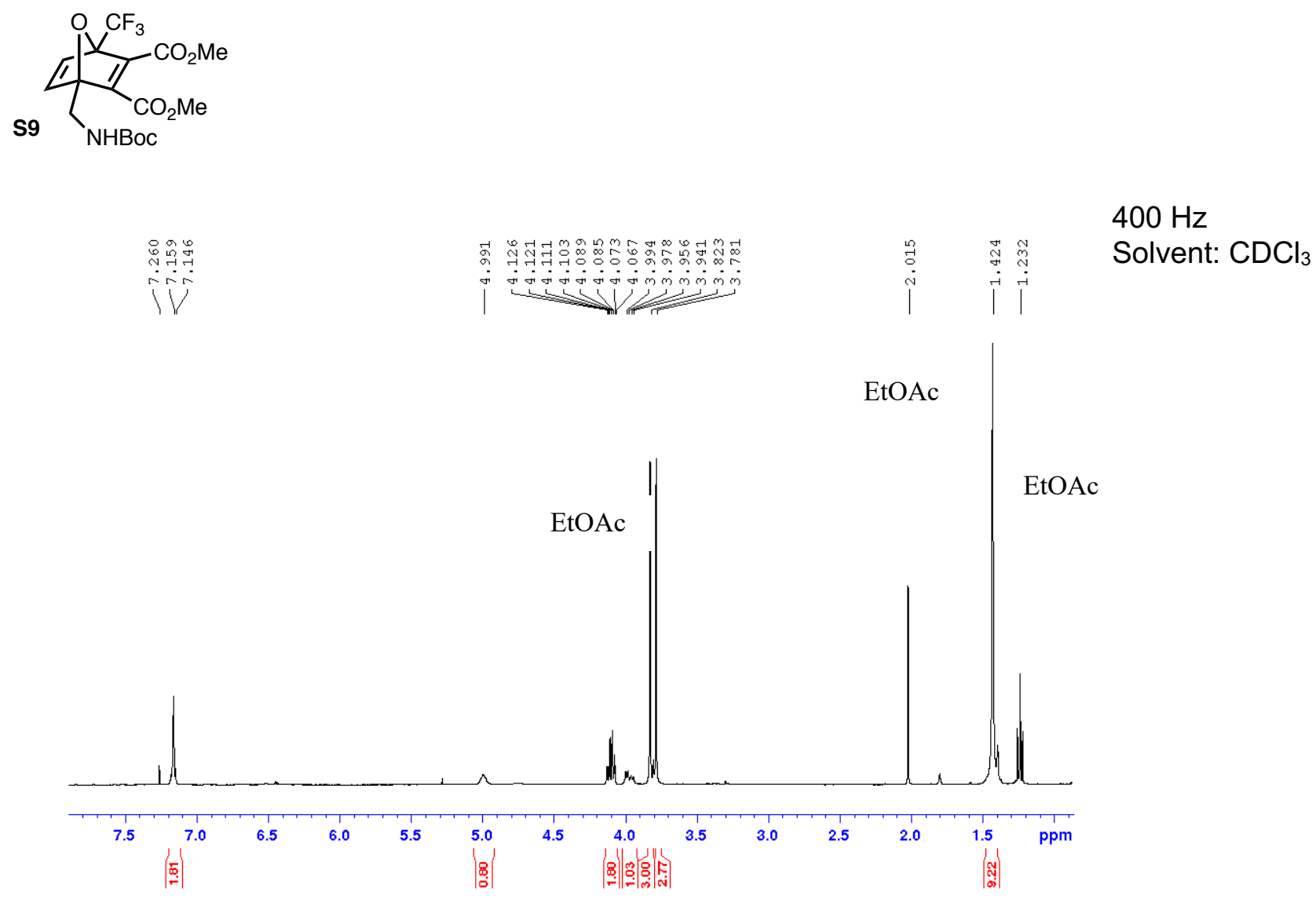

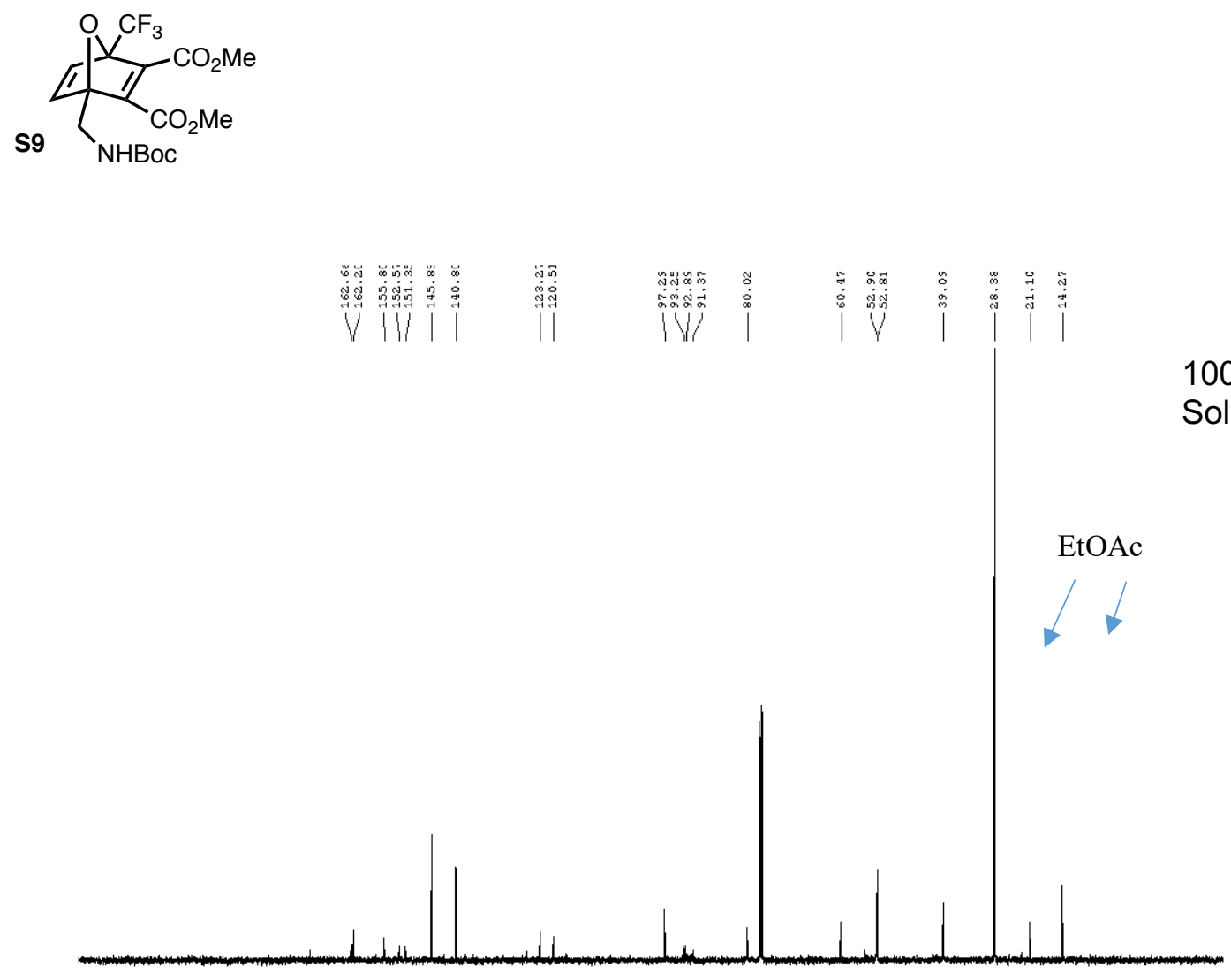

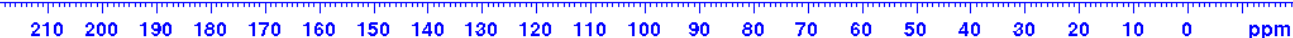




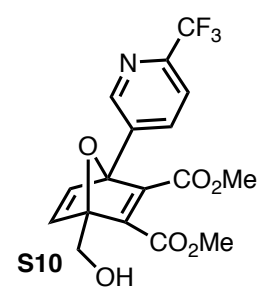

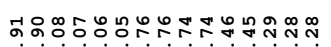

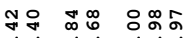

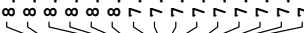

रु

$400 \mathrm{~Hz}$

Solvent: $\mathrm{CDCl}_{3}$
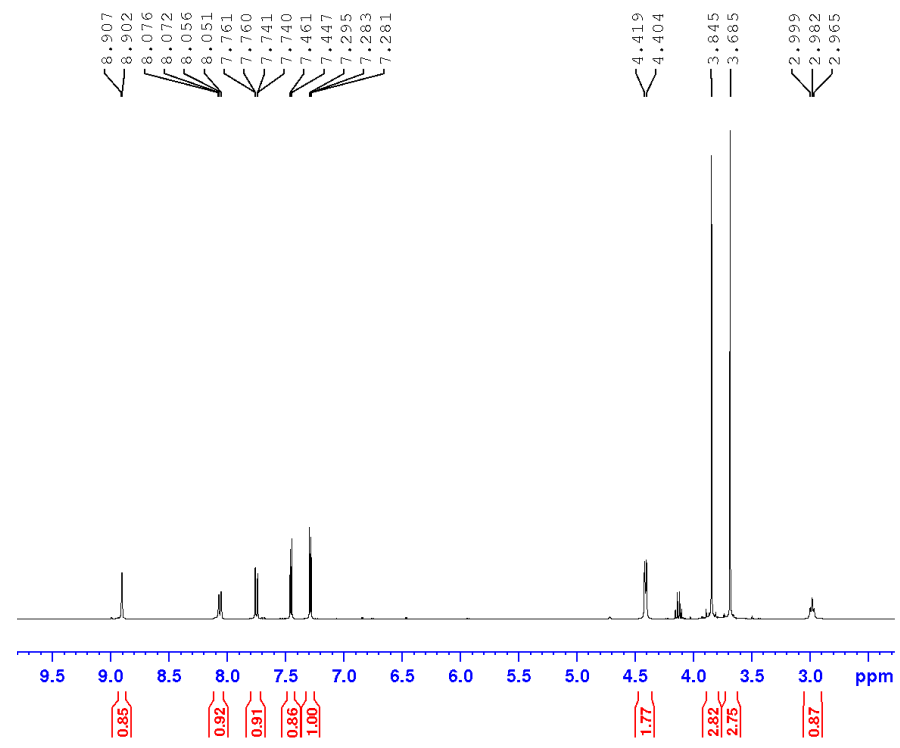

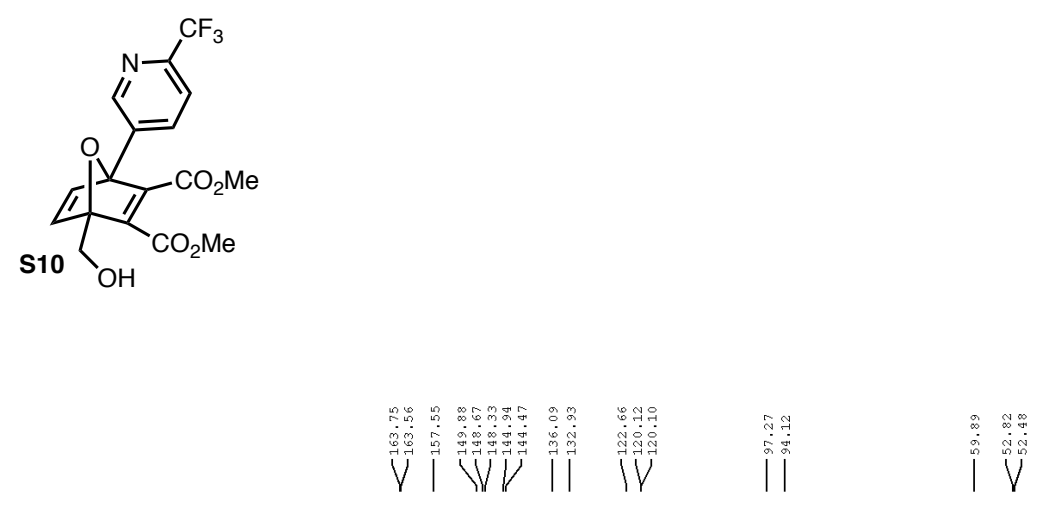

$100 \mathrm{~Hz}$

Solvent: $\mathrm{CDCl}_{3}$

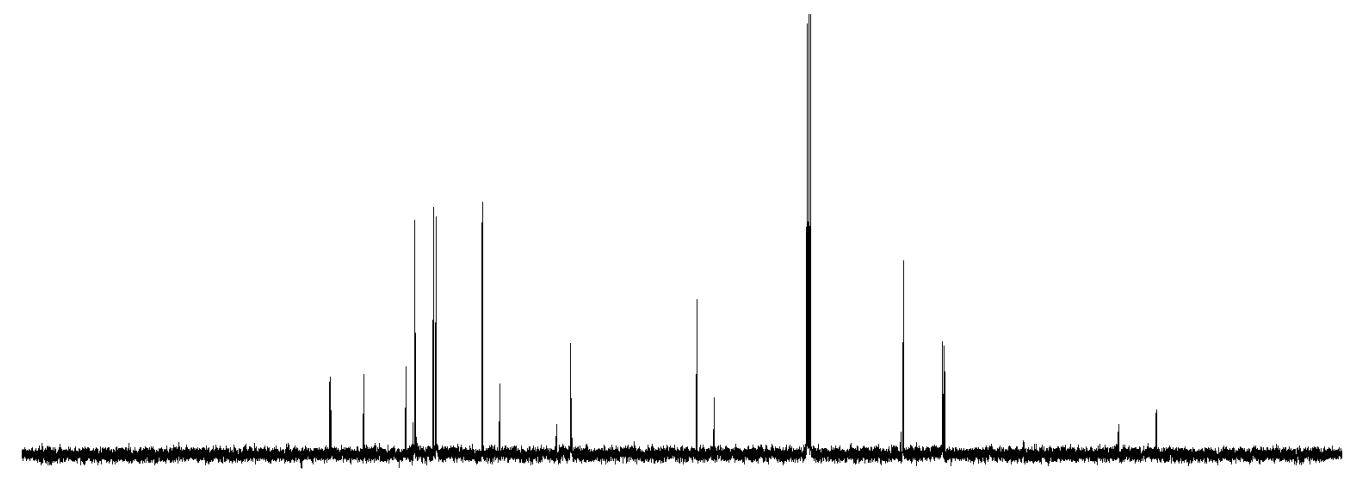

$\begin{array}{llllllllllllllllllllll}210 & 200 & 190 & 180 & 170 & 160 & 150 & 140 & 130 & 120 & 110 & 100 & 90 & 80 & 70 & 60 & 50 & 40 & 30 & 20 & 10 & 0\end{array}$ 

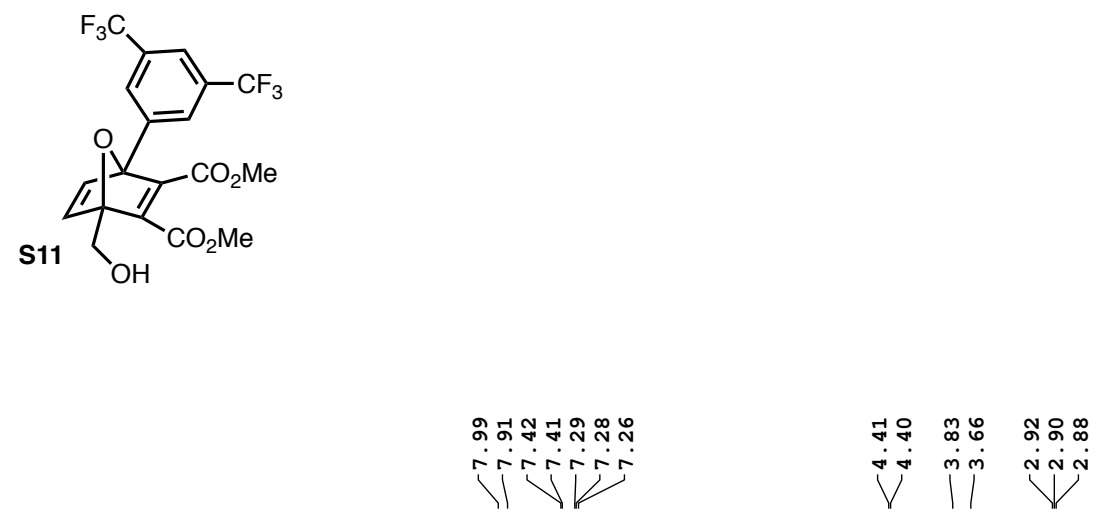

$400 \mathrm{~Hz}$

Solvent: $\mathrm{CDCl}_{3}$

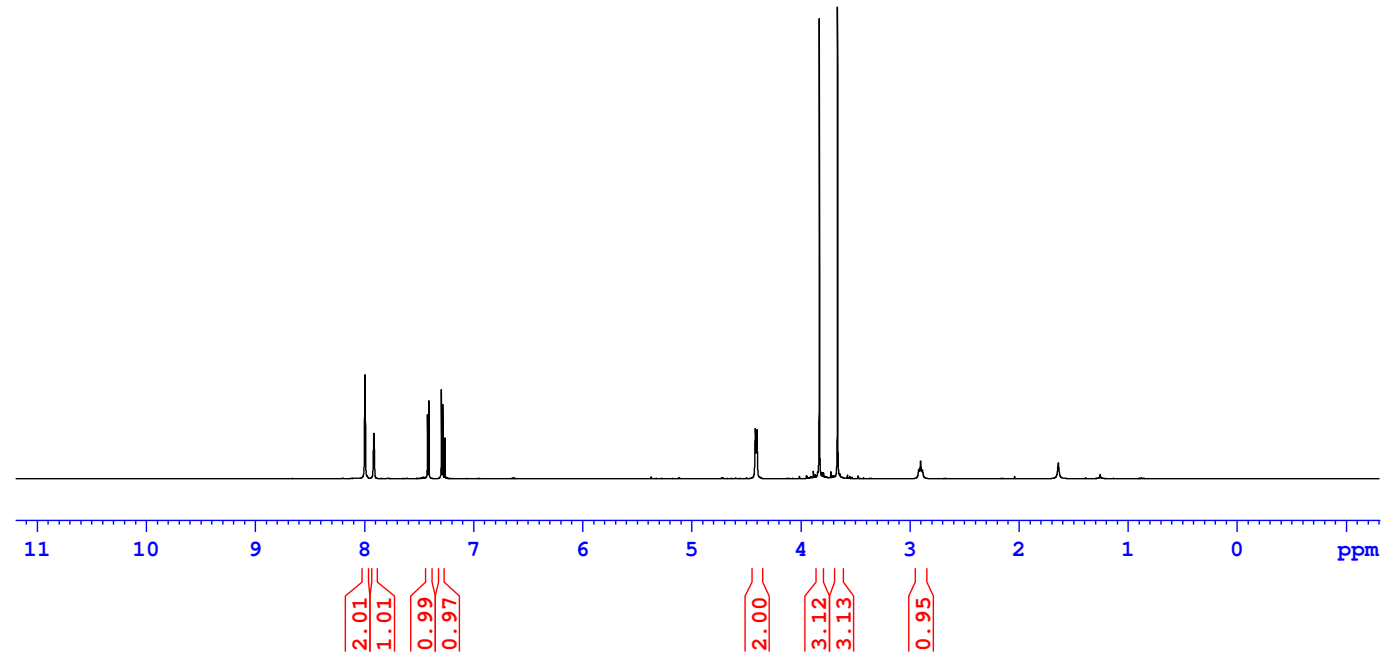



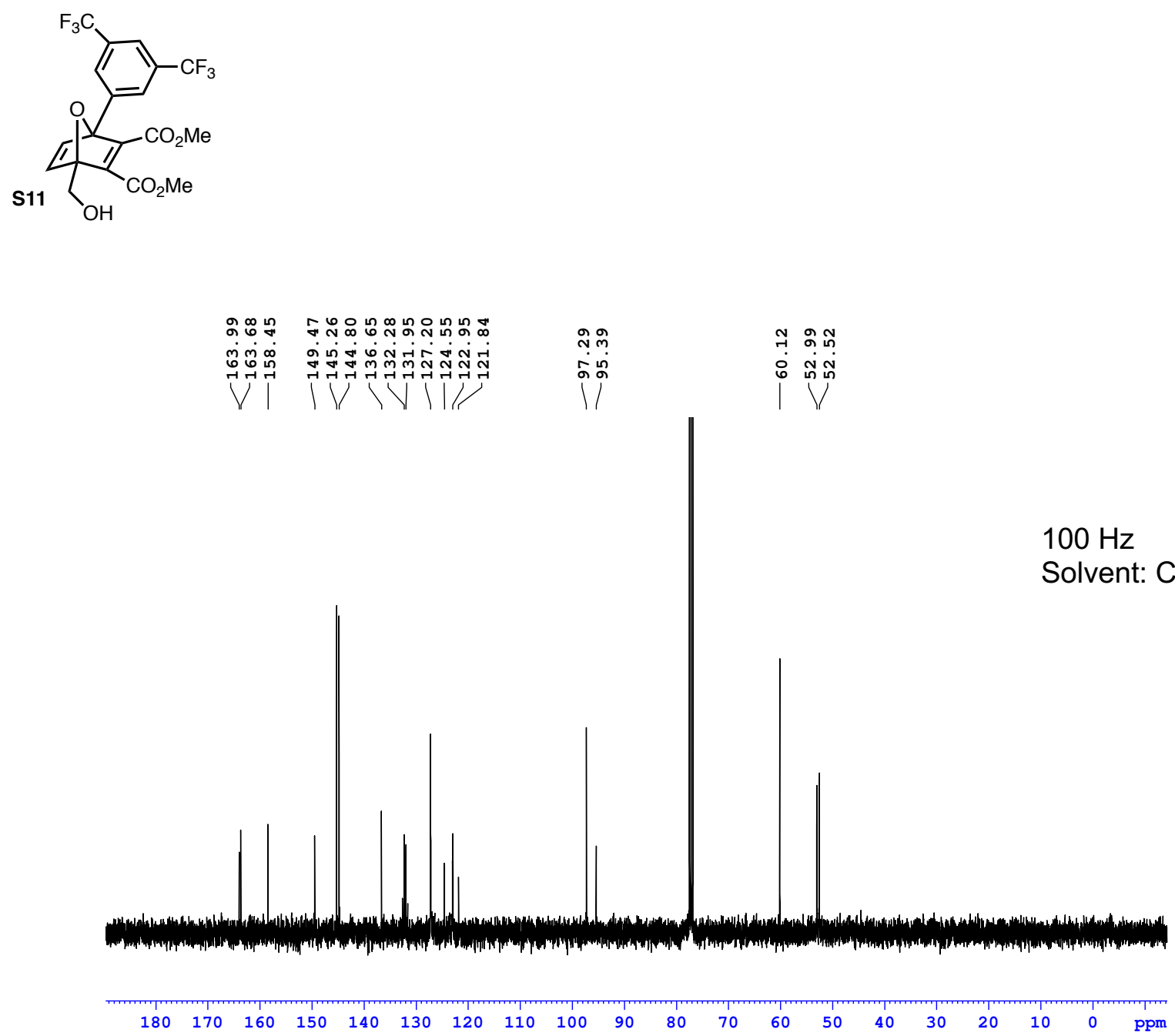

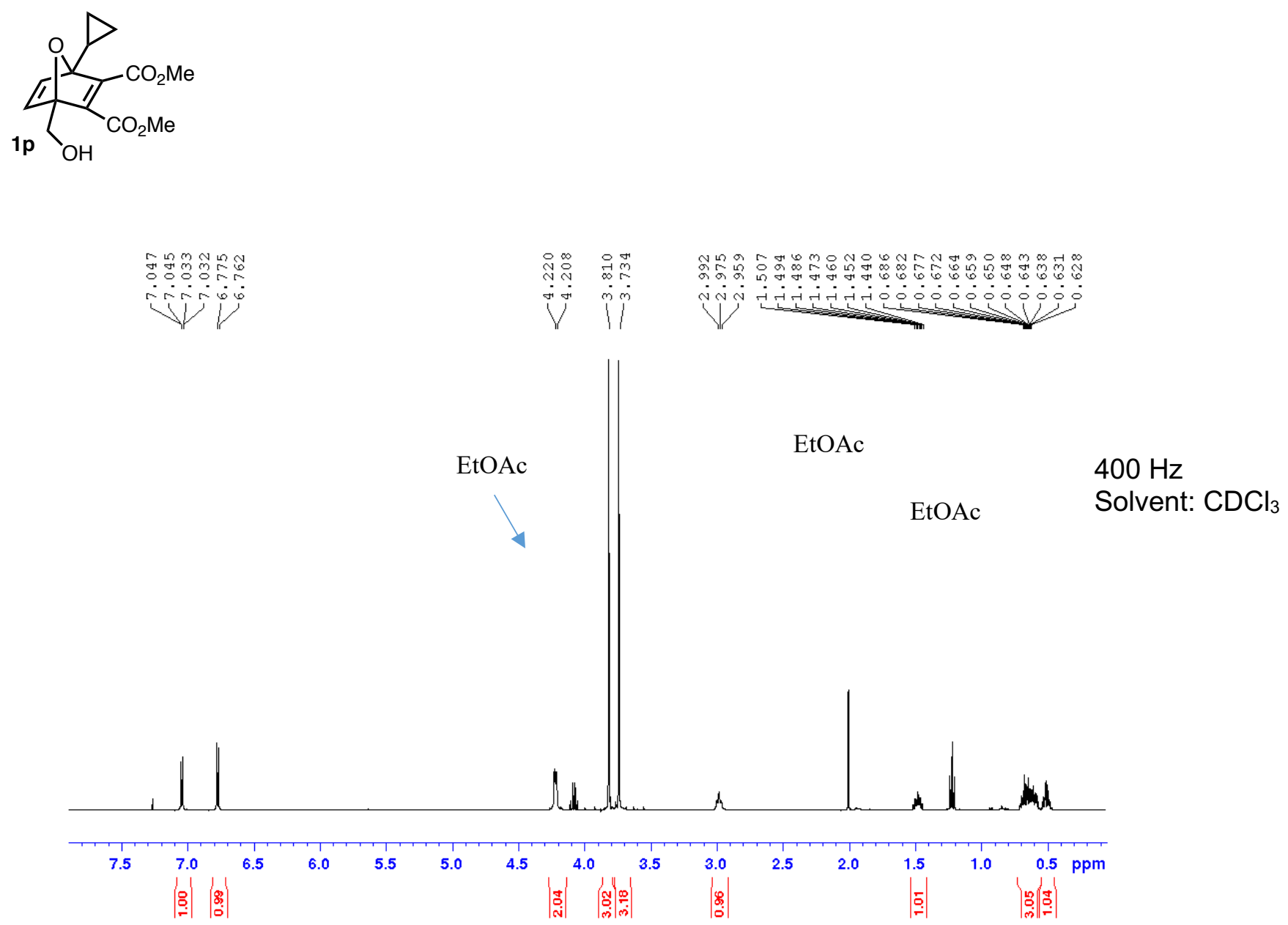

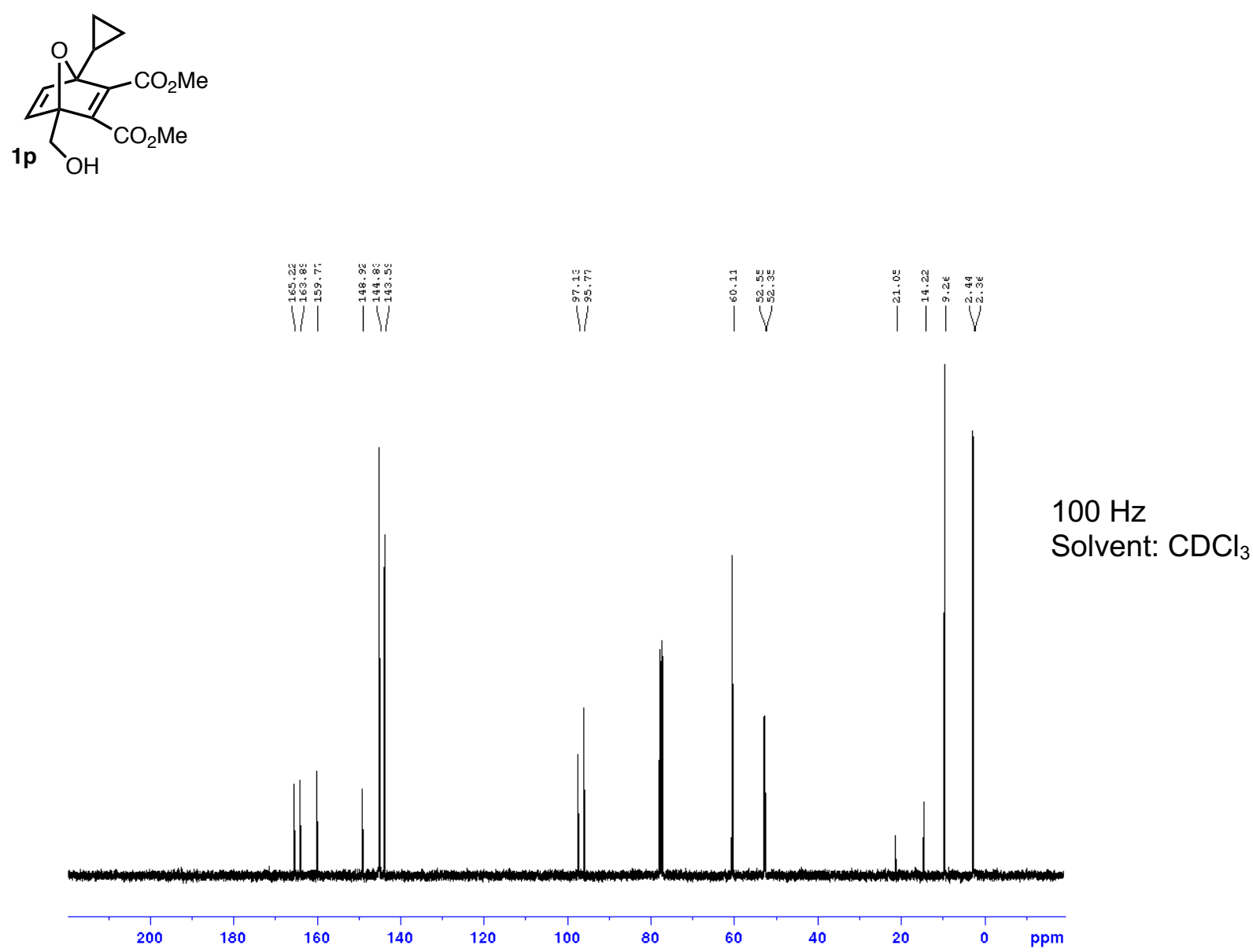

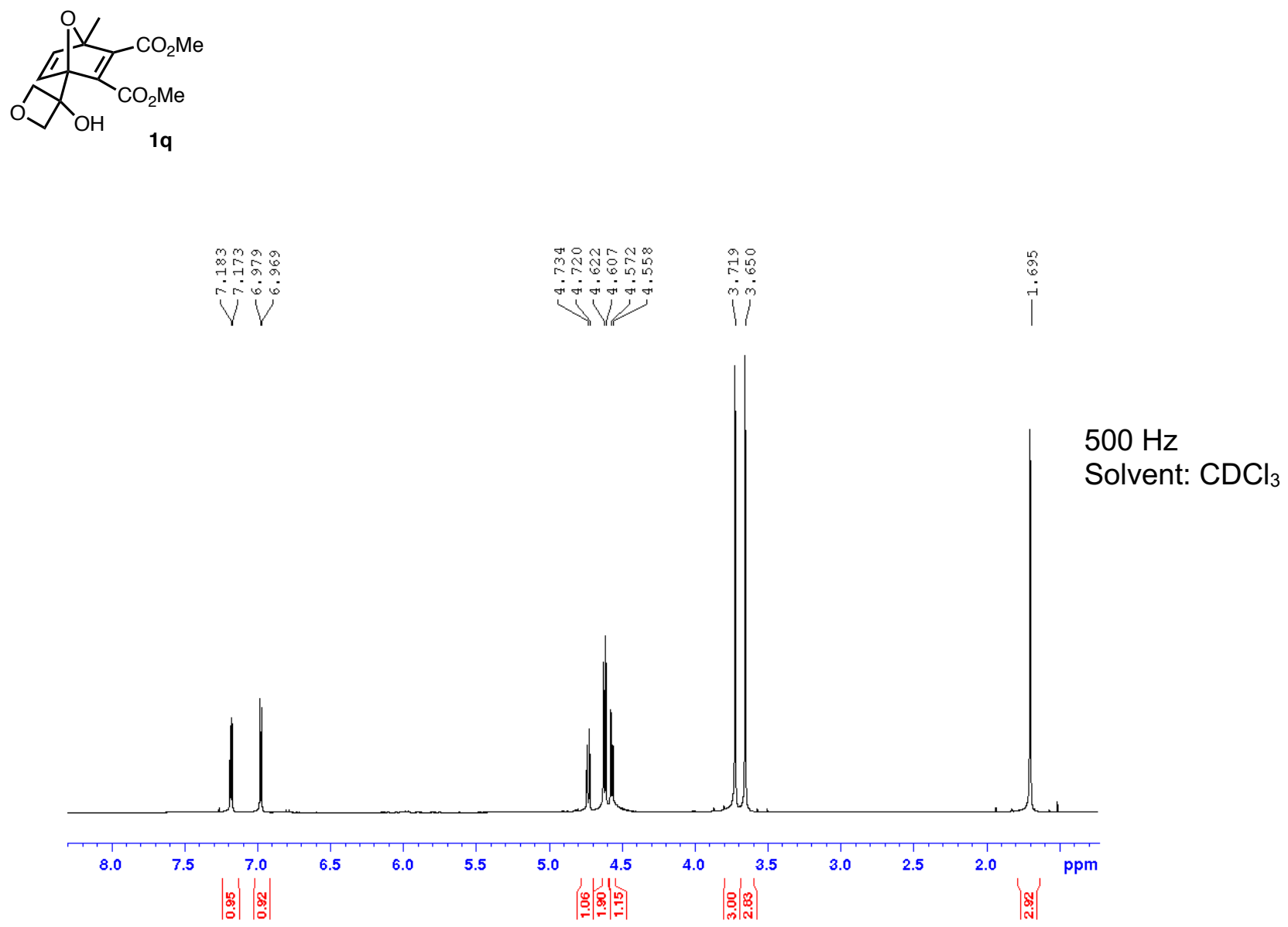

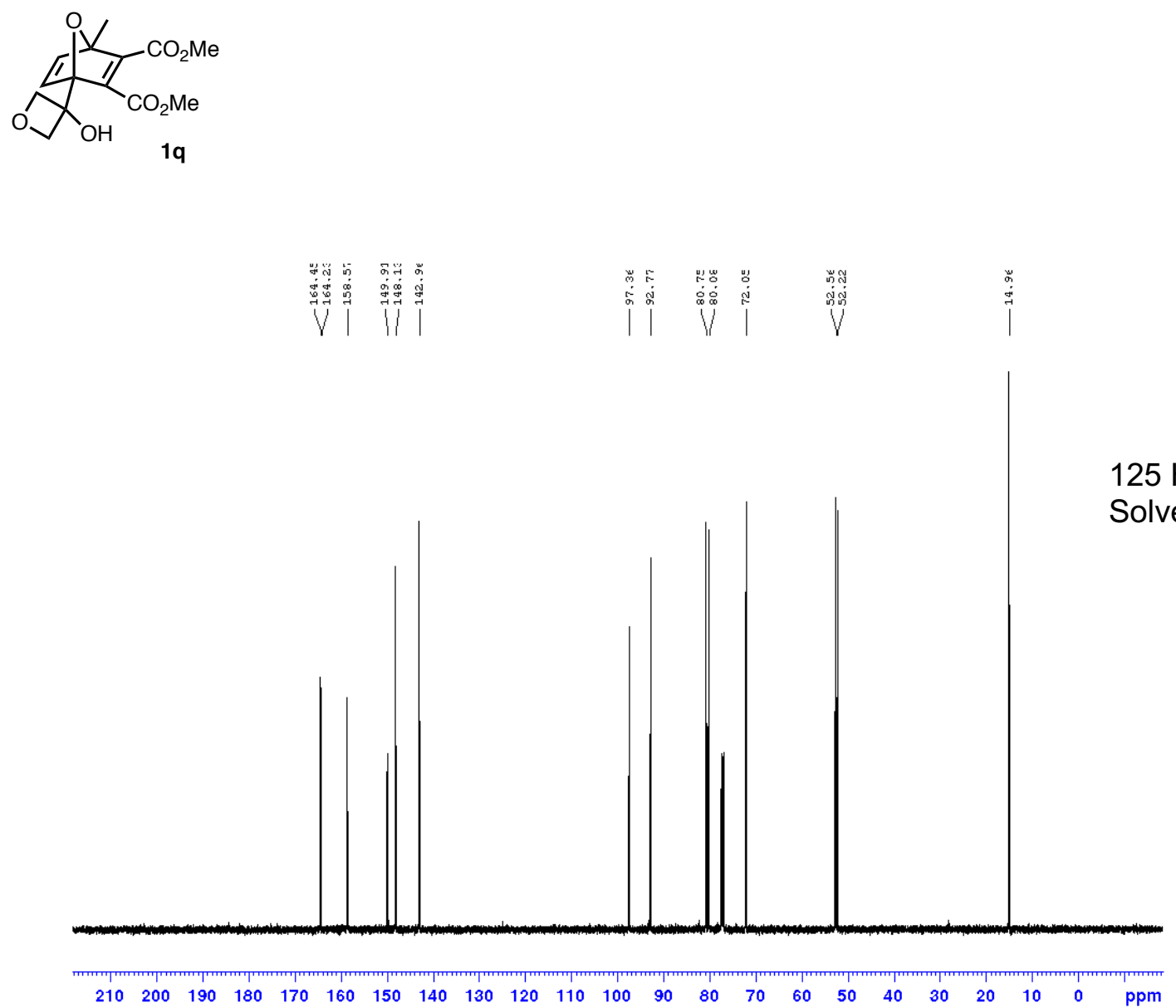


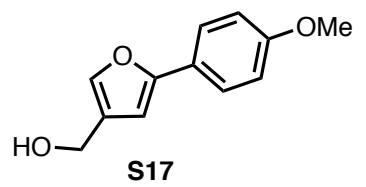

furan-2phome-4oh

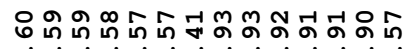
rararabogo
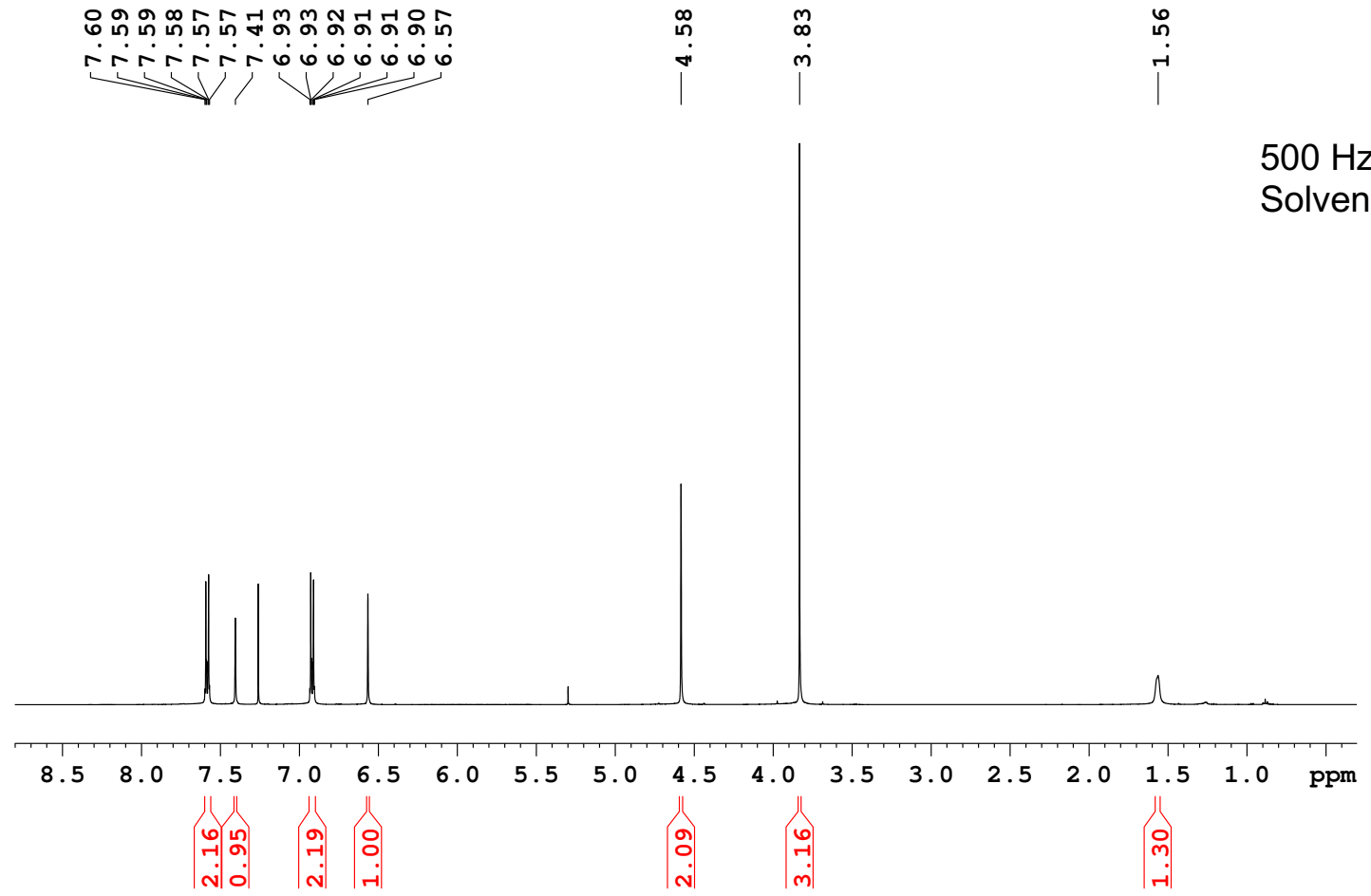

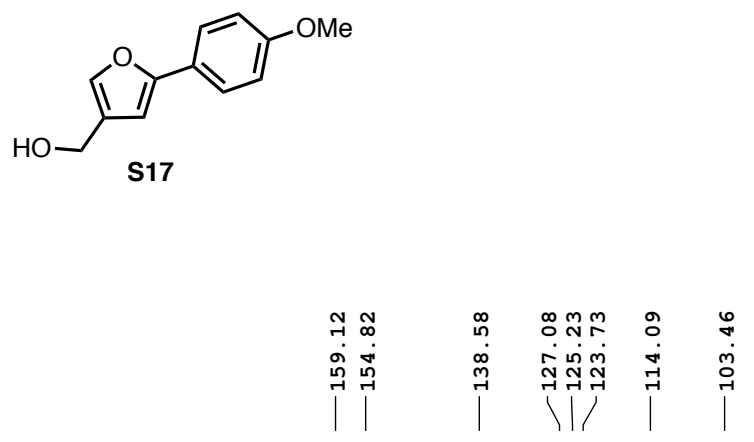

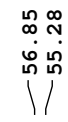

$125 \mathrm{~Hz}$

Solvent: $\mathrm{CDCl}_{3}$

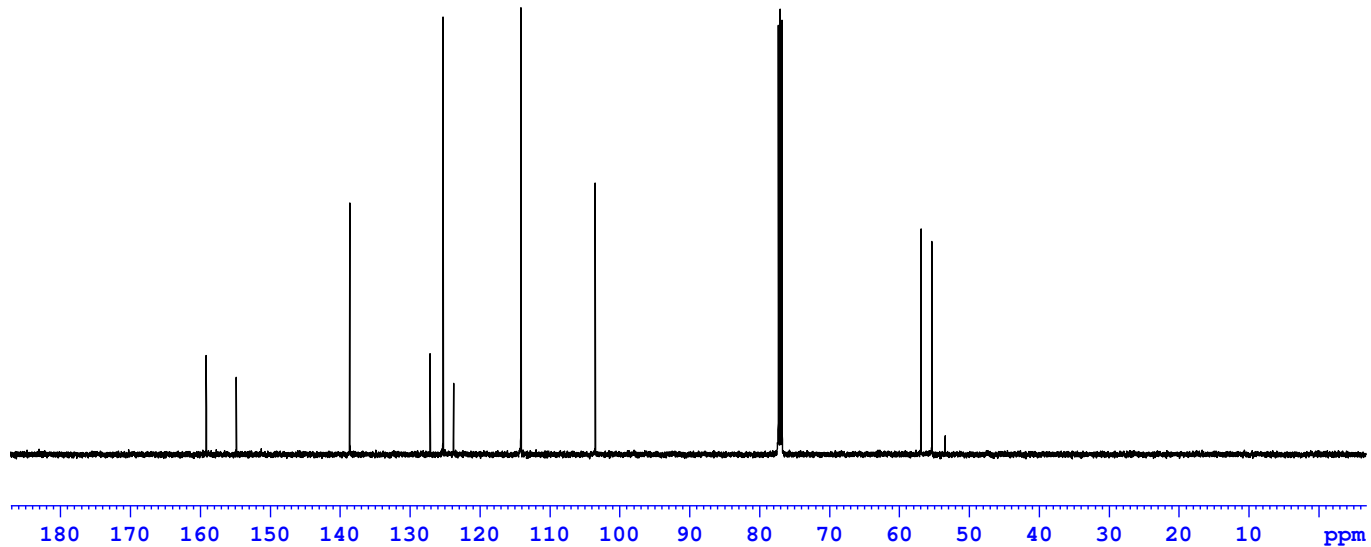



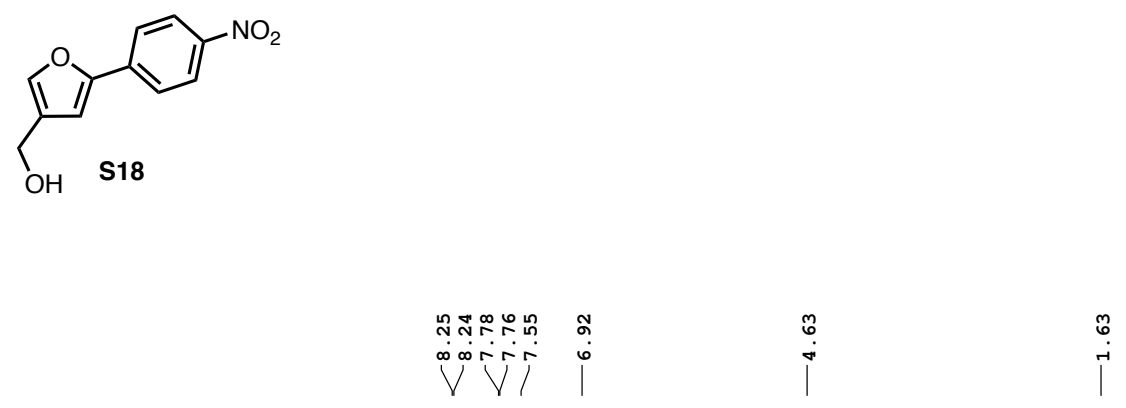

$500 \mathrm{~Hz}$

Solvent: $\mathrm{CDCl}_{3}$

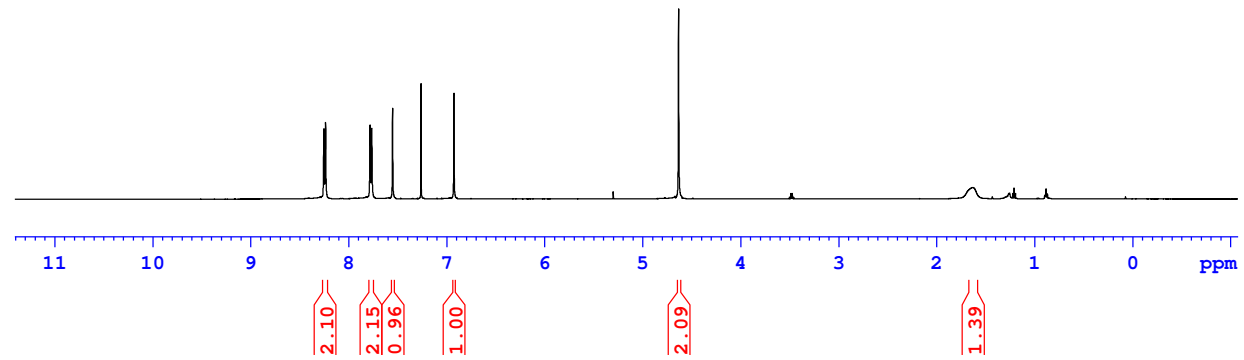

furan-2phno2-4oh

ஸู๊

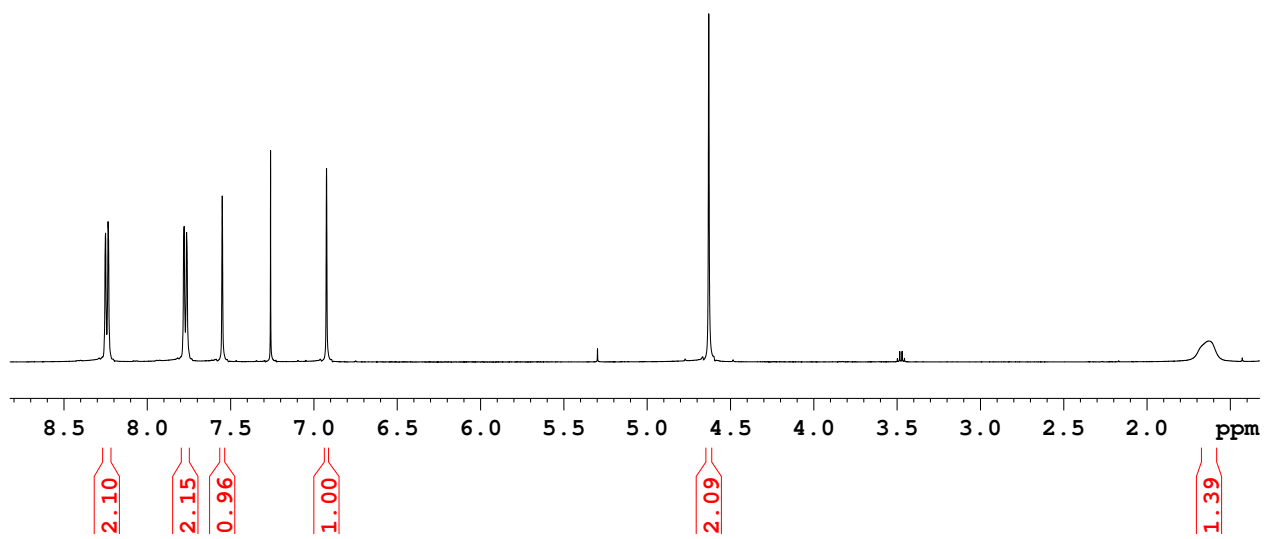



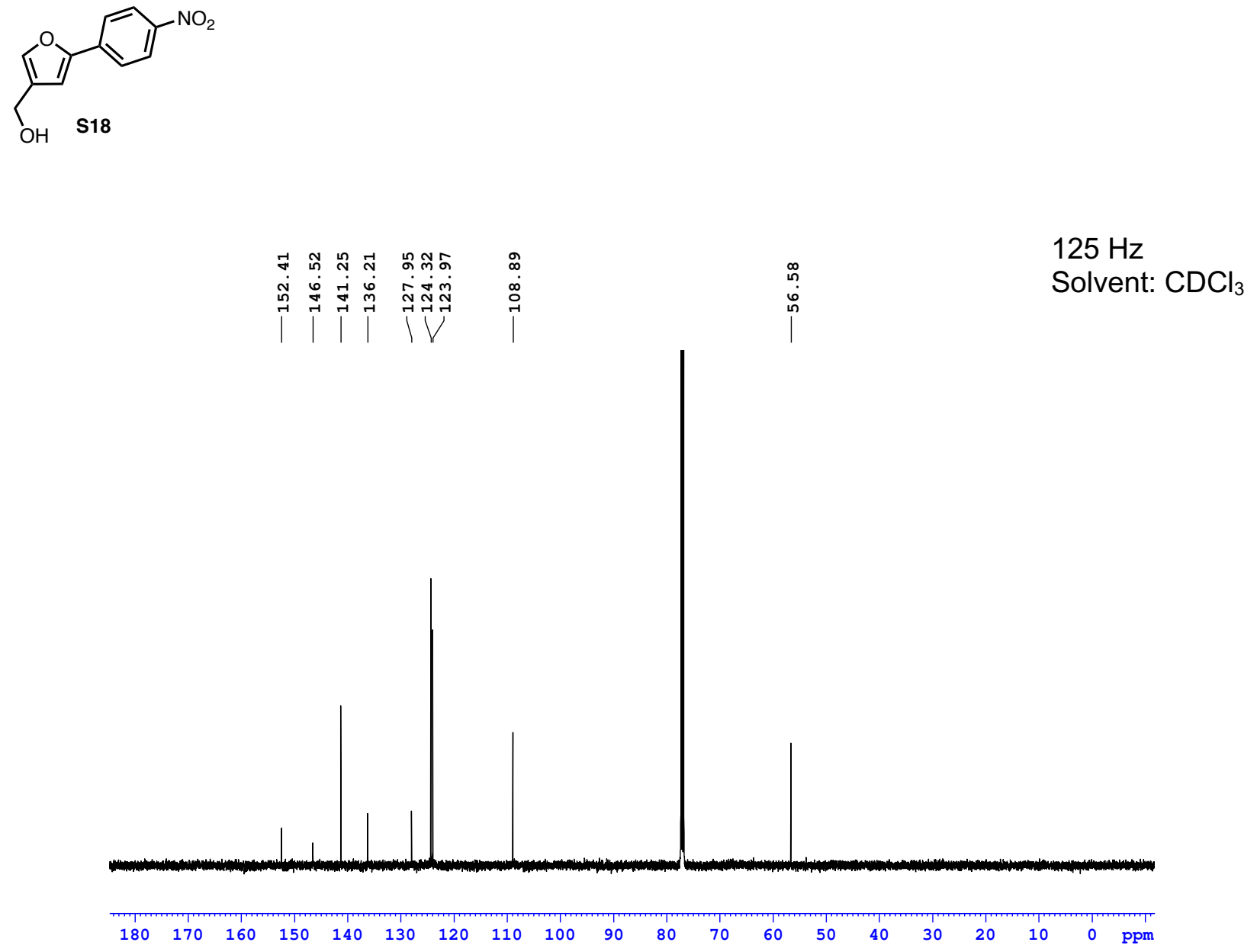


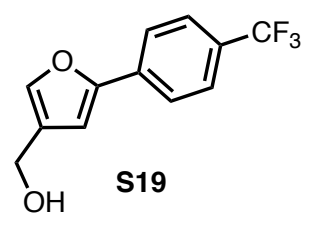

furan-2ph-cf $3-4 o h$

조ำ유 क्ष

irisi

6

i

$\stackrel{\text { in }}{i}$

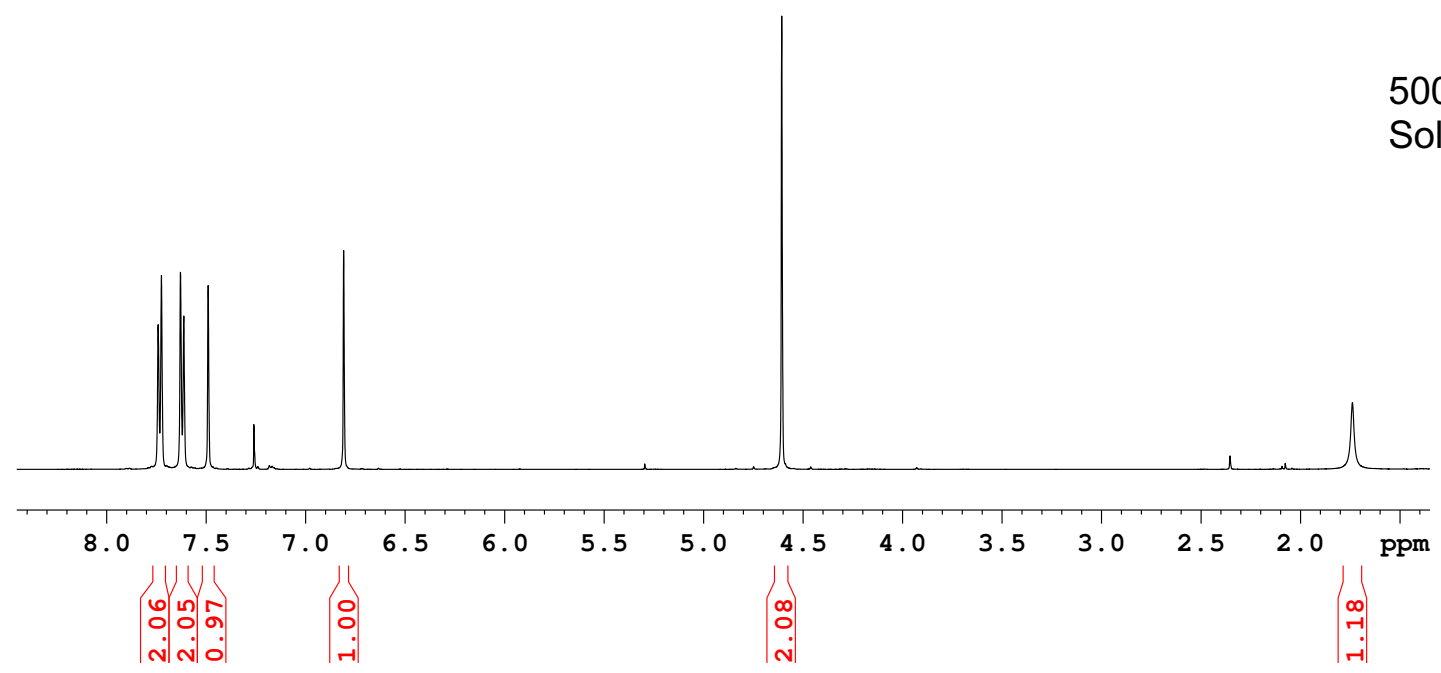

$500 \mathrm{~Hz}$

Solvent: $\mathrm{CDCl}_{3}$ 

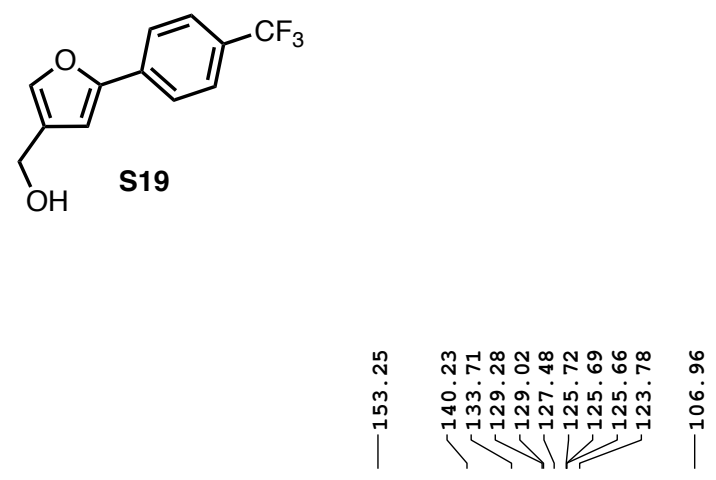

$125 \mathrm{~Hz}$

Solvent: $\mathrm{CDCl}_{3}$

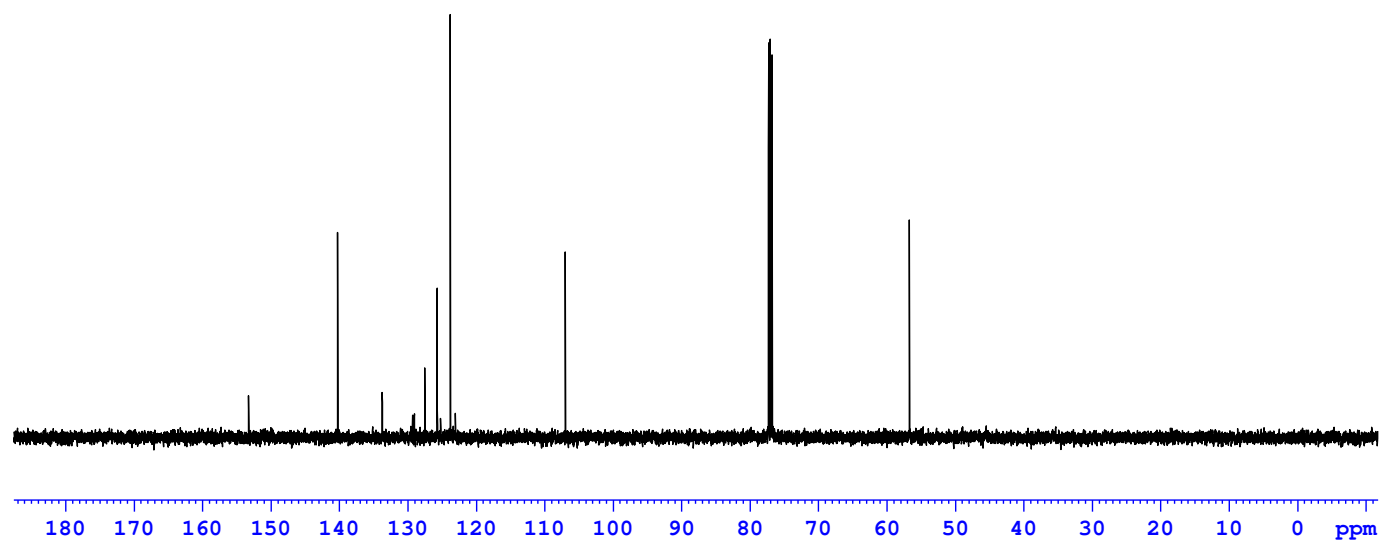




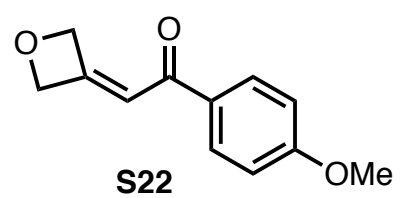

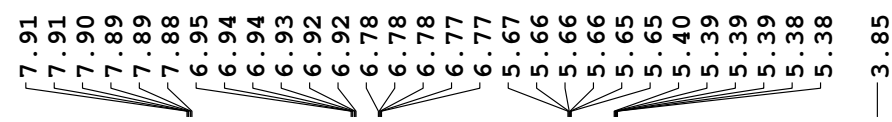

$500 \mathrm{~Hz}$

Solvent: $\mathrm{CDCl}_{3}$

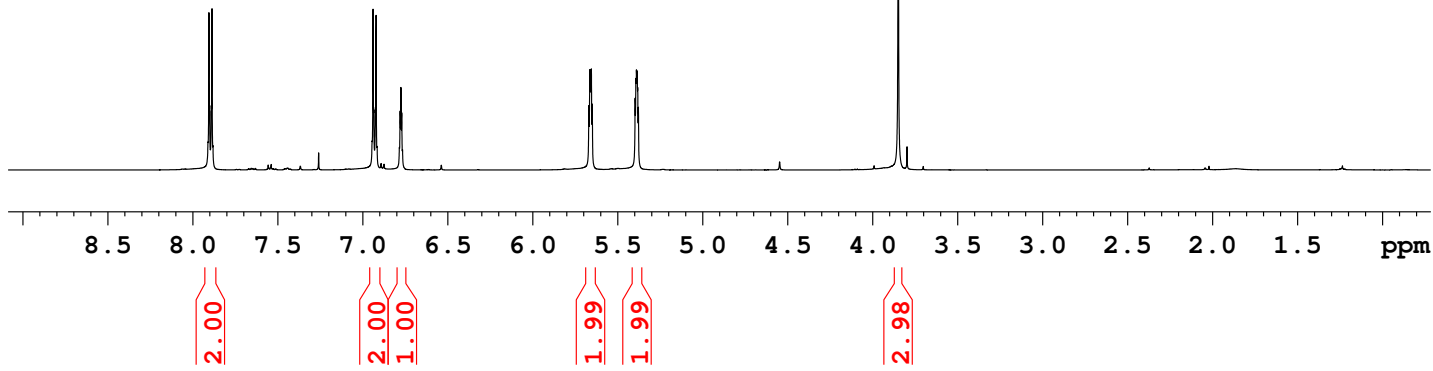




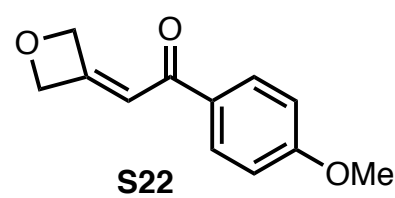

|

$\mid$

$125 \mathrm{~Hz}$

Solvent: $\mathrm{CDCl}_{3}$
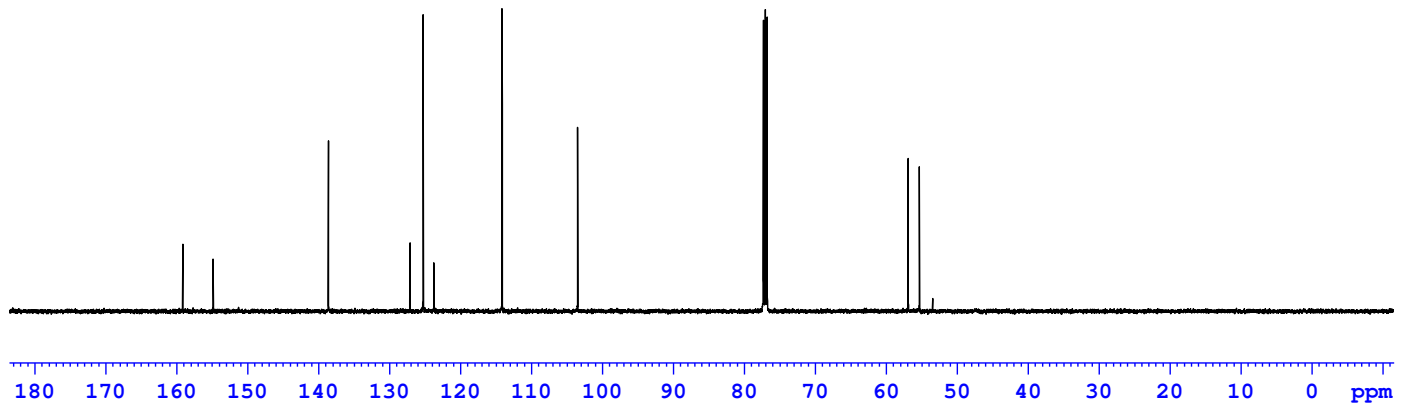


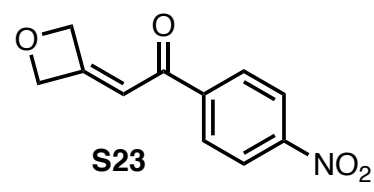

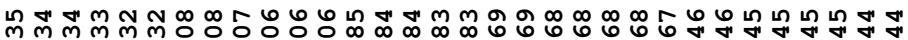

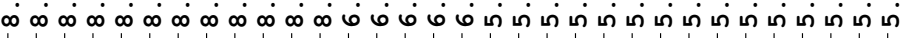

$\longrightarrow 1$

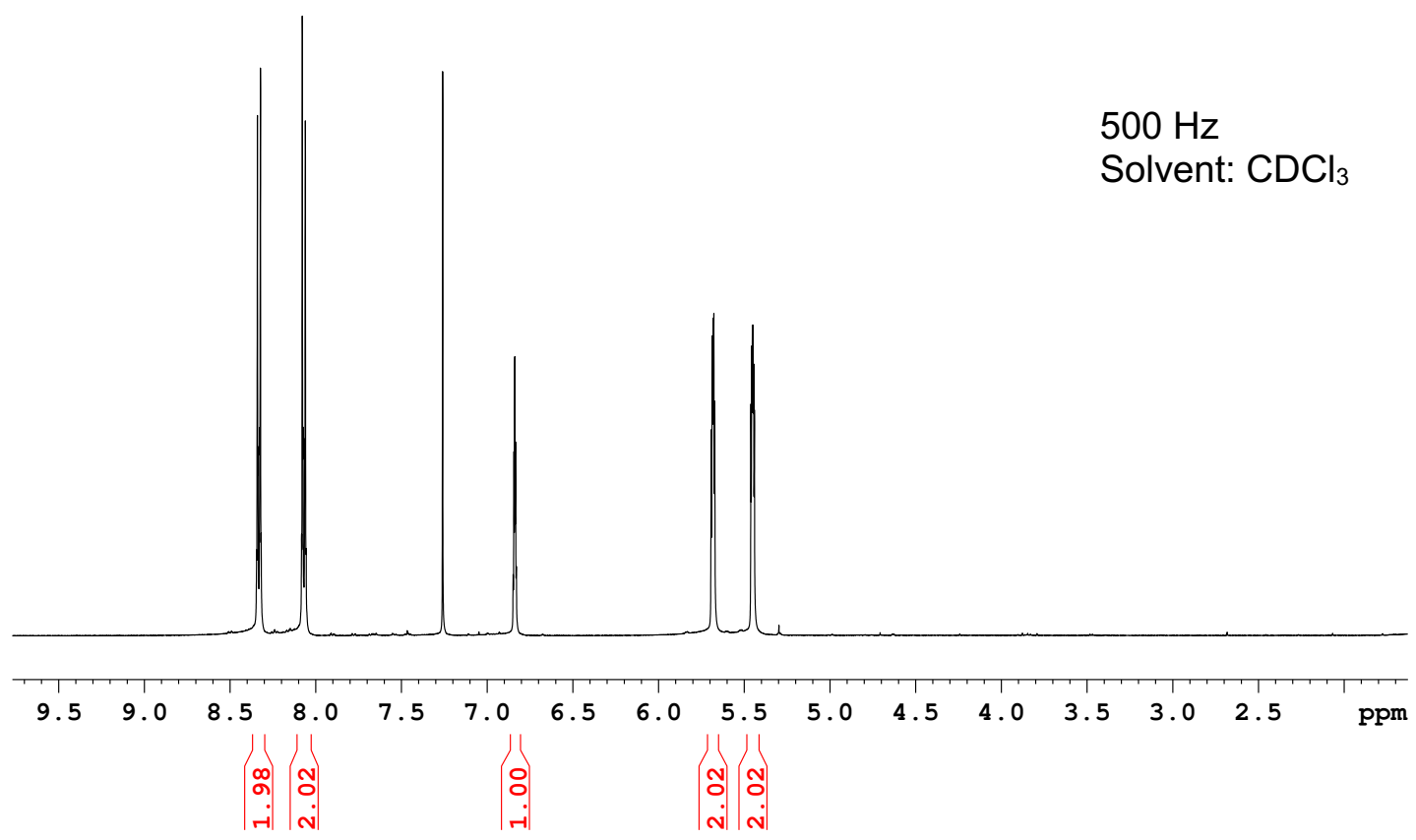




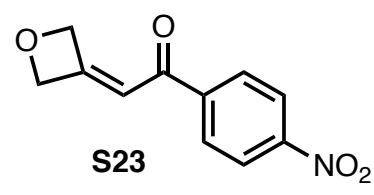

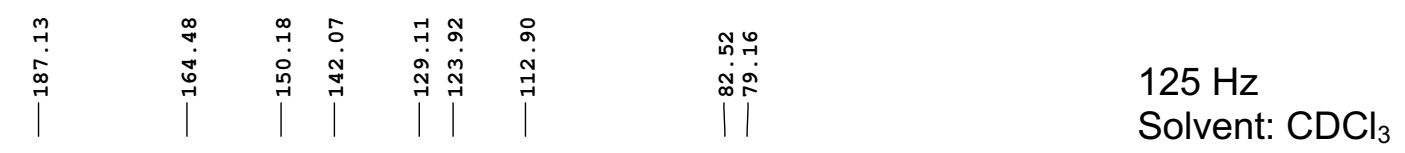

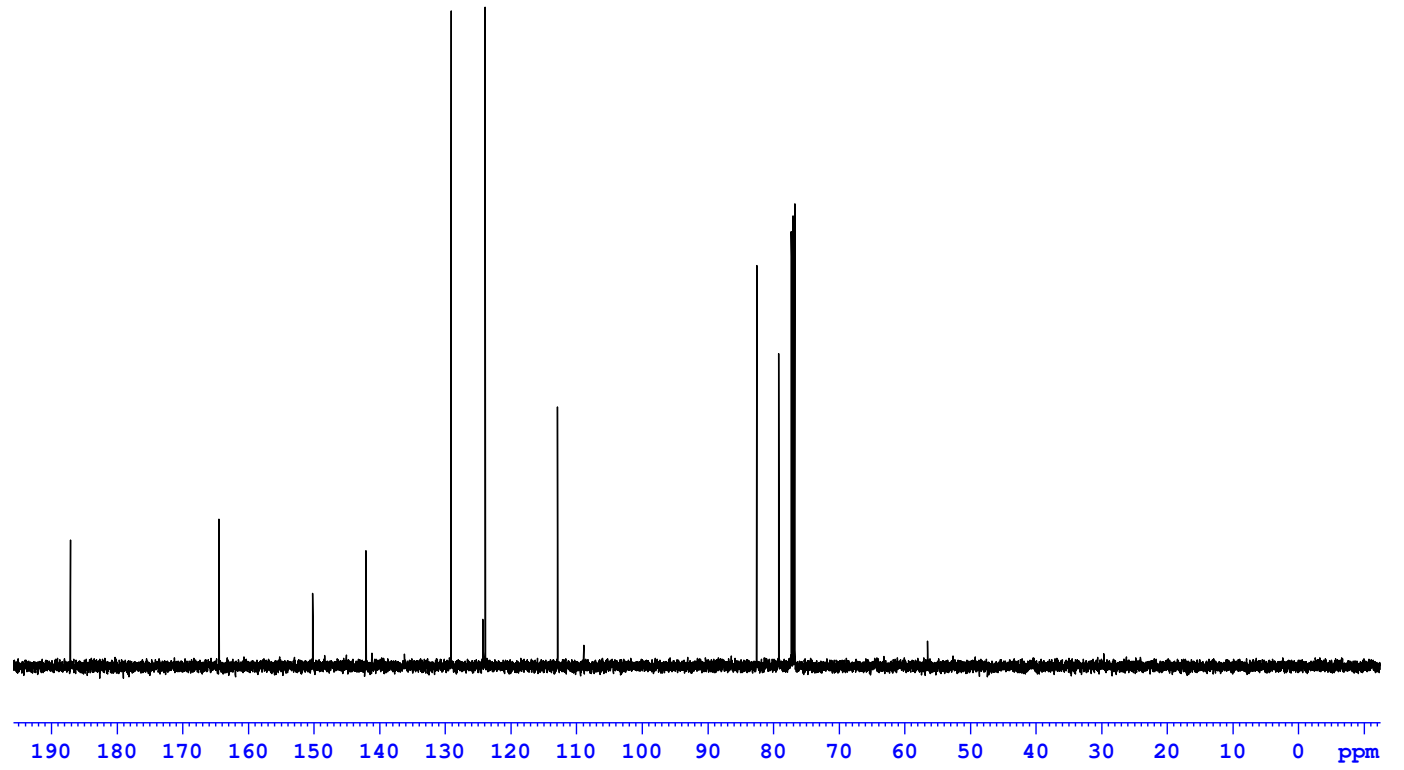




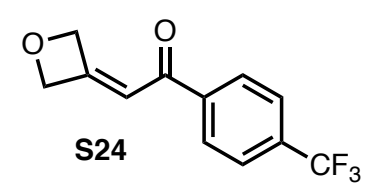

$500 \mathrm{~Hz}$

Solvent: $\mathrm{CDCl}_{3}$

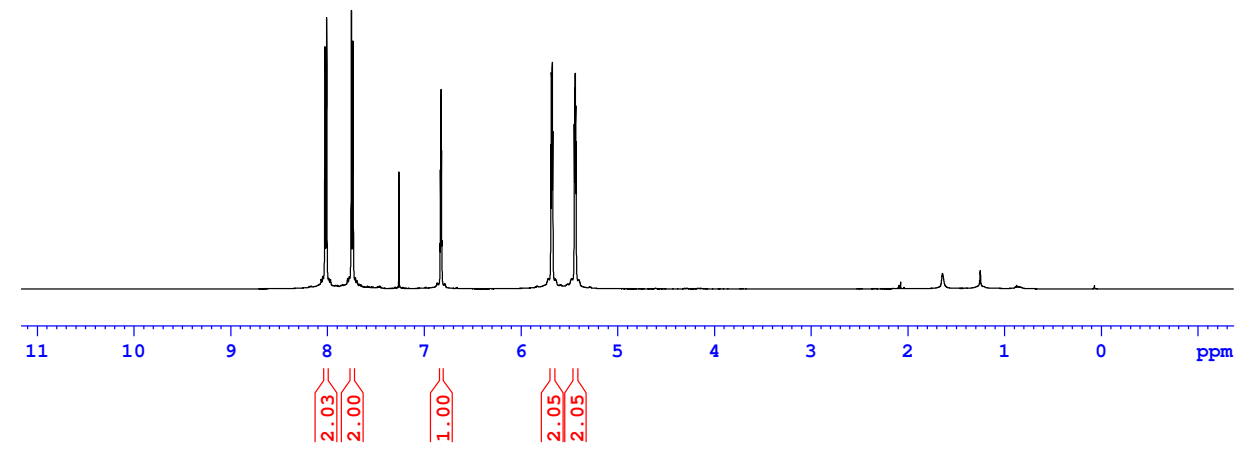

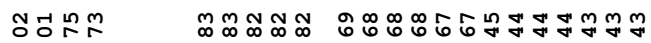

Dóvi

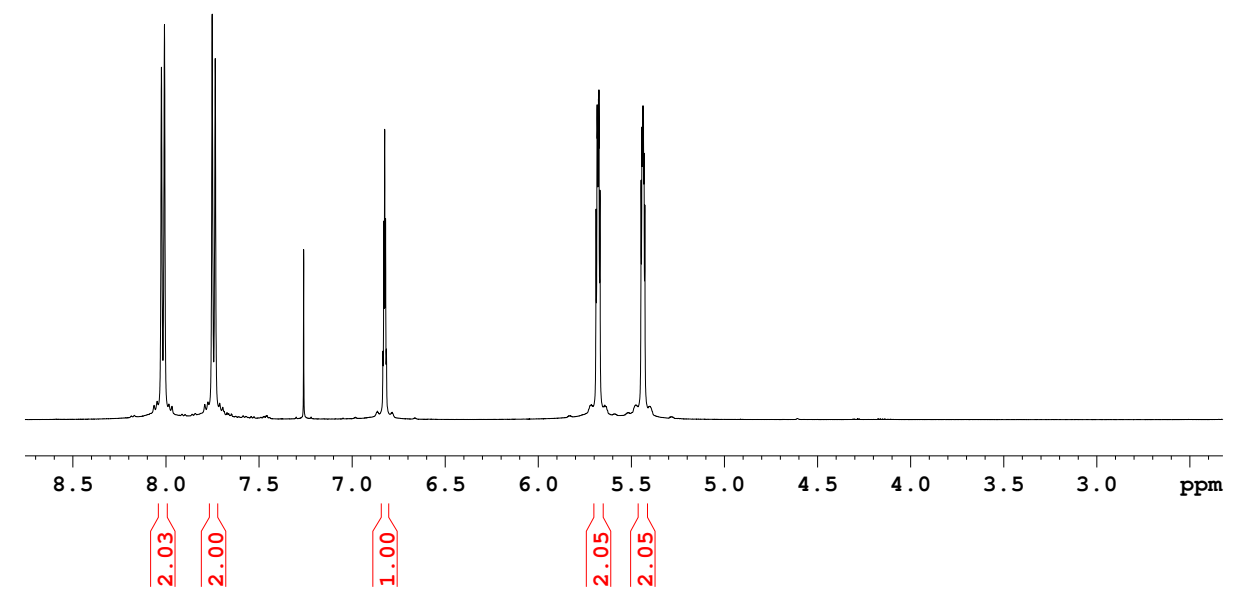



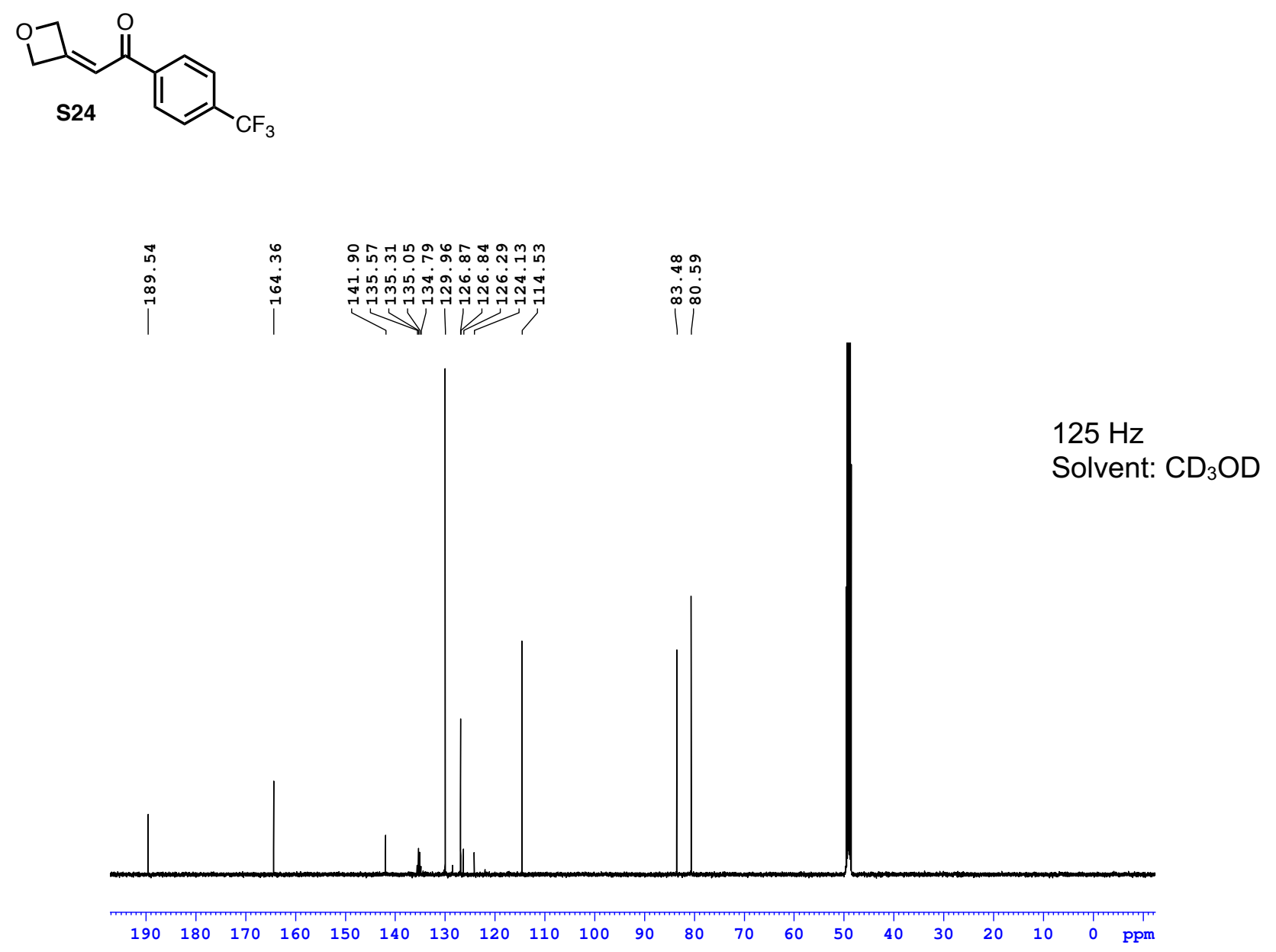
<smiles>O=Cc1occc1Cc1ccccc1</smiles>

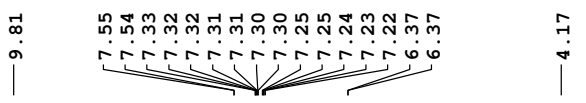

$500 \mathrm{~Hz}$

Solvent: $\mathrm{CDCl}_{3}$
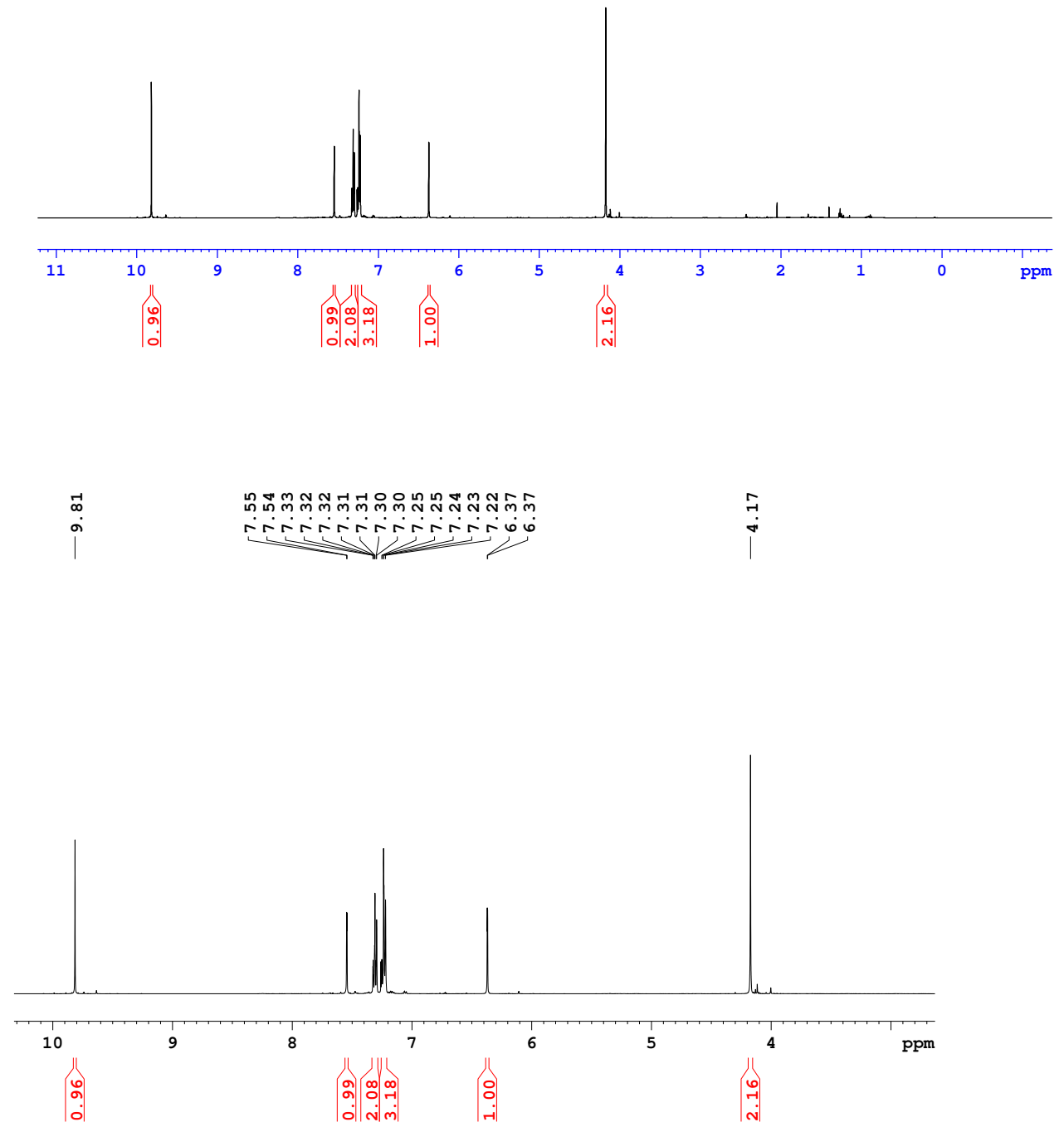
<smiles>O=Cc1occc1Cc1ccccc1</smiles>

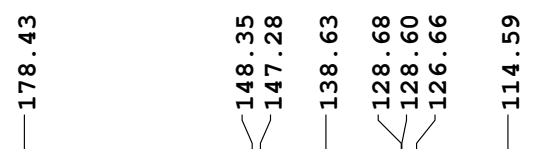

$\infty$

m

$125 \mathrm{~Hz}$

Solvent: $\mathrm{CDCl}_{3}$

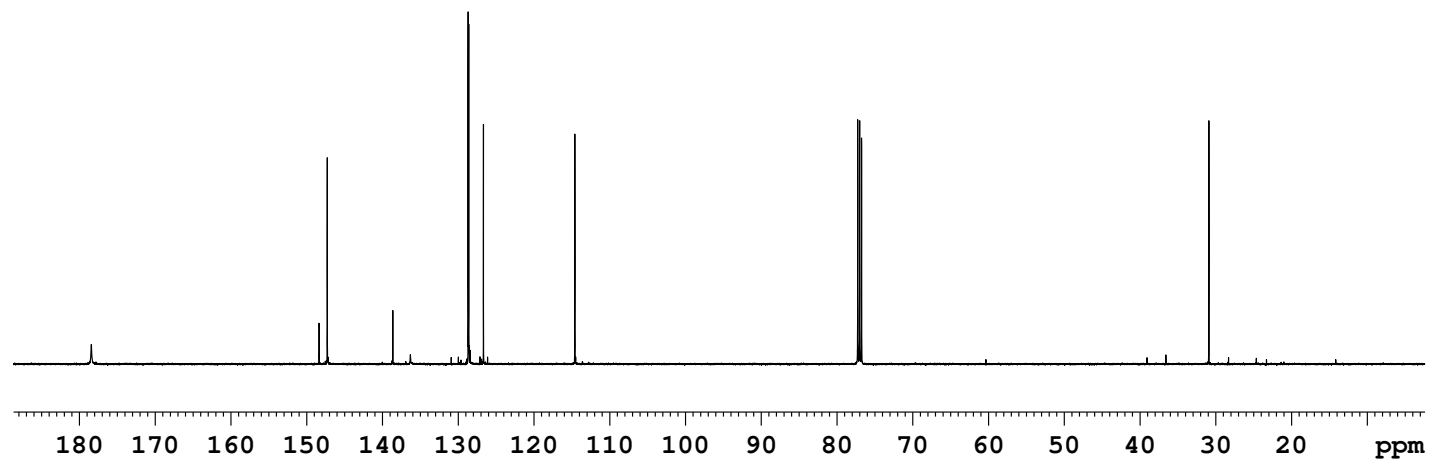


<smiles>OCc1occc1Cc1ccccc1</smiles>
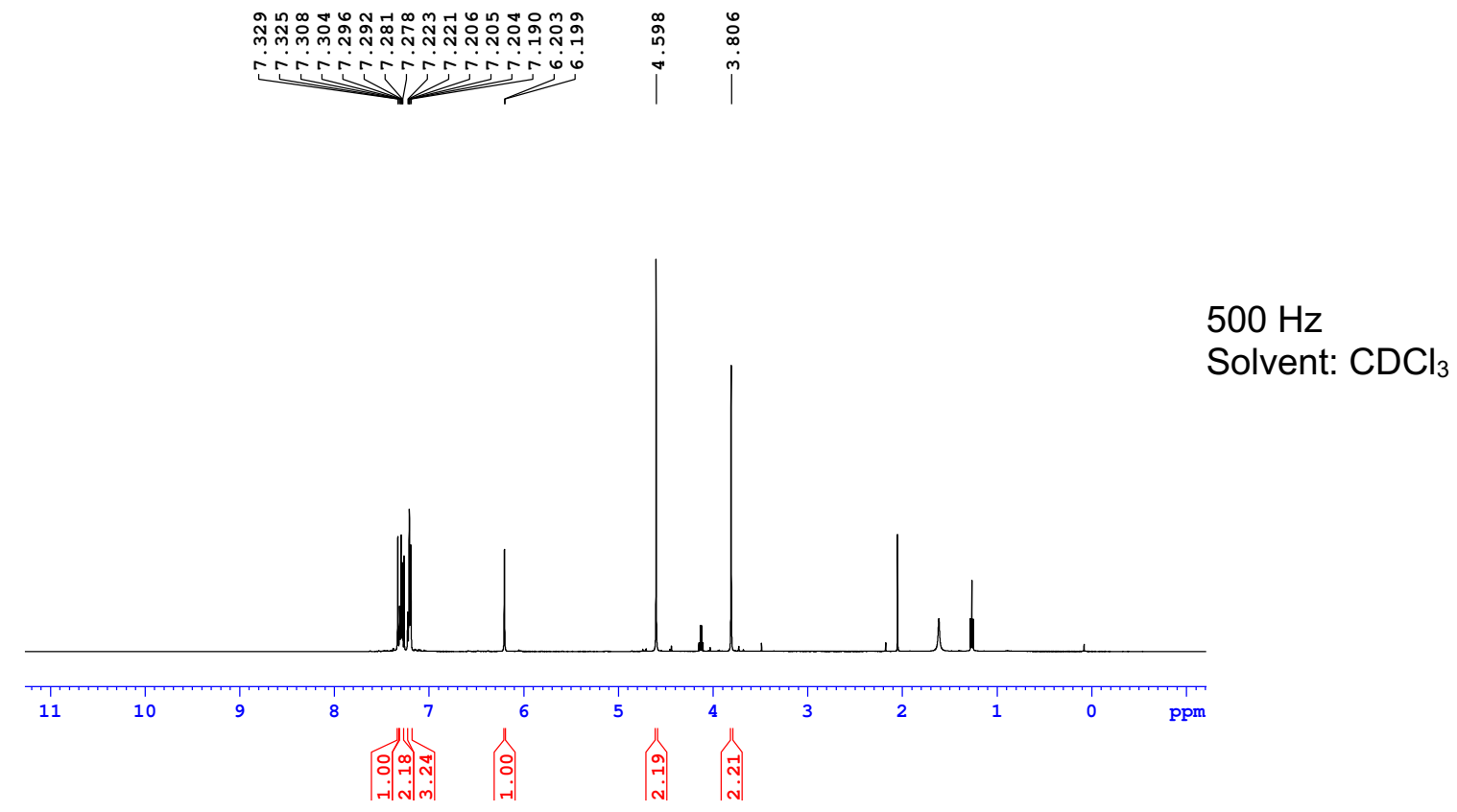

furan-2-oh-3-bz

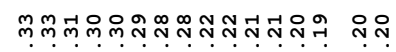

rininivinitr

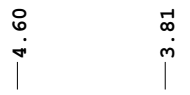

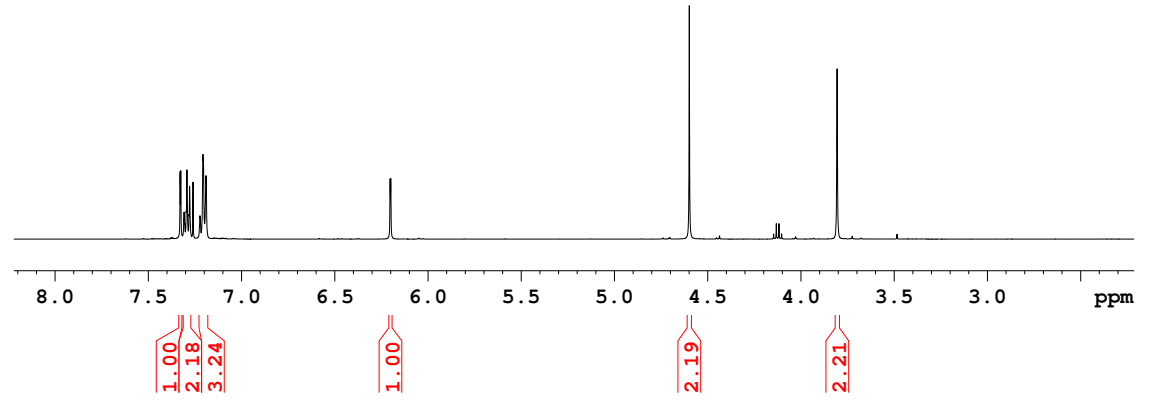


<smiles>OCc1occc1Cc1ccccc1</smiles>
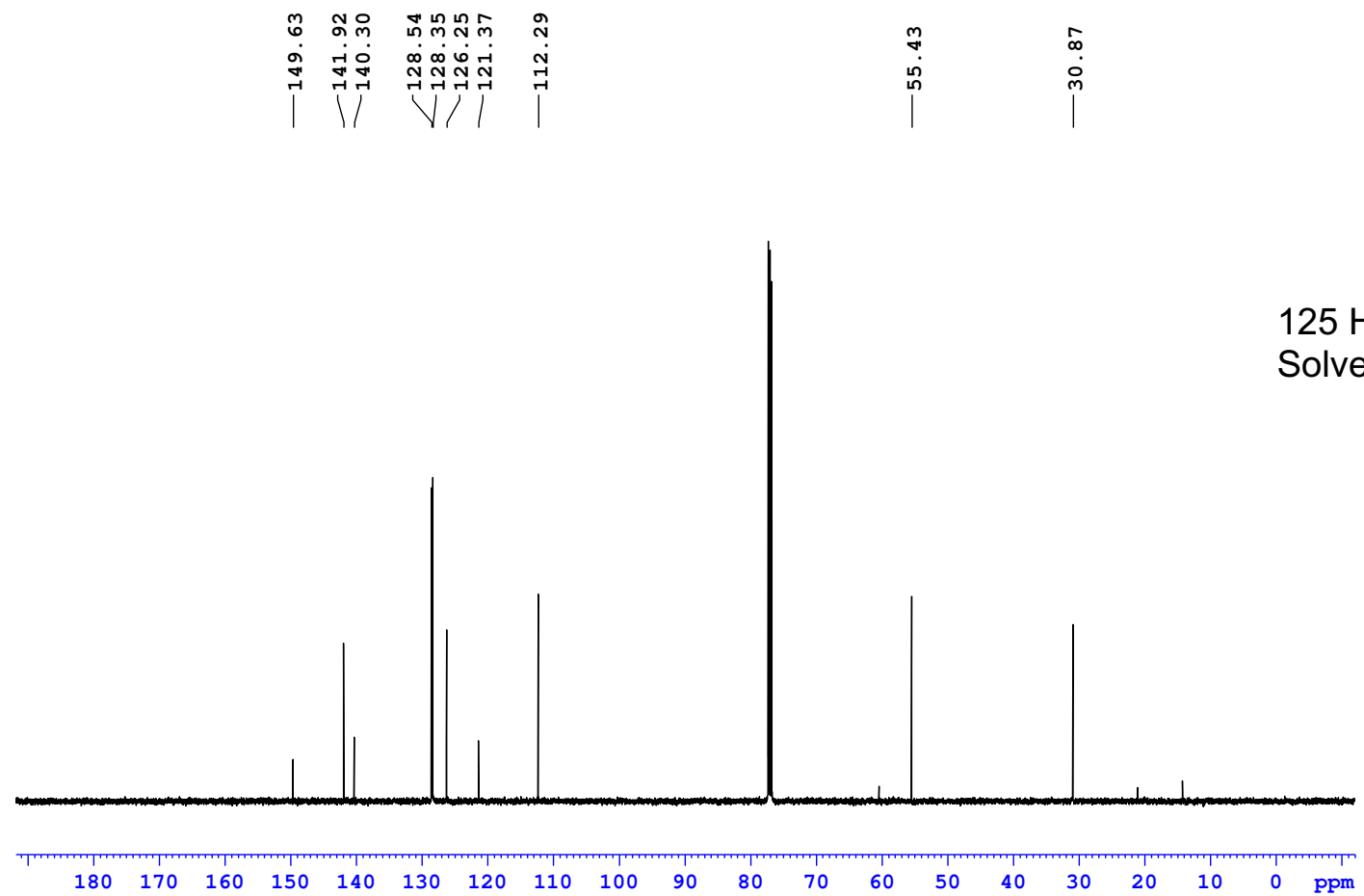

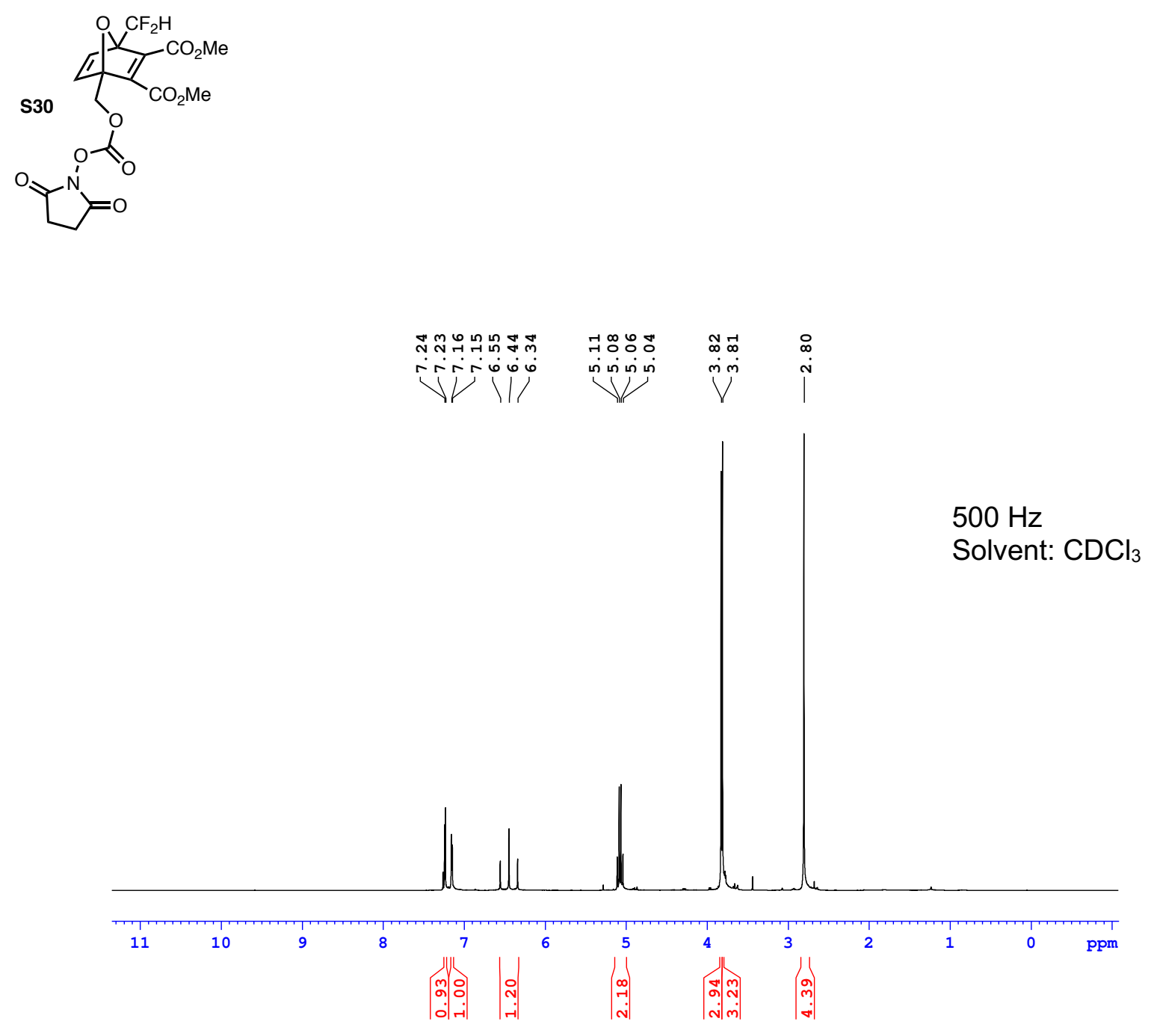


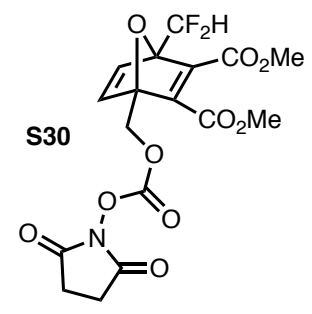

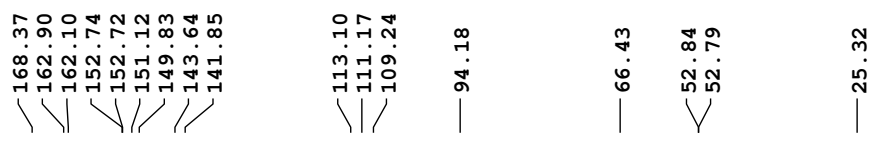

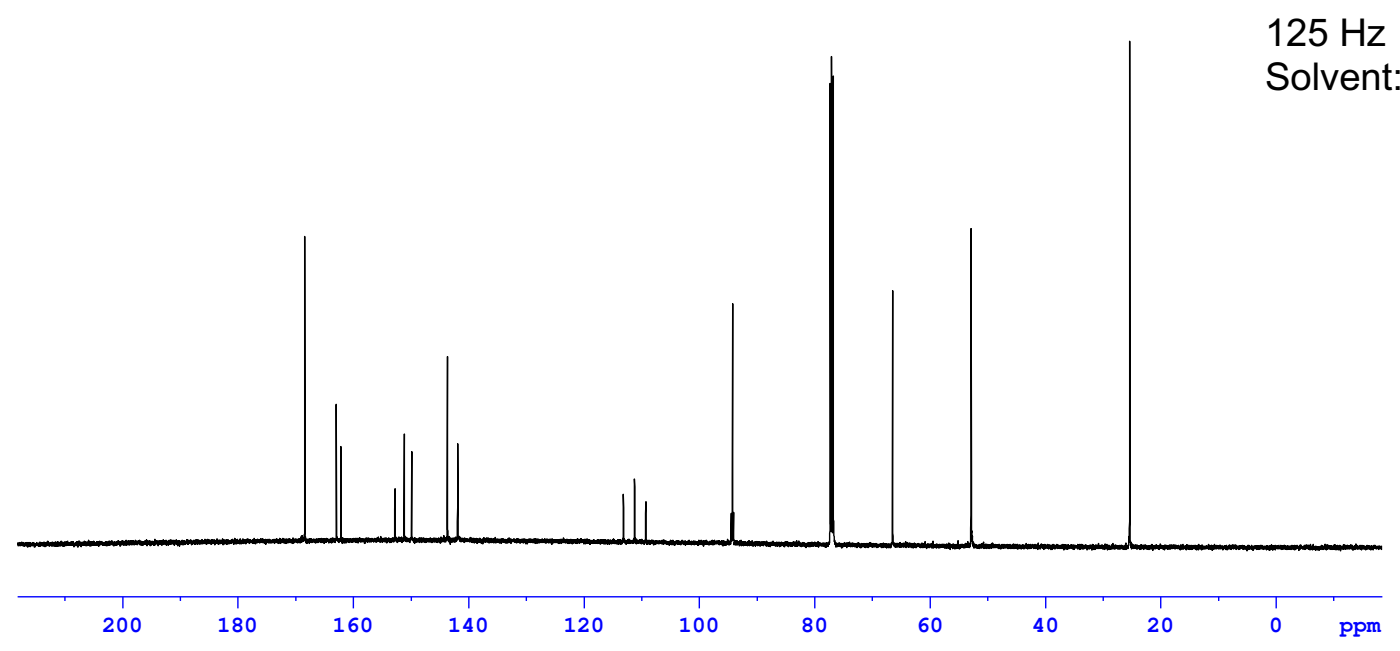



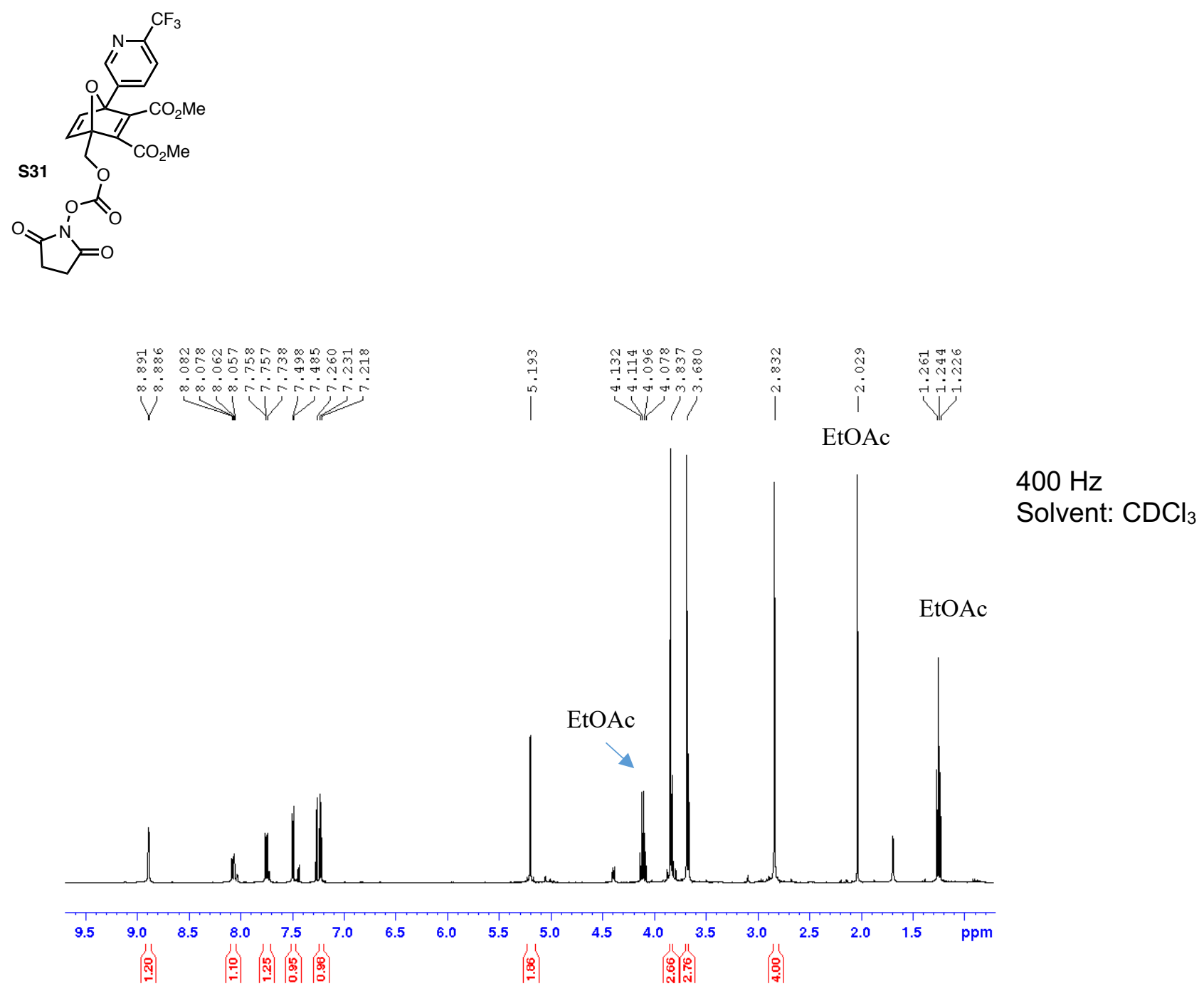

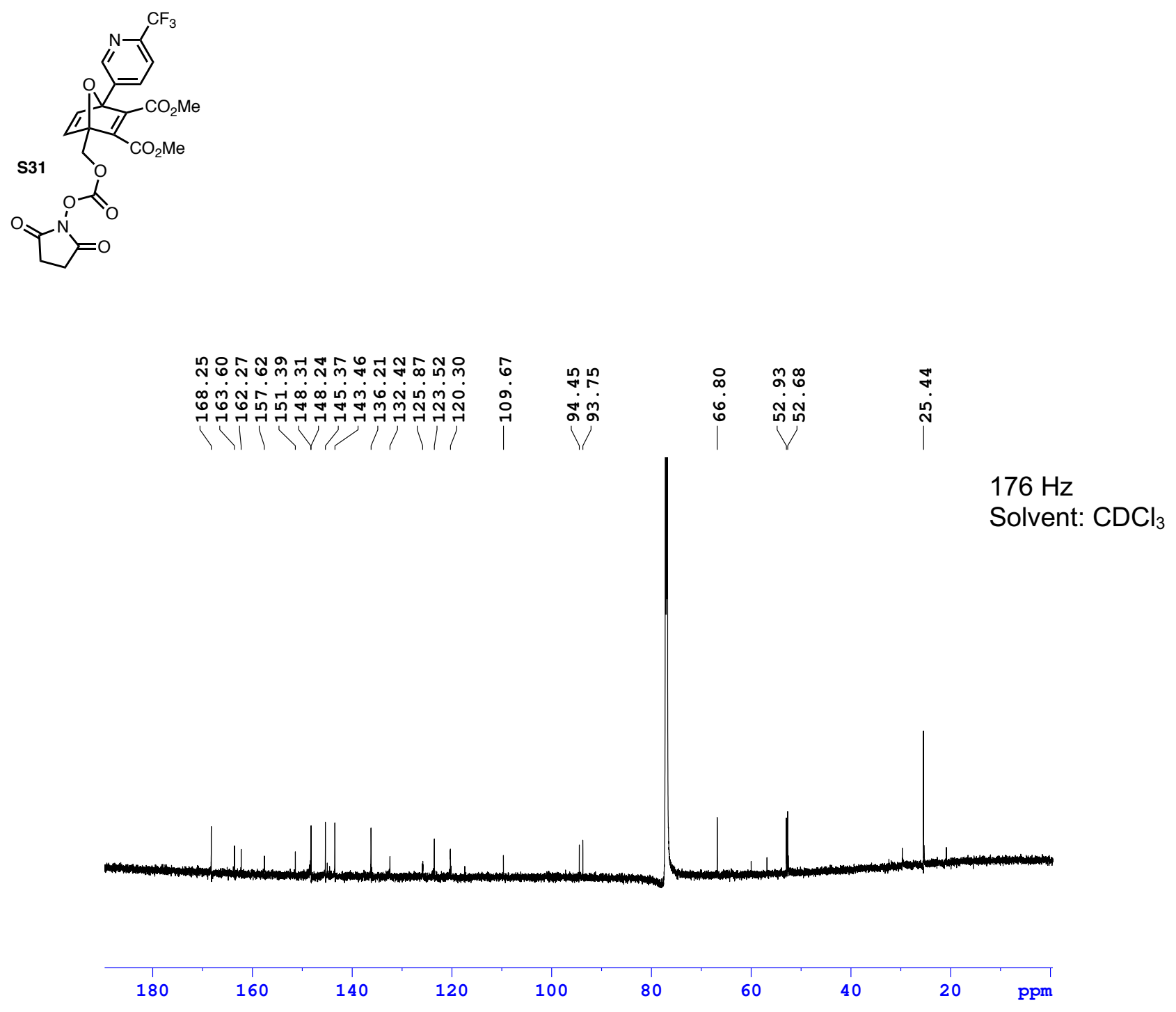

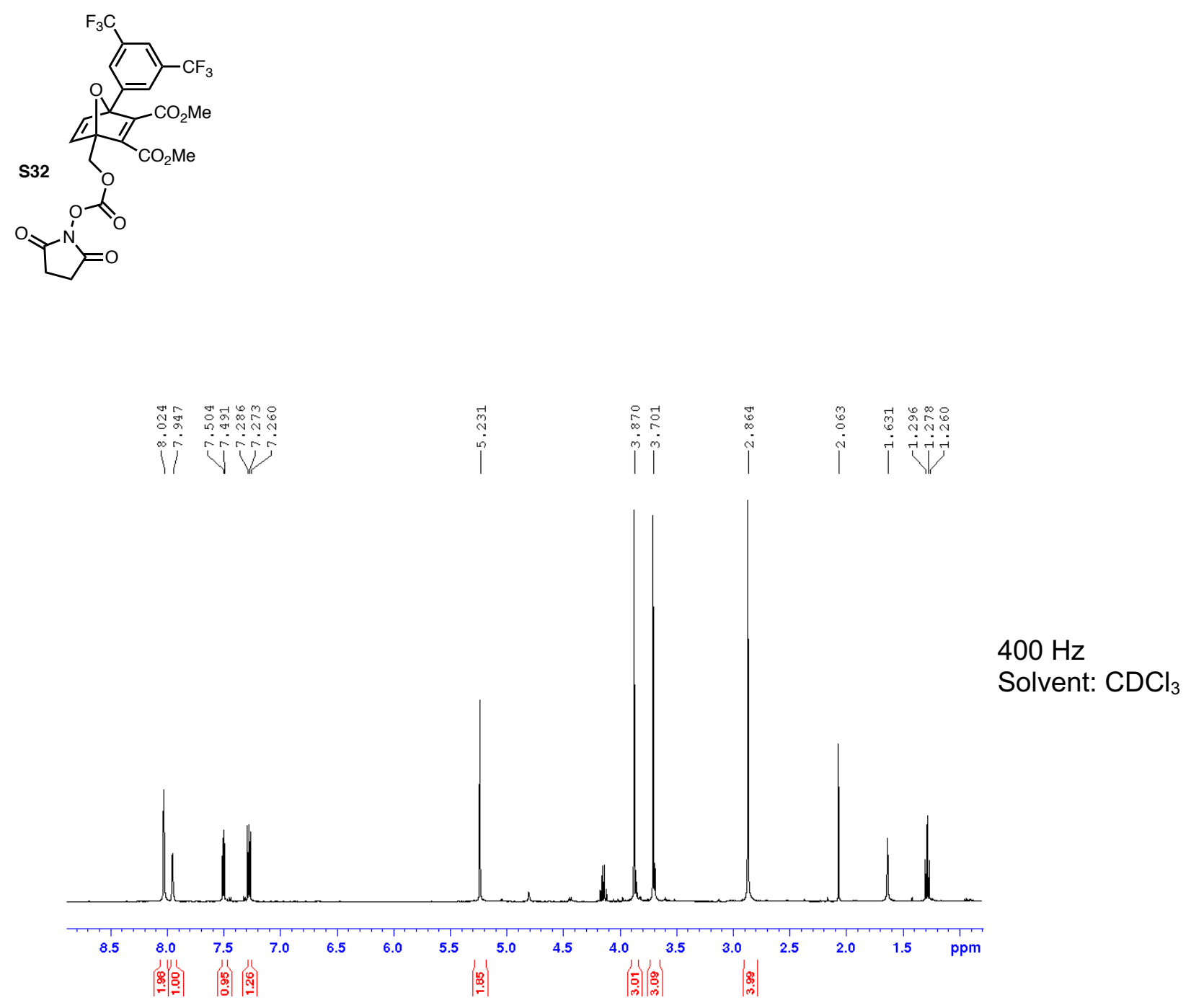


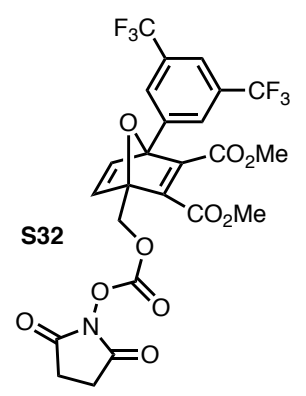

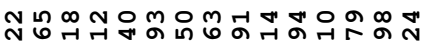

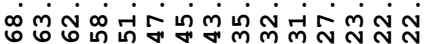

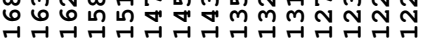
1)

กำ

ผू ตั

กू๊

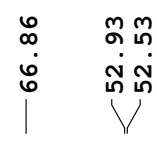

m

กุ่

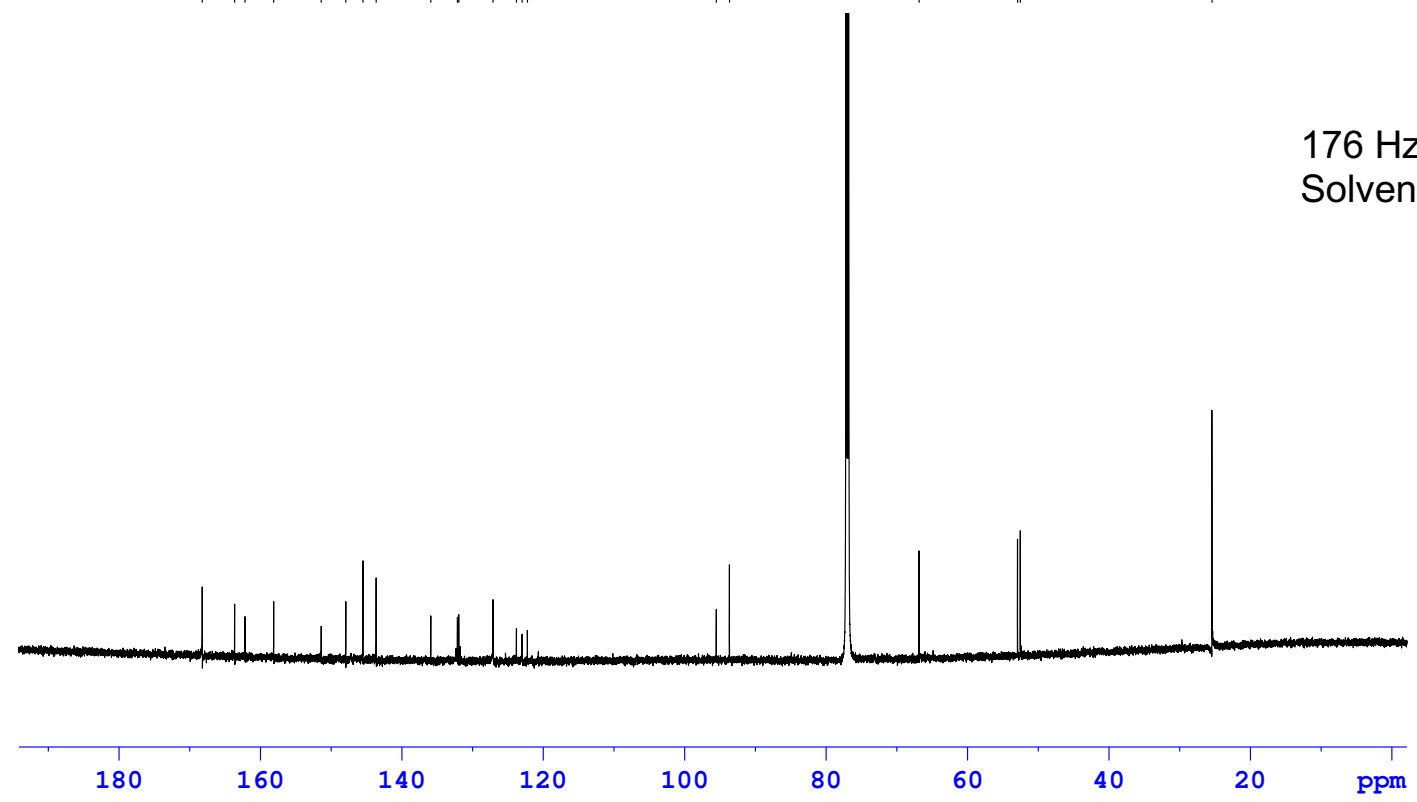




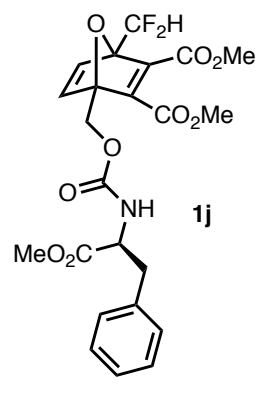

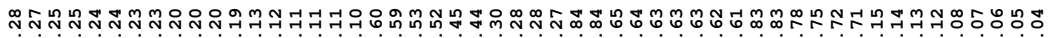

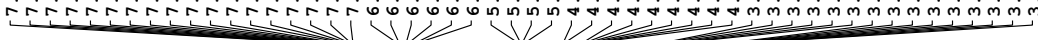

$700 \mathrm{~Hz}$

Solvent: $\mathrm{CDCl}_{3}$
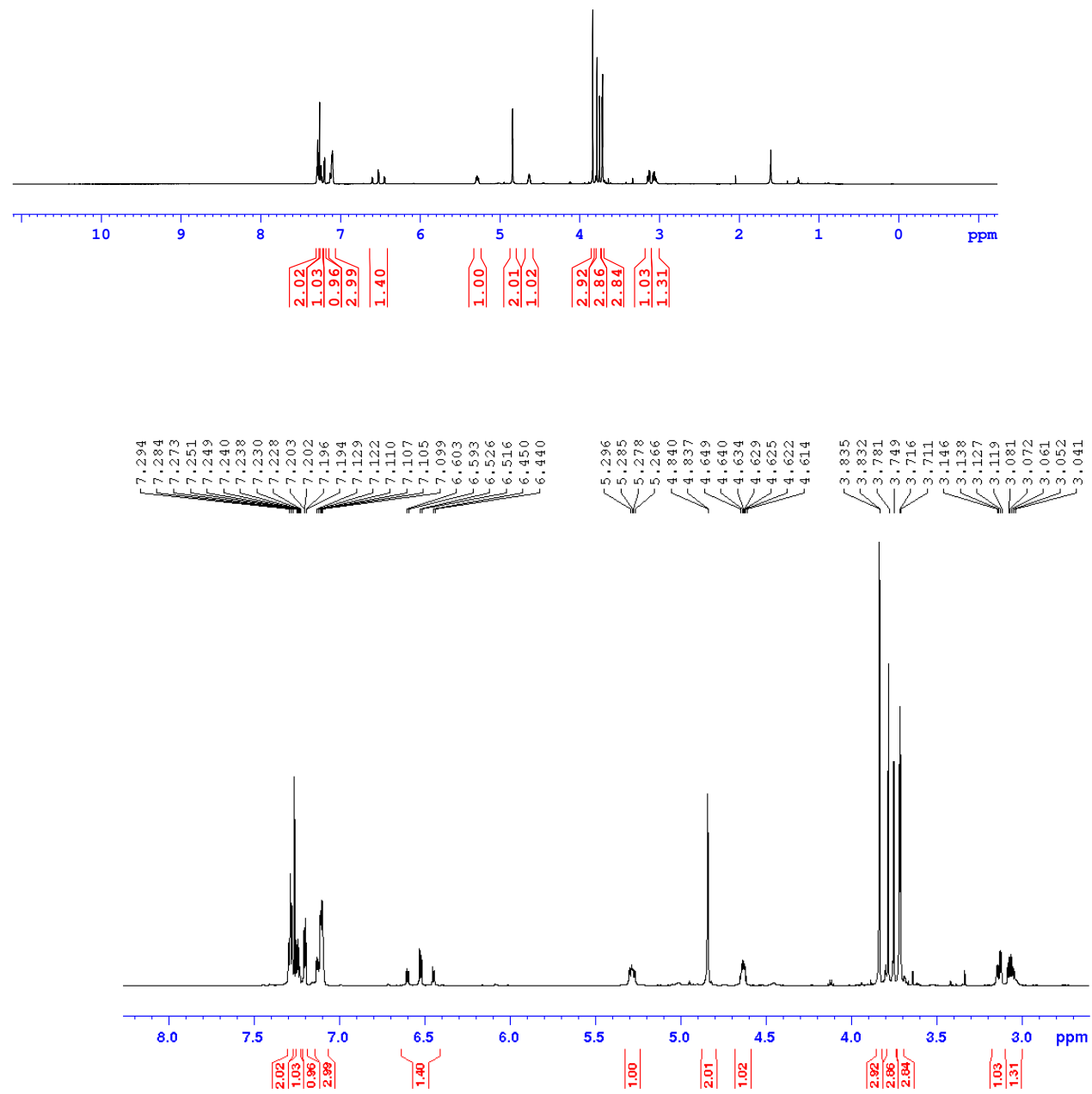


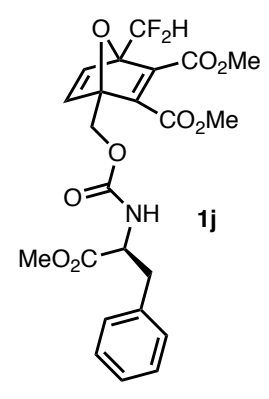

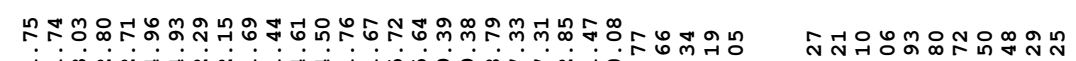

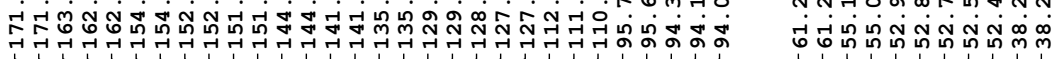

(N)

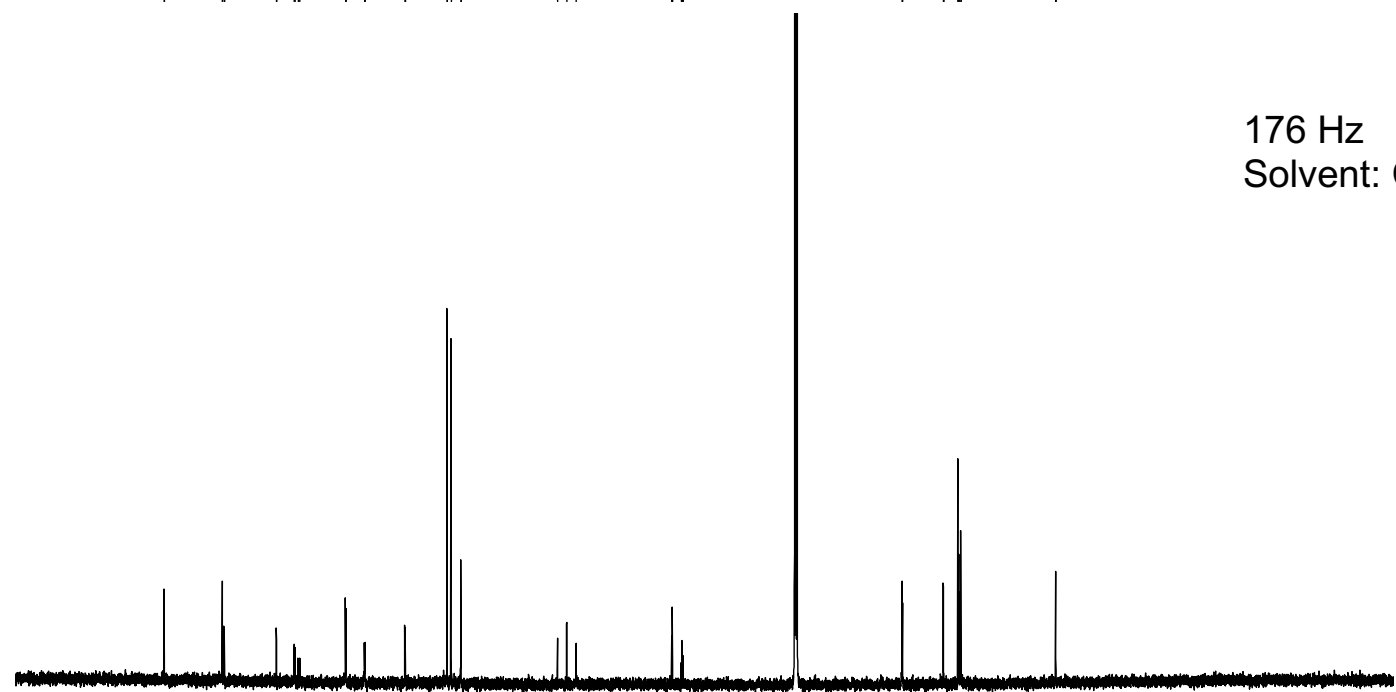

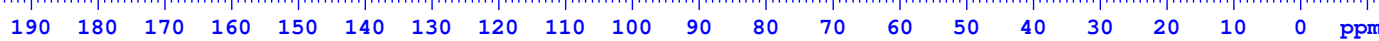




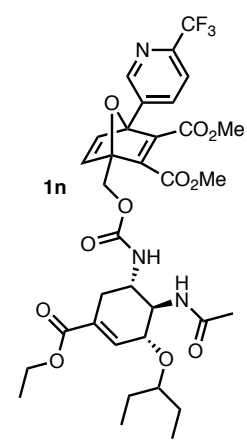

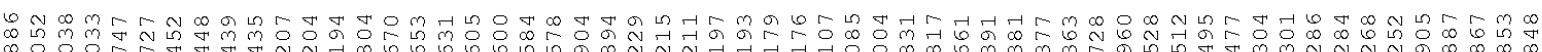

. . . . . . . . . .

I

$400 \mathrm{~Hz}$

Solvent: $\mathrm{CDCl}_{3}$
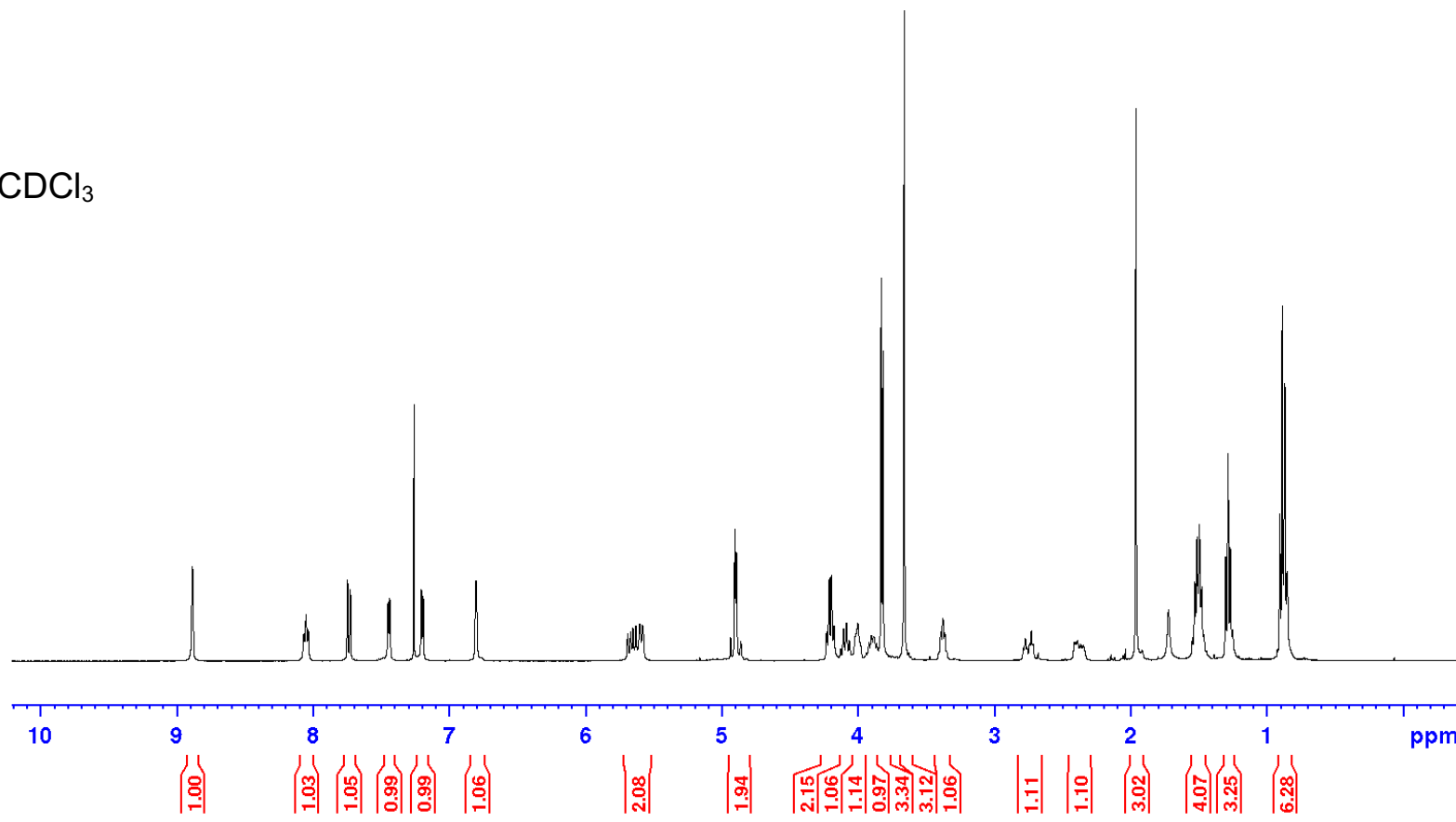


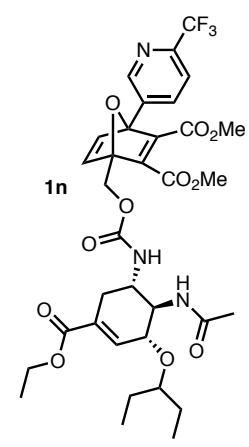

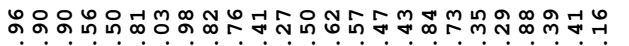

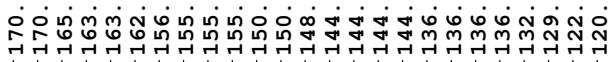

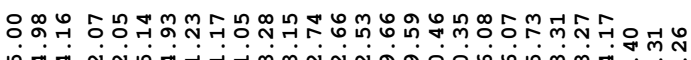

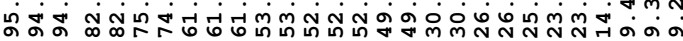
$\longrightarrow v /$

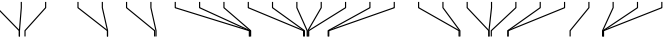

$125 \mathrm{~Hz}$

Solvent: $\mathrm{CDCl}_{3}$
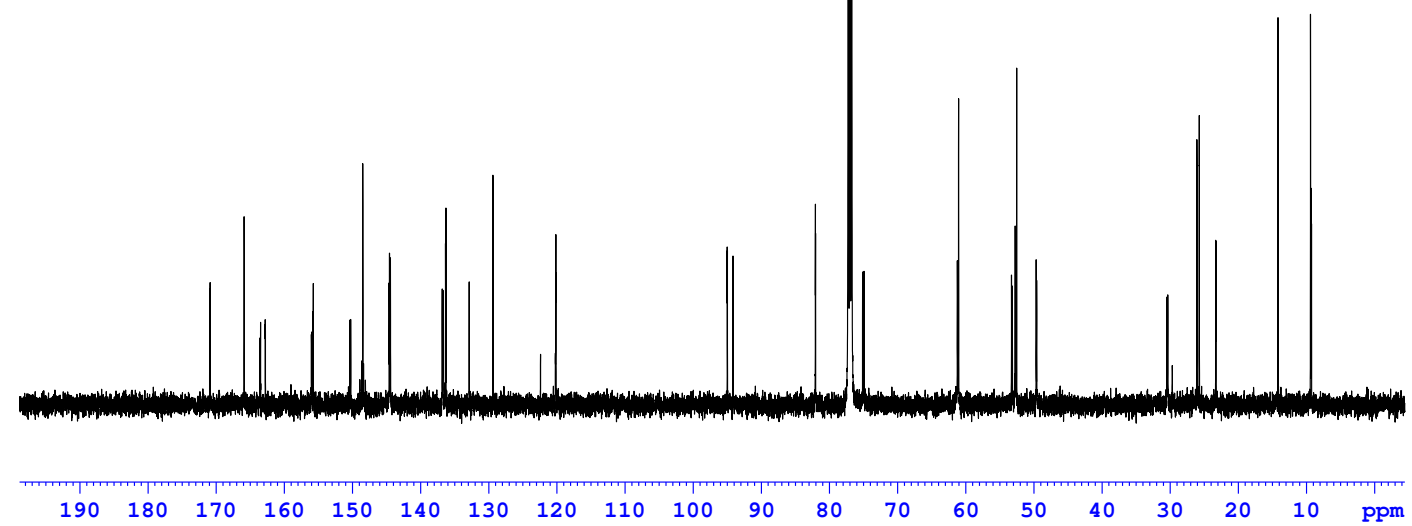

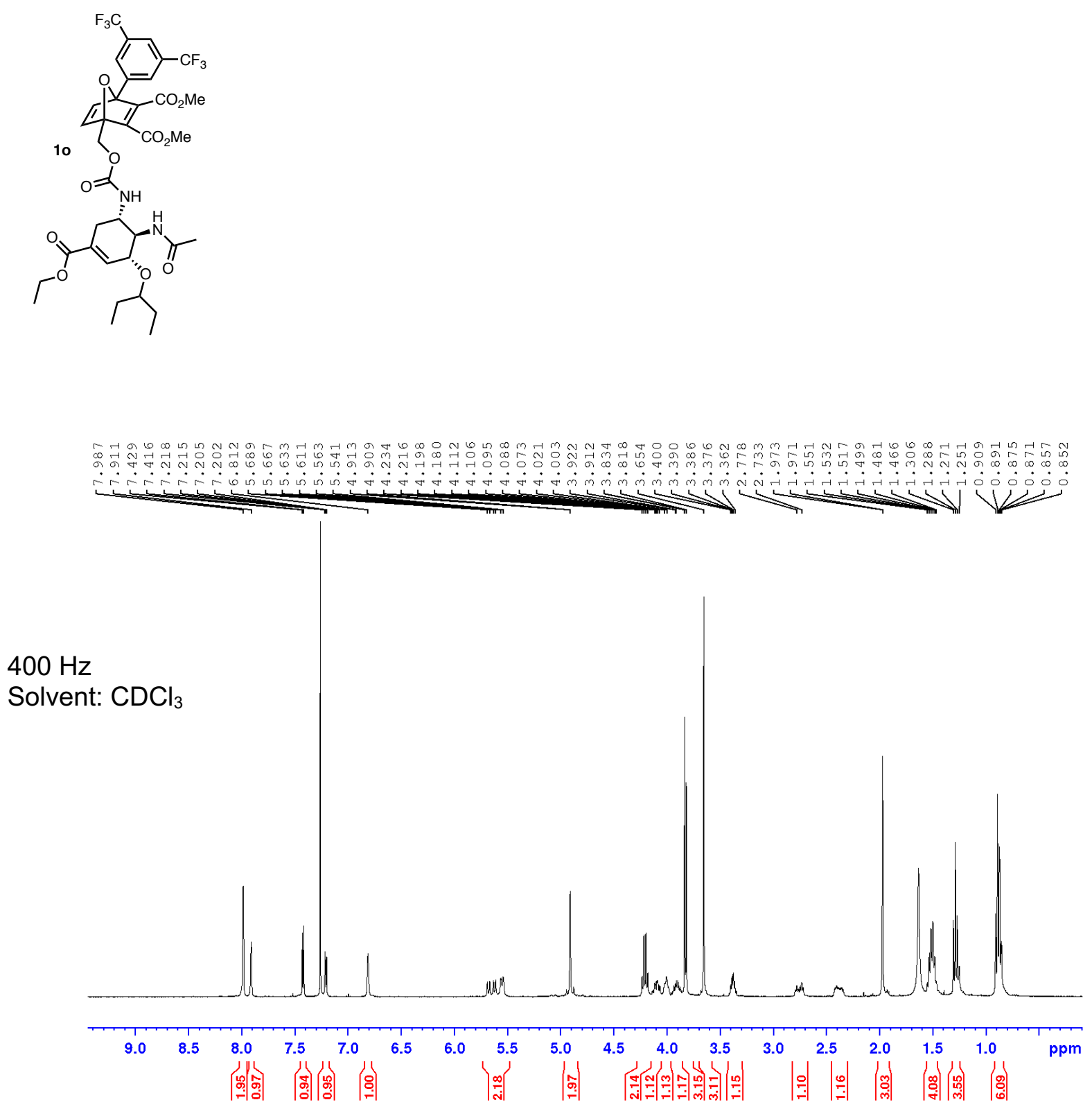


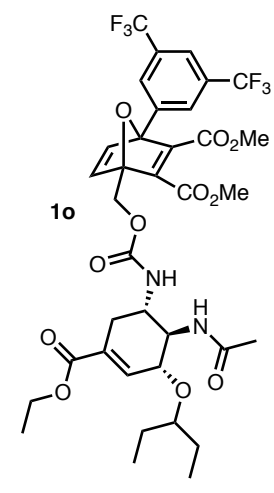

ูํㅇำ

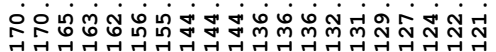

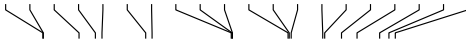

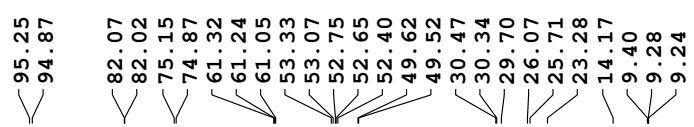

$176 \mathrm{~Hz}$

Solvent: $\mathrm{CDCl}_{3}$

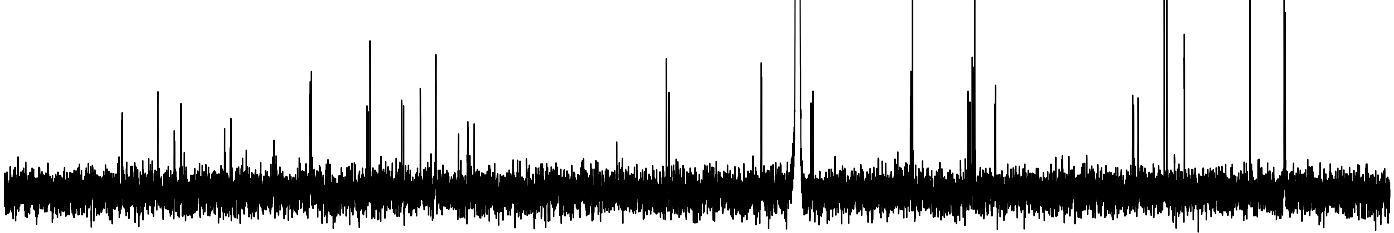

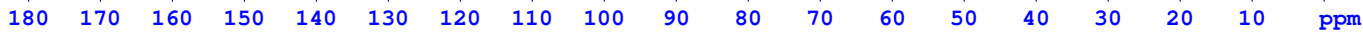




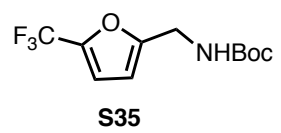

עं।

$500 \mathrm{~Hz}$

Solvent: $\mathrm{CDCl}_{3}$

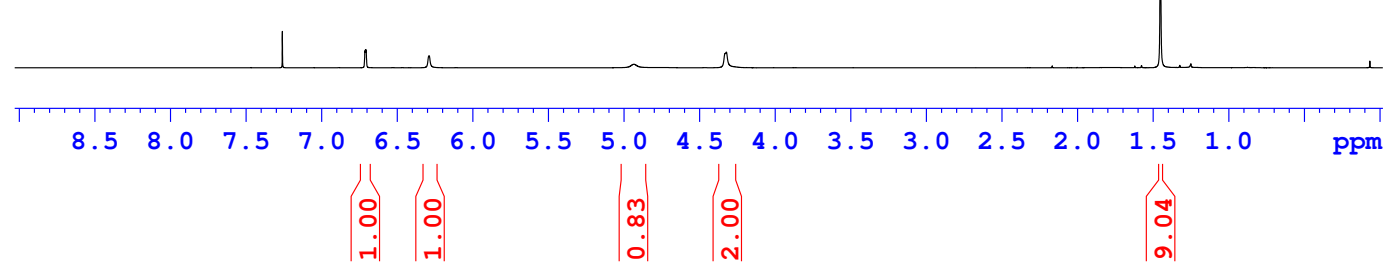



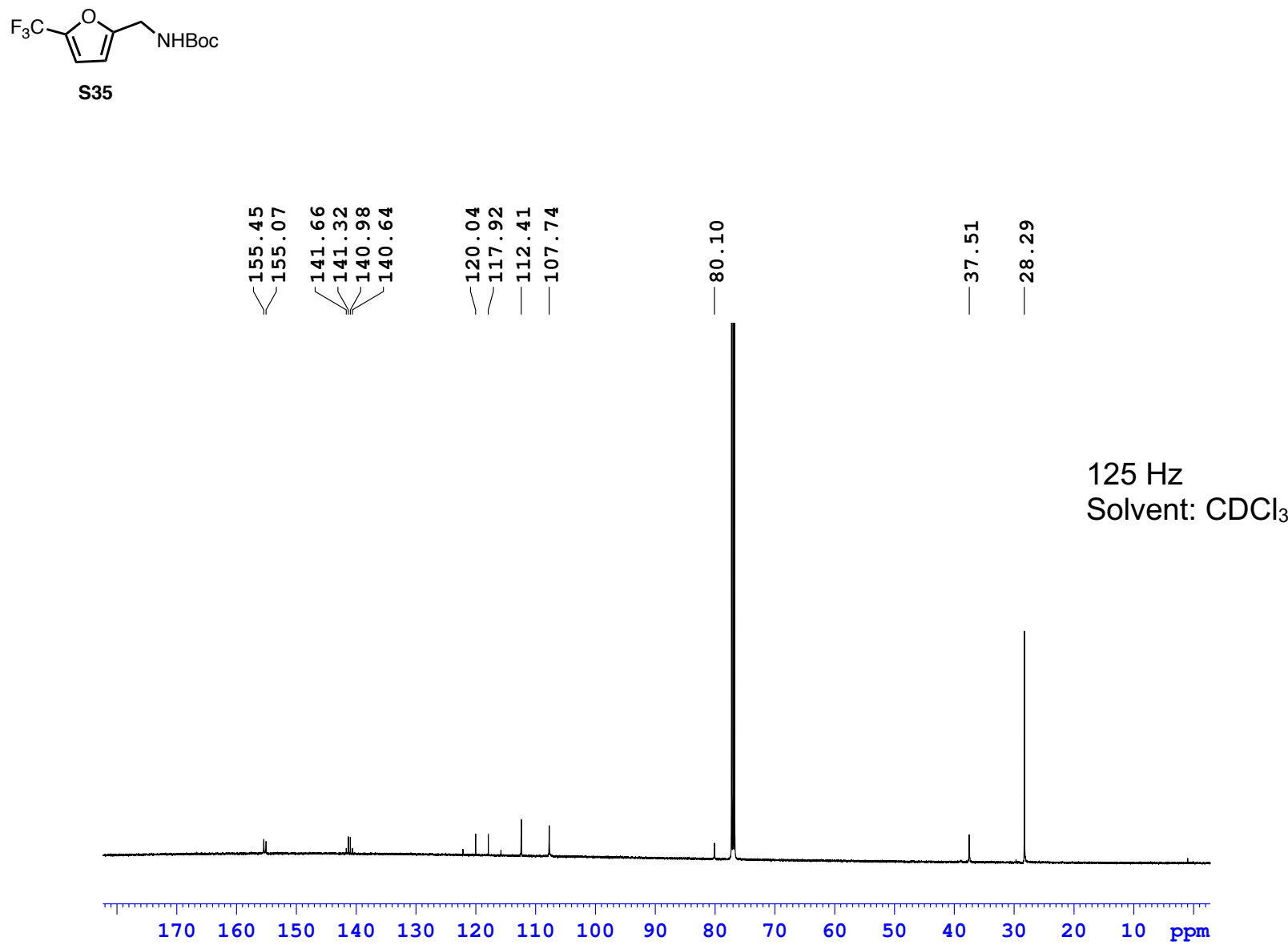

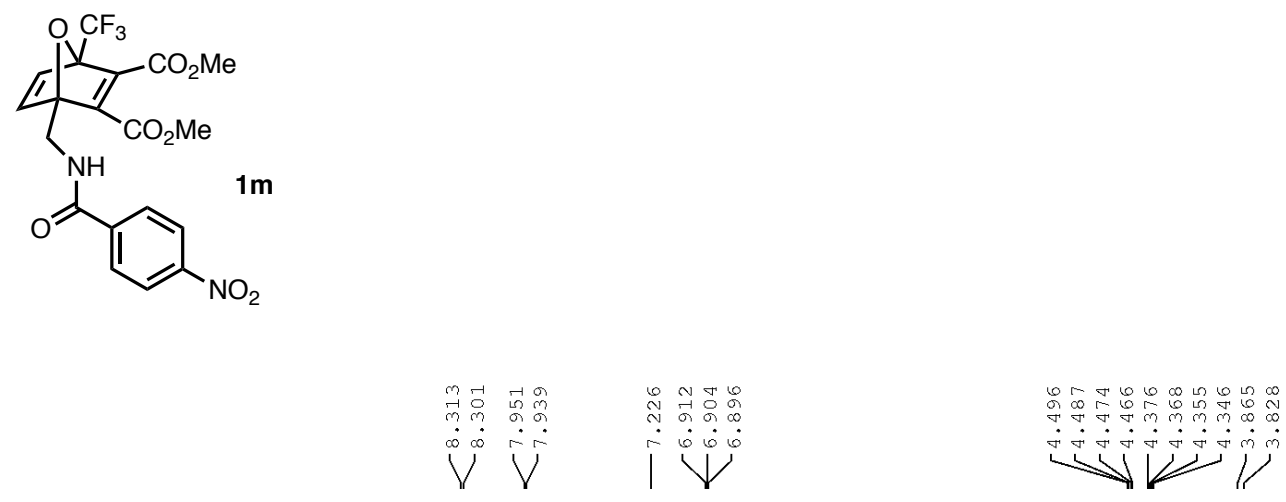

$700 \mathrm{~Hz}$

Solvent: $\mathrm{CDCl}_{3}$

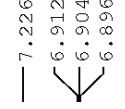

$+1$

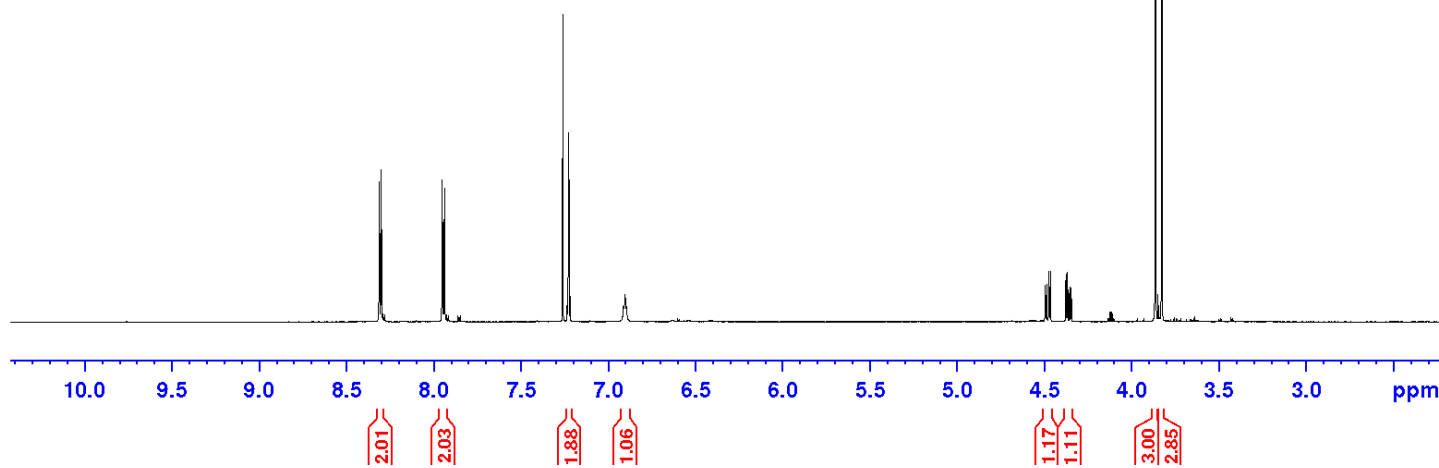



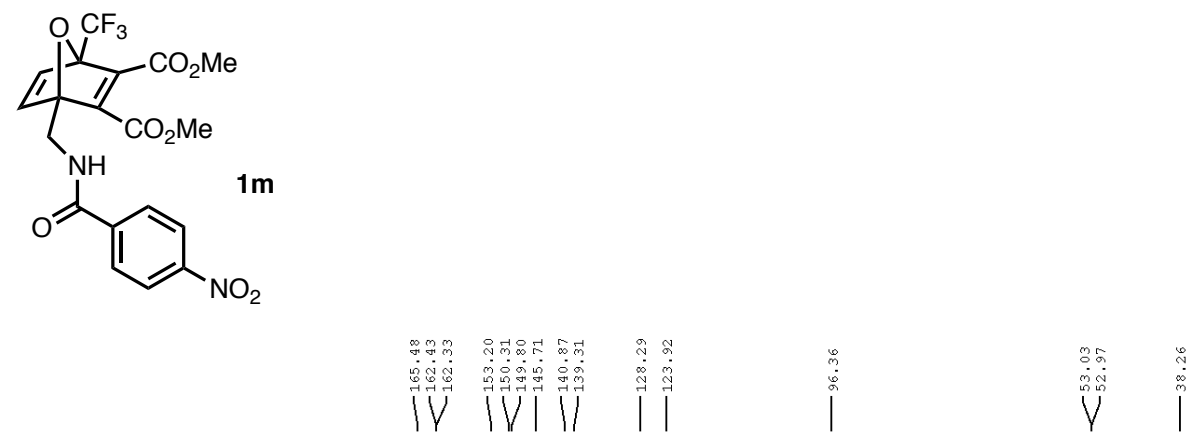

$176 \mathrm{~Hz}$

Solvent: $\mathrm{CDCl}_{3}$

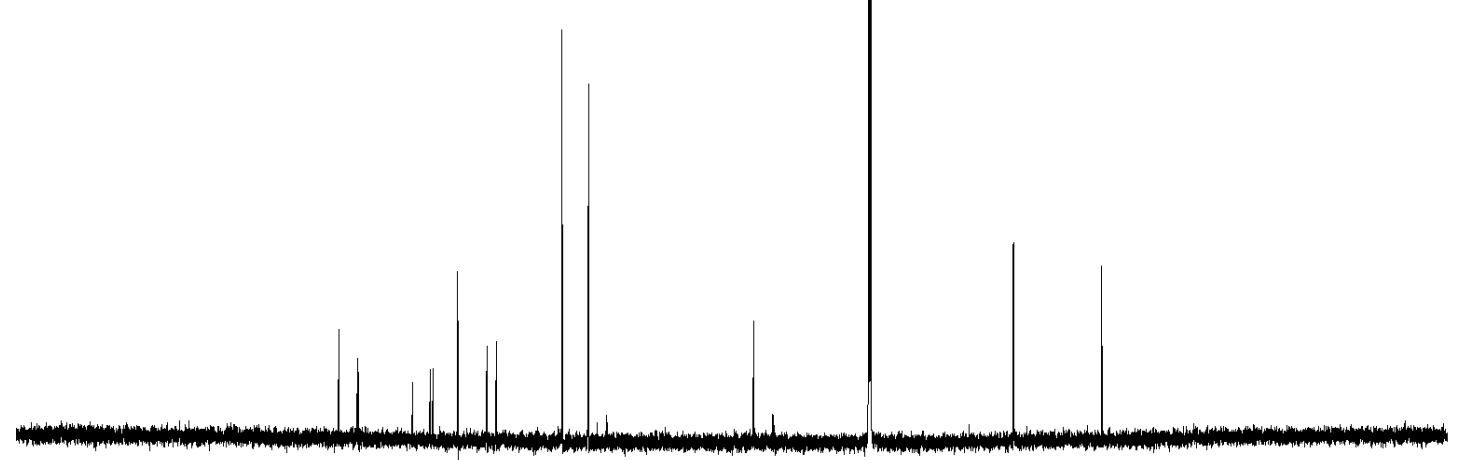

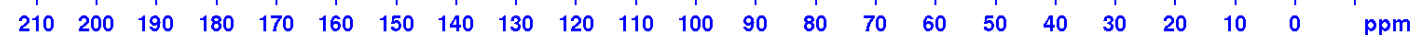


<smiles>O=Cc1ccc(-c2cc(C(F)(F)F)cc(C(F)(F)F)c2)o1</smiles>

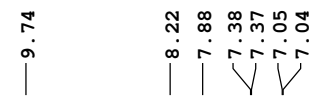

$400 \mathrm{~Hz}$

Solvent: $\mathrm{CDCl}_{3}$
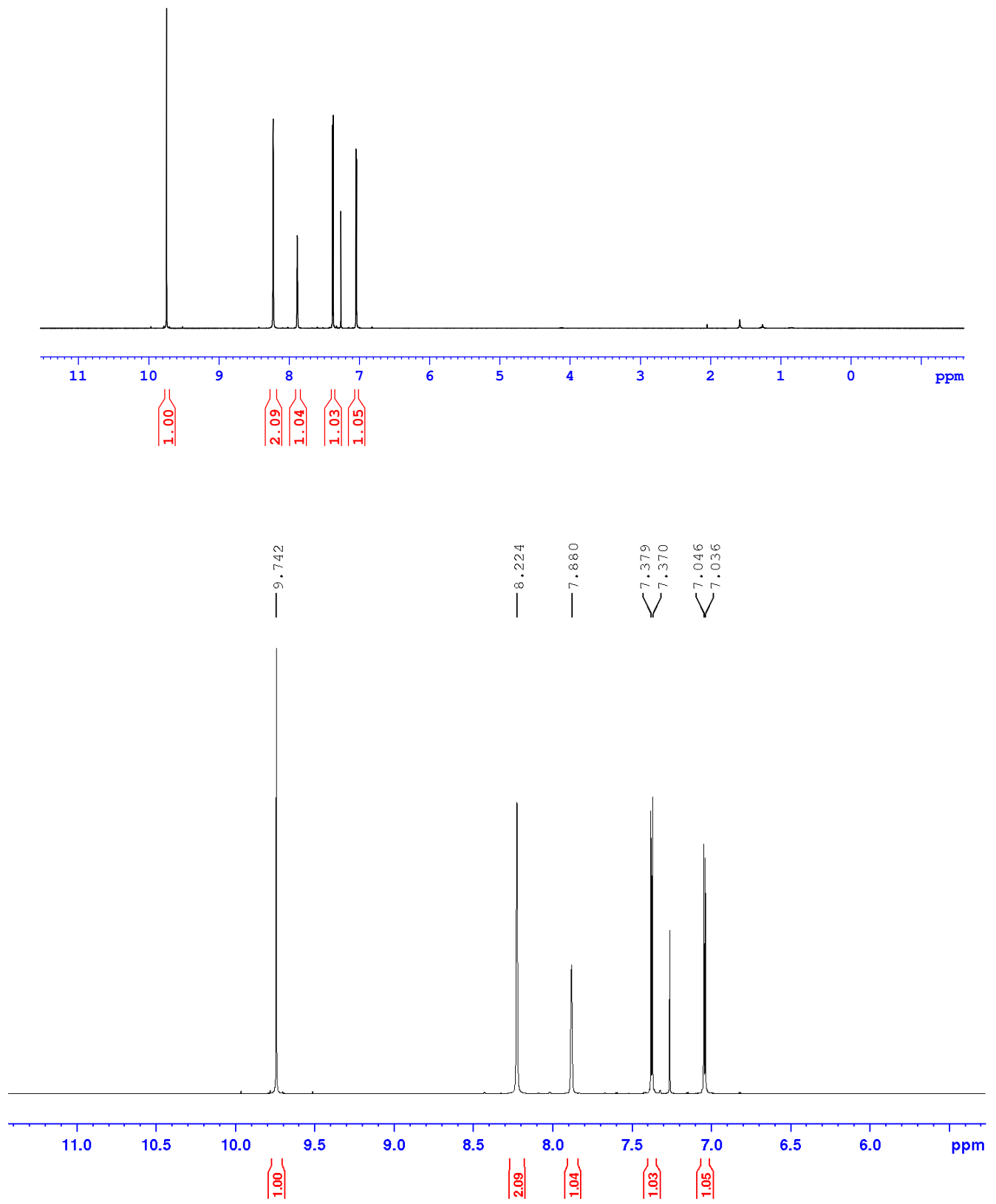
<smiles>O=Cc1ccc(-c2cc(C(F)(F)F)cc(C(F)(F)F)c2)o1</smiles>

$100 \mathrm{~Hz}$

Solvent: $\mathrm{CDCl}_{3}$

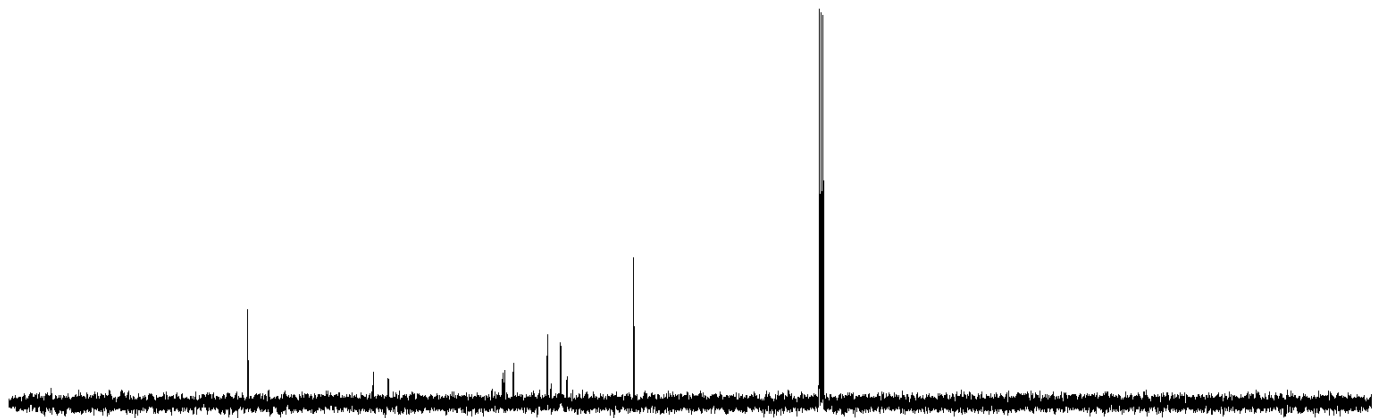

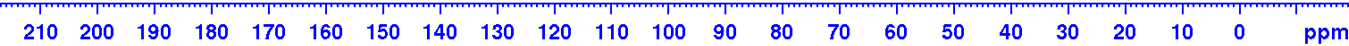




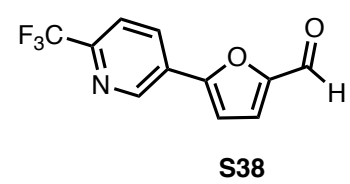

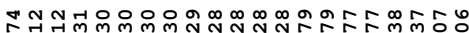

O人,

$400 \mathrm{~Hz}$

Solvent: $\mathrm{CDCl}_{3}$
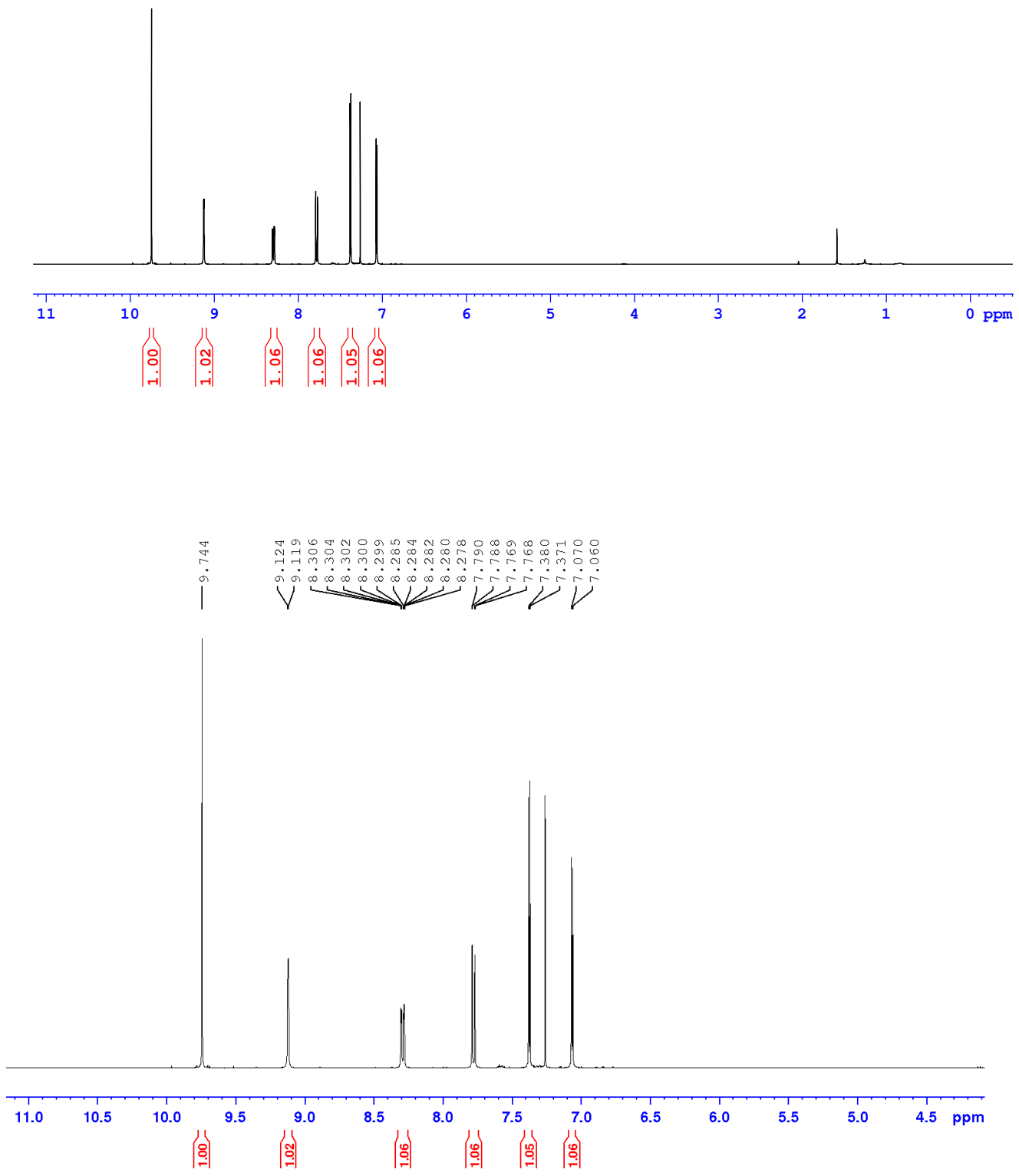

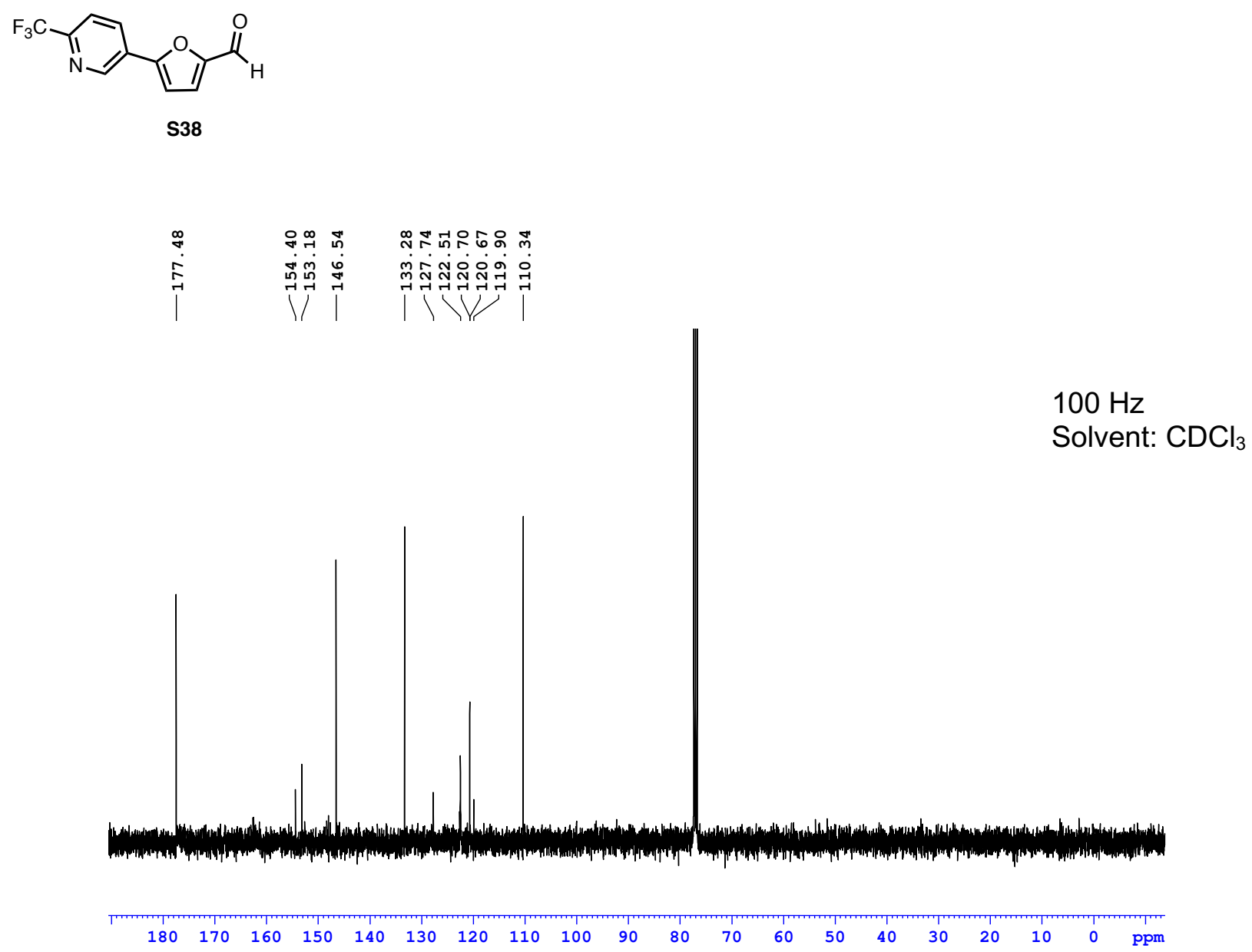
<smiles>OCc1ccc(-c2cc(C(F)(F)F)cc(C(F)(F)F)c2)o1</smiles>

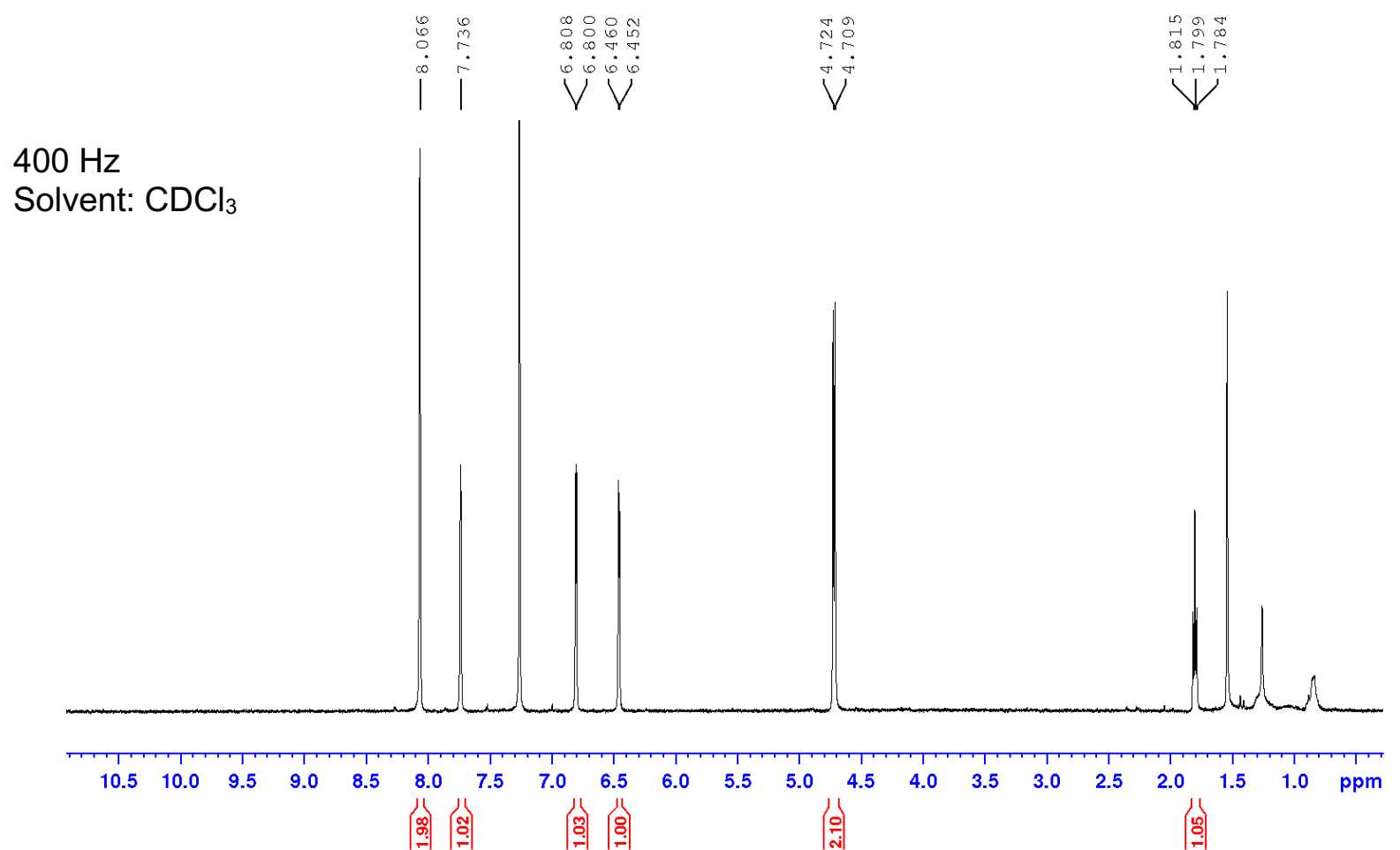


<smiles>OCc1ccc(-c2cc(C(F)(F)F)cc(C(F)(F)F)c2)o1</smiles>

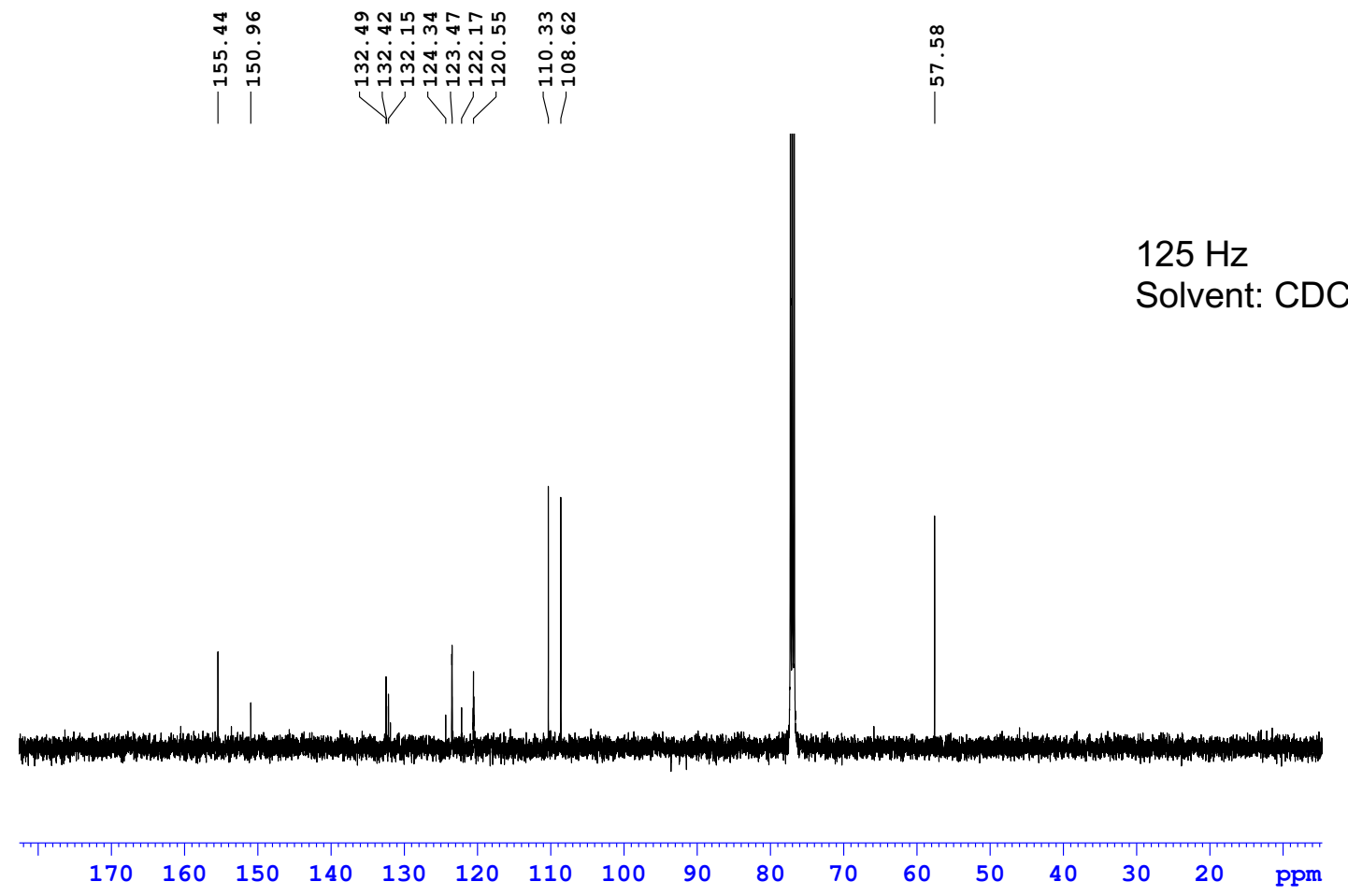




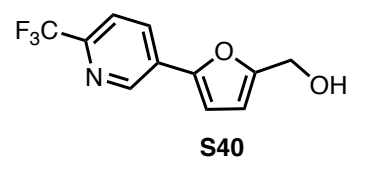

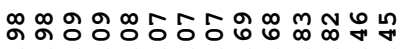

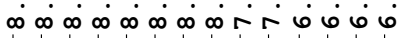

$V^{\infty} V^{\infty}$
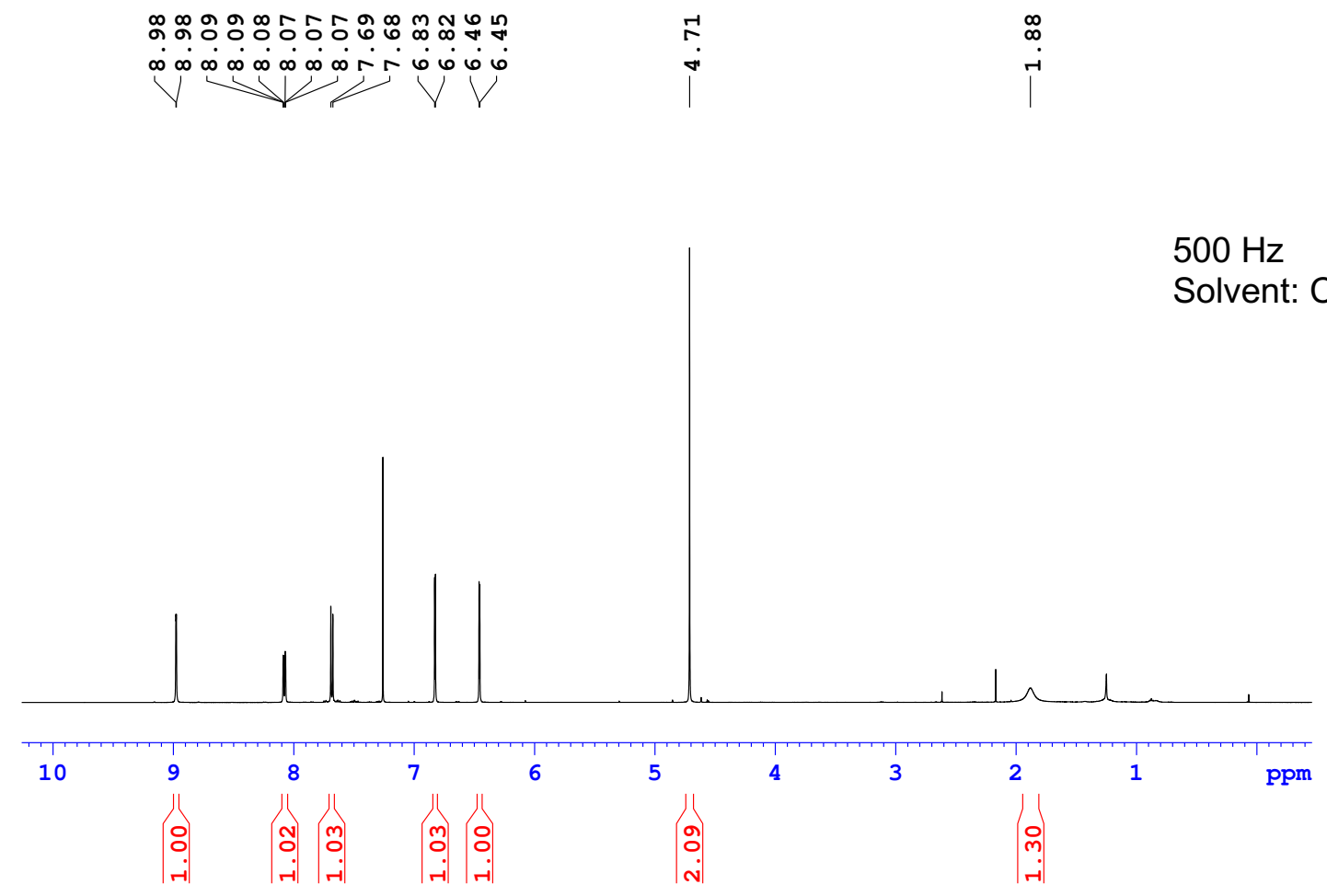

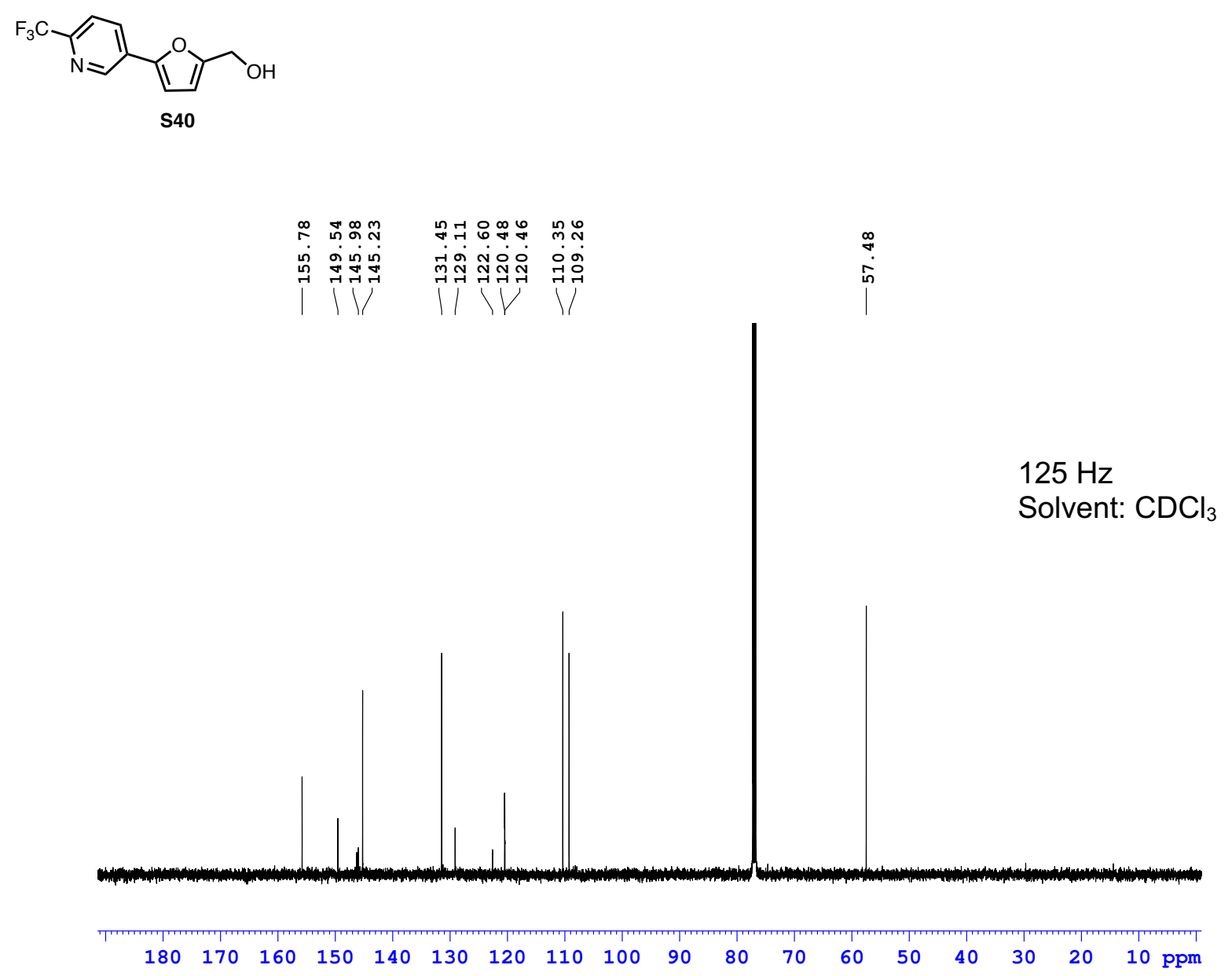

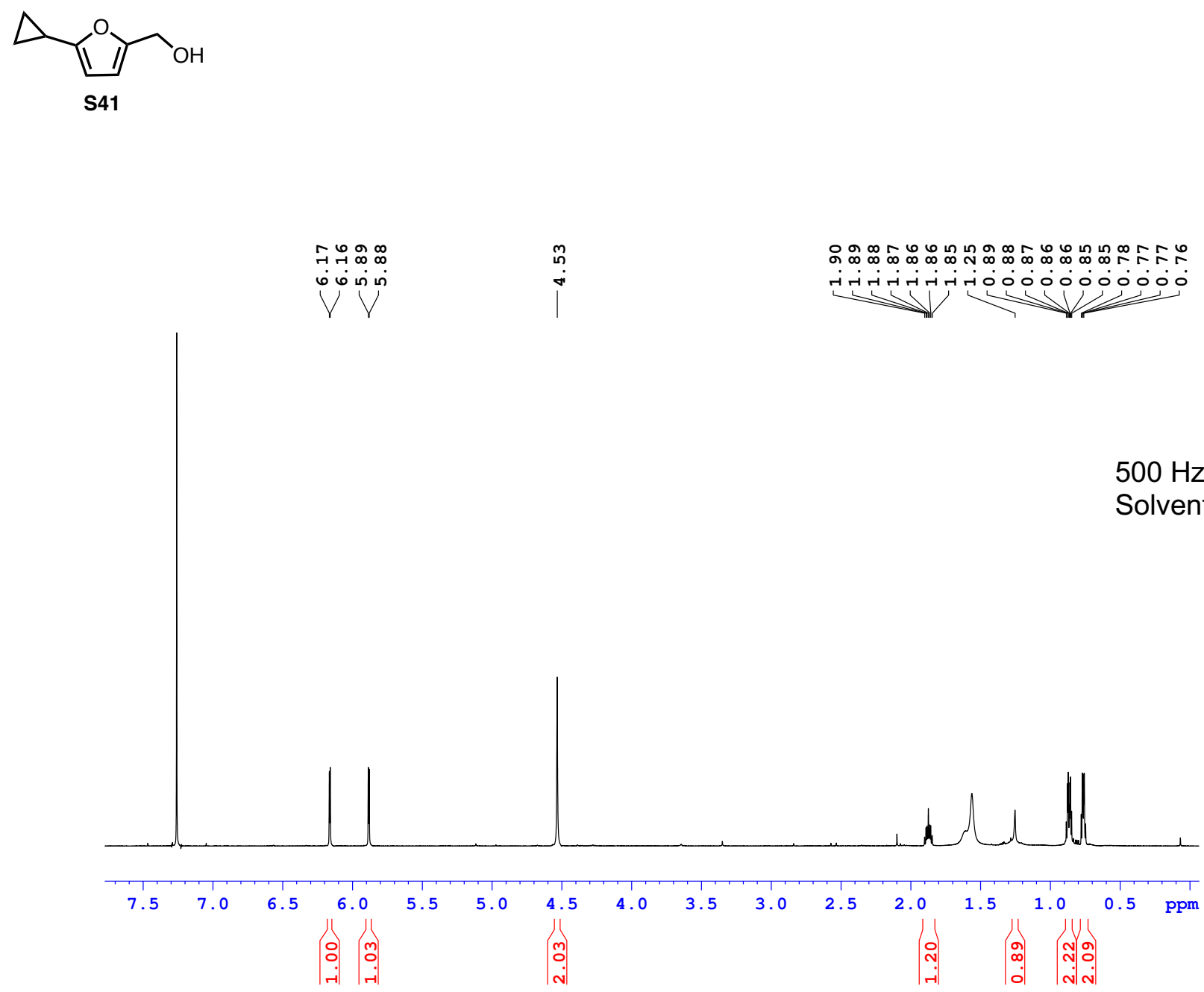


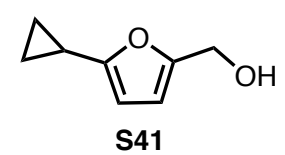

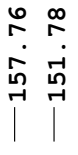

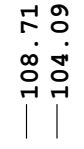

$\stackrel{\infty}{\leftrightarrow}$

in

ิำ

$\infty 0^{\circ}$

$125 \mathrm{~Hz}$

Solvent: $\mathrm{CDCl}_{3}$

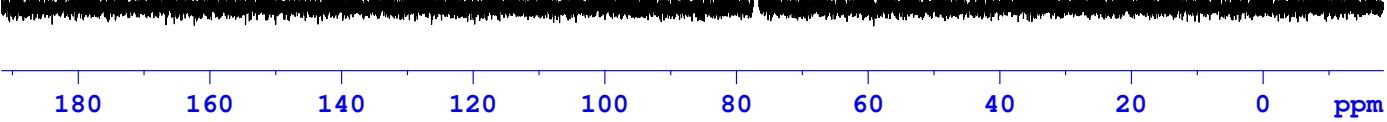



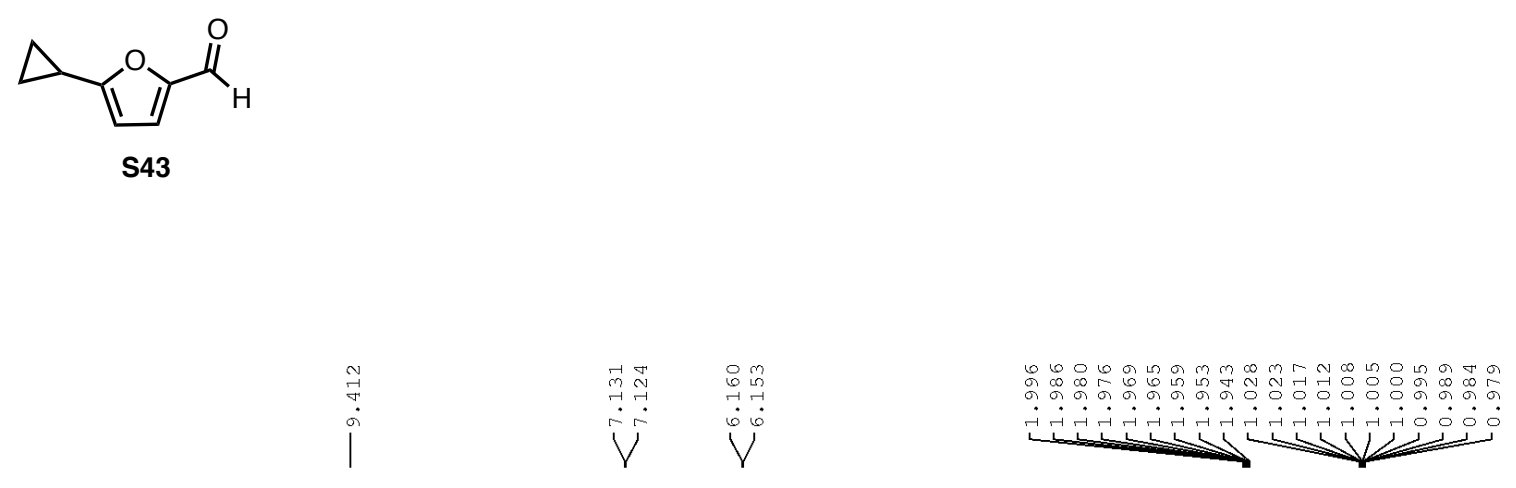

$500 \mathrm{~Hz}$

Solvent: $\mathrm{CDCl}_{3}$

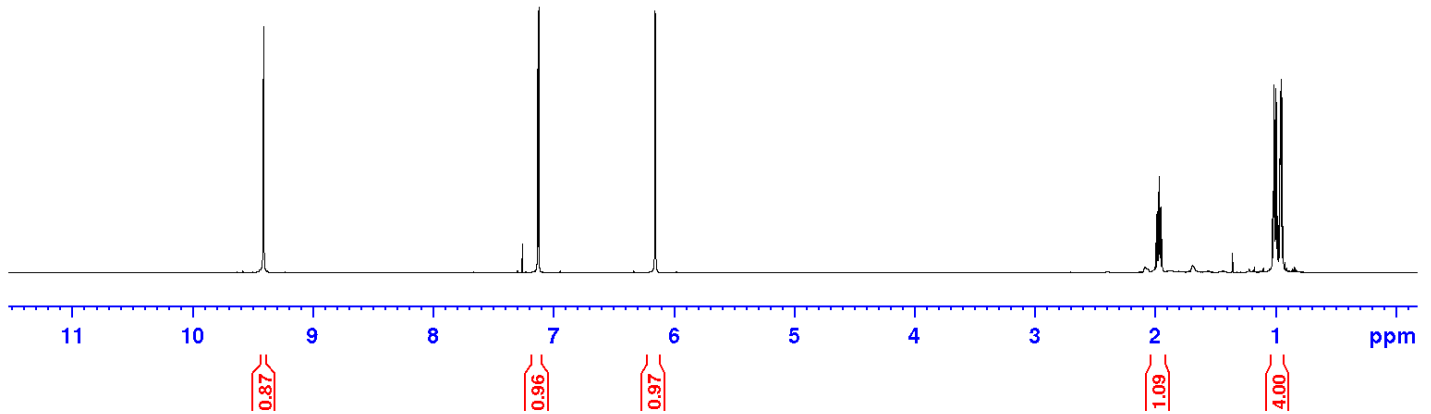



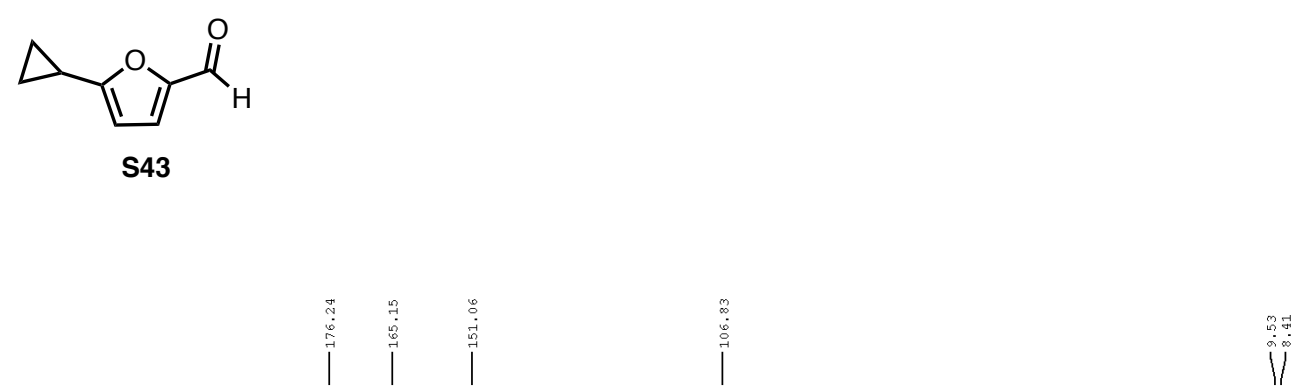

$125 \mathrm{~Hz}$

Solvent: $\mathrm{CDCl}_{3}$

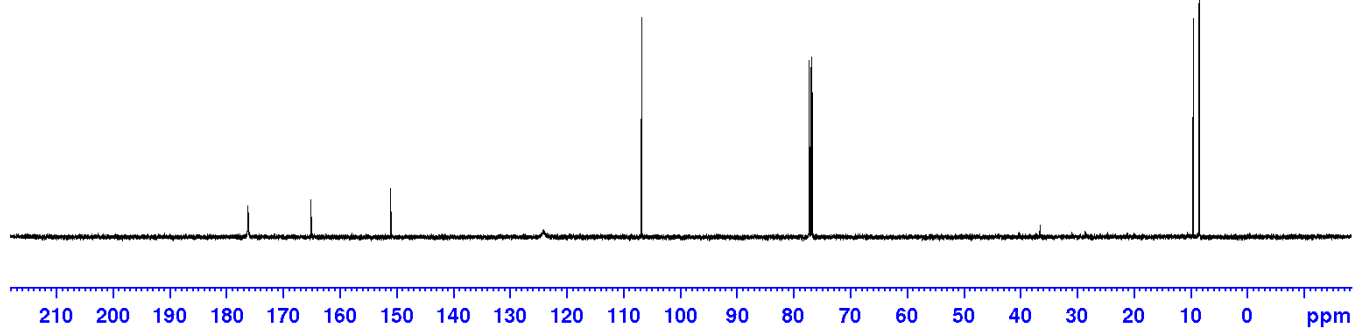

